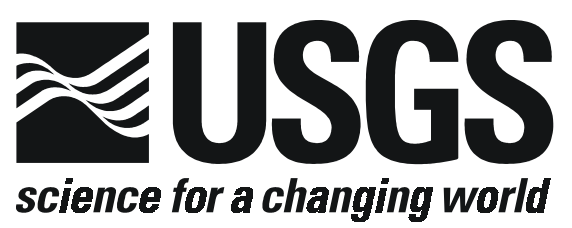

\title{
MAGNETOTELLURIC DATA IN THE MIDDLE RIO GRANDE BASIN, RIO RANCHO, NEW MEXICO
}

\author{
by \\ Jackie M. Williams \\ and \\ Brian D. Rodriguez 1
}

Open-File Report 01-417

on-line edition

2001

This report is preliminary and has not been reviewed for conformity with U.S. Geological Survey editorial standards and stratigraphic nomenclature. Any use of trade, product, or firm names is for descriptive purposes only and does not imply endorsement by the U.S. Government.

U.S. DEPARTMENT OF THE INTERIOR U.S. GEOLOGICAL SURVEY

1 Denver, Colorado 


\section{TABLE OF CONTENTS}

INTRODUCTION

MAGNETOTELLURIC METHOD

MAGNETOTELLURIC SURVEY

MAGNETOTELLURIC DATA

REFERENCES CITED 9

APPENDIX - MAGNETOTELLURIC DATA PLOTS 11 


\section{INTRODUCTION}

The population in the Albuquerque-Santa Fe region of New Mexico is rapidly growing. The Santa Fe Group aquifer in the Middle Rio Grande Basin is the main source of municipal water for the greater Albuquerque metropolitan area. The capacity of this aquifer is more limited than previously thought (Thorn et al., 1993). The Middle Rio Grande Basin, as defined hydrologically and used here, is the area within the Rio Grande Valley extending from Cochiti Dam downstream to the community of San Acacia (Figure 1). Because approximately 600,000 people (40 percent of the population of New Mexico) live in the study area (Bartolino, 1999), water shortfalls could have serious consequences for the state. Future growth and land management in the region depends on accurate assessment and protection of the region's groundwater resources. An important issue in defining the ground water resources is a better understanding of the hydrogeology of the Santa Fe Group and the other sedimentary deposits that fill the Rio Grande rift.

The U.S. Geological Survey (USGS) is currently conducting a series of studies of the Middle Rio Grande Basin in north-central New Mexico to address this issue. One objective of these studies is to improve the hydrogeologic models of the Middle Rio Grande Basin to help land managers plan the development of water supplies. These studies involve a multi-disciplinary approach to better understand the critical aquifers in what is considered to be an intracontinental rift environment. Detailed geologic mapping, high-resolution airborne magnetic surveys, lithologic and geophysical logging of wells, surface-based electrical and magnetic surveys, enhanced satellite imagery, as well as hydrologic and hydrogeochemical data are being used to refine understanding of the aquifer systems in the Middle Rio Grande Basin (Cole et al., 1999). An airborne time domain electromagnetic (TEM) survey was flown for the USGS as part of this work. The primary purpose of the TEM survey was to map changes in electrical resistivity with depth that are related to lithologic variations important to the critical aquifers. However, typical conversion of recorded signal to resistivities obtained from TEM surveys are not unique and depend on proprietary algorithms used by the various companies that collect airborne TEM data. Even though the airborne TEM method has been used for many years as a geophysical prospecting tool, its use in hydrogeological mapping is recent. Therefore, before making maps of resistivity, we first evaluated how deep the TEM inversions were reliable to, based on correlation with well logs and ground electromagnetic surveys (magnetotellurics). This report presents a description of the magnetotelluric (MT) soundings that were used to help calibrate the results of the airborne TEM survey. The purpose of this report is to release the magnetotelluric sounding data; no interpretation of the data is included. 


\section{MAGNETOTELLURIC METHOD}

The magnetotelluric (MT) method is a passive surface geophysical technique, which uses the earth's natural electromagnetic fields to investigate the electrical resistivity structure of the subsurface. The resistivity of geologic units is largely dependent upon their fluid content, porosity, degree of fracturing, temperature, and conductive mineral content (Keller, 1989). Saline fluids within the pore spaces and fracture openings can reduce resistivities in a resistive rock matrix. Also, resistivity can be lowered by the presence of conductive clay minerals, carbon, and metallic mineralization. It is common for altered volcanic rocks to contain authigenic minerals that have resistivities ten times lower than those of the surrounding rocks (Nelson and Anderson, 1992). Increased temperatures cause higher ionic mobility and mineral activation energy, reducing rock resistivities significantly. Unaltered, unfractured igneous rocks are normally very resistive (typically 1,000 ohm-m or greater), whereas fault zones will show low resistivity (less than $100 \mathrm{ohm}-\mathrm{m}$ ) when they are comprised of rocks fractured enough to have hosted fluid transport and consequent mineralogical alteration (Eberhart-Phillips and others, 1995). Carbonate rocks are moderately to highly resistive (hundreds to thousands of ohm-m) depending upon their fluid content, porosity, fracturing, and impurities. Marine shales, mudstones, and clay-rich alluvium are normally very conductive (a few ohm-m to tens of ohm-m). Unaltered, metamorphic rocks (non-graphitic) are moderately to highly resistive (hundreds to thousands of ohm-m). Tables of electrical resistivity for a variety of rocks, minerals and geological environments may be found in Keller (1987) and Palacky (1987).

The MT method can be used to probe the crust from depths of tens of meters to depths of tens of kilometers (Vozoff, 1991). Natural variations of the Earth's magnetic and electric field are measured and recorded at each MT station. The primary frequency bands used by the MT method are 10,000 Hz to $1 \mathrm{~Hz}$ from worldwide lightning activity and $1 \mathrm{~Hz}$ to $0.0001 \mathrm{~Hz}$ from geomagnetic micropulsations. The natural electric and magnetic fields propagate vertically in the earth because the very large resistivity contrast between the air and the earth causes a vertical refraction of both fields transmitted into the earth (Vozoff, 1972).

The natural electric and magnetic fields are recorded in two orthogonal, horizontal directions. The vertical magnetic field ("tipper") is also recorded. The resulting time-series signals are used to derive the tensor apparent resistivities and phases. First, the signals are converted to complex cross-spectra using FFT (fast-Fourier-transform) techniques. Then, least-squares, cross-spectral analysis (Bendat and Piersol, 1971) is used to 
solve for a transfer function that relates the observed electric fields to the magnetic fields under the assumption that the Earth consists of a two-input, two-output, linear system with the magnetic fields as input and the electric fields as output. Prior to conversion to apparent resistivity and phase, the tensor is normally rotated into principal directions that correspond to the direction of maximum and minimum apparent resistivity. For a two-dimensional (2-D) Earth, the MT fields can be de-coupled into transverse electric (TE) and transverse magnetic (TM) modes; 2-D modeling is generally done to fit both modes. When the geology satisfies the 2-D assumption, the MT data for the TE mode is for the electric field parallel to geologic strike, and the data for the TM mode is for the electric field across strike. The MT method is well suited for studying complicated geological environments because the electric and magnetic relations are sensitive to vertical and horizontal variations in resistivity. The method is capable of establishing whether the electromagnetic fields are responding to subsurface terranes of effectively 1-, 2-, or 3-dimensions. An introduction to the MT method and references for a more advanced understanding are contained in Dobrin and Savit (1988) and Vozoff (1991).

\section{MAGNETOTELLURIC SURVEY}

Fourteen MT soundings were acquired in May of 1997 and May of 1998 in the Rio Rancho subdivision of Albuquerque, New Mexico (Figure 1). The station locations were chosen to help calibrate the inversion of the airborne time domain electromagnetic data in areas that had locally 1-D electromagnetic response and for proximity to roads and avoidance of electrical noise, such as power lines. All stations were collected with a portable EMI MT-1 system (EMI, 1996). Horizontal electric fields were sensed using titanium electrodes placed in an L-shaped, three-electrode array with dipole lengths of $30 \mathrm{~m}$. The orthogonal, horizontal magnetic fields in the direction of the electric-field measurement array were sensed using permalloy-cored induction coils. Frequencies sampled ranged from 20,000 to $1 \mathrm{~Hz}$ using single station recordings of both orthogonal horizontal components of the electric and magnetic fields, along with the vertical magnetic field.

The following table shows the fourteen MT station locations as recorded using a global positioning system during field acquisition. Coordinates are referenced to the 1866 Clarke spheroid and North American 1927 Western United States datum. Longitude and latitude format below is decimal degrees. Universal Transverse Mercator units below are in meters. Elevation below is in meters. 


$\begin{array}{clllll}\text { Station } & \text { Longitude } & \text { Latitude } & \text { North }(\mathrm{m}) & \text { East }(\mathrm{m}) & \text { Elev } \\ 1 & -106.92200 & 35.34817 & 3,906,969 & 13,326,034 & 1775 \\ 2 & -106.77783 & 35.34733 & 3,912,809 & 13,338,440 & 1829 \\ 3 & -106.67917 & 35.29967 & 3,907,400 & 13,347,300 & 1700 \\ 4 & -106.49917 & 35.29400 & 3,906,493 & 13,363,686 & 1720 \\ 6 & -106.75667 & 35.17600 & 3,894,901 & 13,339,108 & 1719 \\ 12 & -106.81327 & 35.17281 & 3,893,712 & 13,334,876 & 1781 \\ 10 & -106.84378 & 35.23374 & 3,900,523 & 13,332,222 & 1847 \\ 11 & -106.82655 & 35.23480 & 3,900,611 & 13,333,792 & 1823 \\ 13 & -106.80202 & 35.23152 & 3,900,205 & 13,336,018 & 1804 \\ 14 & -106.78210 & 35.23149 & 3,900,170113,337,831 & 1756 \\ 16 & -106.74807 & 35.23171 & 3,900,13913,340,928 & 1689 \\ 15 & -106.73068 & 35.23316 & 3,900,273 & 13,342,514 & 1701 \\ 5 & -106.68654 & 35.22695 & 3,899,515 & 13,346,519 & 1609 \\ 7 & -106.68118 & 35.22522 & 3,899,314 & 13,347,004 & 1606\end{array}$

\section{MAGNETOTELLURIC DATA}

The recorded time-series data were transformed to the frequency domain and processed to determine a two-dimensional apparent resistivity and phase tensor at each site. Rotation of the impedance tensor to maximum and minimum directions allows for decoupling into the TE and TM modes. Local reference sensors to help reduce bias in the impedance determinations due to instrument or environmental noise (Gamble and others, 1979a; Clarke and others, 1983) were used at all stations. Although true remote reference techniques were not used in our survey, we did sort cross-power files to select optimal signal-to-noise data sets (see Appendix).

The effects of near-surface resistivity anomalies cause "static shifts" (Sternberg et al., 1988) in the data. Static shifts are significant for half of this data set. Rio Rancho stations 5, 7, 10,11, 13, 14 and 16 had a static shift larger than one-third of a log decade. The remainder of the stations had minor static shifts, ranging from 0.0 to less than 0.3 of a log decade. Cultural features can affect the response of the MT system. Fences, pipelines, communication lines, railways and other man-made conductors can contaminate the responses. Cultural noise appeared to severely affect Rio Rancho stations 5, 6 , and 7 .

The figures in the Appendix represent the field-processed MT data for each station after the time series data were converted to the frequency domain and the tensor-transfer function was rotated into principal directions as described above in the "Magnetotelluric Method" section. 
For each station, nine separate plots are given:

1. Apparent Resistivity for the rotated maximum (x symbol) and minimum (o symbol) modes

2. Impedance Phase for the rotated maximum ( $x$ symbol) and minimum (o symbol) modes

3. Rotation Angle for the impedance tensor (corresponds to the direction of maximum apparent resistivity)

4. Impedance Skew for the impedance tensor

5. Multiple Coherency for the rotated maximum ( $x$ symbol) and minimum (o symbol) modes of the electric field

6. Impedance Polar Plots (at 12 selected frequencies)

7. Tipper Magnitude for the vertical magnetic field

8. Tipper Strike for the vertical magnetic field, and

9. $\mathrm{HzHx}$ (x symbol) and HzHy (o symbol) Coherency

Error bars on the Apparent Resistivity, Impedance Phase, Skew, Tipper Magnitude, and Tipper Strike plots represent probable errors within one standard deviation of the sample variance (Gamble and others, 1979b).

Apparent resistivity is a measure of the magnitude of the electric field strength over the magnetic field strength for a given frequency. The impedance phase is proportional to the slope of the apparent resistivity curve on a log-log plot, but from a baseline at -45 degrees (Vozoff, 1991). A measure of the dimensionality for MT data is provided by the impedance skew of the impedance tensor (Vozoff, 1972). If the effective measured resistivity response to the geology beneath a MT station is truly 1-D or 2-D, then the skew will be zero. Both instrument and environmental sources of noise contribute to non-zero skew values, but are typically small (about 0.1) for relatively low noise level recordings. Higher skews (above 0.2) are an indication of either the resistivity response to 3-D geology or higher levels of noise. Man-made electrical noise, such as power lines, power generators, moving vehicles and trains can have a negative effect on MT data quality. All these local disturbances produce an incoherent noise mainly affecting frequencies above 1

$\mathrm{Hz}$. Other man-made electrical noise, such as direct current electric trains and active cathodic protection of pipelines produce coherent electromagnetic signals mainly affecting frequencies below $1 \mathrm{~Hz}$.

In the survey area, noise from a number of small power lines and small moving vehicles was negligible at distances of $0.4 \mathrm{~km}$ and greater from the noise source. Power line levels were measured at each site and were typically less than 20\% of the maximum recordable signals. Noise from larger power lines, power generators, pipelines, and trains was negligible at least $5 \mathrm{~km}$ from them. Recordings were not made when noise from moving vehicles affected the magnetic signals. Local lightning, wind, 
and rainstorms can also degrade data quality, but these were avoided by not recording during active thunderstorm periods. Wind noise was minimized by burying the magnetic induction coils.

Predicted values of the electric field can be computed from the measured values of the magnetic field (Vozoff, 1991). The coherence of the predicted electric field with the measured electric field is a measure of the signal-to-noise ratio provided in the multiple coherency plot. Values are normalized between 0 and 1 , where values at 0.5 signify signal levels equal to noise levels. For this data set, coherencies were generally at an acceptable level, except at times in the high frequency "dead band" $(1,000$ to $5,000 \mathrm{~Hz})$.

The figures in the Appendix represent the field-processed MT data at each station, which includes some data scatter and poor signal-to-noise ratios. Our only effort at removing noisy data points was to visually inspect and select the best signal-tonoise field data to combine into the final data plots.

The impedance polar plots provide a measure of the MT data dimensionality (Reddy and others, 1977). For 1-D resistivity structures, the principal impedance polar diagram (dashed line) is a circle. For 2-D or 3-D resistivity structures, the principal impedance polar diagram (dashed line) elongates either parallel or perpendicular to strike direction. Over resistors, the principal impedance polar diagram elongates perpendicular to strike direction and over conductors, the principal impedance polar diagram elongates parallel to strike direction. Also, for 2-D resistivity structures, the additional impedance polar diagram (solid line) attains the shape of a symmetric clover leaf. For 3-D resistivity structures, the additional impedance polar diagram (solid line) elongates in one direction and its amplitude is comparable to that of the principal impedance polar diagram (dashed line). The only site whose polar plots indicated 3-D character was Rio Rancho station 7.

The tipper can be calculated when the vertical component of the magnetic field is measured. The tipper magnitude is a measure of the tipping of the magnetic field out of the horizontal plane (Vozoff, 1991). The magnitude is zero for the $1-D$ case and typically increases between 0.1 to 0.5 , and rarely as great as 1 , as it responds to vertical and sub-vertical structures. The tipper strike is typically used to help resolve the 90-degree ambiguity in the impedance rotation angle. The vertical component of the magnetic field was measured at all stations. The tipper magnitude of these stations was typically 0.1 to 1.2 over the lower frequencies indicating vertical structure at depth. The $\mathrm{HzHx}$ and $\mathrm{HzHy}$ coherency is a measure of the signal-to-noise ratio of the vertical magnetic field with respect to each of the orthogonal horizontal magnetic field directions. Values are normalized between 0 and 1, where values at 0.5 signify signal levels equal to noise levels. These threecomponent magnetic field coherencies provide a check on the 
signal-to-noise ratio of the measured values in the tipper magnitude and tipper strike plots.

Acknowledgments. We thank Jay A. Sampson of the U.S. Geological Survey for his data acquisition contributions to this study.

\section{REFERENCES CITED}

Bendat, J.S., and Piersol, A.G., 1971, Random Data: Analysis and Measurement Procedures: New York, Wiley Interscience, $407 p$.

Clarke, J., Gamble, T.D., Goubau, W.M., Koch, R.H., and Miracky, R.F., 1983, Remote-reference magnetotellurics: Equipment and procedures: Geophysical Prospecting, vol. 31, p. 149-170.

Dobrin, M.D., and Savit, C.H., 1988, Introduction to Geophysical Prospecting (4th ed.): New York, McGraw-Hill, $867 p$.

Eberhart-Phillips, D., Stanley, W.D., Rodriguez, B.D. and Lutter, W.J., 1995, Surface seismic and electrical methods to detect fluids related to faulting: Journal of Geophysical Research, vol. 100, no. B7, p. 12,919-12,936.

EMI, 1996, MT-1 magnetotelluric system operation manual, version 3.2: ElectroMagnetic Instruments, Inc., Richmond, California, $220 \mathrm{p}$.

Gamble, T.D., Goubau, W.M. and Clarke, J., 1979a, Magnetotellurics with a remote magnetic reference: Geophysics, v. 44, no. 1, p. 53-68.

Gamble, T.D., Goubau, W.M. and Clarke, J., 1979b, Error analysis for remote reference magnetotellurics: Geophysics, v. 44, no. 5, p. 959-968.

Keller, G.V., 1987, Rock and mineral properties, in Electromagnetic Methods in Applied Geophysics Theory: M.N. Nabighian, Ed., Society of Exploration Geophysicists, Tulsa, Oklahoma, v. 1, p. 1351 .

Keller, G.V., 1989, Electrical properties, in Carmichael, R.S., Ed., Practical handbook of physical properties of rocks and minerals: CRC Press, Boca Raton, Florida, p. 359-427.

Nelson, P.H. and Anderson, L.A., 1992, Physical properties of ash flow tuff from Yucca Mountain, Nevada: Journal of Geophysical Research, vol. 97, no. B5, p. 6823-6841. 
Palacky, G.J., 1987, Resistivity characteristics of geologic targets, in Electromagnetic Methods in Applied Geophysics Theory: M.N. Nabighian, Ed., Society of Exploration Geophysicists, Tulsa, Oklahoma, vol. 1, p. 53129.

Reddy, I.K., Rankin, D., and Phillips, R.J., 1977, Threedimensional modeling in magnetotelluric and magnetic variational sounding: Geophysics Journal of the Royal Astronomical Society, vol. 51, p. 313-325.

Sternberg, B.K., Washburne, J.C., and Pellerin, L., 1988, Correction for the static shift in magnetotellurics using transient electromagnetic soundings: Geophysics, vol. 53, p. 1459-1468.

Thorn, C.R., McAda, D.P., and Kernodle, J.M., 1993, Geohydrologic framework and hydrologic conditions in the Albuquerque Basin, central New Mexico: U.S. Geological Survey WaterResources Investigations Report 93-4149, 106 p., 2 plates.

Vozoff, K., 1972, The magnetotelluric method in the exploration of sedimentary basins: Geophysics, vol. 37, p. 98-141.

Vozoff, K., 1991, The magnetotelluric method, in Electromagnetic methods in applied geophysics: M.N. Nabighian, Ed., Society of Exploration Geophysicists, Tulsa, Oklahoma, vol. 2, part B, p. 641-711. 


\section{APPENDIX MAGNETOTELLURIC DATA PLOTS}

There are nine separate plots for each station:

1. Apparent Resistivity for the rotated maximum ( $x$ symbol) and minimum (o symbol) modes

2. Impedance Phase for the rotated maximum ( $x$ symbol) and minimum (o symbol) modes

3. Rotation Angle for the impedance tensor (corresponds to the direction of maximum apparent resistivity)

4. Impedance Skew for the impedance tensor

5. Multiple Coherency for the rotated maximum ( $x$ symbol) and minimum (o symbol) modes of the electric field

6. Impedance Polar Plots (at 12 selected frequencies)

7. Tipper Magnitude for the vertical magnetic field

8. Tipper Strike for the vertical magnetic field, and

9. HzHx (x symbol) and HzHy (o symbol) Coherency

Refer to the "Magnetotelluric Data" section in this report for an explanation of these plots. 


\section{Station 1}

Rio Rancho - Albuquerque, NM

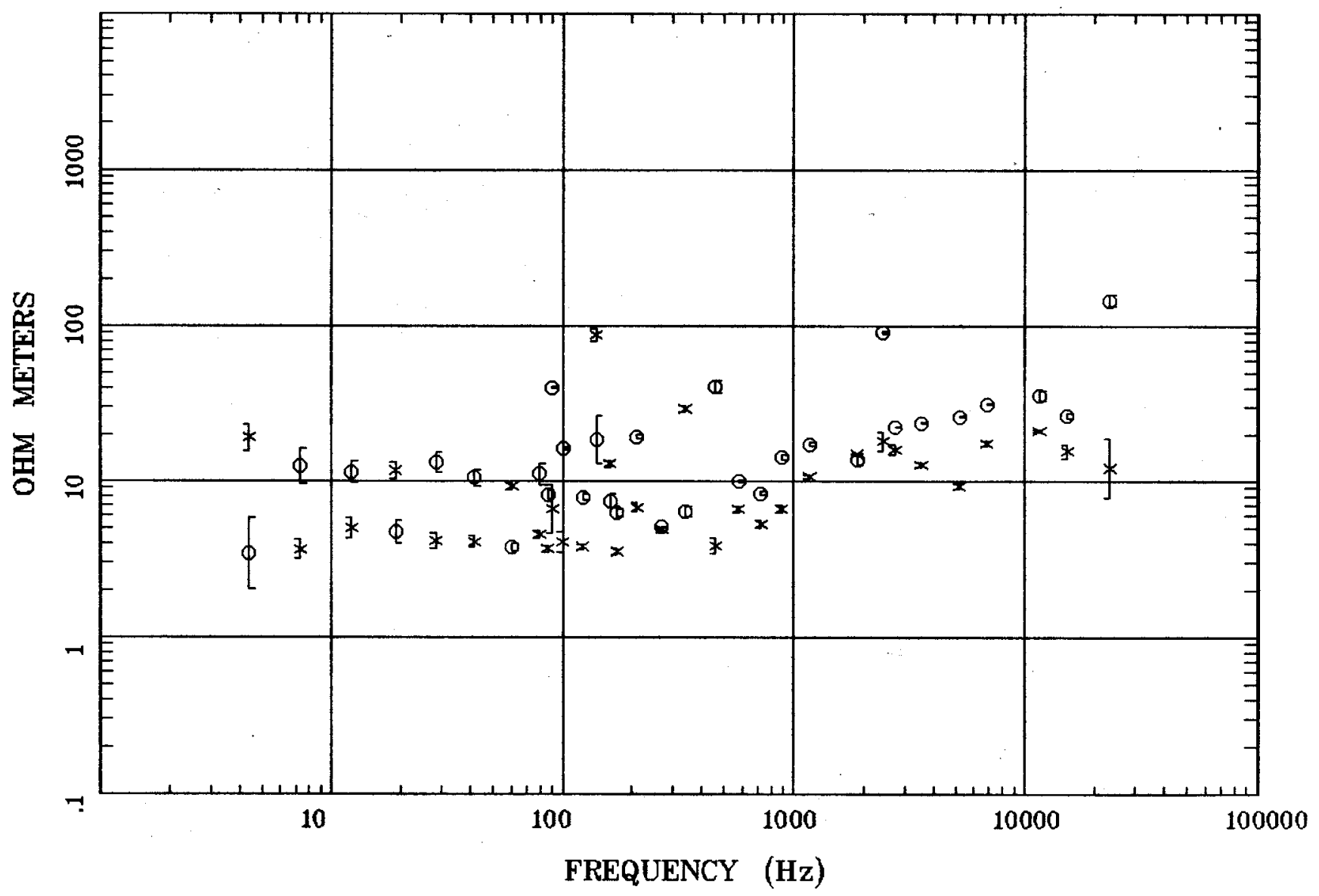

Client:

Remote: Local E

Acquired: 14:5 May 23, 1997

Survey Co:USGS GD-MRP Denver
Rotation:

Filename: rro1.all

Channels: Ch1 Ch2 Ch3 Ch4 Ch5 Ch6 Ch7

Plotted: 15:15 Mar 08, 2001

$<$ EMI - ElectroMagnetic Instruments 
Rio Rancho - Albuquerque, NM

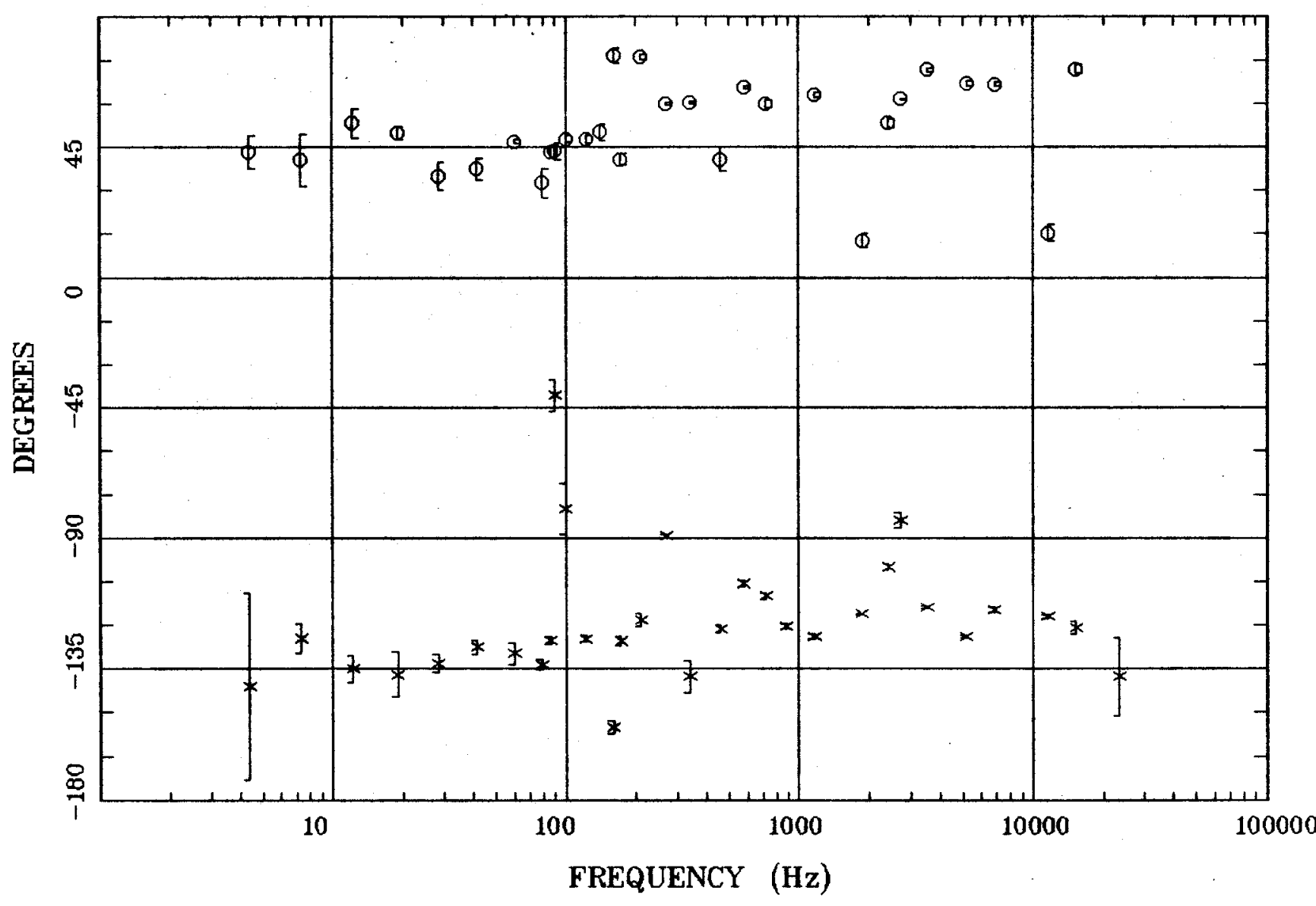

Client:

Remote: Local E

Acquired: 14:5 May 23, 1997 Survey Co:USGS GD-MRP Denver
Rotation:

Filename: rro1.all

Channels: Ch1 Ch2 Ch3 Ch4 Ch5 Ch6 Ch7

Plotted: 15:15 Mar 08, 2001

< EMI - ElectroMagnetic Instruments 


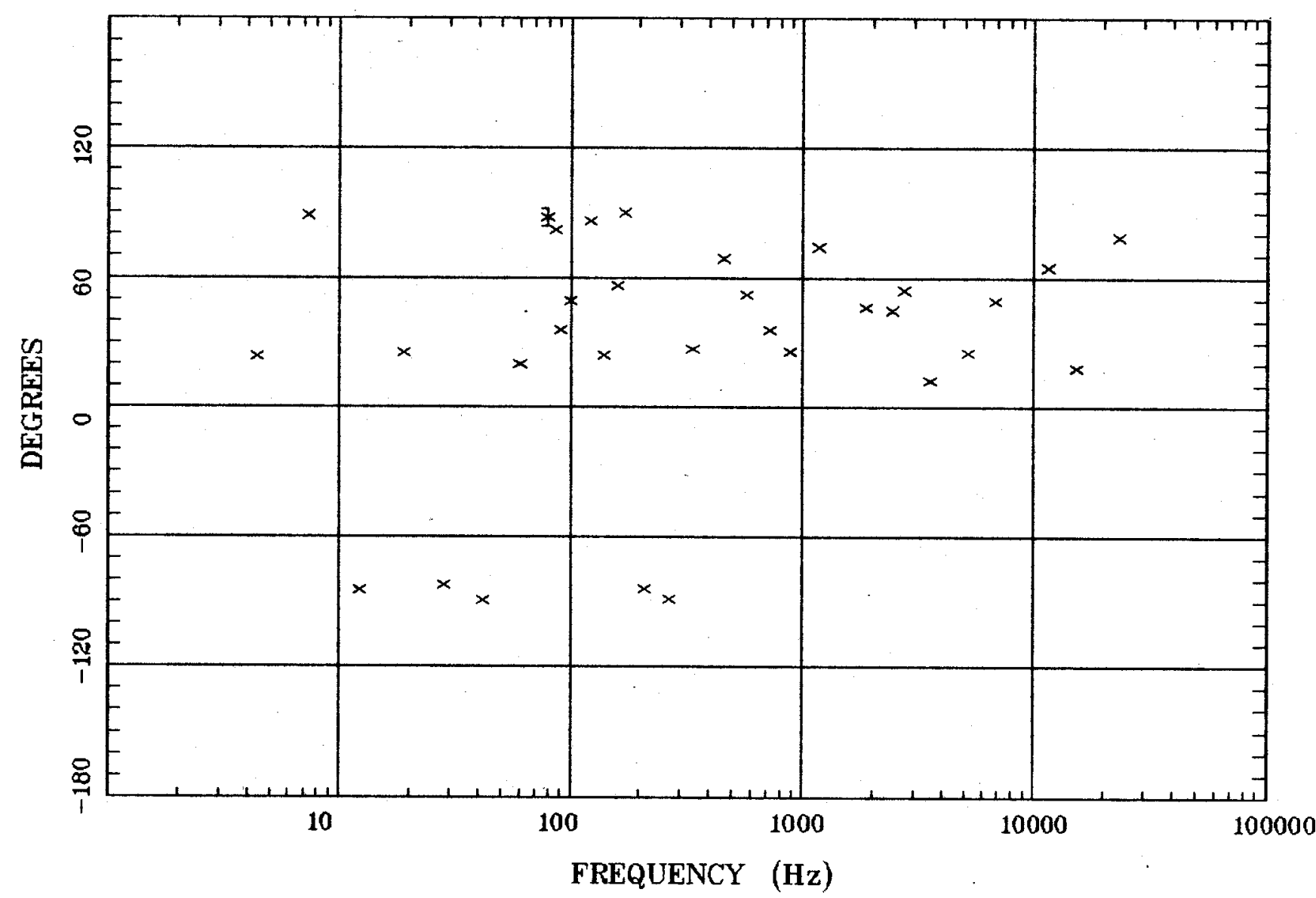

Client:

Remote: Local E

Acquired: 14:5 May 23, 1997 Survey Co:USGS GD-MRP Denver
Rotation:

Filename: rro1.all

Channels: Ch1 Ch2 Ch3 Ch4 Ch5 Ch6 Ch7

Plotted: 15:16 Mar 08, 2001

$<$ EMI - ElectroMagnetic Instruments > 


\section{Station 1}

IMPEDANCE SKEW

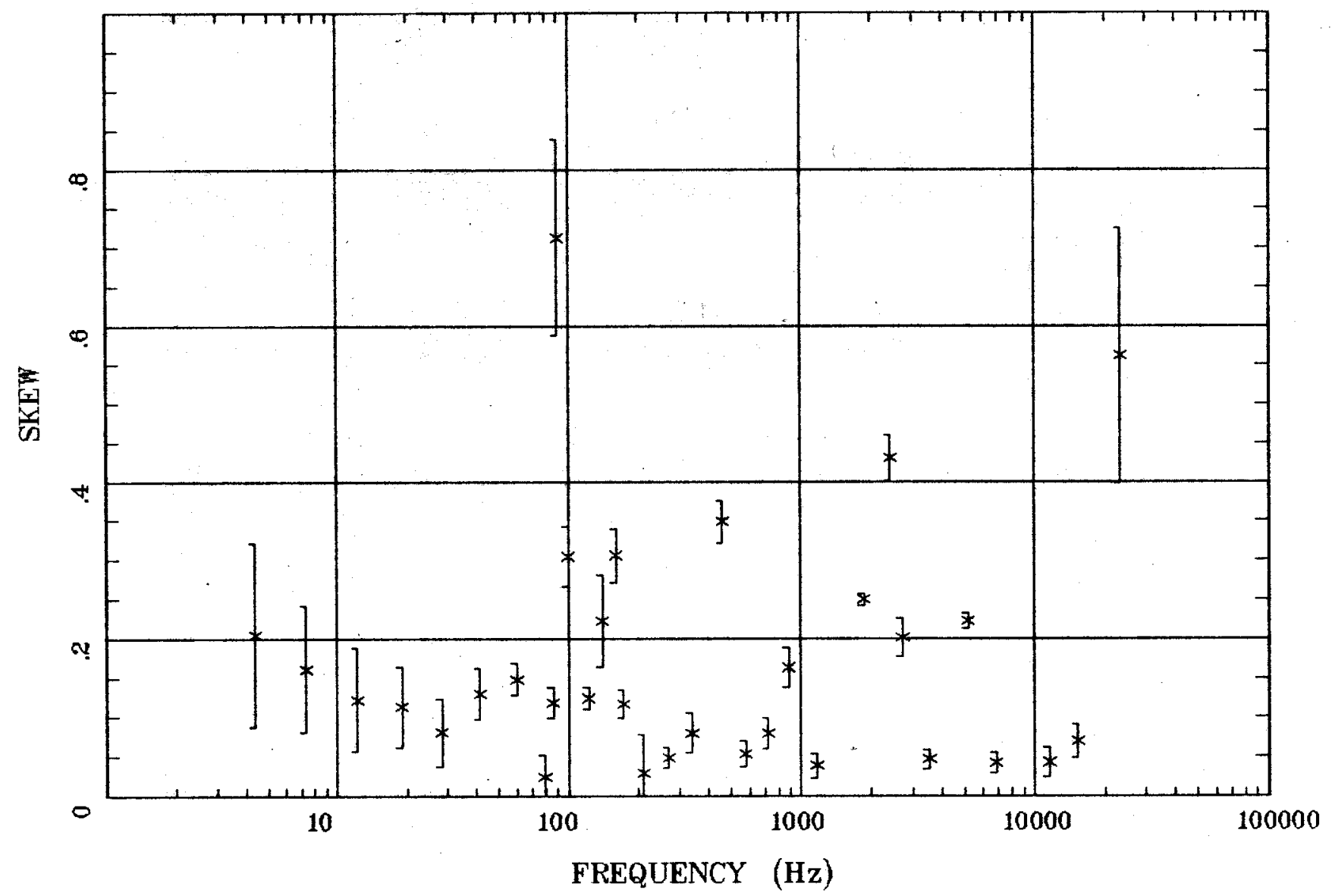

Rio Rancho - Albuquerque, NM

Rotation:

Filename: rr01.all

Channels: Ch1 Ch2 Ch3 Ch4 Ch5 Ch6 Ch7

Plotted: 15:16 Mar 08, 2001

$<$ EMI - ElectroMagnetic Instruments
Client:

Remote: Local $\mathrm{E}$

Acquired: 14:5 May 23, 1997 Survey Co:USGS GD-MRP Denver 


\section{Station 1}

E MULT Coh.

Rio Rancho - Albuquerque, NM

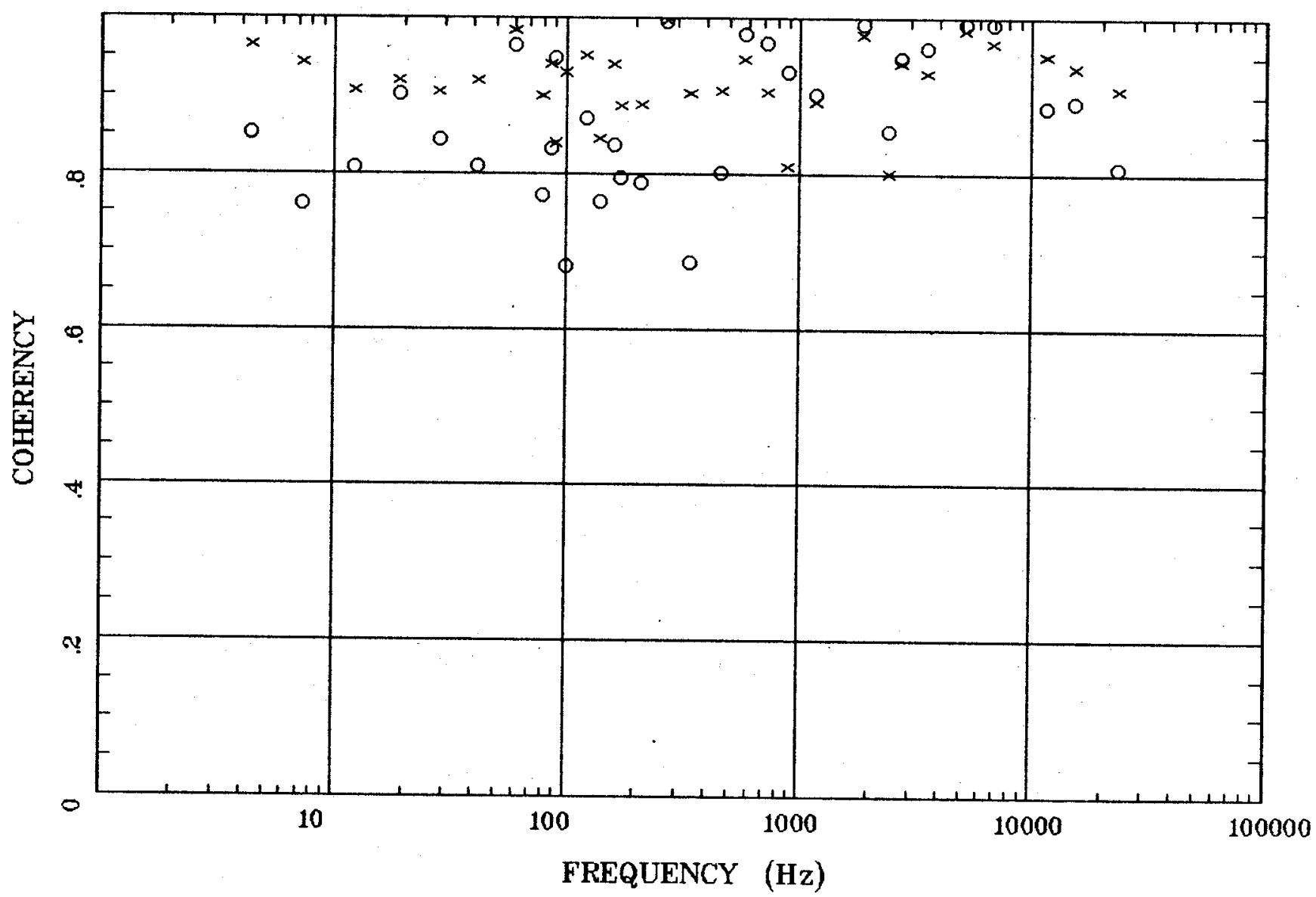

Client:

Remote: Local E

Acquired: 14:5 May 23, 1997 Survey Co:USGS GD-MRP Denver
Ratation:

Filename: rro1.all

Channels: Ch1 Ch2 Ch3 Ch4 Ch5 Ch6 Ch7

Plotted: 15:16 Mar 08, 2001

< EMI - ElectroMagnetic Instruments > 


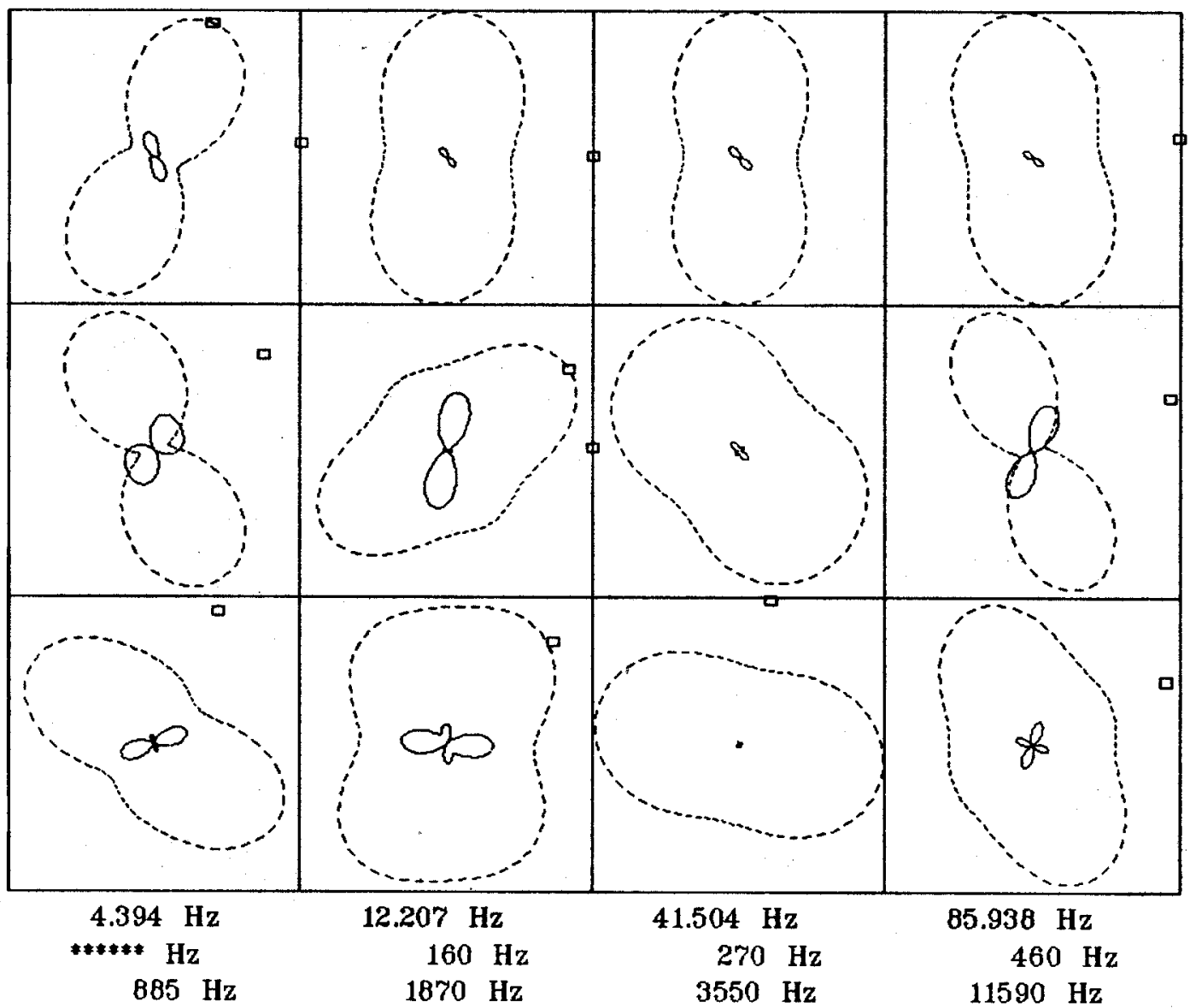

Client:

Remote: Local E

Acquired: 14:5 May 23, 1997

Survey Co:USGS GD-MRP Denver
Rotation:

Filename: rro1.all

Channels: Ch1 Ch2 Ch3 Ch4 Ch5 Ch6 Ch7

plotted: 15:16 Mar 08, 2001

< EMI - ElectroMagnetic Instruments 


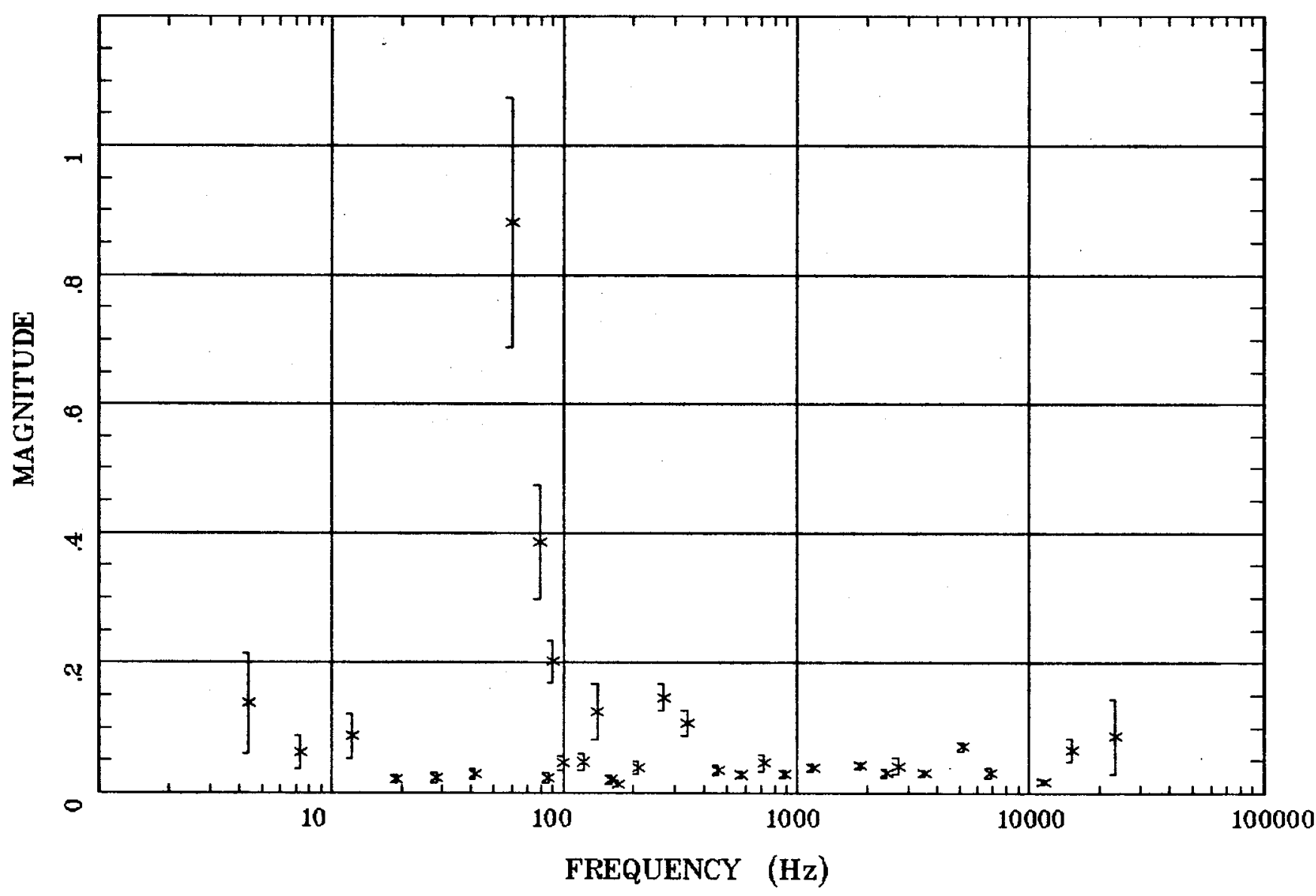

Client:

Remote: Local E Acquired: 14:5 May 23, 1997 Survey Co:USGS GD-MRP Denver
Rotation:

Filename: rro1.all

Channels: Ch1 Ch2 Ch3 Ch4 Ch5 Ch6 Ch7

Plotted: 15:16 Mar 08, 2001

< EMI - ElectroMagnetic Instruments > 
TIPPER STRIKE

Rio Rancho - Albuquerque, NM

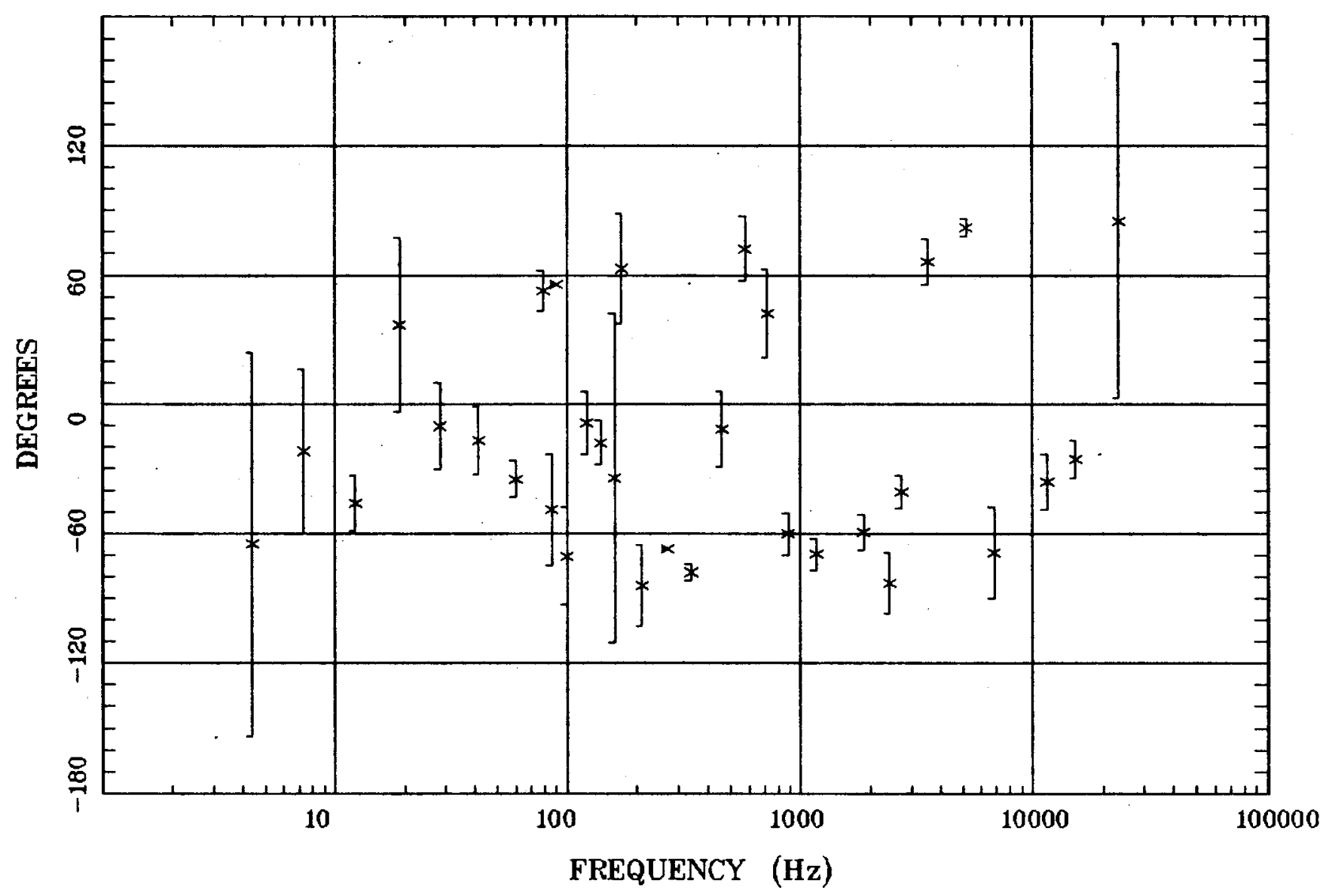

Client:

Remote: Local E

Acquired: 14:5 May 23, 1997

Survey Co:USGS GD-MRP Denver
Rotation:

Filename: rro1.all

Channels: Ch1 Ch2 Ch3 Ch4 Ch5 Ch6 Ch7

Plotted: 15:16 Mar 08, 2001

< EMI - ElectroMagnetic Instruments > 
Rio Rancho - Albuquerque, NM

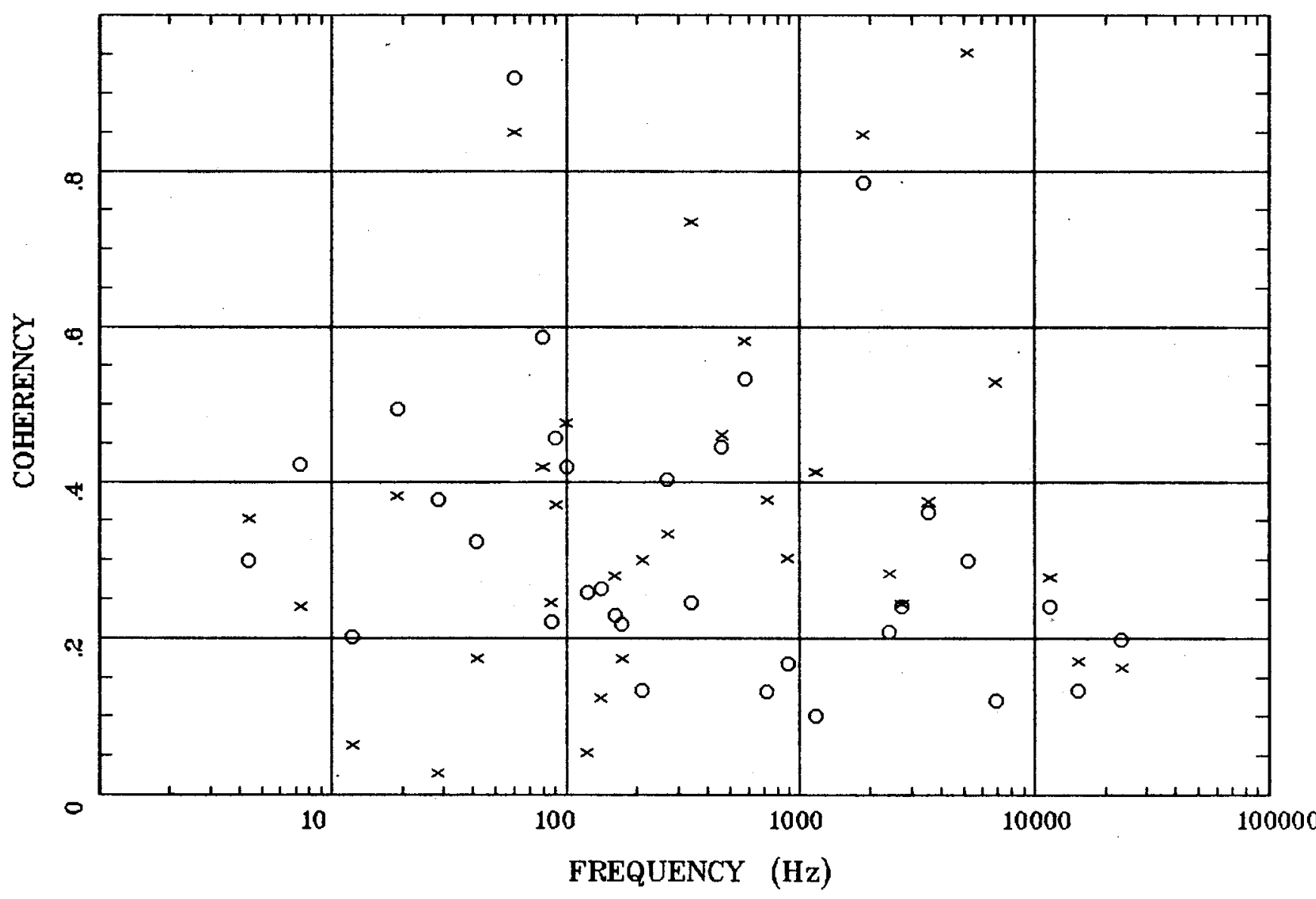

Client:

Remote: Local E

Acquired: 14:5 May 23, 1997 Survey Co:USGS GD-MRP Denver
Rotation:

Filename: rro1.all

Channels: Ch1 Ch2 Ch3 Ch4 Ch5 Ch6 Ch7

Plotted: 15:16 Mar 08, 2001

< EMI - ElectroMagnetic Instruments > 


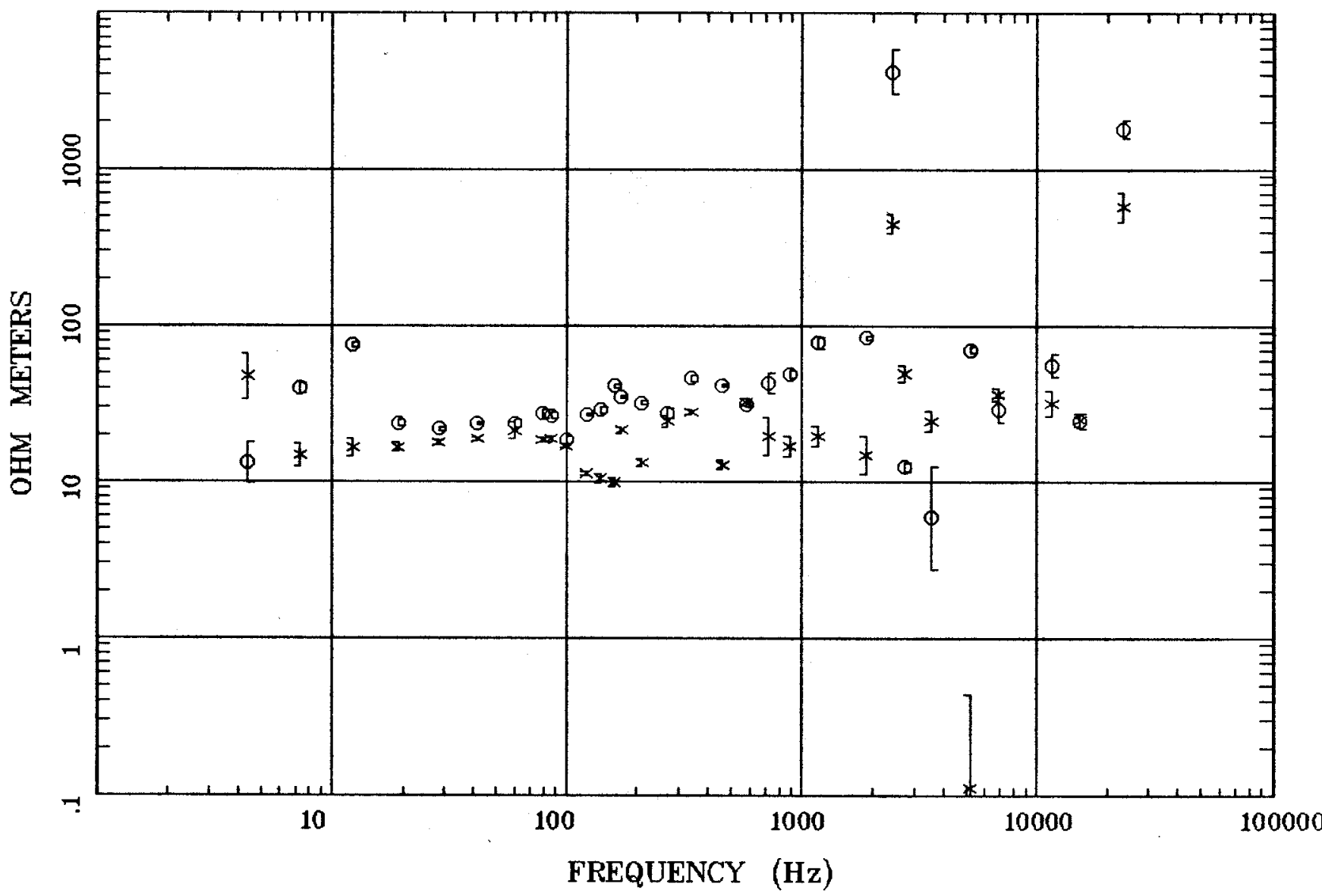

Client:

Remote: Local E Acquired: 10:3 May 23, 1997 Survey Co:USGS GD-MRP Denver
Rotation:

Filename: rro2a1.all

Channels: Ch1 Ch2 Ch3 Ch4 Ch5 Ch6 Ch7

Plotted: 15:16 Mar 08, 2001

$<$ EMI - ElectroMagnetic Instruments 
Station 2

IMPEDANCE PHASE

Rio Rancho - Albuquerque, NM

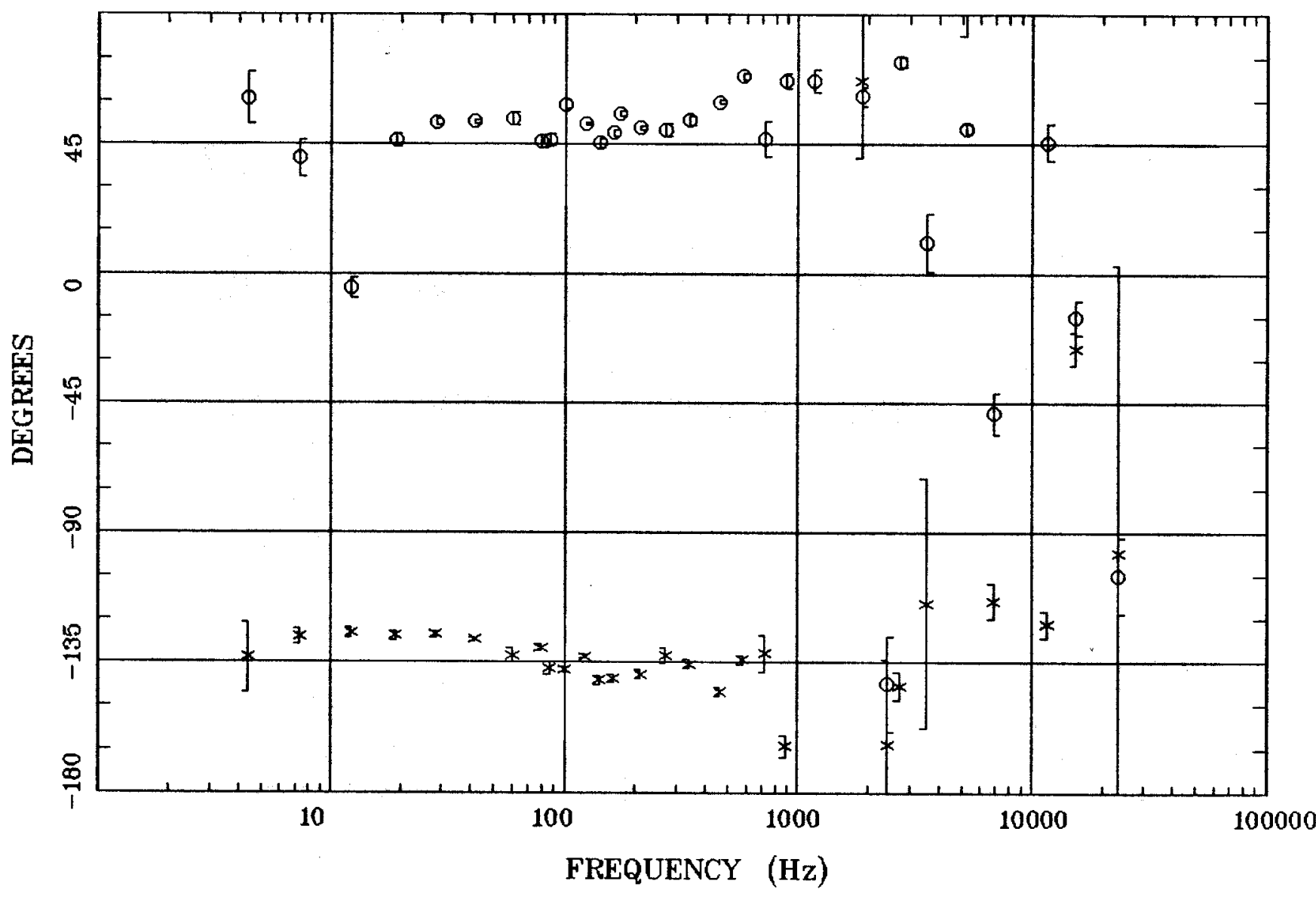

Client:

Remote: Local E

Acquired: 10:3 May 23, 1997

Survey Co:USGS GD-MRP Denver
Rotation:

Filename: rro2a1.all

Channels: Ch1 Ch2 Ch3 Ch4 Ch5 Ch6 Ch7

Plotted: 15:16 Mar 08, 2001

< EMI - ElectroMagnetic Instruments 
Station 2

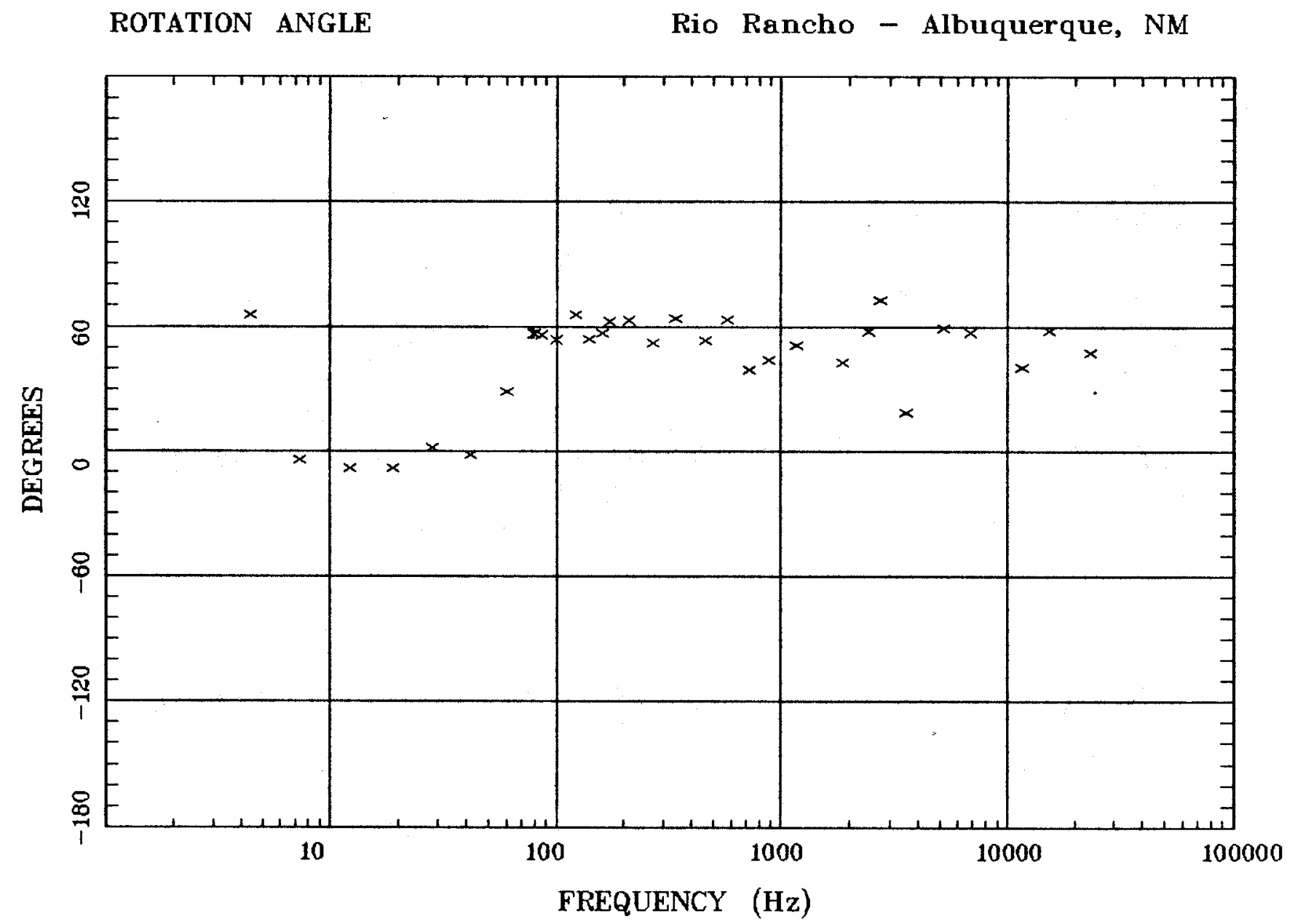

Client:

Rotation:

Remote: Local E

Acquired: 10:3 May 23, 1997

Filename: rro2a1.all

Channels: Ch1 Ch2 Ch3 Ch4 Ch5 Ch6 Ch7

Plotted: 15:16 Mar 08, 2001

Survey Co:USGS GD-MRP Denver

< EMI - ElectroMagnetic Instruments 
IMPEDANCE SKEW

Rio Rancho - Albuquerque, NM

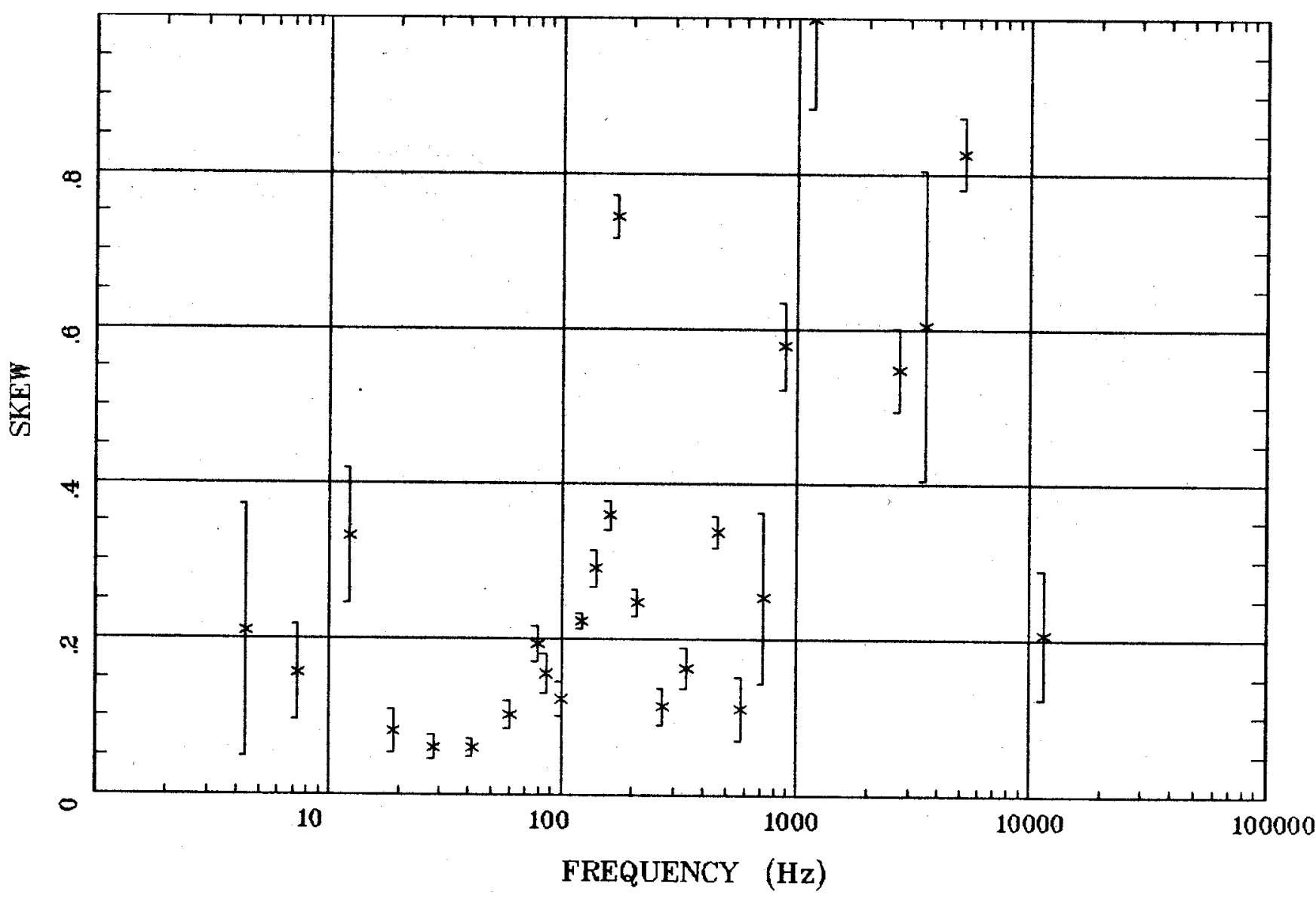

Client:

Remote: Lacal E

Acquired: 10:3 May 23, 1997 Survey Co:USGS GD-MRP Denver
Rotation:

Filename: rroza1.all

Channels: Ch1 Ch2 Ch3 Ch4 Ch5 Ch6 Ch7

Plotted: 15:16 Mar 08, 2001

<EMI - ElectroMagnetic Instruments > 


\section{Station 2}

E MULT Coh.

Rio Rancho - Albuquerque, NM

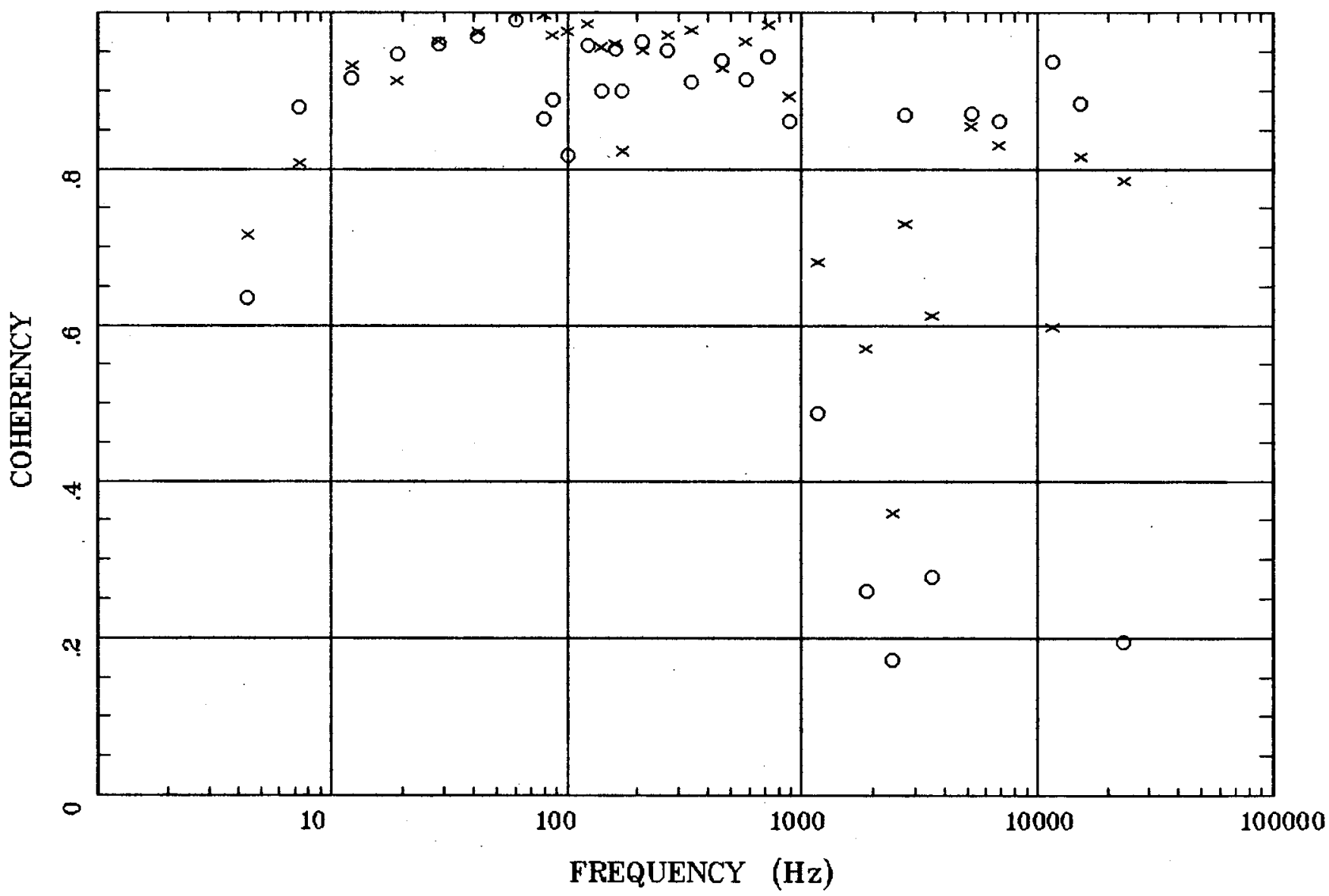

Client:

Remote: Local E

Acquired: 10:3 May 23, 1997

Survey Co:USGS GD-MRP Denver
Rotation:

Filename: rro2a1.all

Channels: Ch1 Ch2 Ch3 Ch4 Ch5 Ch6 Ch7

Plotted: 15:16 Mar 08, 2001

\& EMI - ElectroMagnetic Instruments > 


\section{POLAR PLOTS}

Rio Rancho - Albuquerque, NM

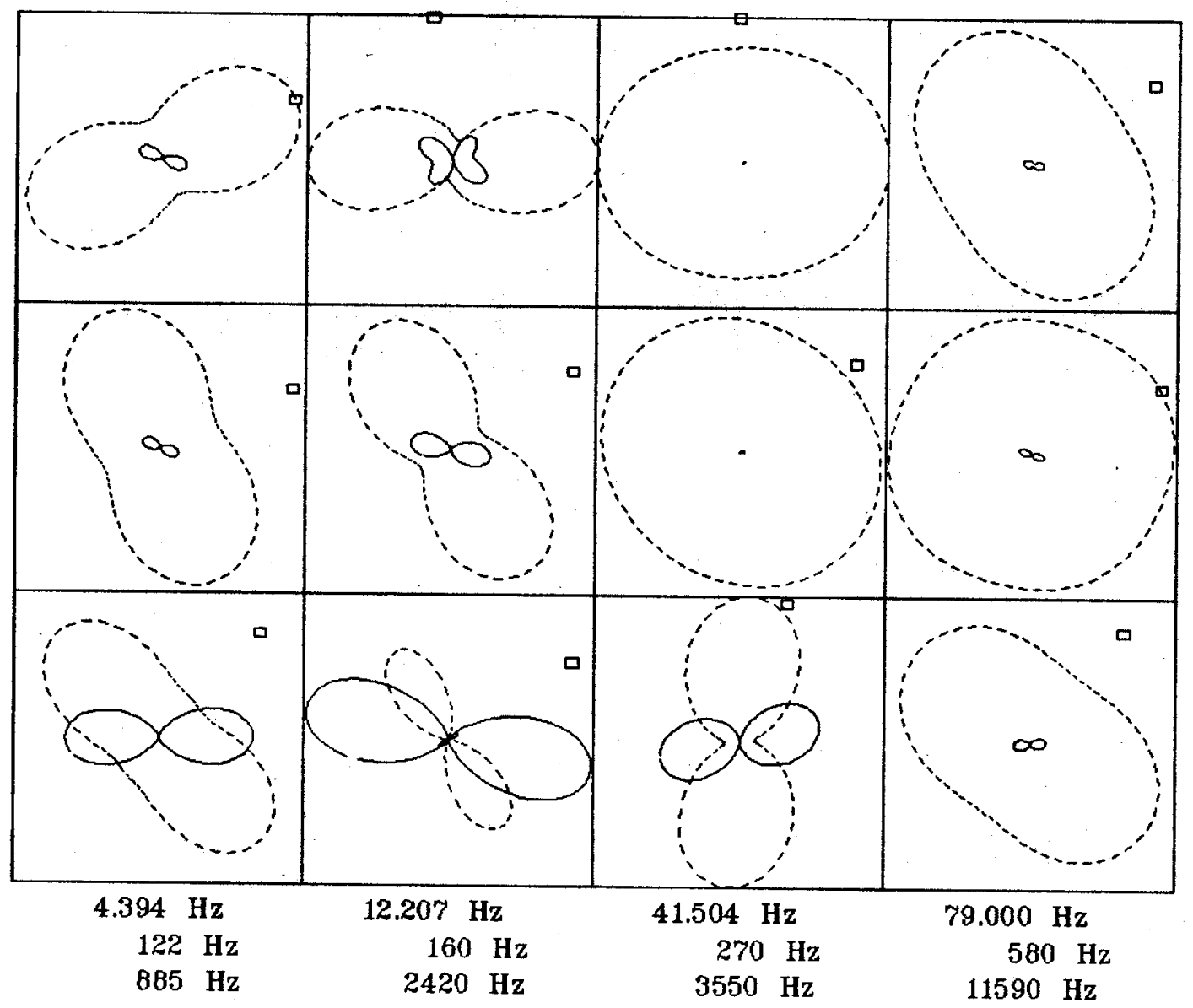

Client:

Remote: Local E

Acquired: 10:3 May 23, 1997 Survey Co:USGS GD-MRP Denver
Rotation:

Filename: rr02a1.all

Channels: Ch1 Ch2 Ch3 Ch4 Ch5 Ch6 Ch7 Plotted: 15:16 Mar 08, 2001

< EMI - ElectroMagnetic Instruments > 
TIPPER MAGNITUDE

Rio Rancho - Albuquerque, NM

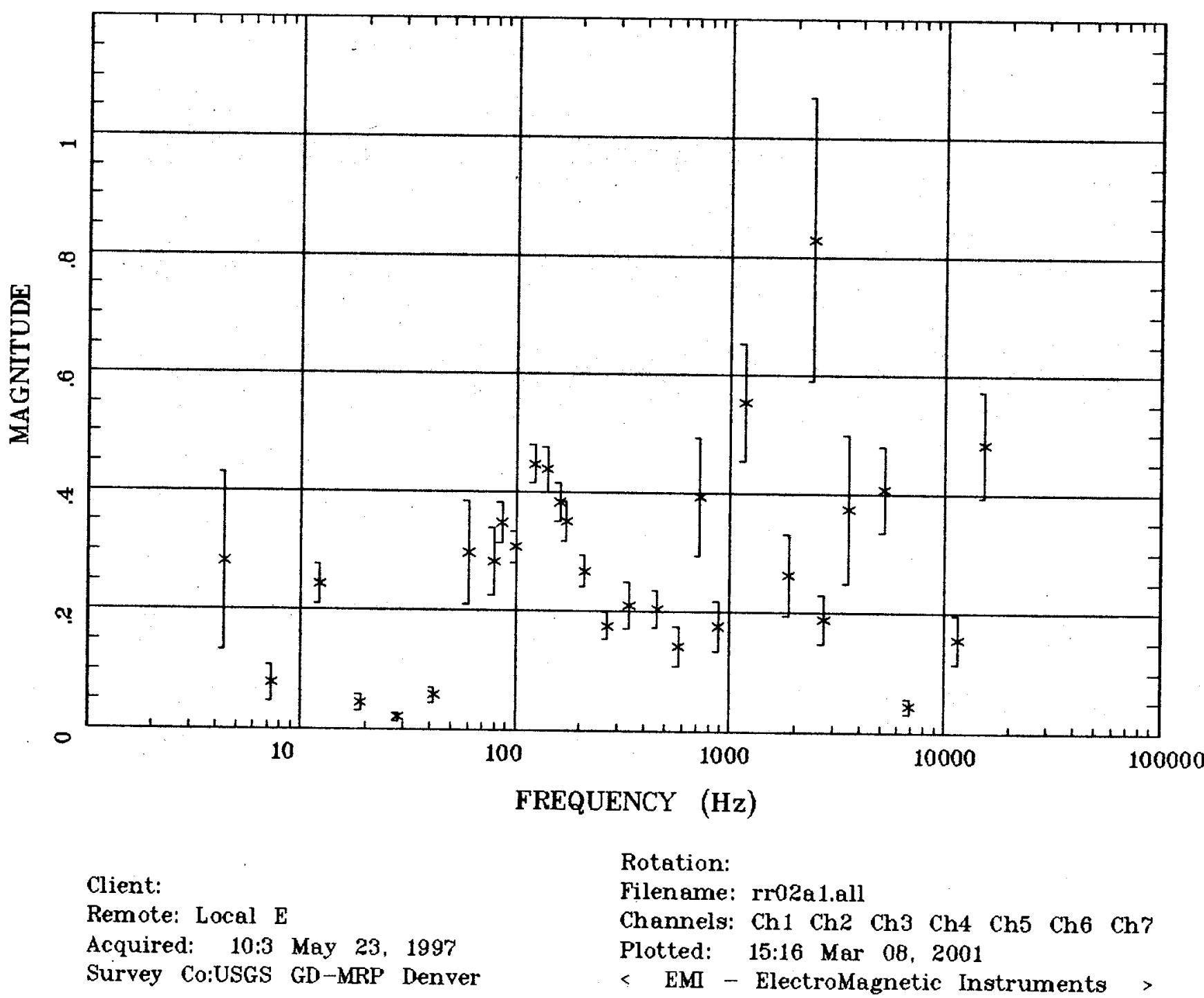


Station 2

TIPPER STRIKE

Rio Rancho - Albuquerque, NM

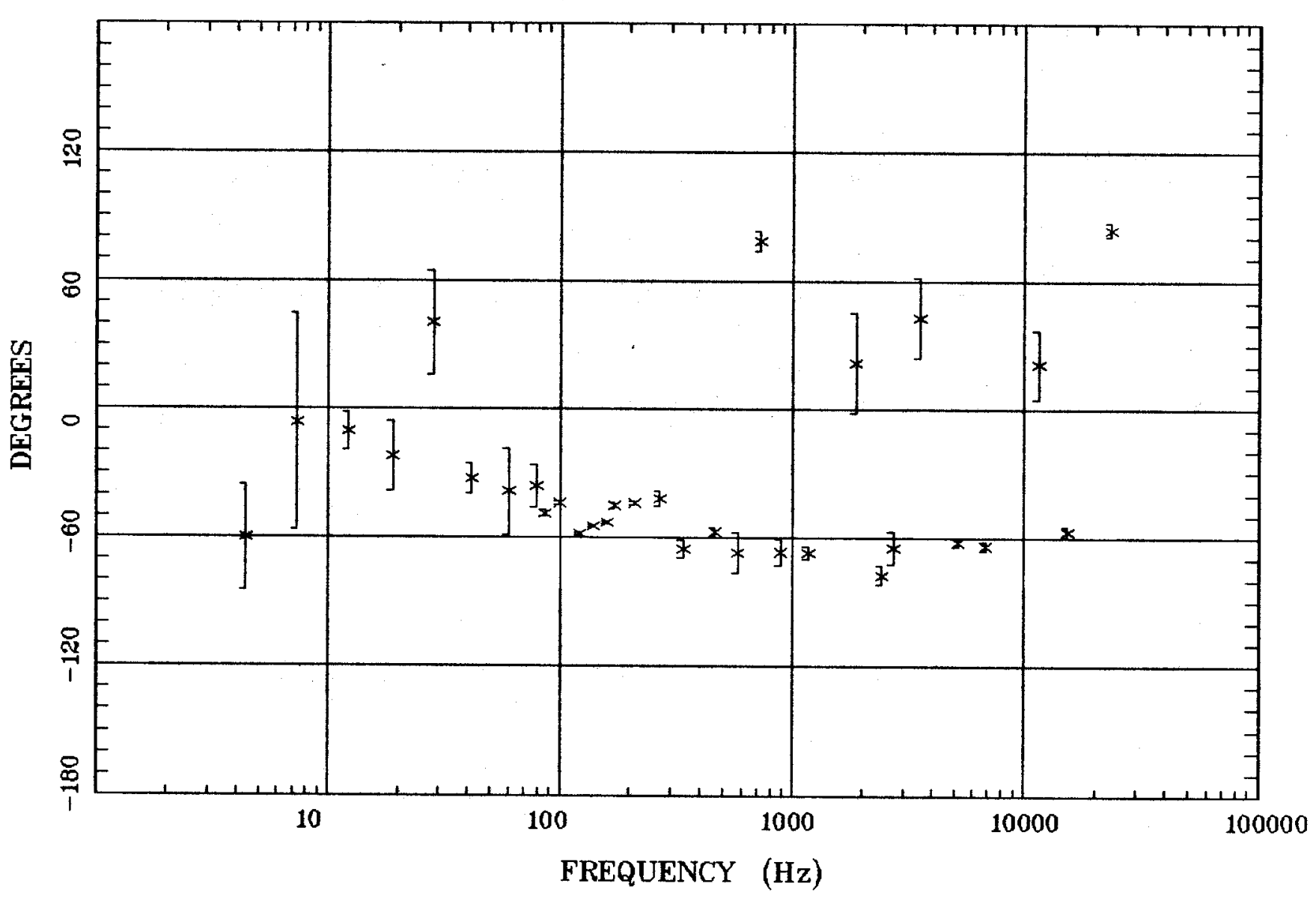

Client:

Remote: Local E

Acquired: 10:3 May 23, 1997

Survey Co:USGS GD-MRP Denver
Rotation:

Filename: rro2a1.all

Channels: Ch1 Ch2 Ch3 Ch4 Ch5 Ch6 Ch7

Plotted: 15:16 Mar 08, 2001

< EMI - ElectroMagnetic Instruments 
Station 2

HzHx.x Coh HzHy.o

Rio Rancho - Albuquerque, NM

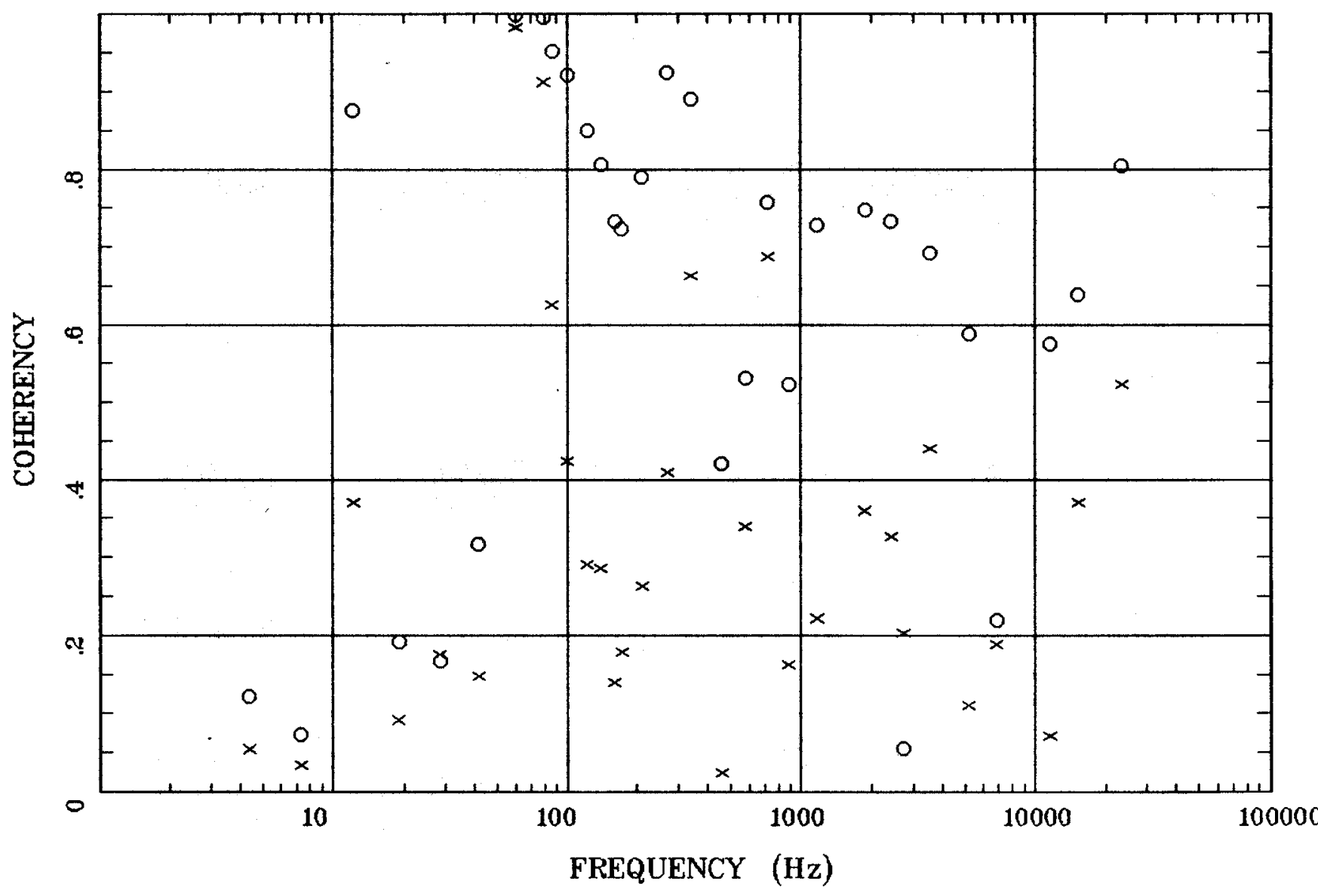

Client:

Remote: Local E

Acquired: 10:3 May 23, 1997

Survey Co:USGS GD-MRP Denver
Rotation:

Filename: rr02a1.all

Channels: Ch1 Ch2 Ch3 Ch4 Ch5 Ch6 Ch7

Plotted: 15:16 Mar 08, 2001

<EMI - ElectroMagnetic Instruments > 
Station 3

APPARENT RESISTIVITY

Rio Rancho - Albuquerque, NM

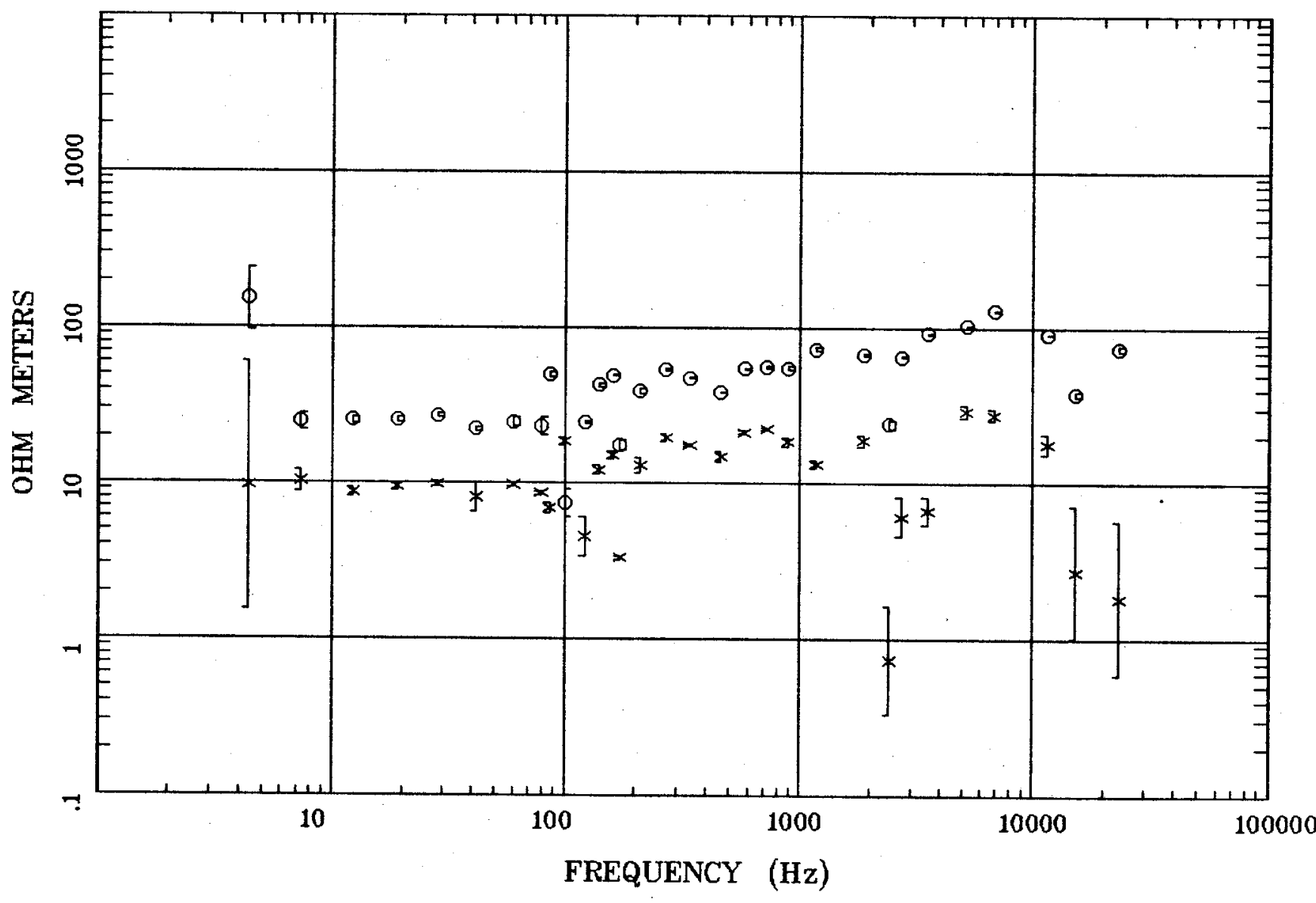

Client:

Remote: Local B

Acquired: 14:4 May 17, 1997 Survey Co:USGS GD-MRP Denver
Rotation:

Filename: rro3h.all

Channels: Ch1 Ch2 Ch3 Ch4 Ch5 Ch8 Ch9

Plotted: 15:17 Mar 08, 2001

< EMI - ElectroMagnetic Instruments > 


\section{Station 3}

IMPEDANCE PHASE

Rio Rancho - Albuquerque, NM

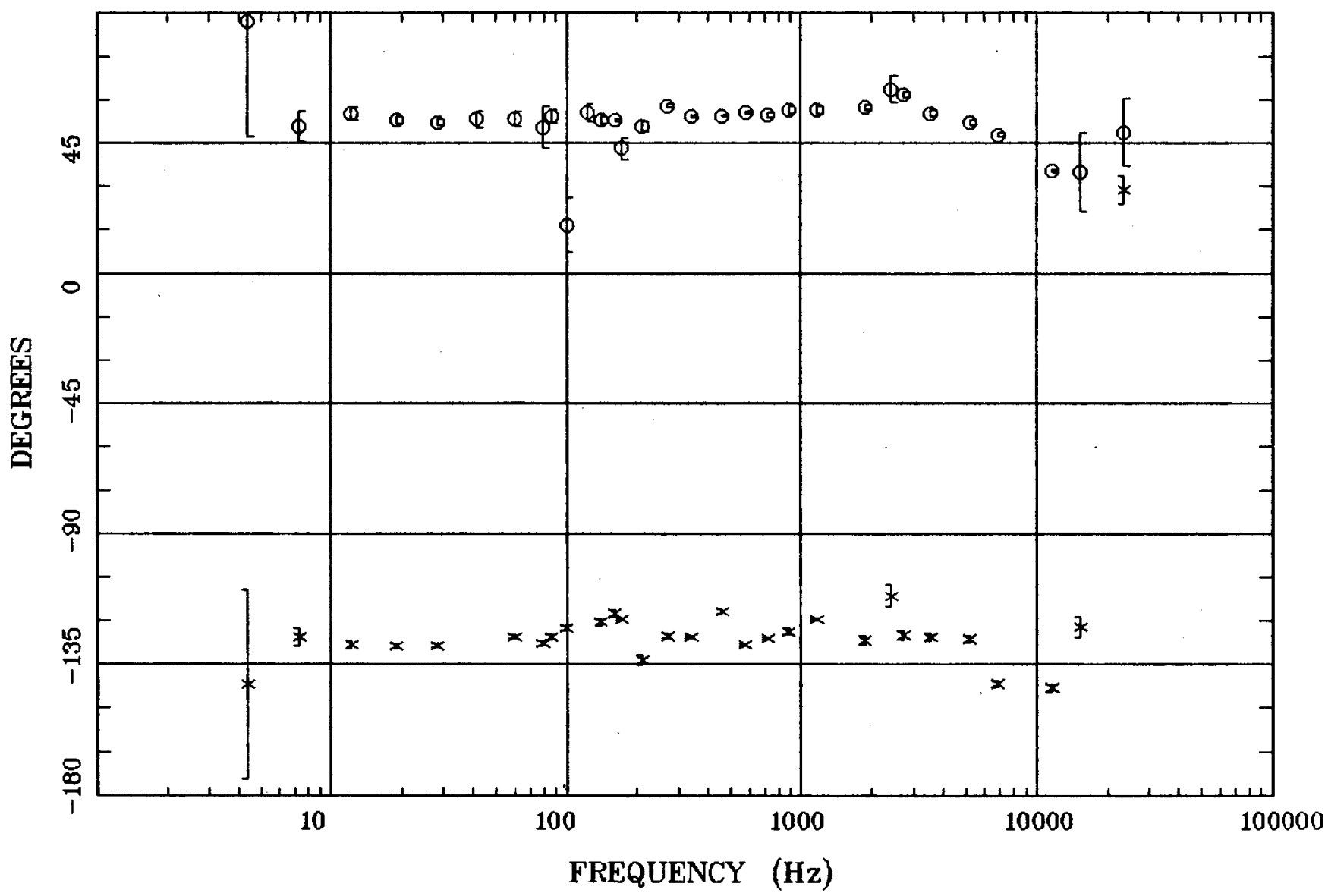

Client:

Remote: Local $B$

Acquired: 14:4 May 17, 1997

Survey Co:USGS GD-MRP Denver
Rotation:

Filename: rro3h.all

Channels: Ch1 Ch2 Ch3 Ch4 Ch5 Ch8 Ch9

Plotted: 15:17 Mar 08, 2001

< EMI - ElectroMagnetic Instruments > 
Station 3

ROTATION ANGLE

Rio Rancho - Albuquerque, NM

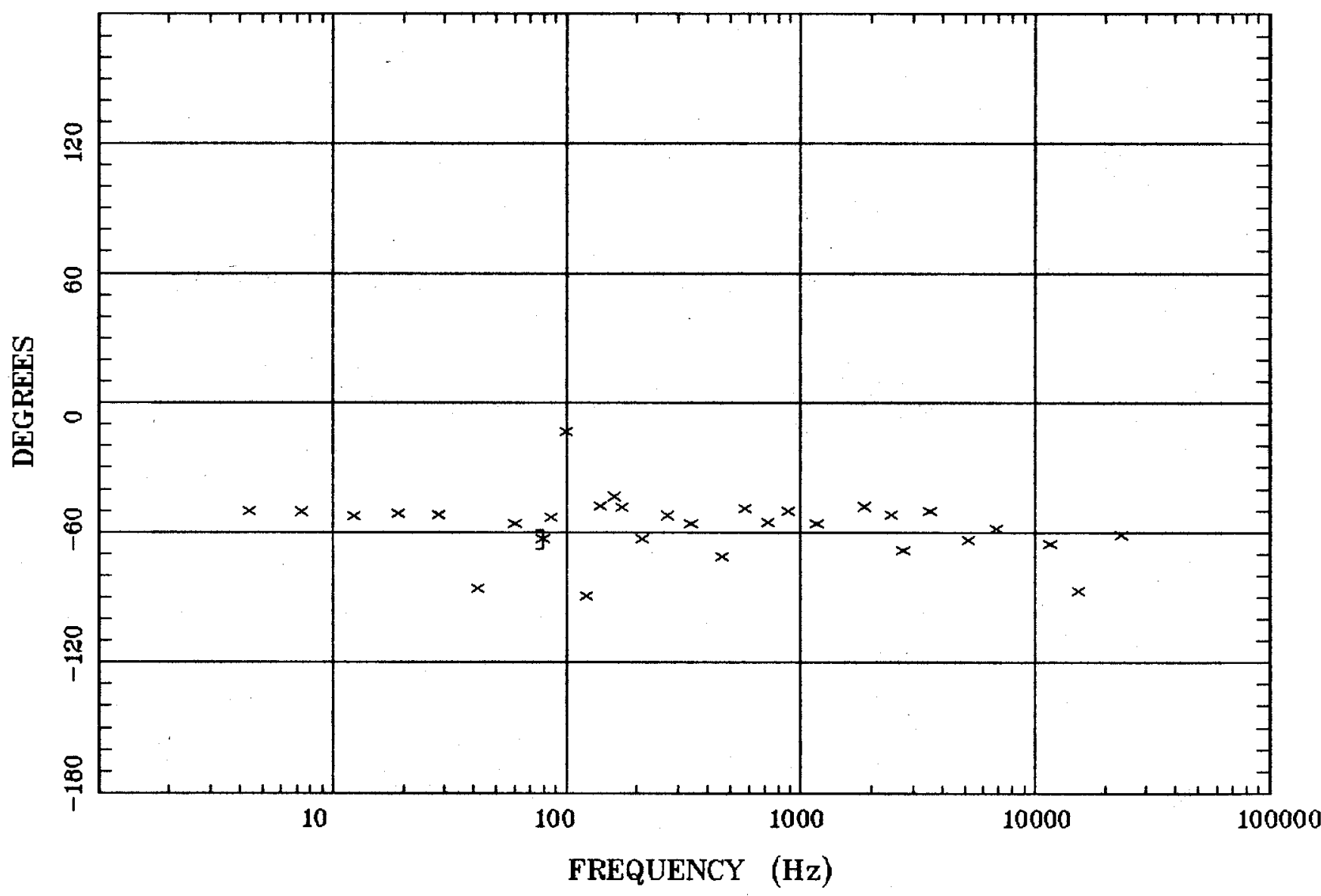

Client:

Remote: Local B

Acquired: 14:4 May 17, 1997

Survey Co:USGS GD-MRP Denver
Rotation:

Filename: rro3h.all

Channels: Ch1 Ch2 Ch3 Ch4 Ch5 Ch8 Ch9

Plotted: 15:17 Mar 08, 2001

< EMI - ElectroMagnetic Instruments > 
Station 3

IMPEDANCE SKEW

Rio Rancho - Albuquerque, NM

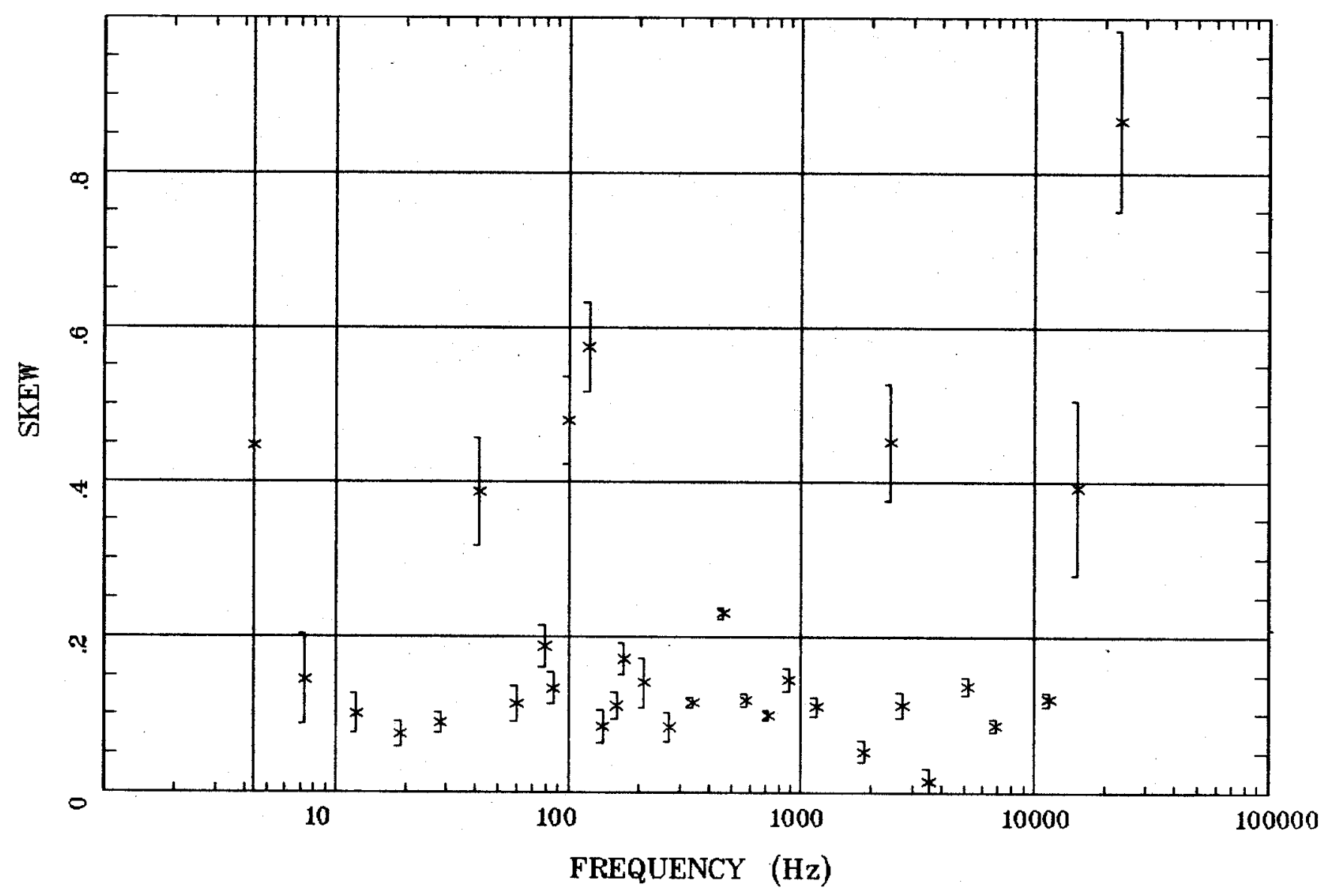

Client:

Remote: Local B

Acquired: 14:4 May 17, 1997

Rotation:

Filename: rro3h.all

Survey Co:USGS GD-MRP Denver

Channels: Ch1 Ch2 Ch3 Ch4 Ch5 Ch8 Ch9

Plotted: 15:17. Mar 08, 2001

$<$ EMI - ElectroMagnetic Instruments 


\section{Station 3}

E MULT Coh.

Rio Rancho - Albuquerque, NM

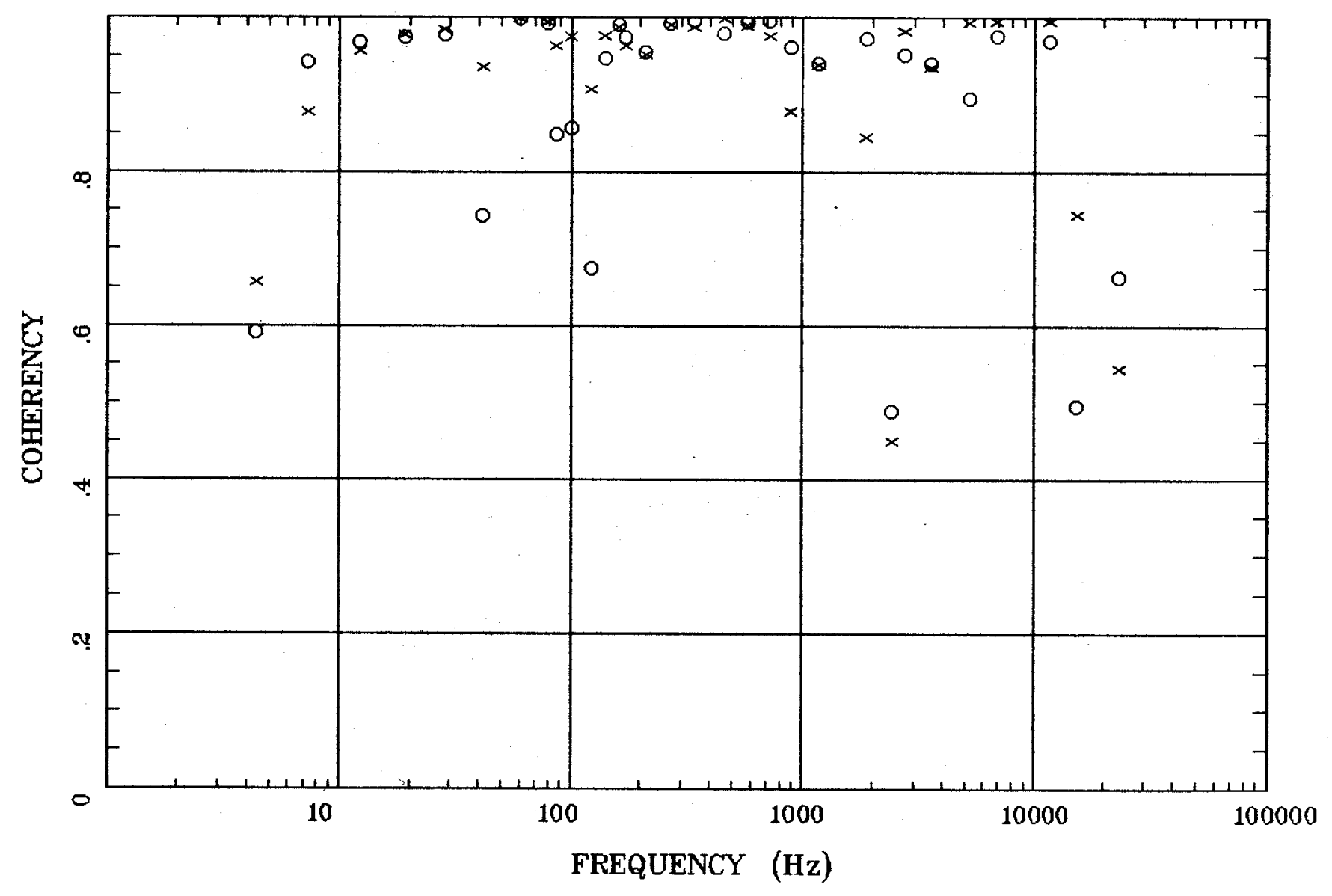

Client:

Remote: Local B

Acquired: 14:4 May 17, 1997

Survey Co:USGS GD-MRP Denver
Rotation:

Filename: rro3h.all

Channels: Ch1 Ch2 Ch3 Ch4 Ch5 Ch8 Ch9

Plotted: 15:17 Mar 08, 2001

< EMI - ElectroMagnetic Instruments > 
Station 3

\section{POLAR PLOTS}

Rio Rancho - Albuquerque, NM

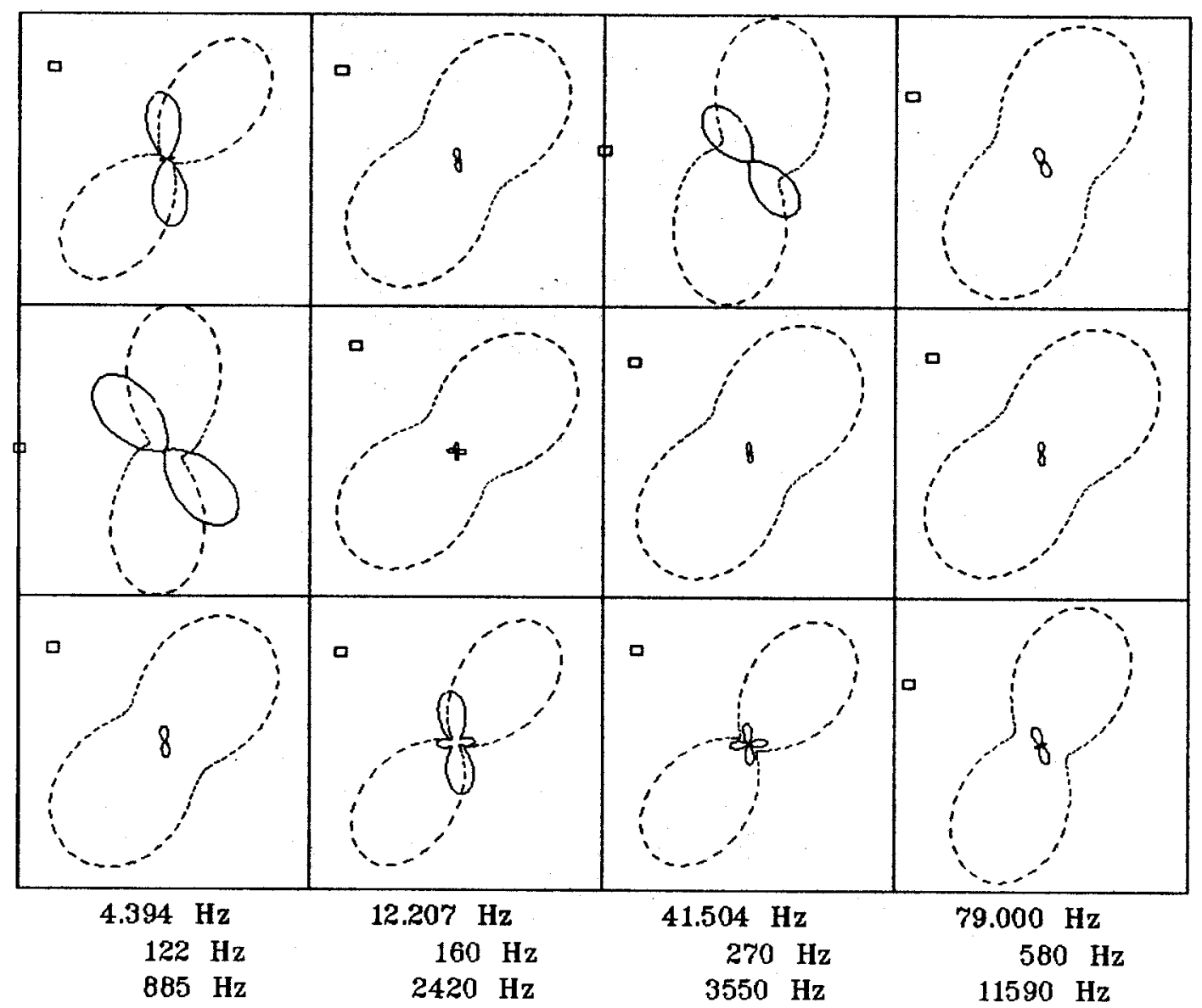

Client:

Remote: Local B

Acquired: 14:4 May 17, 1997 Survey Co:USGS GD-MRP Denver
Rotation:

Filename: rro3h.all

Channels: Ch1 Ch2 Ch3 Ch4 Ch5 Ch8 Ch9

Plotted: 15:17 Mar 08, 2001

$<$ EMI - ElectroMagnetic Instruments 
TIPPER MAGNITUDE

Rio Rancho - Albuquerque, NM

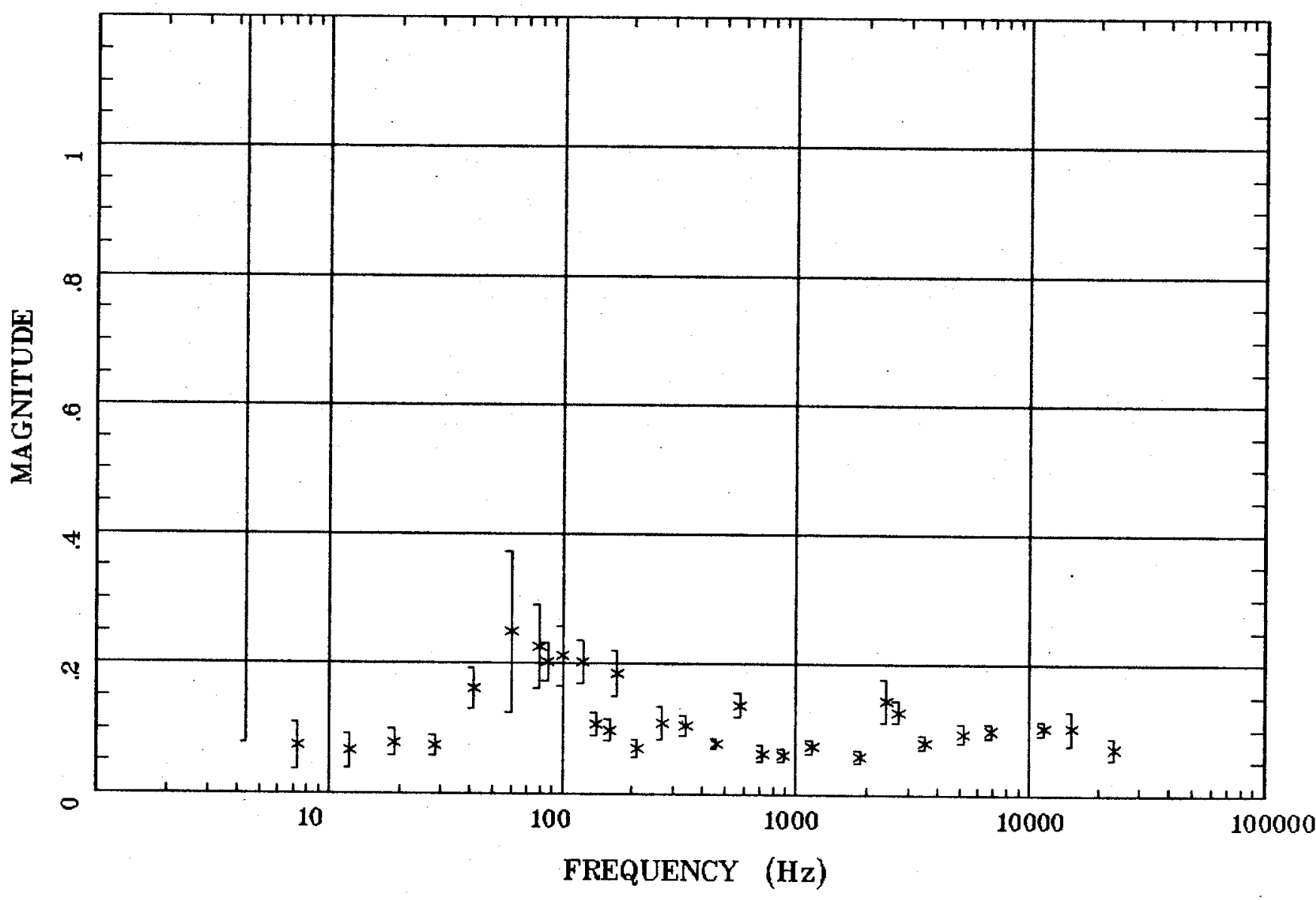

Client:

Remote: Local $B$

Acquired: 14:4 May 17, 1997 Survey Co:USGS GD-MRP Denver
Rotation:

Filename: rro3h.all

Channels: Ch1 Ch2 Ch3 Ch4 Ch5 Ch8 Ch9

Plotted: 15:17 Mar 08, 2001

< EMI - ElectroMagnetic Instruments > 
TIPPER STRIKE

Rio Rancho - Albuquerque, NM

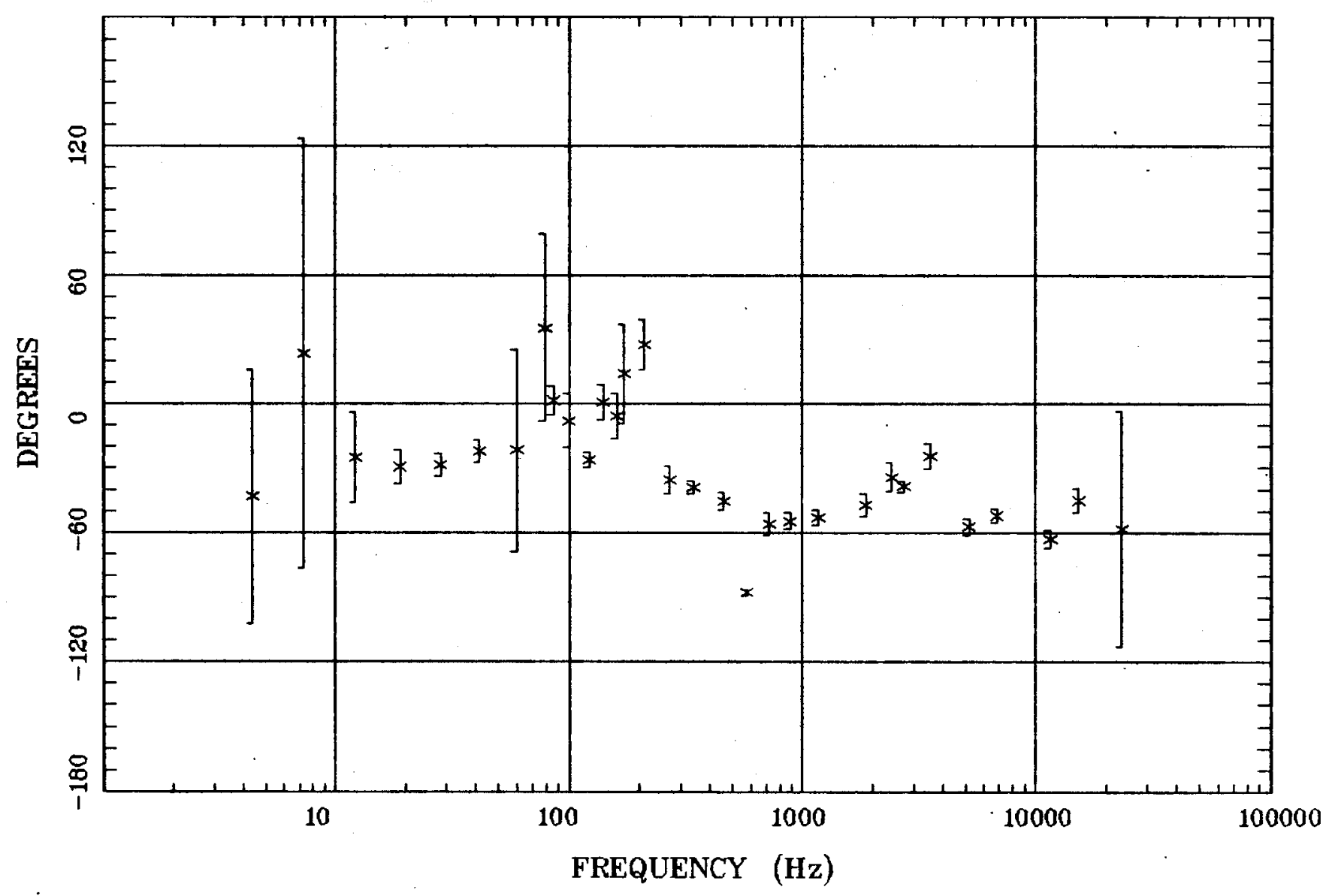

Client:

Remote: Local $B$

Acquired: 14:4 May 17, 1997 Survey Co:USGS GD-MRP Denver
Rotation:

Filename: rro3h.all

Channels: Ch1 Ch2 Ch3 Ch4 Ch5 Ch8 Ch9

Plotted: 15:17 Mar 08, 2001

< EMI - ElectroMagnetic Instruments > 
HzHx.x Coh HzHy.o

Rio Rancho - Albuquerque, NM

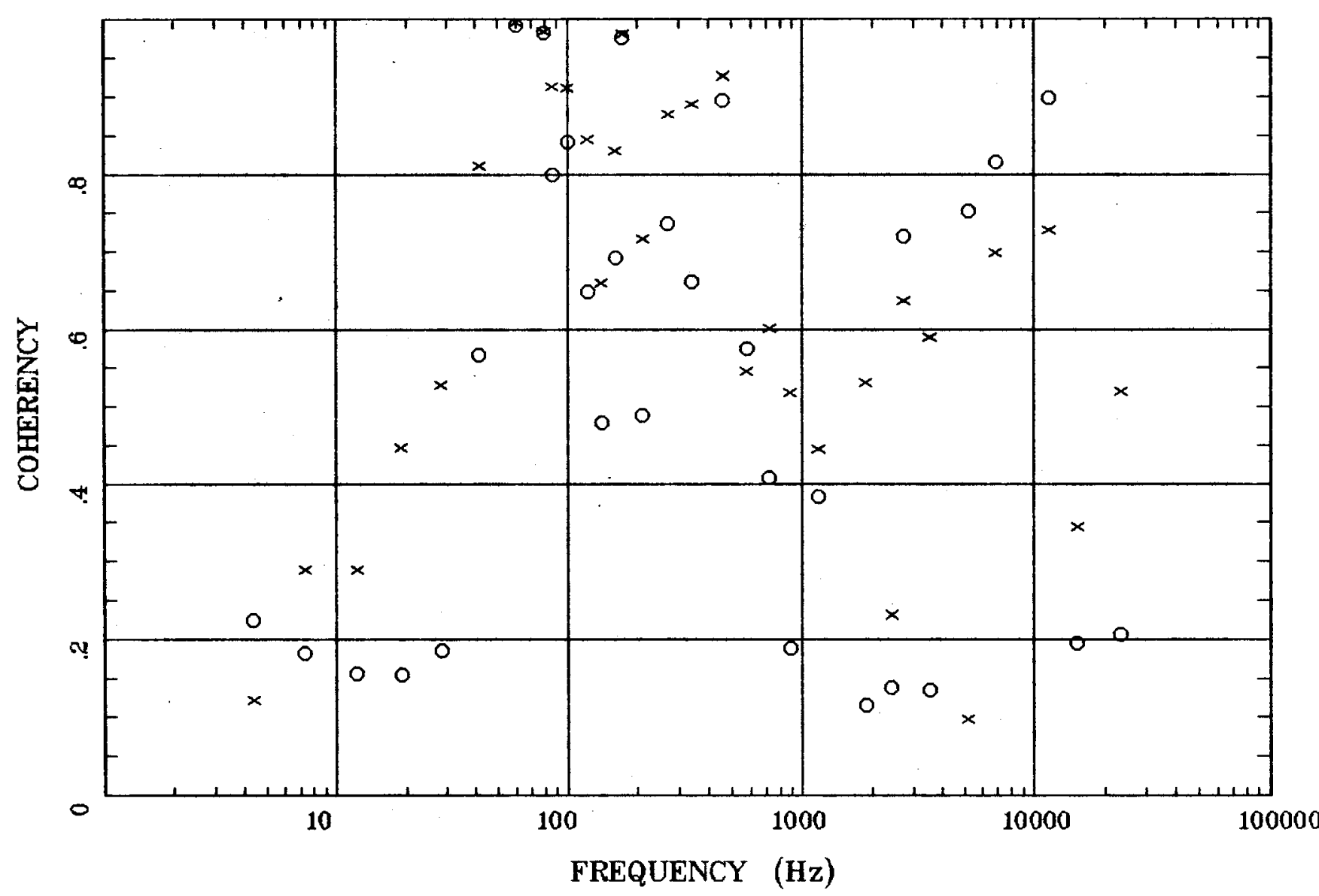

Client:

Remote: Local B

Acquired: 14:4 May 17, 1997 Survey Co:USGS GD-MRP Denver
Rotation:

Filename: rro3h.all

Channels: Ch1 Ch2 Ch3 Ch4 Ch5 Ch8 Ch9 Plotted: 15:17 Mar 08, 2001

< EMI - ElectroMagnetic Instruments > 


\section{Station 4}

APPARENT RESISTIVITY

Rio Rancho - Albuquerque, NM

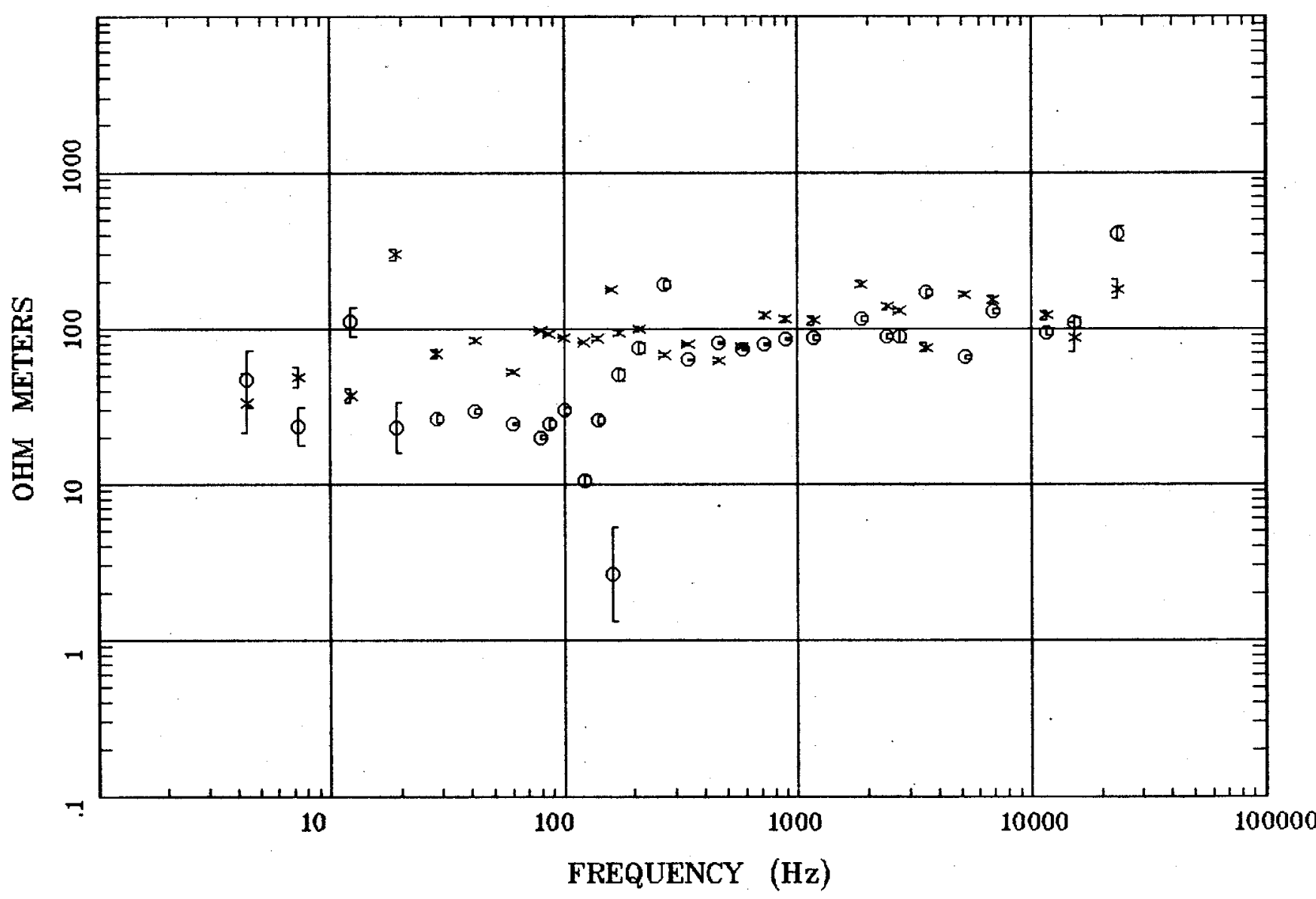

Client:

Remote: Local E

Acquired: 12.5 May 18, 1997 Survey Co:USGS GD-MRP Denver
Rotation:

Filename: rr04.all

Channels: Ch1 Ch2 Ch3 Ch4 Ch5 Ch6 Ch7 Platted: 15:18 Mar 08, 2001

< EMI - ElectroMagnetic Instruments > 


\section{Station 4}

\section{IMPEDANCE PHASE}

Rio Rancho - Albuquerque, NM

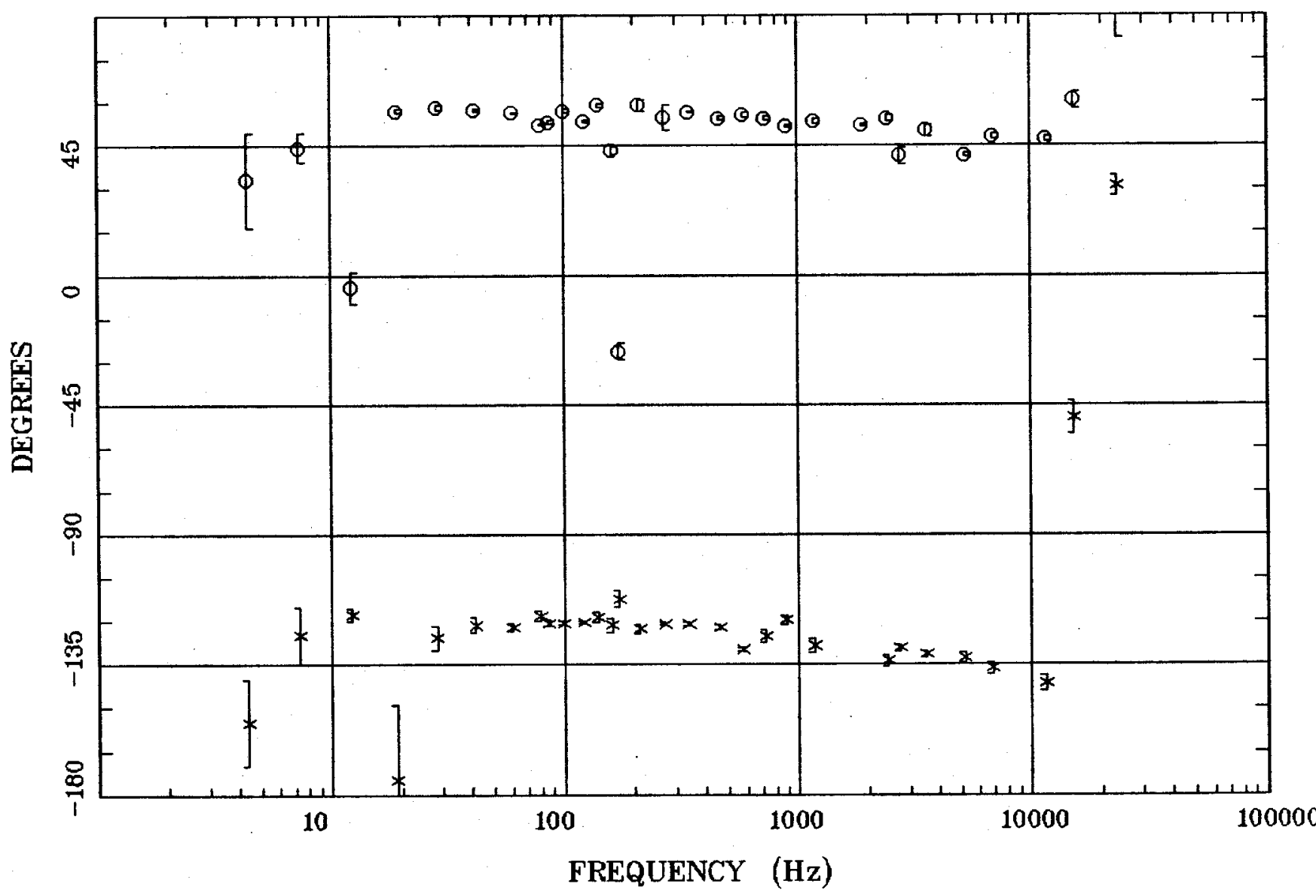

Client:

Remote: Local E

Acquired: 12:5 May 18, 1997

Survey Co:USGS GD-MRP Denver
Rotation:

Filename: rr04.all

Channels: Ch1 Ch2 Ch3 Ch4 Ch5 Ch6 Ch7

Plotted: 15:18 Mar 08, 2001

< EMI - ElectroMagnetic Instruments > 
Station 4

ROTATION ANGLE

Rio Rancho - Albuquerque, NM

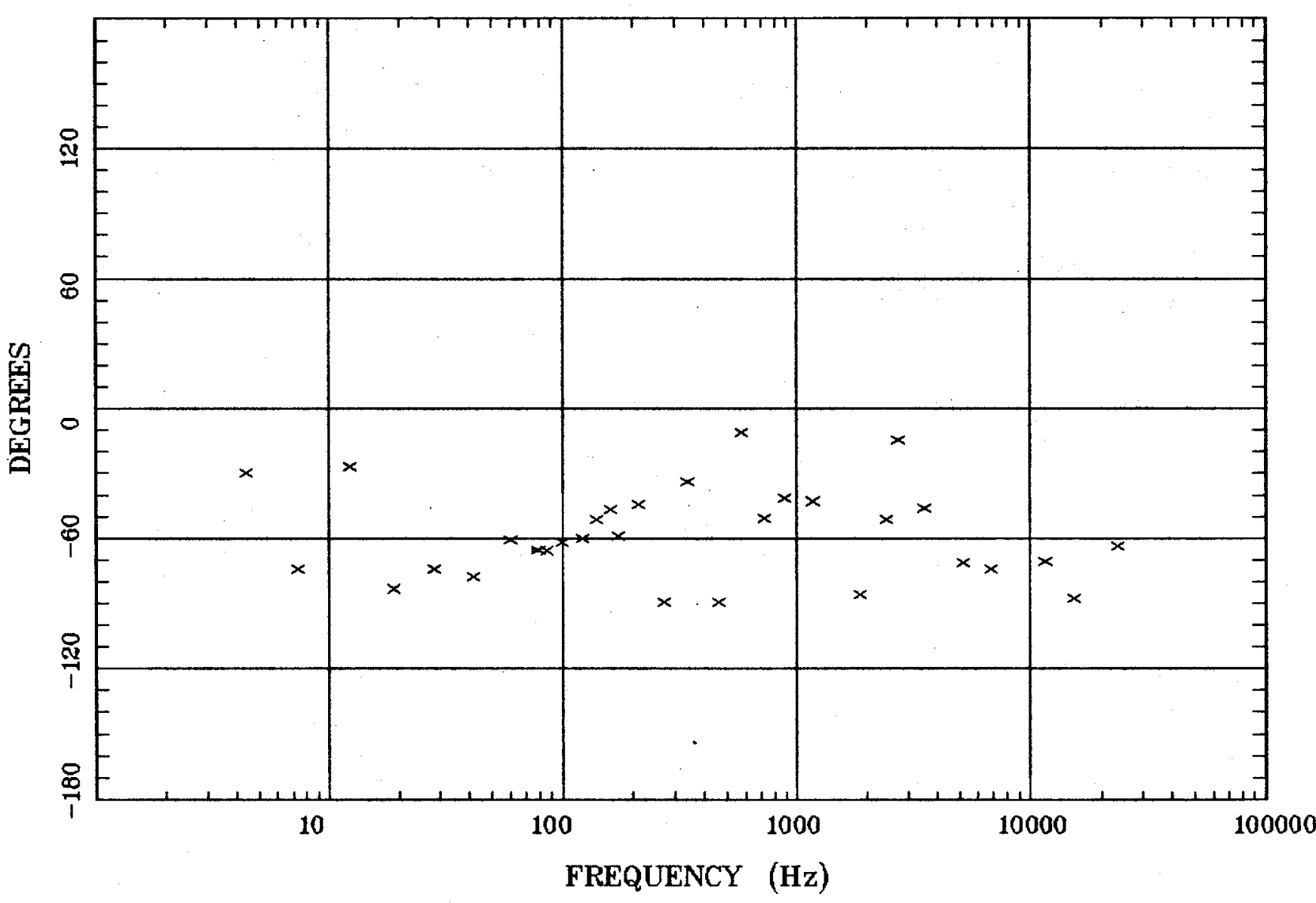

Client:

Remote: Local E Acquired: 12:5 May 18, 1997 Survey Co:USGS GD-MRP Denver

\section{Rotation:}

Filename: rro4.all

Channels: Ch1 Ch2 Ch3 Ch4 Ch5 Ch6 Ch7

Platted: 15:18 Mar 08, 2001

< EMI - ElectroMagnetic Instruments > 
Station 4

IMPEDANCE SKEW

Rio Rancho - Albuquerque, NM

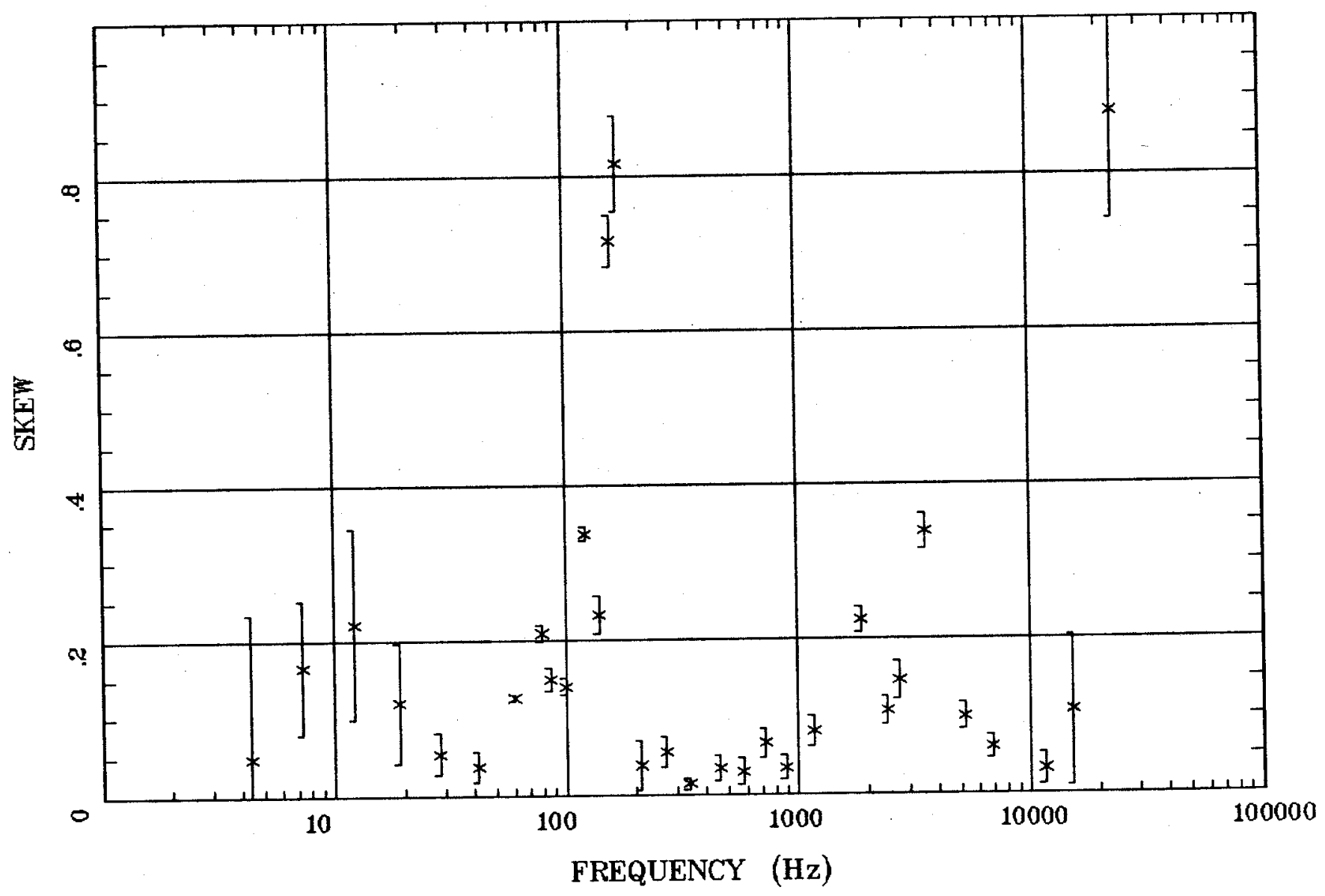

Client:

Remote: Local E

Acquired: 12:5 May 18, 1997

Survey Co:USGS GD-MRP Denver

Rotation:

Filename: rro4.all

Channels: Ch1 Ch2 Ch3 Ch4 Ch5 Ch6 Ch7

Plotted: 15:18 Mar 08, 2001

< EMI - ElectroMagnetic Instruments > 


\section{Station 4}

E MULT Coh.

Rio Rancho - Albuquerque, NM

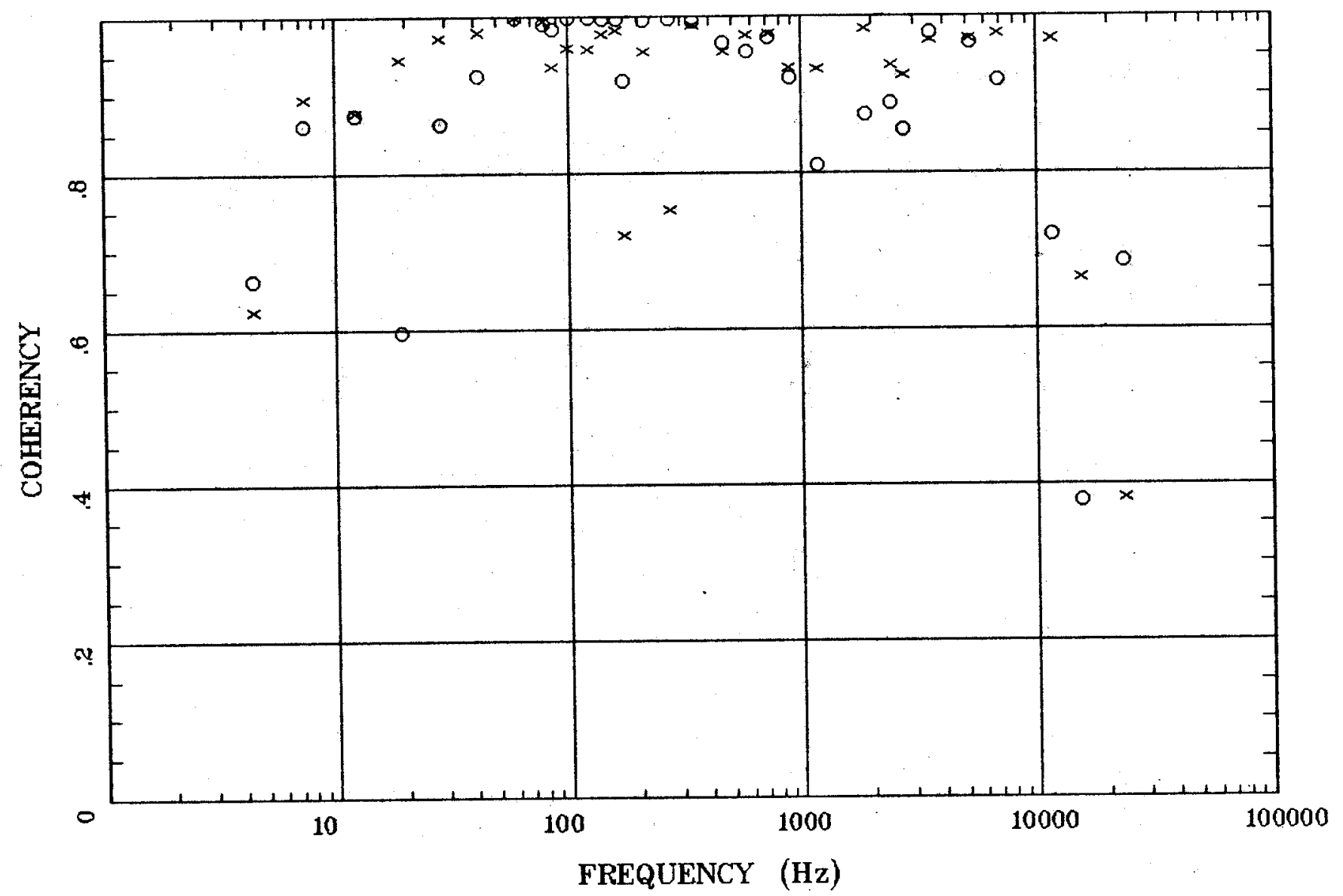

Rotation:

Client:

Remote: Local E

Acquired: 12:5 May 18, 1997 Survey Co:USGS GD-MRP Denver
Filename: rr04.all

Channels: Ch1 Ch2 Ch3 Ch4 Ch5 Ch6 Ch7

Plotted: 15:18 Mar 08, 2001

$\langle$ EMI - ElectroMagnetic Instruments > 


\section{Station 4}

POLAR PLOTS

Rio Rancho - Albuquerque, NM

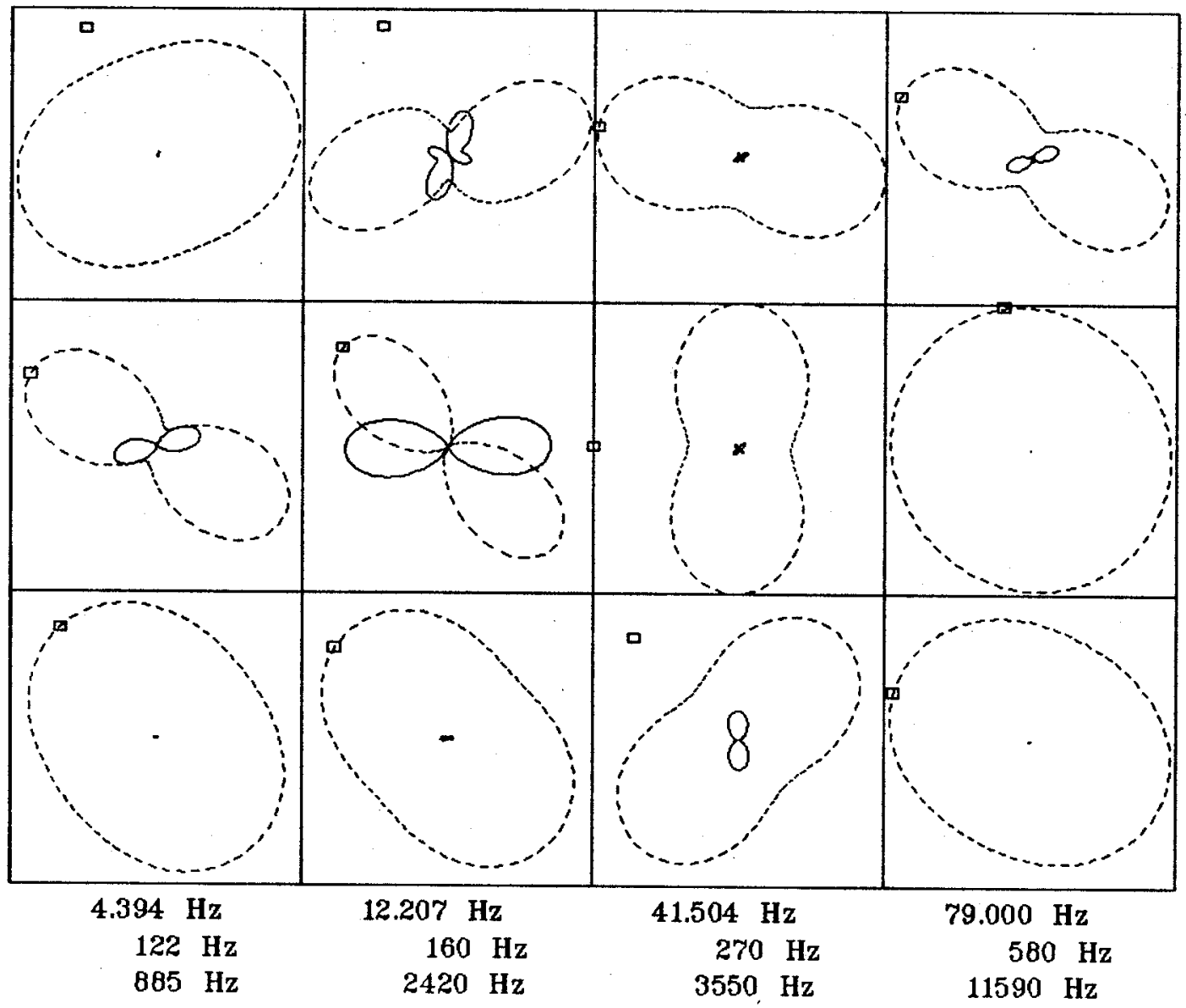

\section{Client:}

Remote: Local E

Acquired: 12:5 May 18, 1997

Survey Co:USGS GD-MRP Denver
Rotation:

Filename: rr04.all

Channels: Ch1 Ch2 Ch3 Ch4 Ch5 Ch6 Ch7

Plotted: 15:18 Mar 08, 2001

$<$ EMI - ElectroMagnetic Instruments 
Rio Rancho - Albuquerque, NM

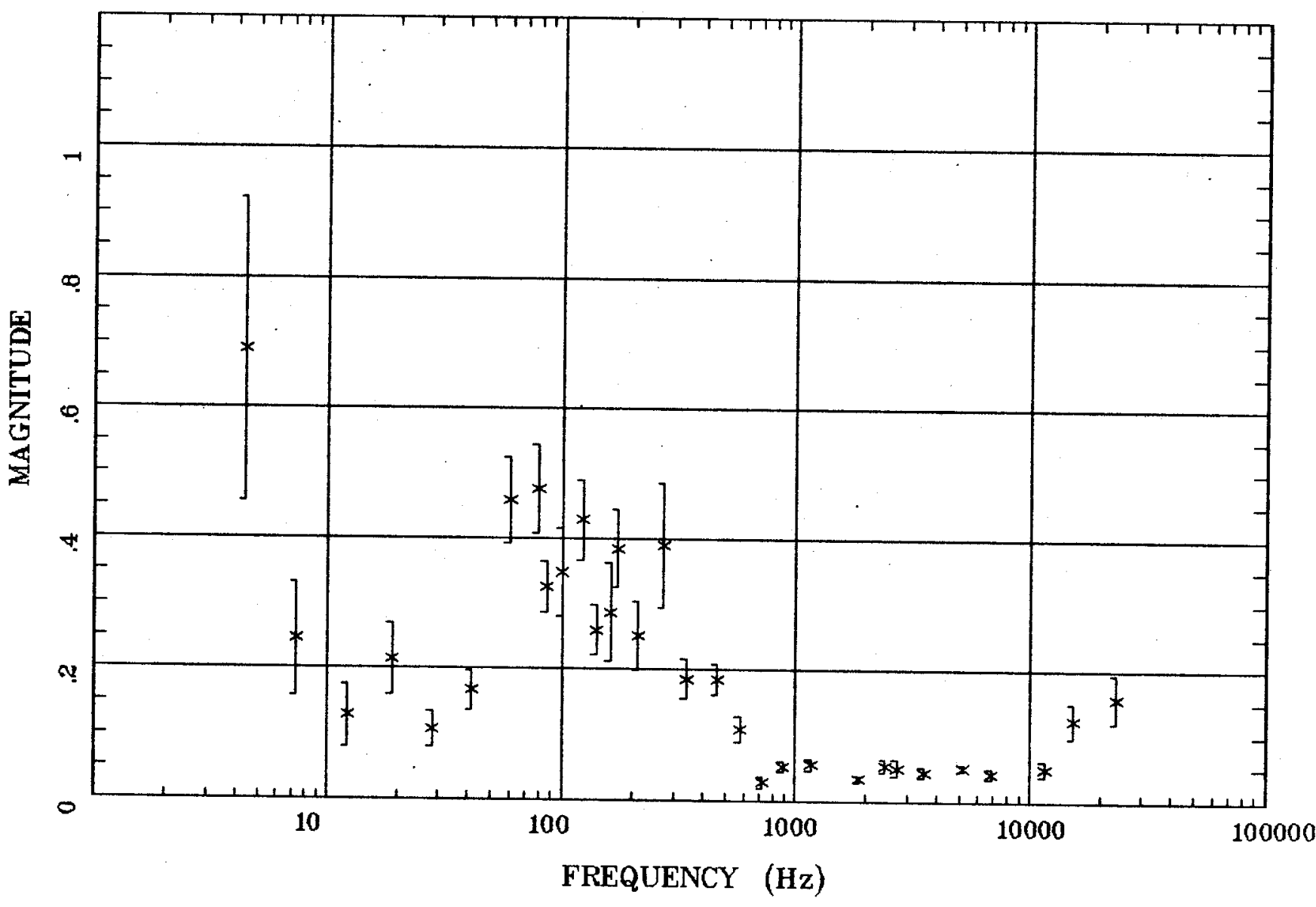

Client:

Remote: Local E

Acquired: 12:5 May 18, 1997

Survey Co:USGS GD-MRP Denver
Rotation:

Filename: rr04.all

Channels: Ch1 Ch2 Ch3 Ch4 Ch5 Ch6 Chr

Plotted: 15:18 Mar 08, 2001

< EMI - ElectroMagnetic Instruments > 


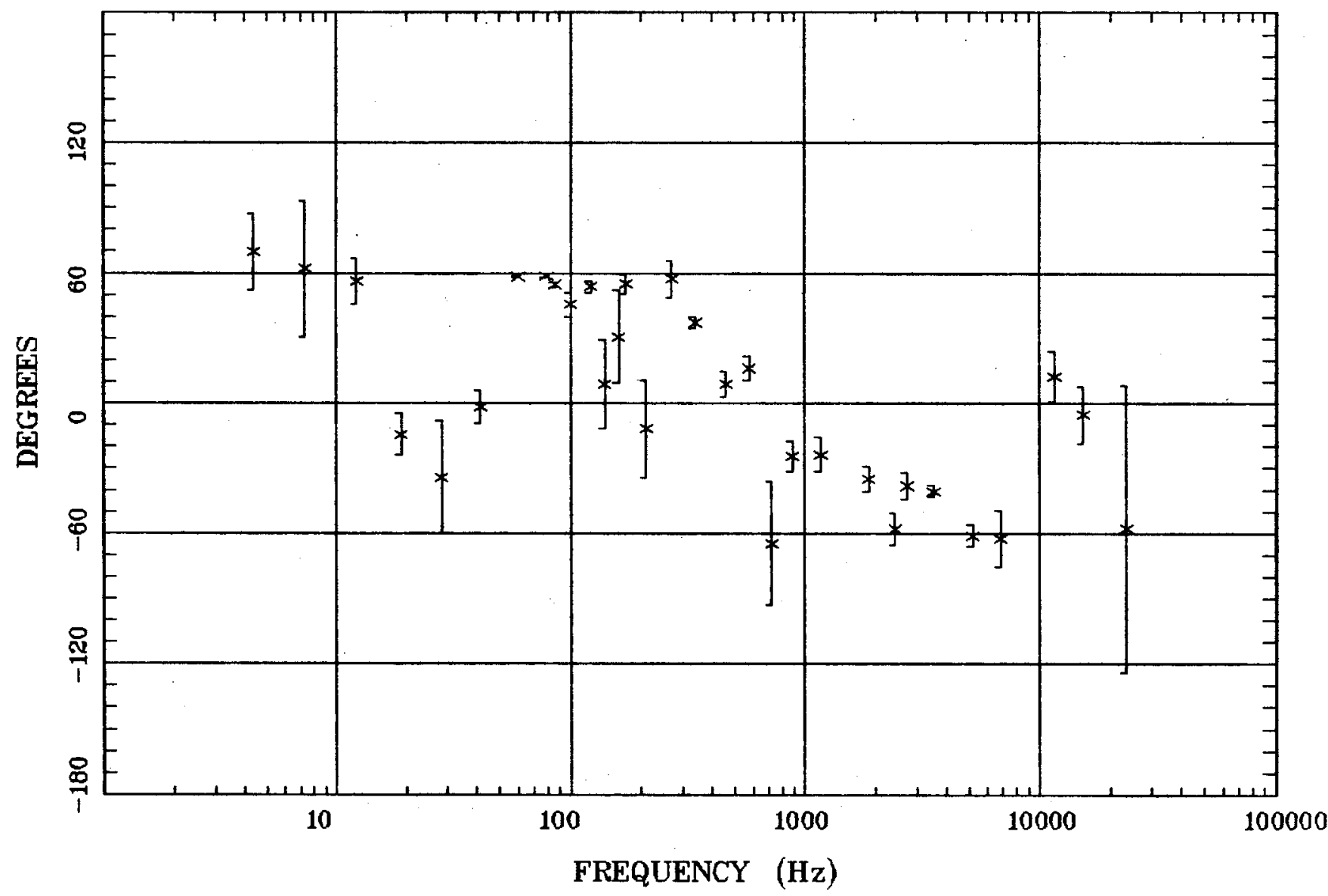

Client:

Remote: Local E Acquired: 12:5 May 18, 1997 Survey Co:USGS GD-MRP Denver
Rotation:

Filename: rr04.all

Channels: Ch1 Ch2 Ch3 Ch4 Ch5 Ch6 Ch7

Plotted: 15:18 Mar 08, 2001

$<$ EMI - ElectroMagnetic Instruments 
Station 4

HzHx.x Coh HzHy.o

Rio Rancho - Albuquerque, NM

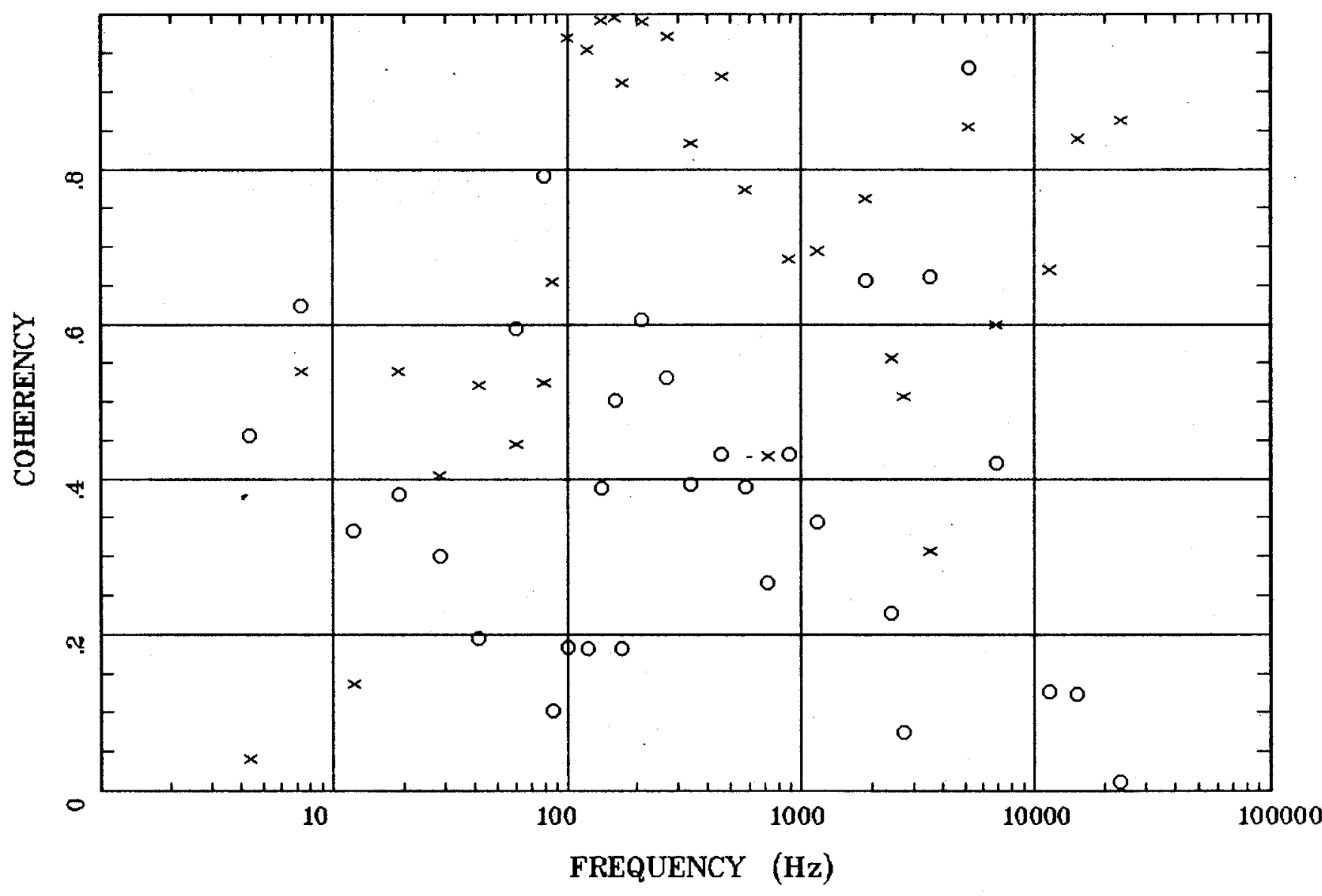

Client:

Remote: Local E Acquired: 12:5 May 18, 1997 Survey Co:USGS GD-MRP Denver
Rotation:

Filename: rr04.all

Channels: Ch1 Ch2 Ch3 Ch4 Ch5 Ch6 Ch7 Plotted: 15:18 Mar 08, 2001

< EMI - ElectroMagnetic Instruments > 
Station 6

APPARENT RESISTIVITY

Rio Rancho - Albuquerque, NM

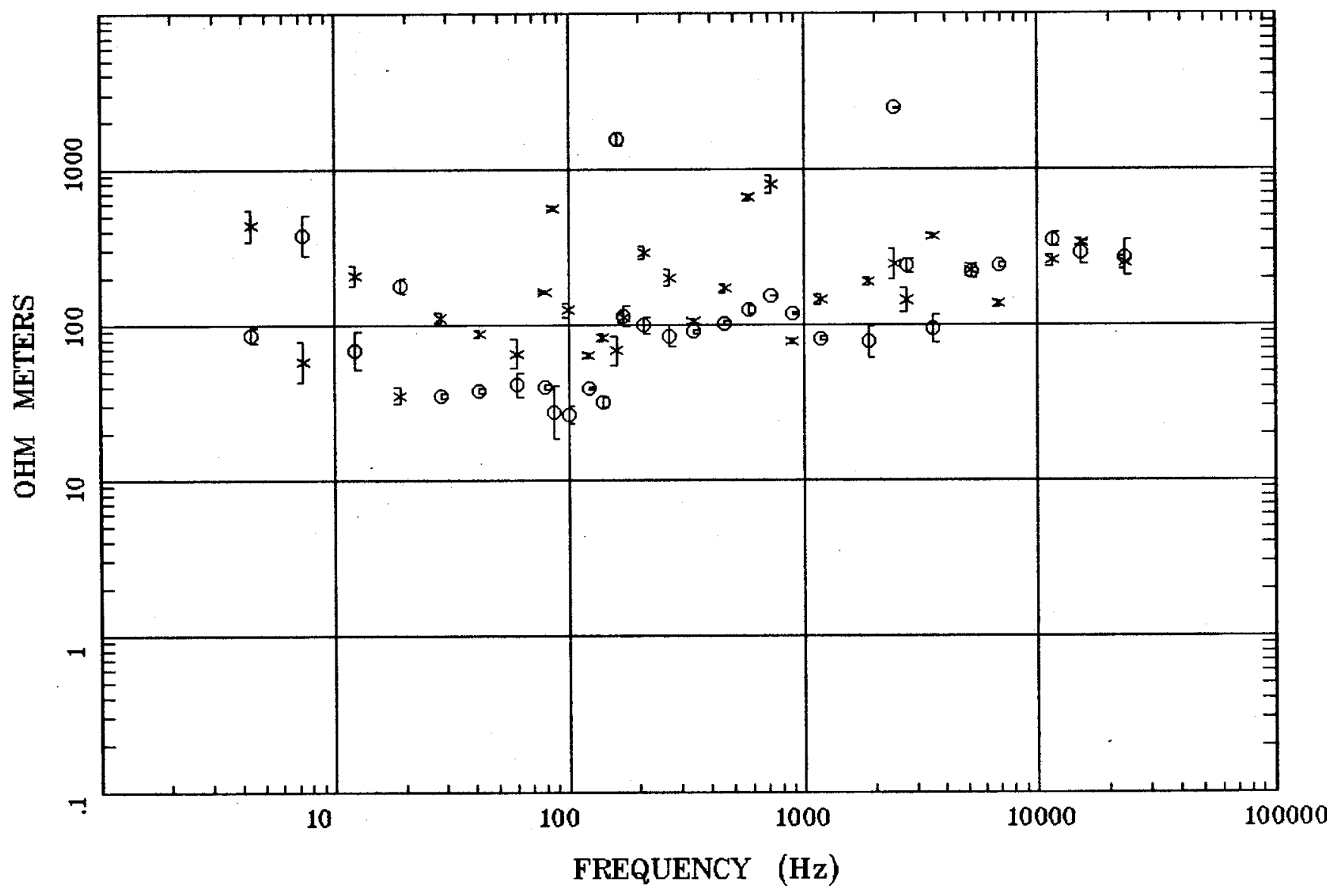

Client:

Remote: Local $\mathrm{E}$

Acquired: 16:1 May 22, 1997

Survey Co:USGS GD-MRP Denver
Rotation:

Filename: rro6e4.all

Channels: Ch1 Ch2 Ch3 Ch4 Ch5 Ch6 Chr

Plotted: 15:18 Mar 08, 2001

< EMI - ElectroMagnetic Instruments > 
Station 6

IMPEDANCE PHASE

Rio Rancho - Albuquerque, NM

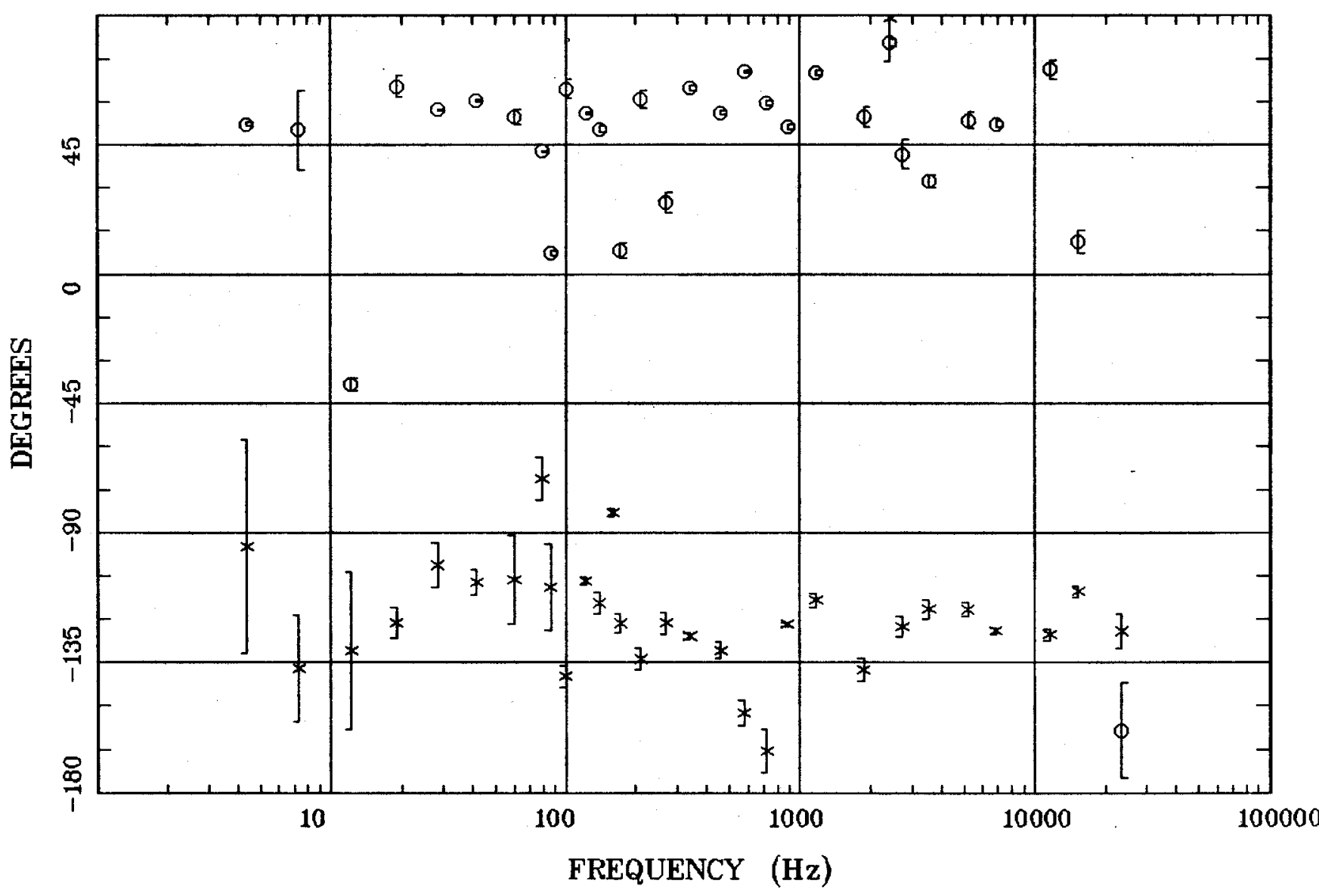

Client:

Remote: Local $\mathrm{E}$

Acquired: 16:1 May 22, 1997 Survey Co:USGS GD-MRP Denver
Rotation:

Filename: rr06e4.all

Channels: Ch1 Ch2 Ch3 Ch4 Ch5 Ch6 Ch7 Plotted: 15:18 Mar 08, 2001

< EMI - ElectroMagnetic Instruments 
Rio Rancho - Albuquerque, NM

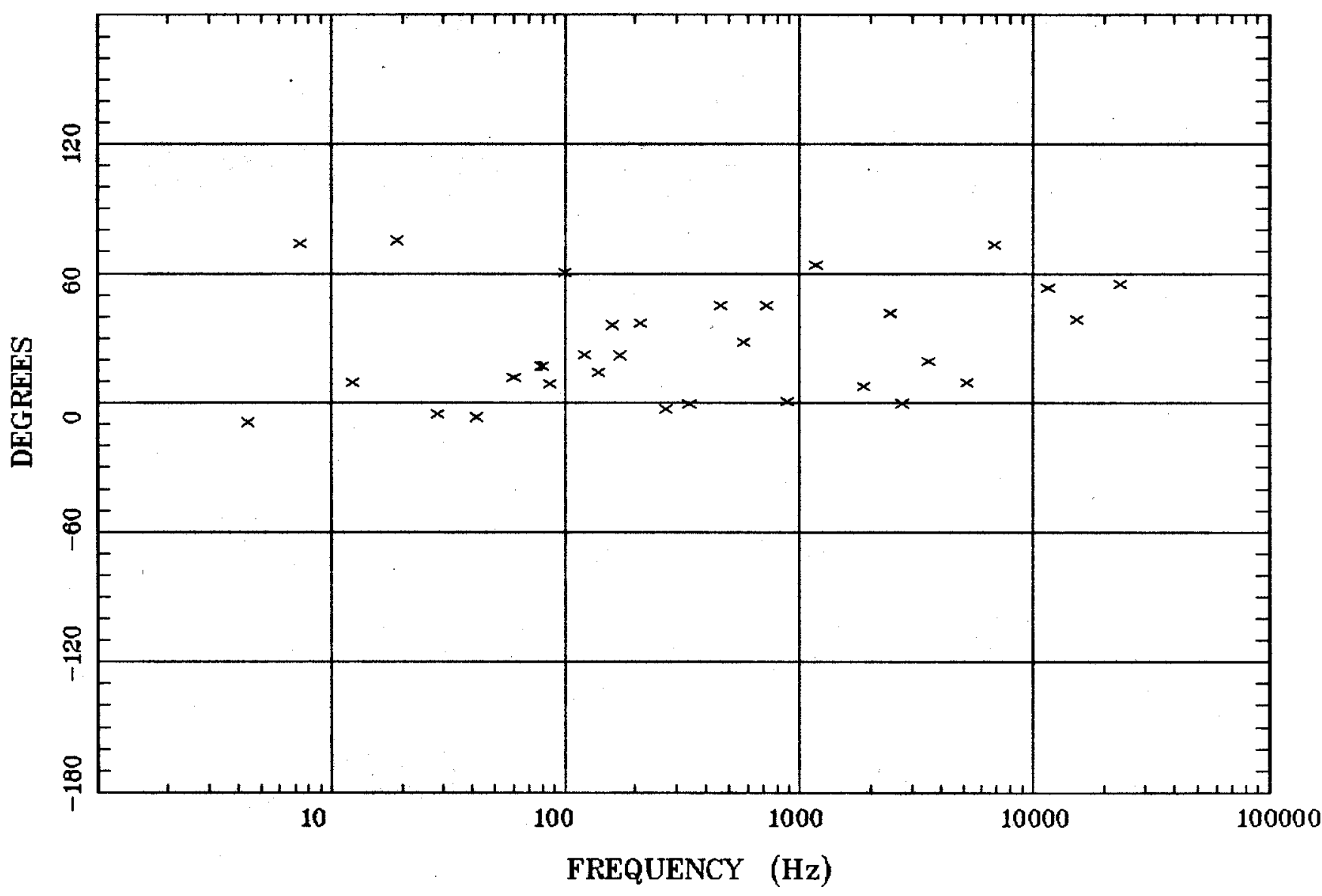

Client:

Remote: Local $\mathrm{E}$

Acquired: 16:1 May 22, 1997 Survey Co:USGS GD-MRP Denver
Rotation:

Filename: rro6e4.all

Channels: Ch1 Ch2 Ch3 Ch4 Ch5 Ch6 Ch7 Plotted: 15:18 Mar 08, 2001

< EMI - ElectroMagnetic Instruments 
Station 6

IMPEDANCE SKEW

Rio Rancho - Albuquerque, NM

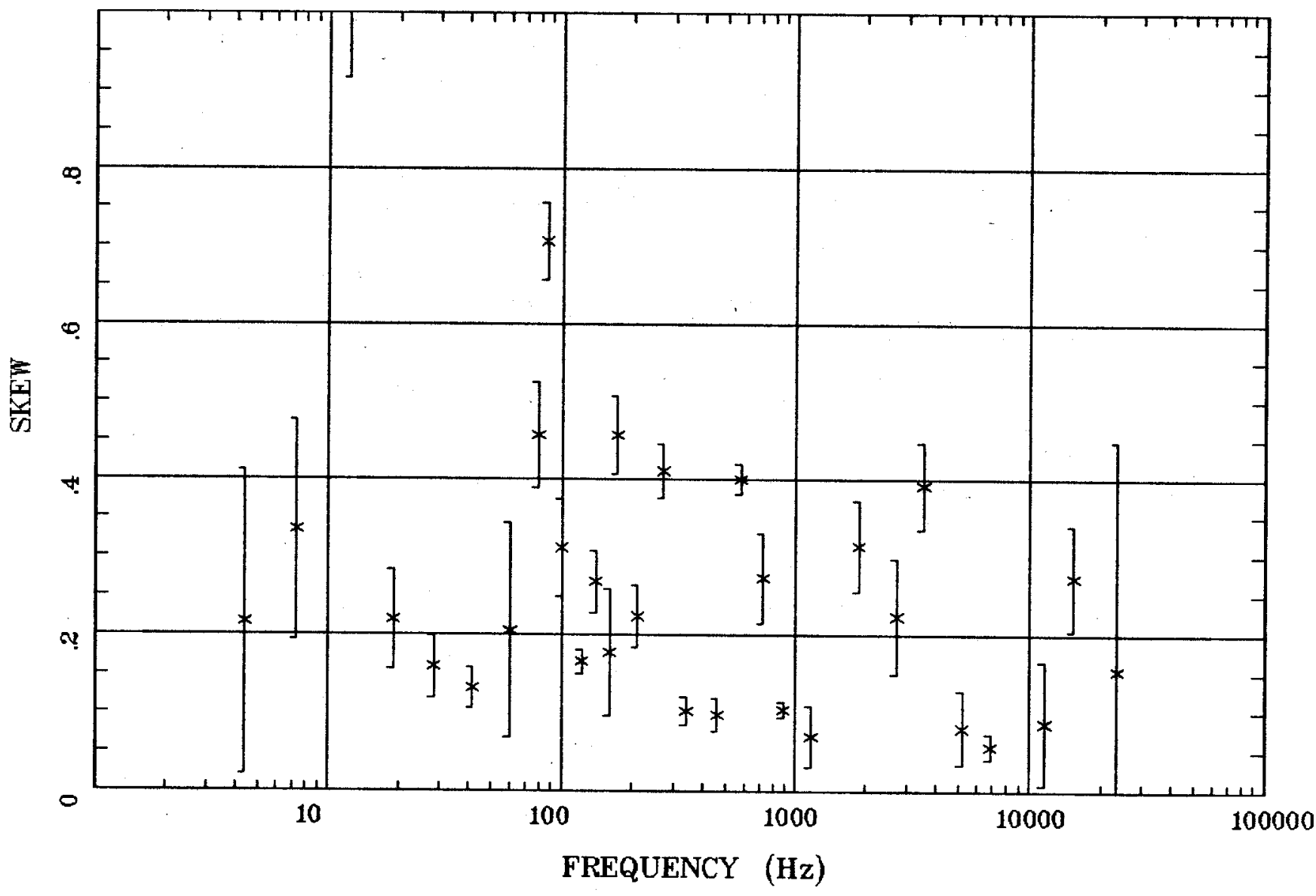

Client:

Remote: Local E

Acquired: 16:1 May 22, 1997

Survey Co:USGS GD-MRP Denver
Rotation:

Filenarne: rro6e4.all

Channels: Ch1 Ch2 Ch3 Ch4 Ch5 Ch6 Ch7

Plotted: 15:18 Mar 08, 2001

$<$ EMI - ElectroMagnetic Instruments 


\section{Station 6}

E MULT Coh.

Rio Rancho - Albuquerque, NM

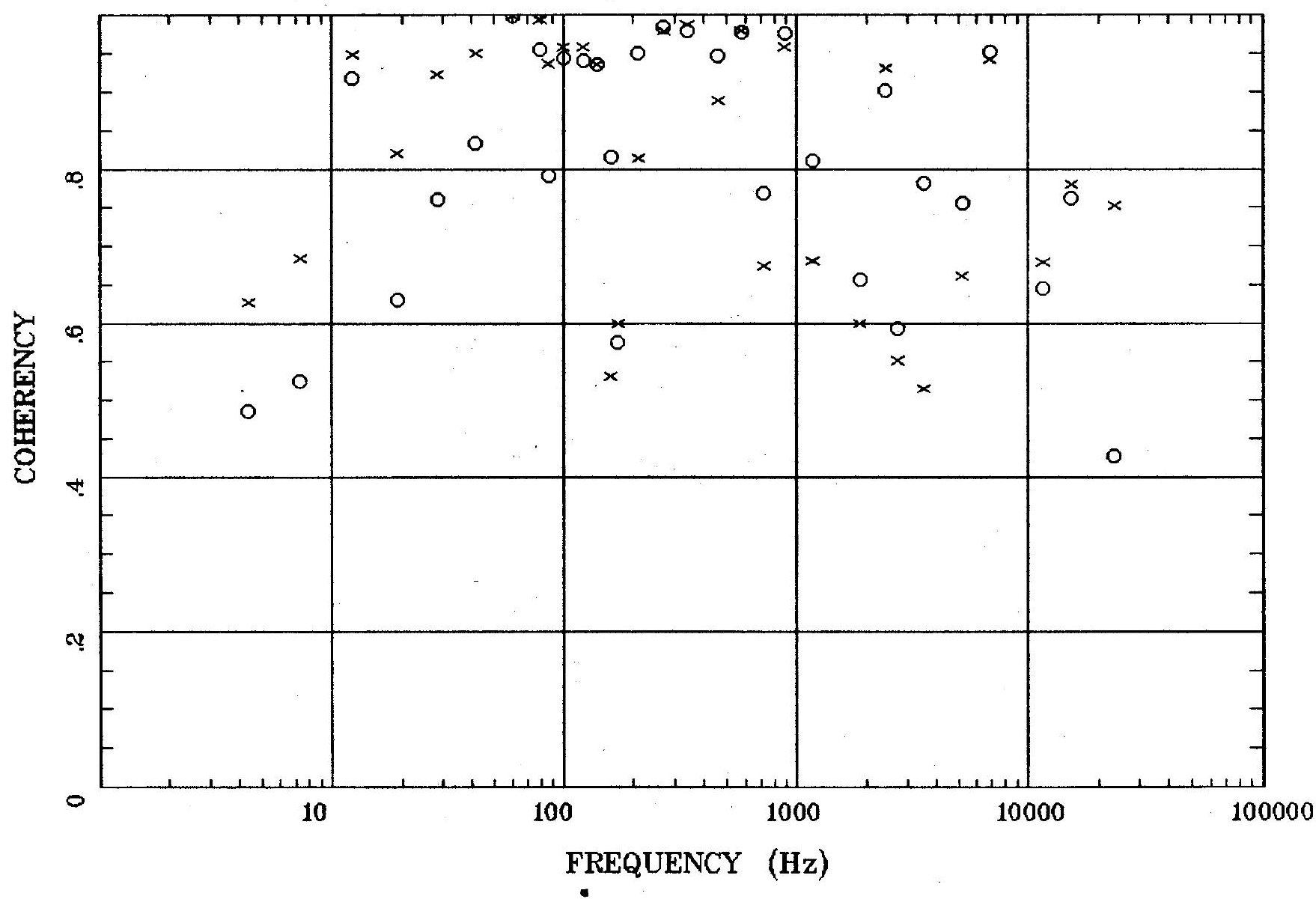

Client:

Remote: Local E Acquired: 16:1 May 22, 1997 Survey Co:USGS GD-MRP Denver
Rotation:

Filename: rro6e4.all

Channels: Ch1 Ch2 Ch3 Ch4 Ch5 Ch6 Ch7

Plotted: 15:18 Mar 08, 2001.

< EMI - ElectroMagnetic Instruments > 


\section{POLAR PLOTS}

Rio Rancho - Albuquerque, NM

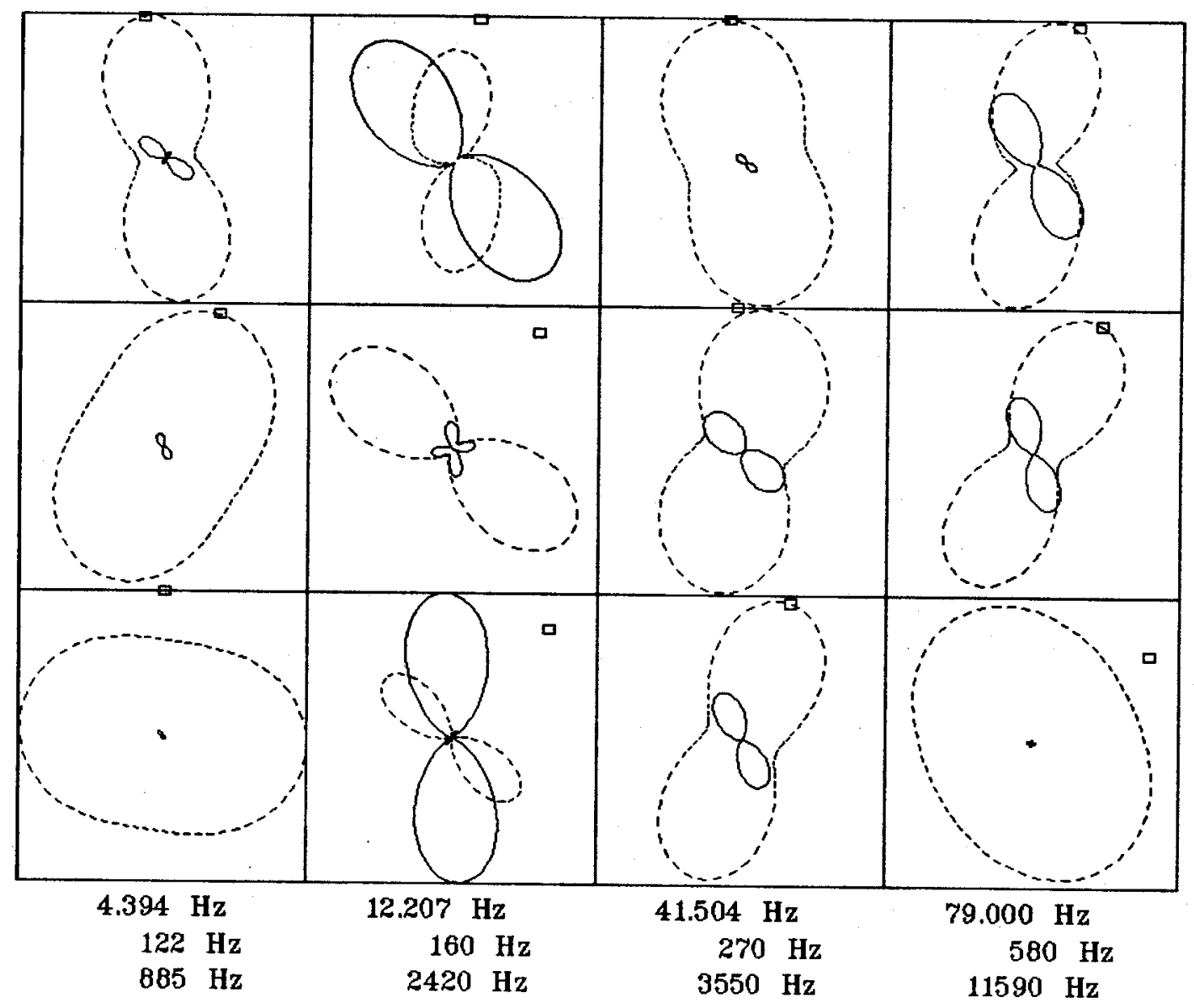

Client:

Remote: Local E

Acquired: 16:1 May 22, 1997

Survey Co:USGS GD-MRP Denver
Rotation:

Filename: rro6e4.all

Channels: Ch1 Ch2 Ch3 Ch4 Ch5 Ch6 Ch7

Plotted: 15:18 Mar 08, 2001

$<$ EMI - ElectroMagnetic Instruments > 


\section{Station 6}

Rio Rancho - Albuquerque, NM

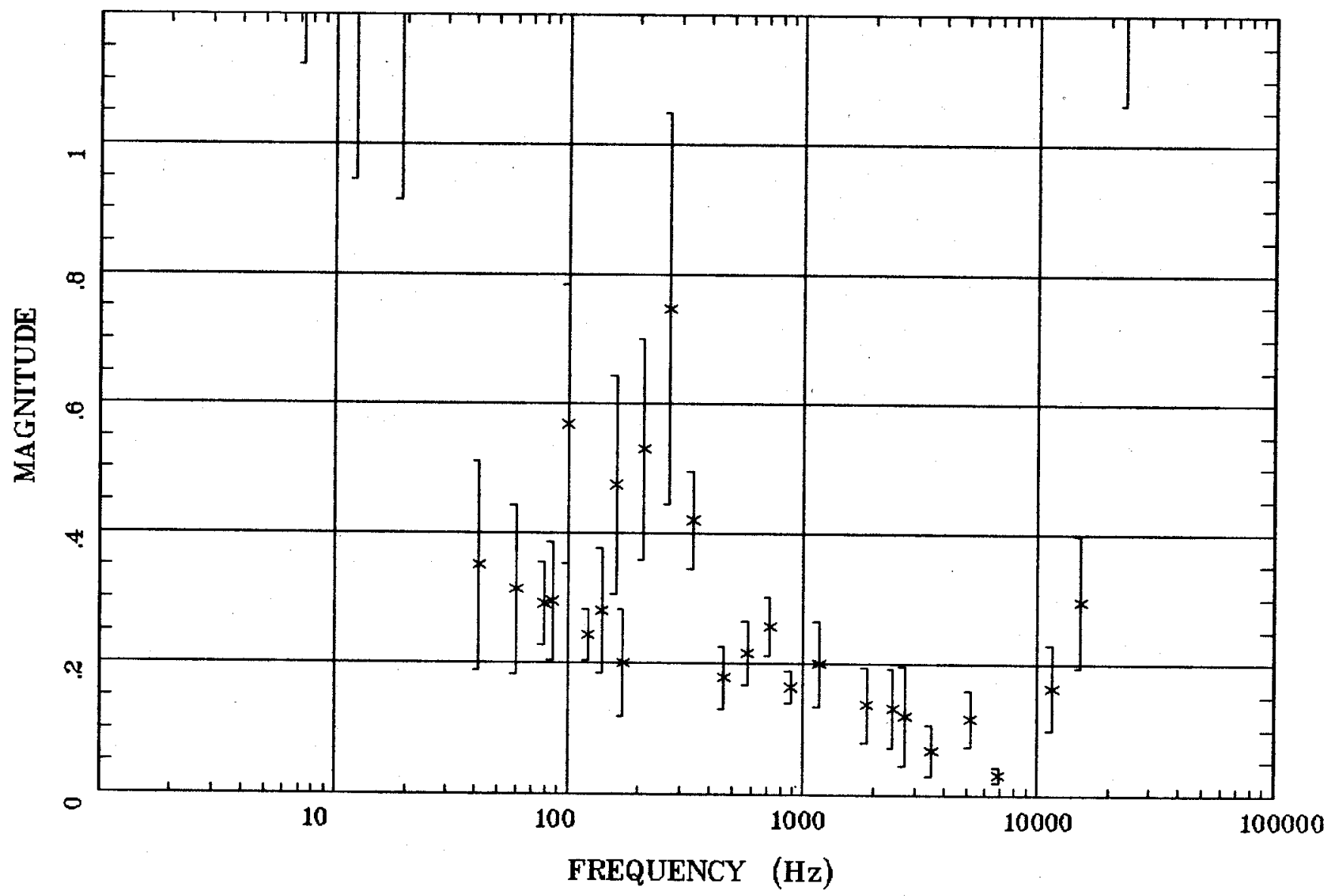

Client:

Remote: Lacal E

Acquired: 16:1 May 22, 1997

Survey Co:USGS GD-MRP Denver
Rotation:

Filename: rro6e4.all

Channels: Ch1 Ch2 Ch3 Ch4 Ch5 Ch6 Ch7

Plotted: 15:18 Mar 08, 2001

< EMI - ElectroMagnetic Instruments 


\section{Station 6}

TIPPER STRIKE

Rio Rancho - Albuquerque, NM

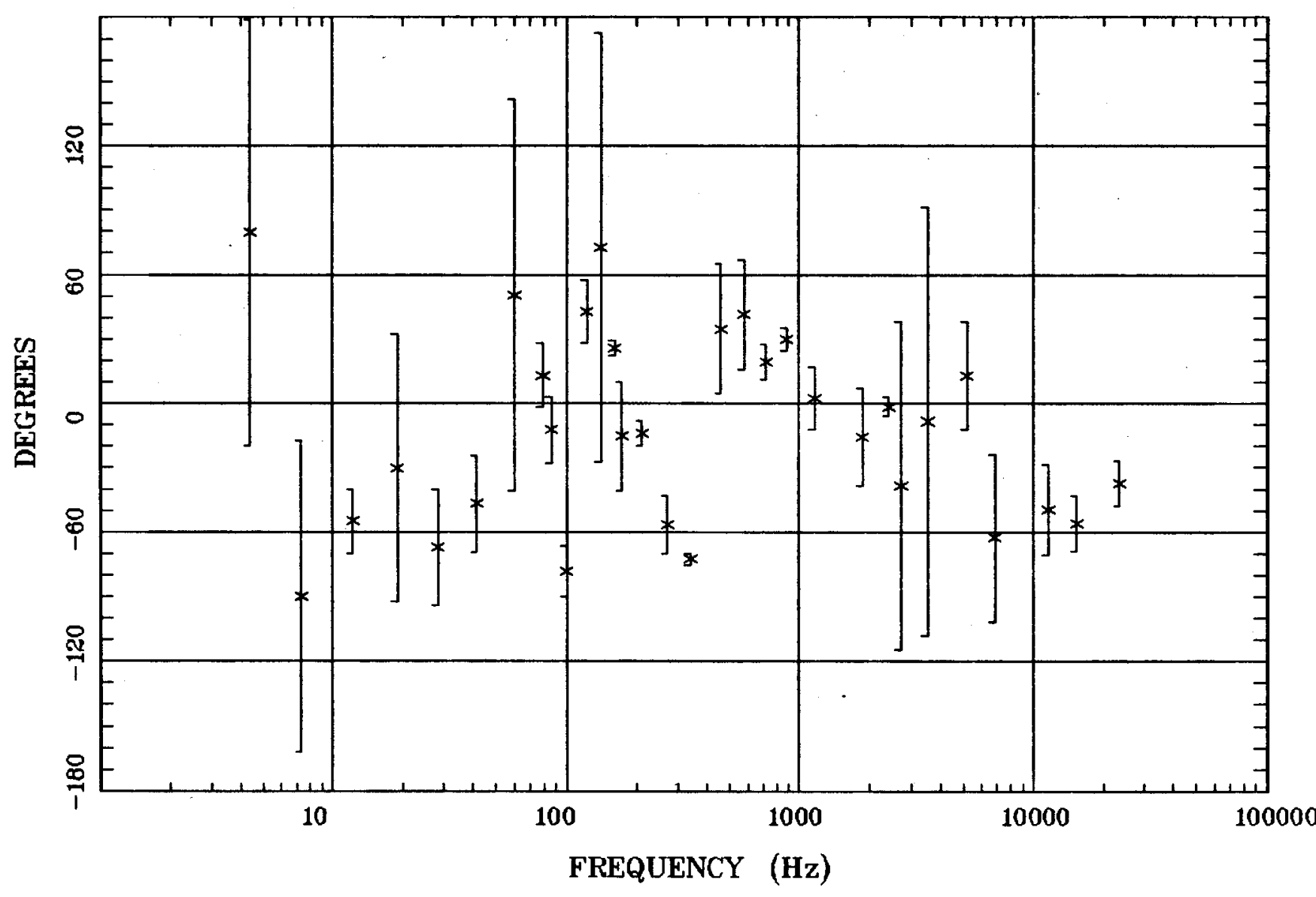

Client:

Remote: Local E

Acquired: 16:1 May 22, 1997

Survey Co:USGS GD-MRP Denver
Rotation:

Filename: rr06e4.all

Channels: Ch1 Ch2 Ch3 Ch4 Ch5 Ch6 Ch7

Platted: 15:18 Mar 08, 2001

< EMI - ElectroMagnetic Instruments 


\section{Station 6}

HzHx.x Coh HzHy.o Rio Rancho - Albuquerque, NM

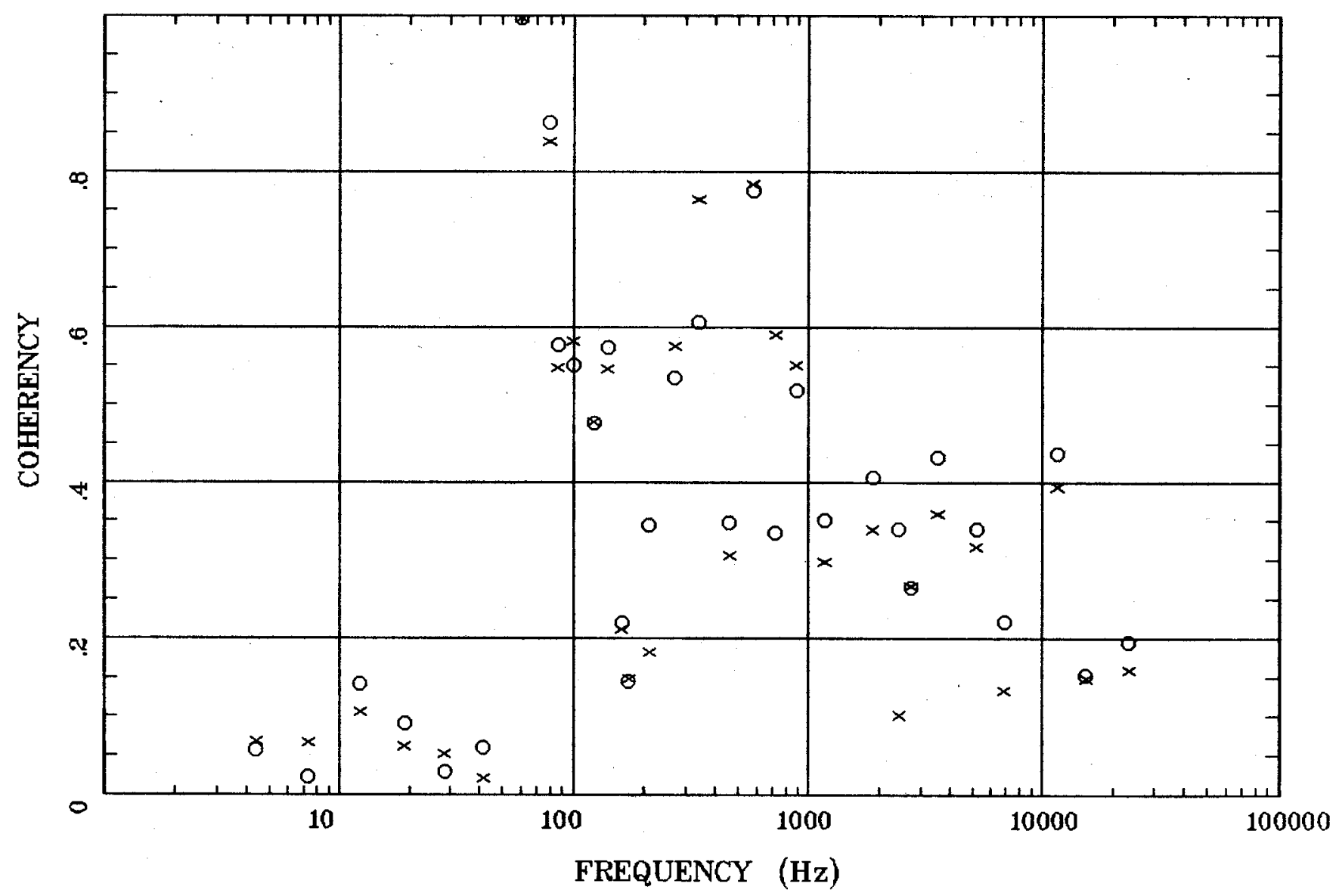

Client:

Remote: Local E

Acquired: 16:1 May 22, 1997

Survey Co:USGS GD-MRP Denver
Rotation:

Filename: rr06e4.all

Channels: Ch1 Ch2 Ch3 Ch4 Ch5 Ch6 Ch7

Plotted: 15:18 Mar 08, 2001

$<$ EMI - ElectroMagnetic Instruments 


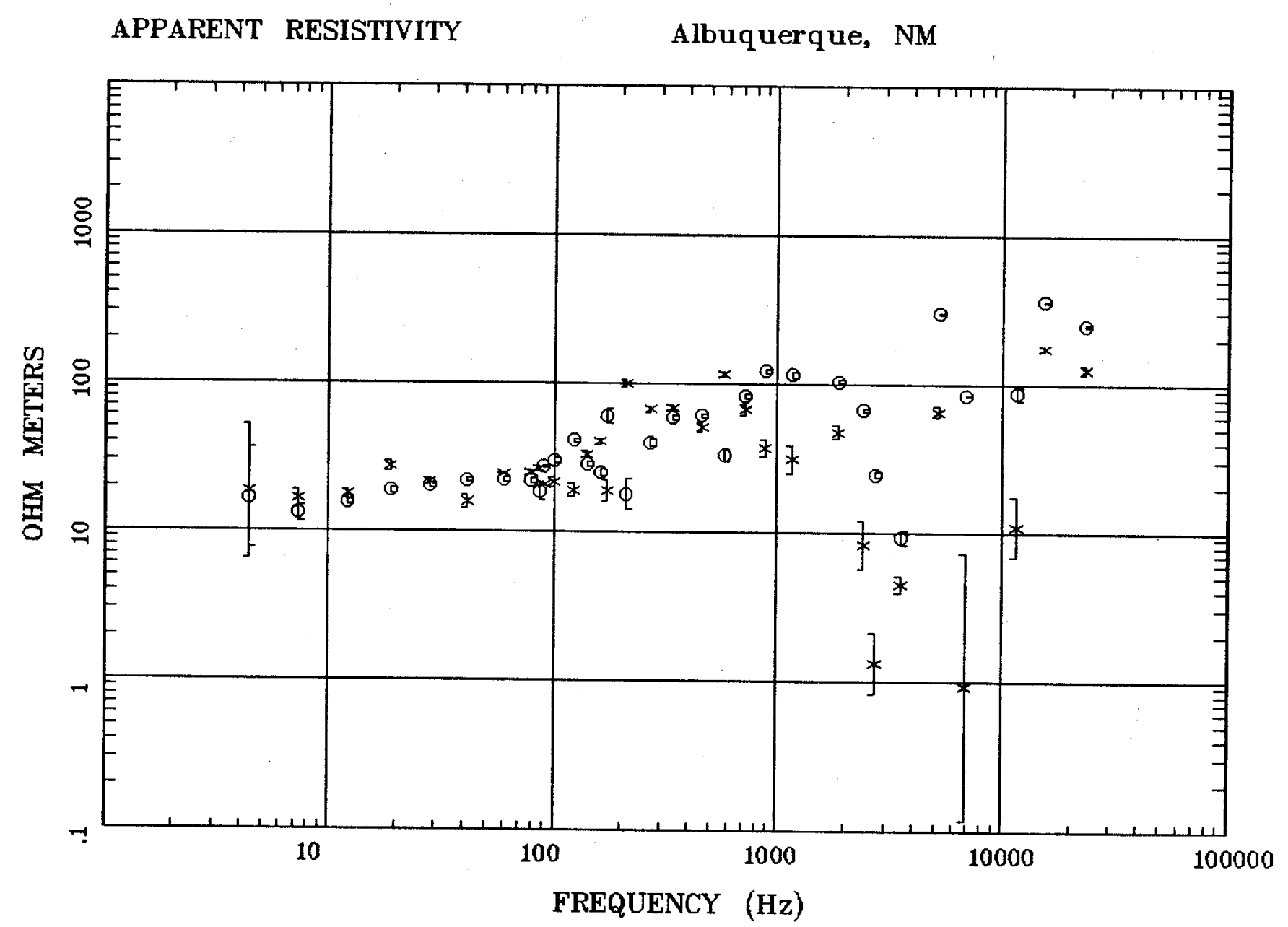

Rotation:

Client: City of Albuquerque

Filename: rr12all.avg Remote: $n$

Acquired: 08:1 May 08, 1998 Survey Co:USGS

Channels: Ch1 Ch2 Ch3 Ch4 Ch5 Ch8 Ch9

Plotted: 15:20 Mar 08, 2001

< EMI - ElectroMagnetic Instruments > 


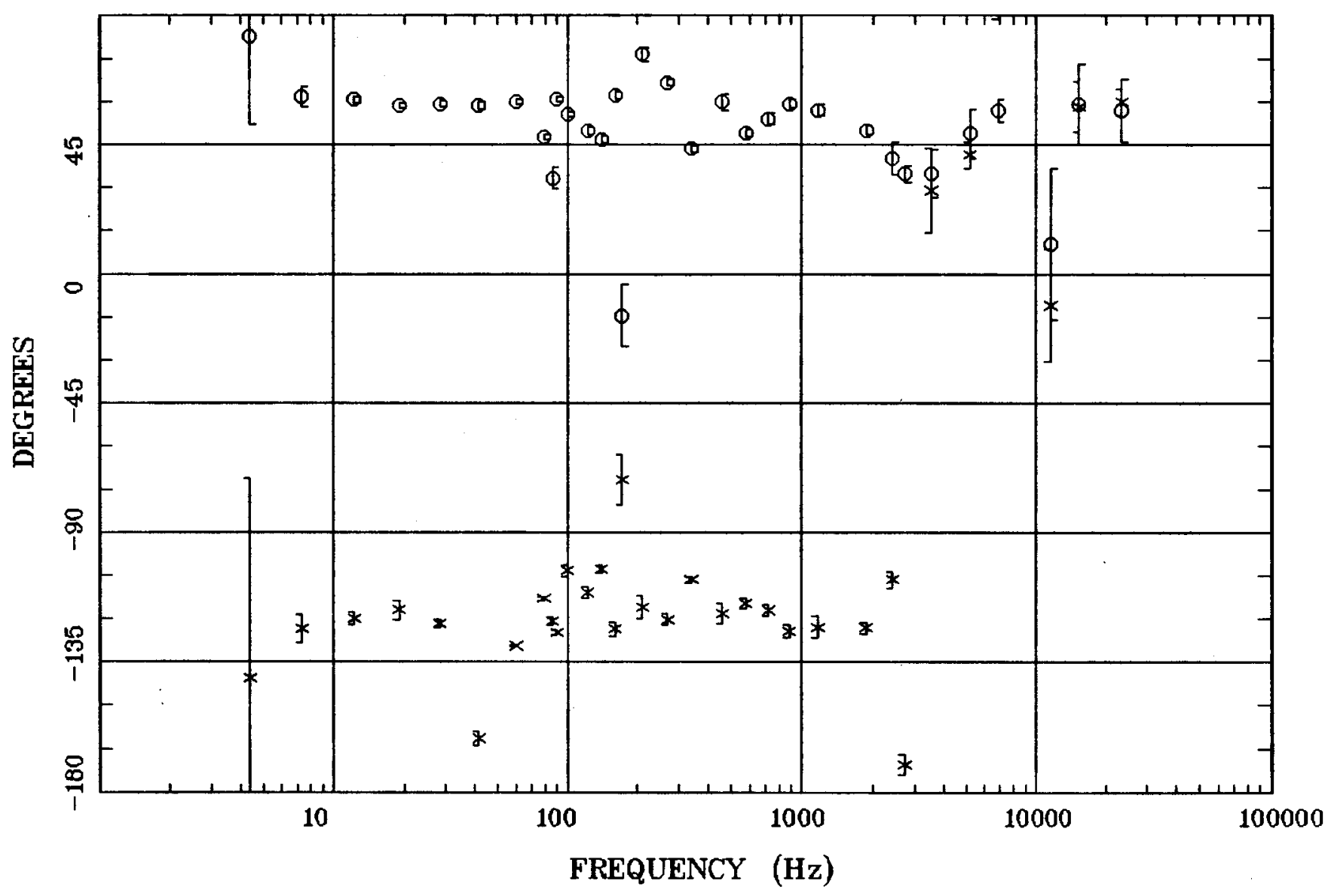

Client: City of Albuquerque Remote: $\mathbf{n}$ Acquired: 08:1 May 08, 1998 Survey Co:USGS
Rotation:

Filename: rr12all.avg

Channels: Ch1 Ch2 Ch3 Ch4 Ch5 Ch8 Ch9 Plotted: 15:20 Mar 08, 2001

< EMI - ElectroMagnetic Instruments 


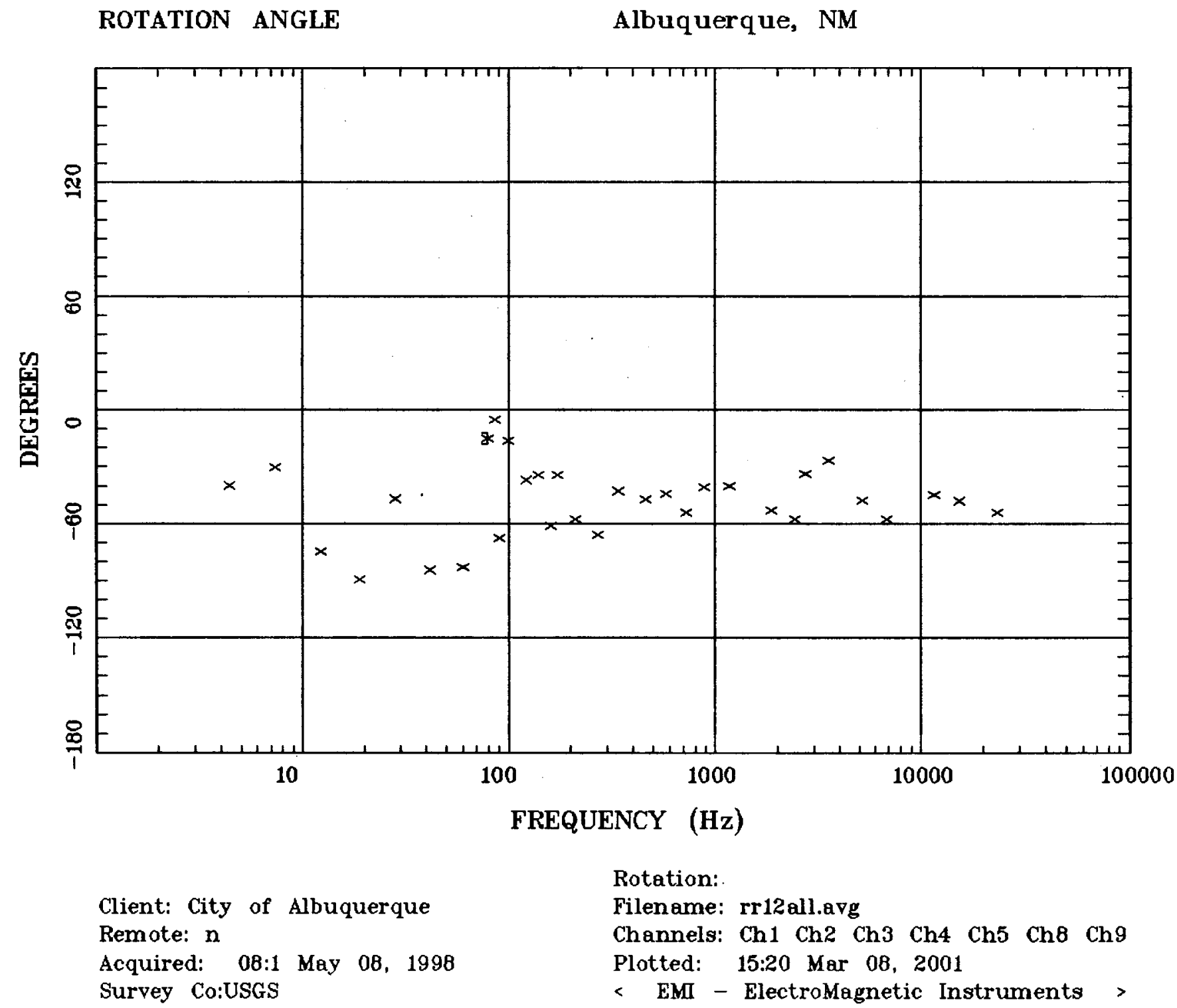




\section{Station 12}

Albuquerque, NM

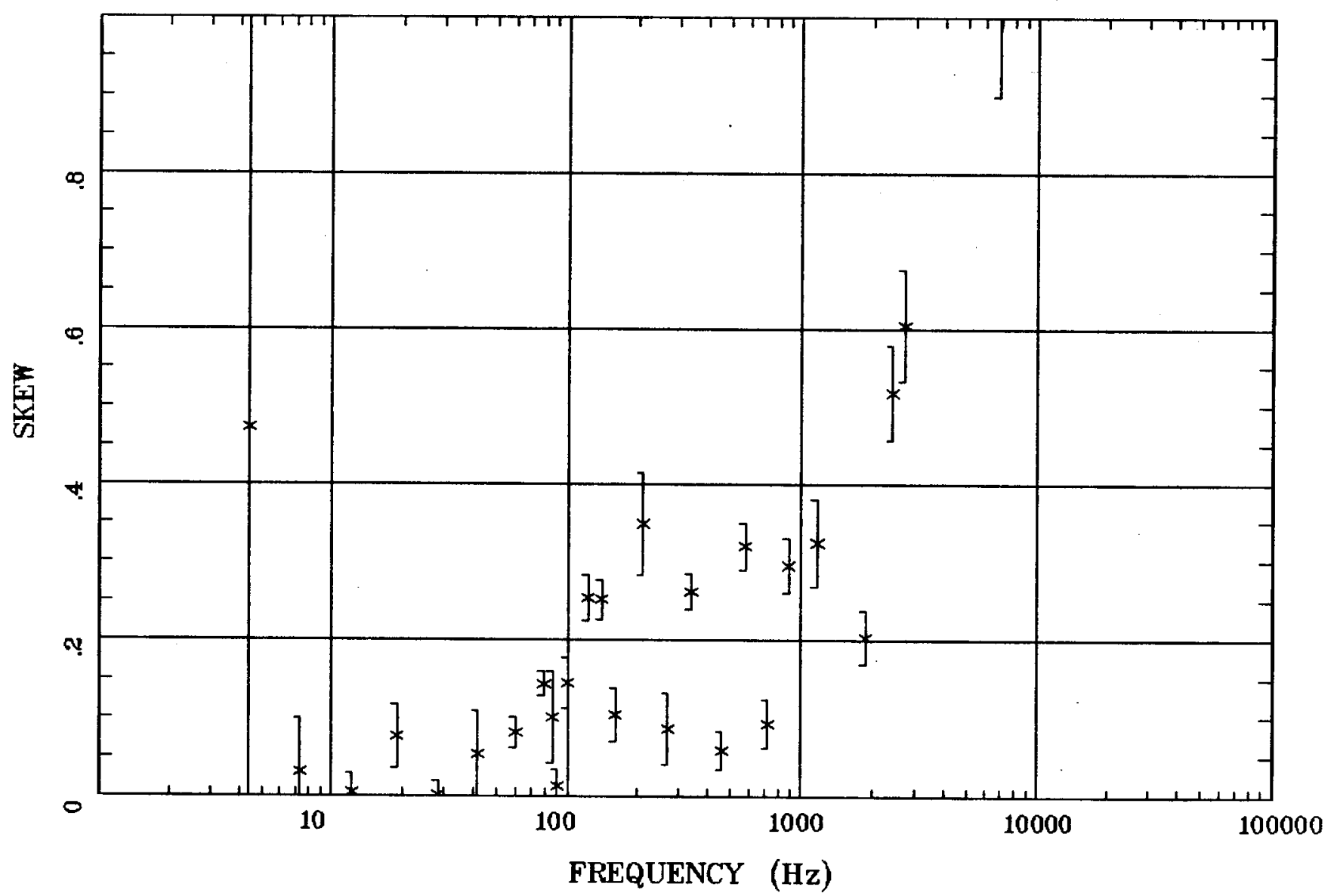

Client: City of Albuquerque Remote: $n$

Acquired: 08:1 May 08, 1998

Survey Co:USGS
Rotation:

Filename: rr12all.avg

Channels: Ch1 Ch2 Ch3 Ch4 Ch5 Ch8 Ch9

Plotted: 15:20 Mar 08, 2001

$<$ EMI - ElectroMagnetic Instruments 


\section{E MULT Coh.}

Albuquerque, NM

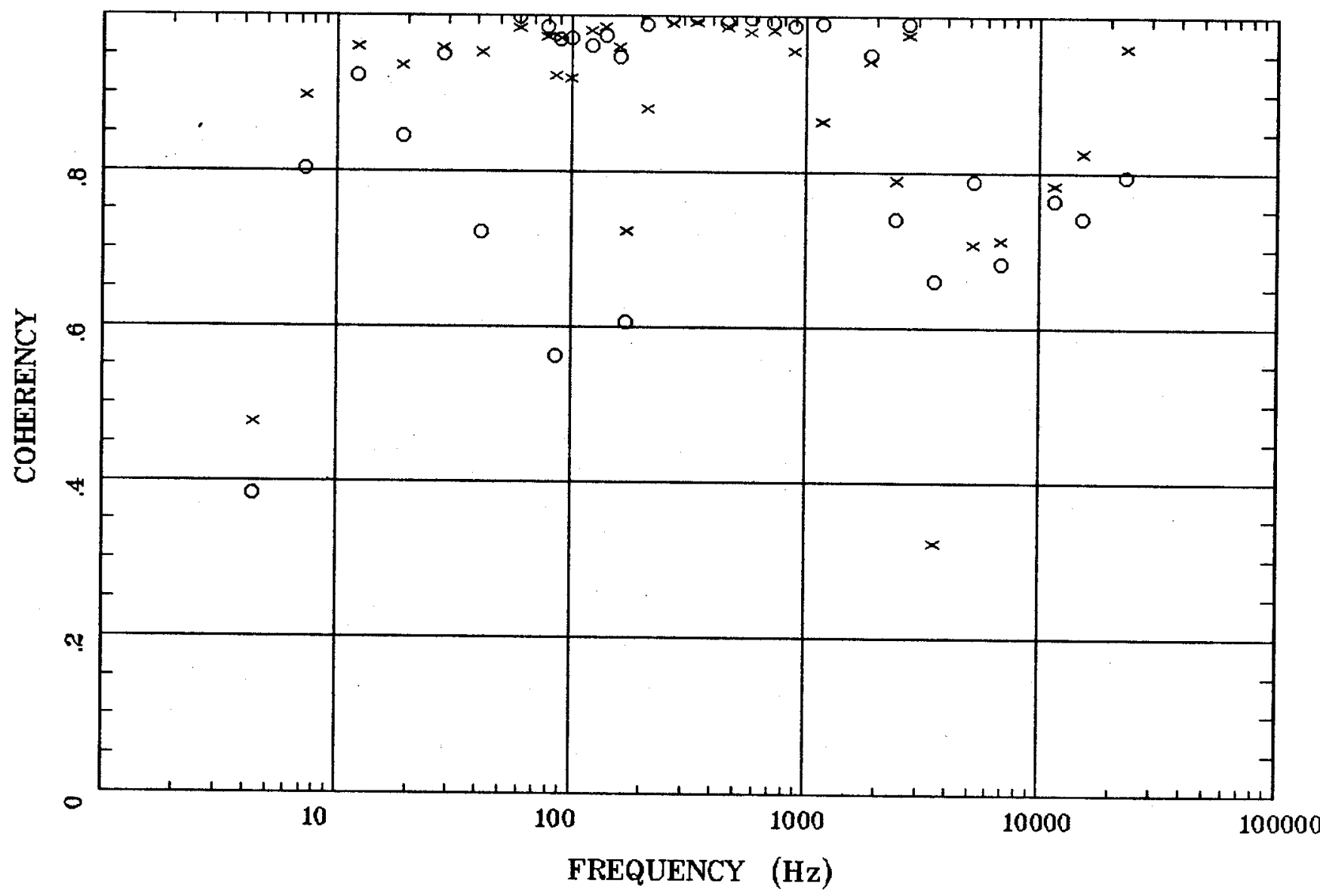

Client: City of Albuquerque Remote: $\mathbf{n}$ Acquired: 08:1 May 08, 1998 Survey Co:USGS
Rotation:

Filename: rr12all.avg

Channels: Ch1 Ch2 Ch3 Ch4 Ch5 Ch8 Ch9 Plotted: 15:20 Mar 08, 2001

< EMI - ElectroMagnetic Instruments > 


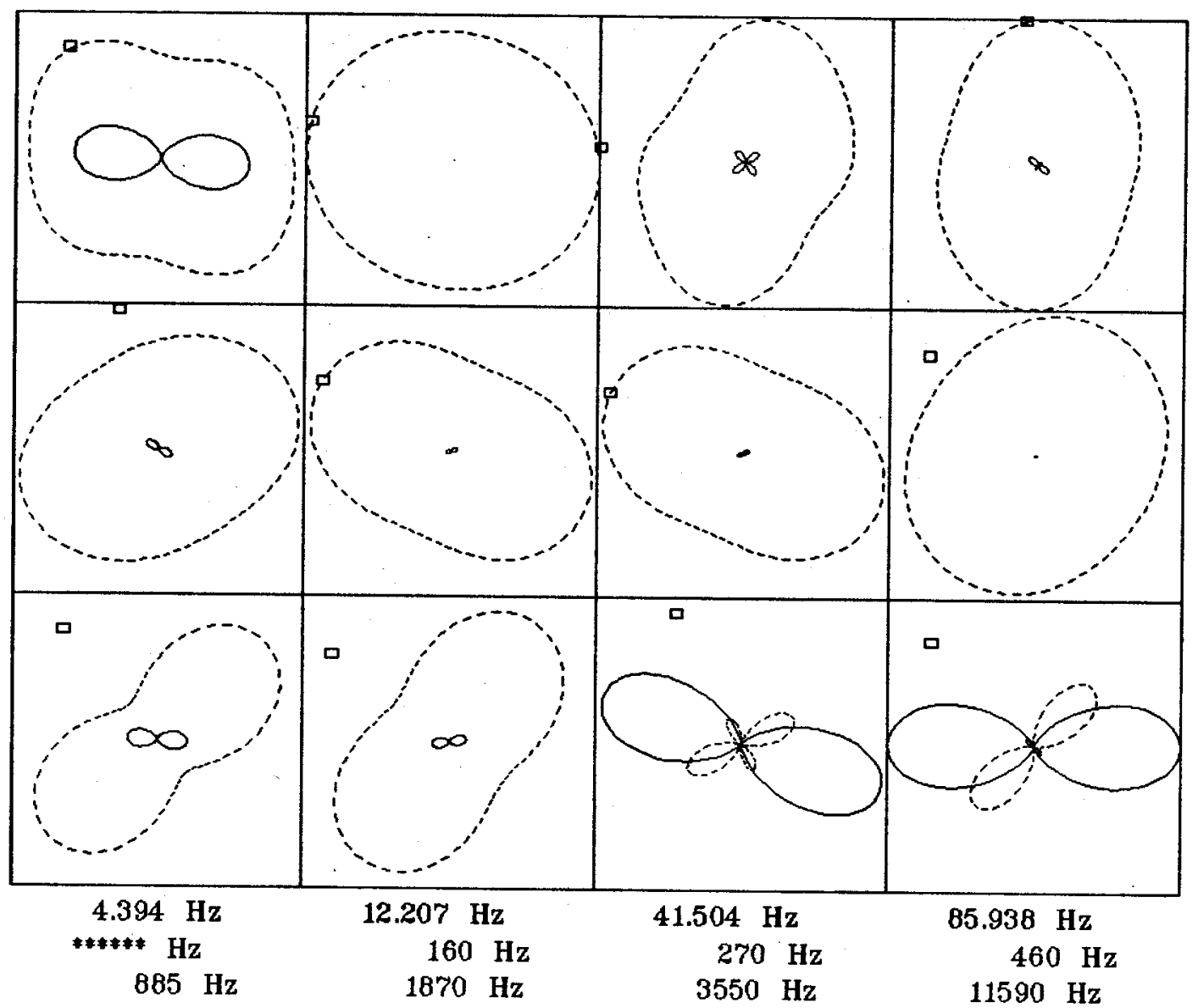

Client: City of Albuquerque Remote: $\mathbf{n}$

Acquired: 08:1 May 08, 1998 Survey Co:USGS
Rotation:

Filename: rr12all.avg

Channels: Ch1 Ch2 Ch3 Ch4 Ch5 Ch8 Ch9

Plotted: 15:20 Mar 08, 2001

$<$ EMI - ElectroMagnetic Instruments 


\section{Station 12}

\section{TIPPER MAGNITUDE}

Albuquerque, NM

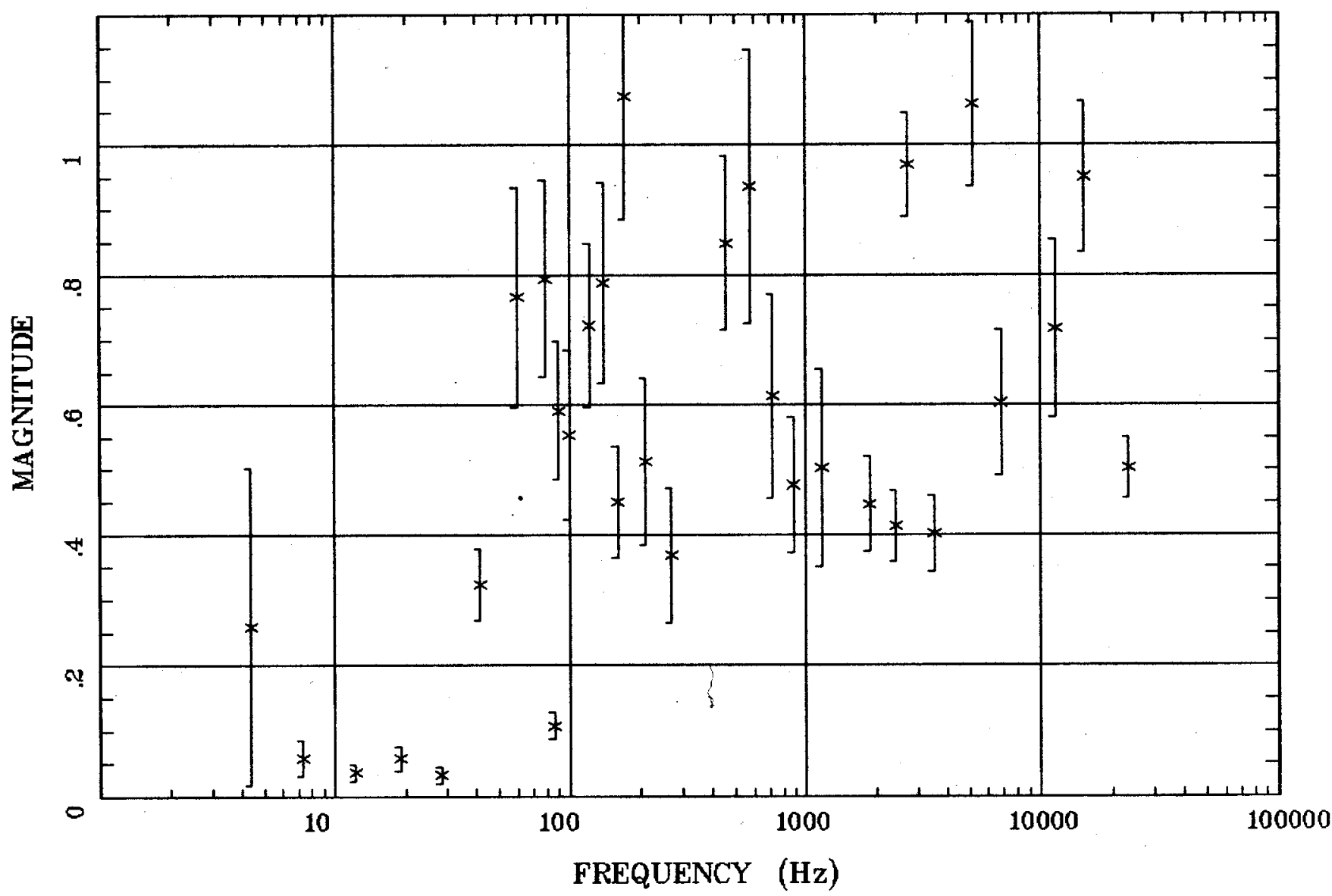

Client: City of Albuquerque Remote: $\mathrm{n}$

Acquired: 08:1 May 08, 1998 Survey Co:USGS
Rotation:

Filename: rr12all.avg

Channels: Ch1 Ch2 Ch3 Ch4 Ch5 Ch8 Ch9 Plotted: 15:20 Mar 08, 2001

< EMI - ElectroMagnetic Instruments > 


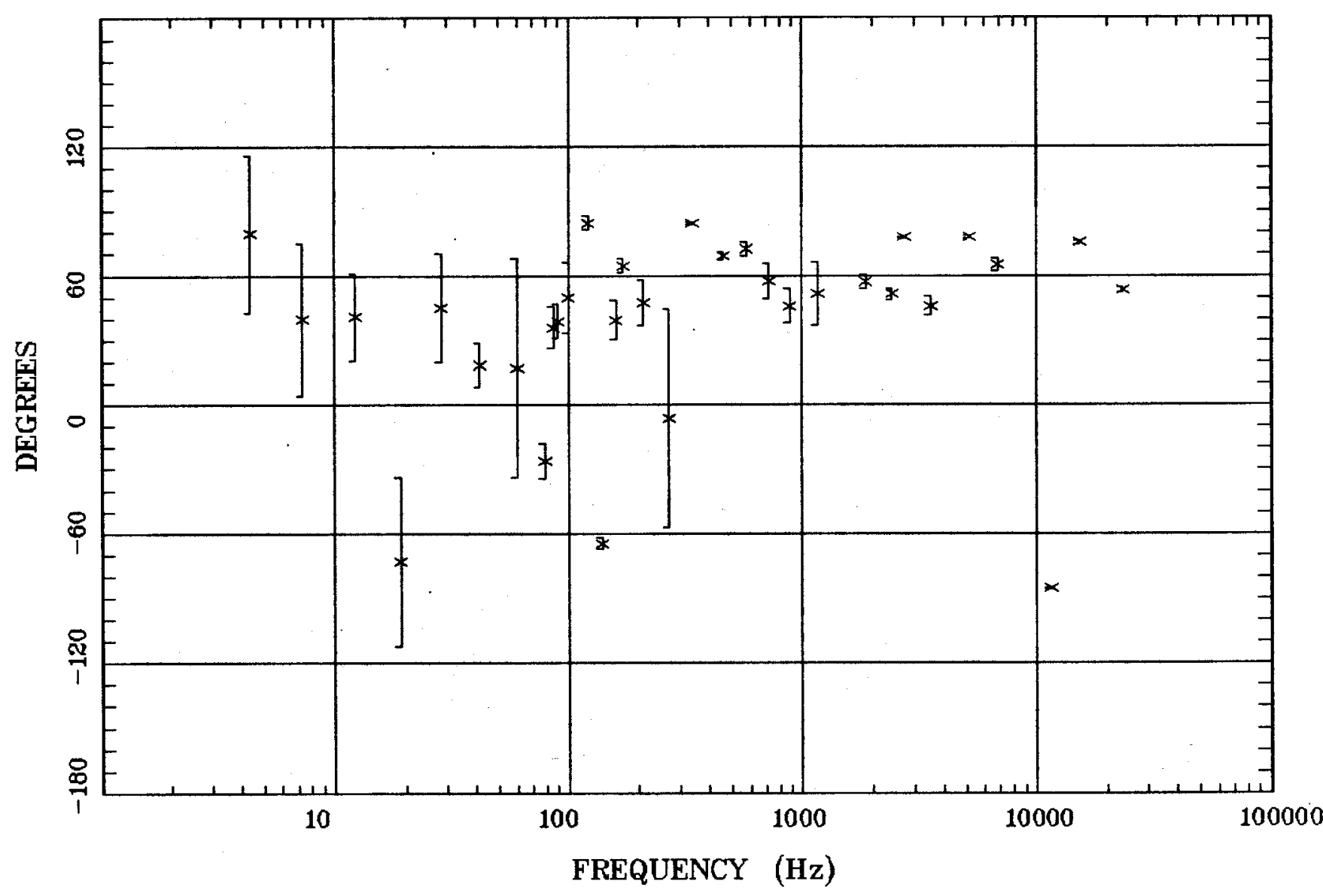

Client: City of Albuquerque Remote: $\mathbf{n}$

Acquired: 08:1 May 08, 1998 Survey Ca:USGS
Rotation:

Filename: rr12all.avg

Channels: Ch1 Ch2 Ch3 Ch4 Ch5 Ch8 Ch9

Plotted: 15:20 Mar 08, 2001

< EMI - ElectroMagnetic Instruments > 
Station 12

HzHx.x Coh HzHy.o

Albuquerque, NM

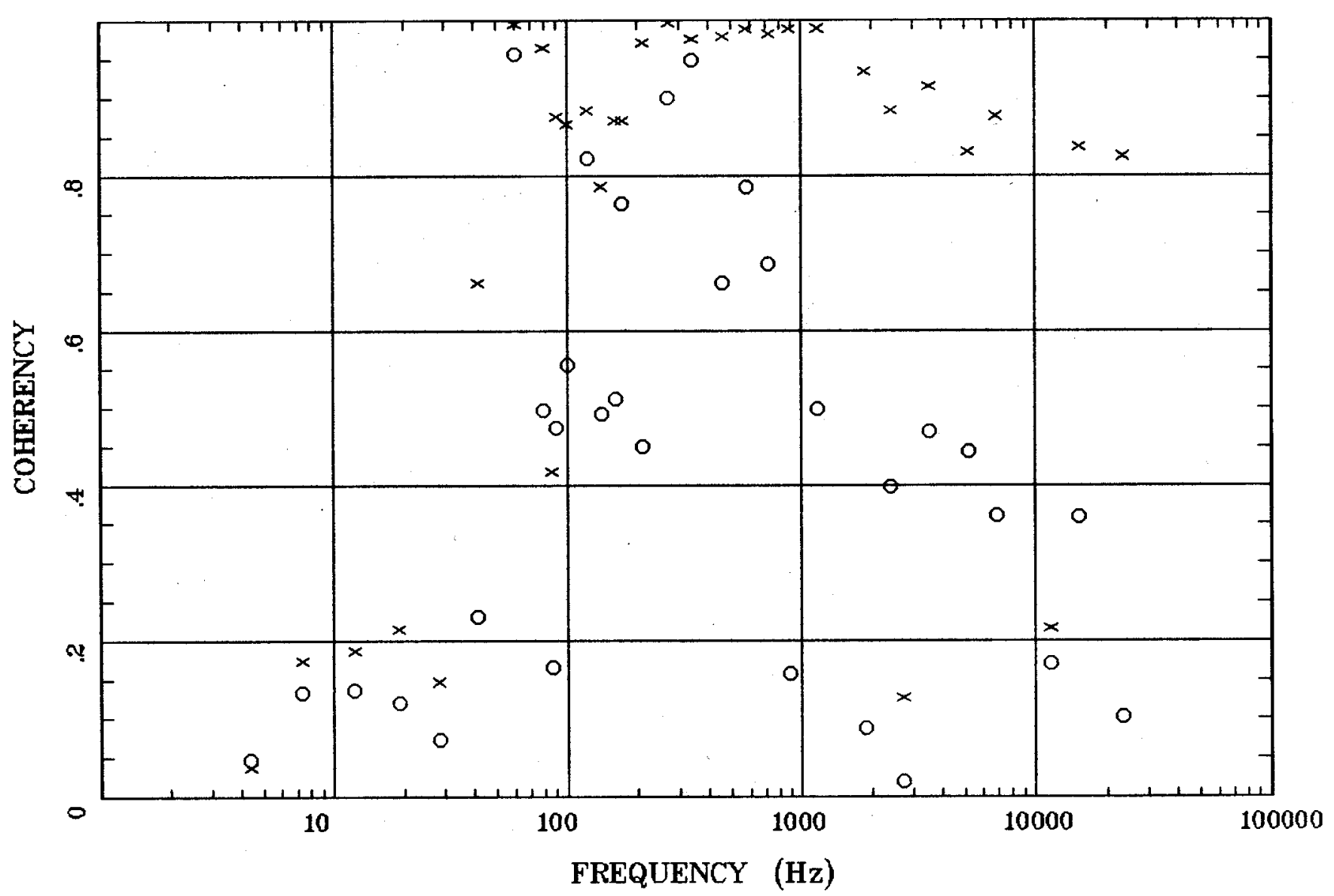

Client: City of Albuquerque Remote: $\mathbf{n}$

Acquired: 08:1 May 08, 1998 Survey Co:USGS
Rotation:

Filename: rr12all.avg

Channels: Ch1 Ch2 Ch3 Ch4 Ch5 Ch8 Ch9

Plotted: 15:20 Mar 08, 2001

< EMI - ElectroMagnetic Instruments > 


\section{Station 10}

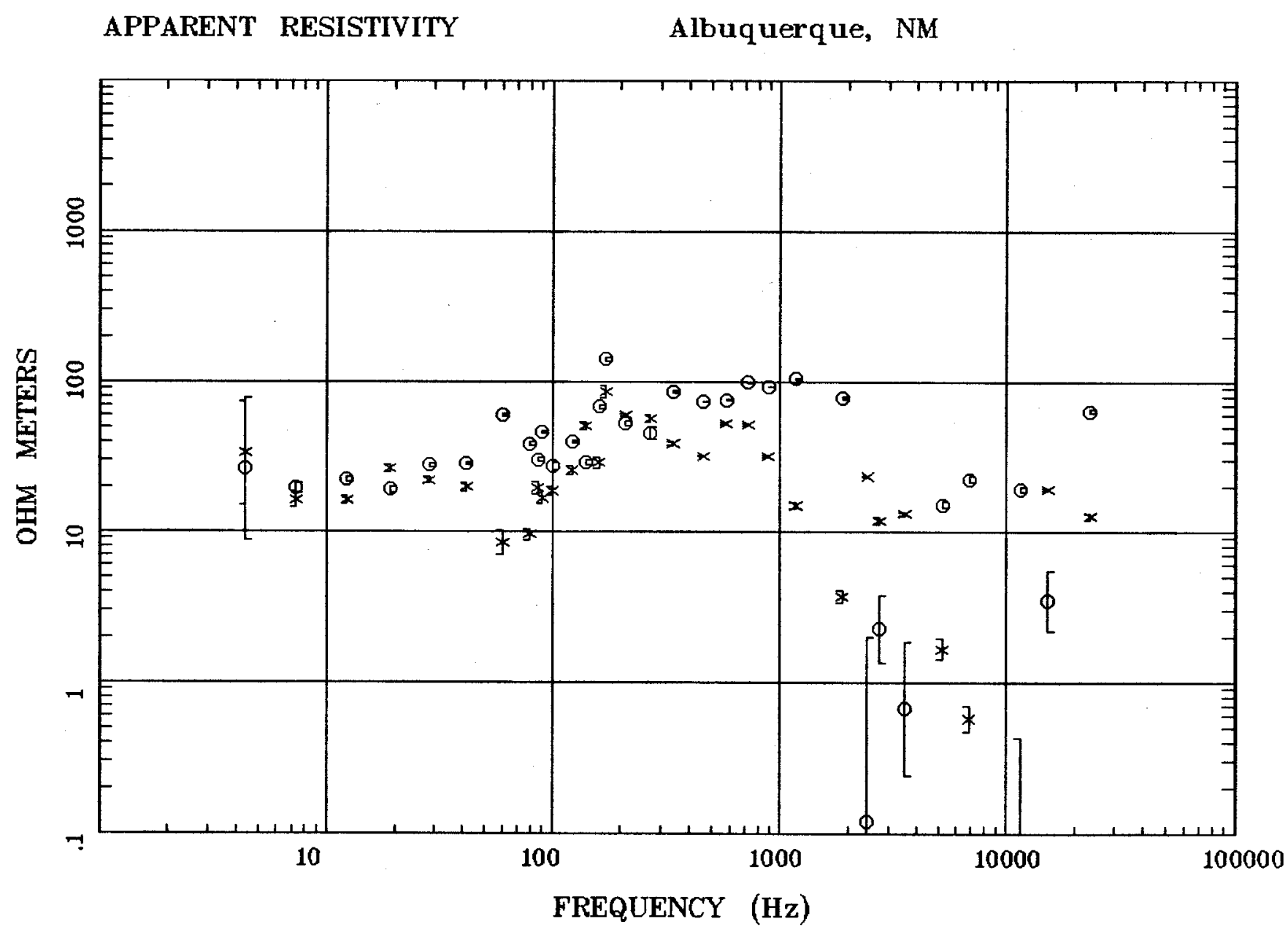

Client: City of Albuquerque

Remote: $\mathbf{n}$

Acquired: 09:2 May 07, 1998 Survey Co:USGS
Rotation:

Filename: rr10all.avg

Channels: Ch1 Ch2 Ch3 Ch4 Ch5 Ch8 Ch9 Plotted: 15:36 Mar 08, 2001

< EMI - ElectroMagnetic Instruments 
IMPEDANCE PHASE

Albuquerque, NM

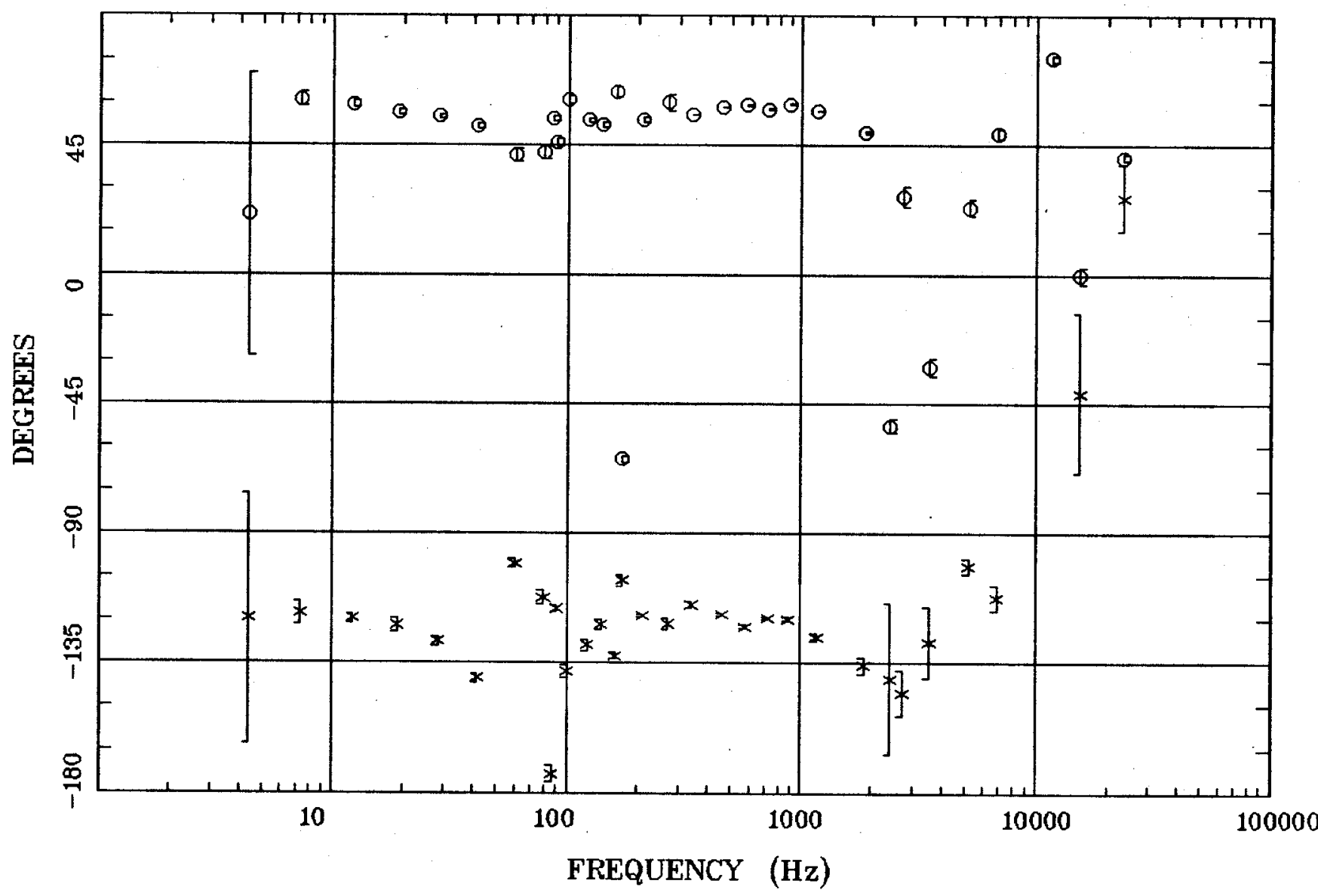

Client: City of Albuquerque

Remote: $\mathbf{n}$

Acquired: 09:2 May 07, 1998

Survey Co:USGS
Rotation:

Filename: rr10all.avg

Channels: Ch1 Ch2 Ch3 Ch4 Ch5 Ch8 Ch9

Plotted: 15:36 Mar 08, 2001

$<$ EMI - ElectroMagnetic Instruments 
ROTATION ANGLE Albuquerque, NM

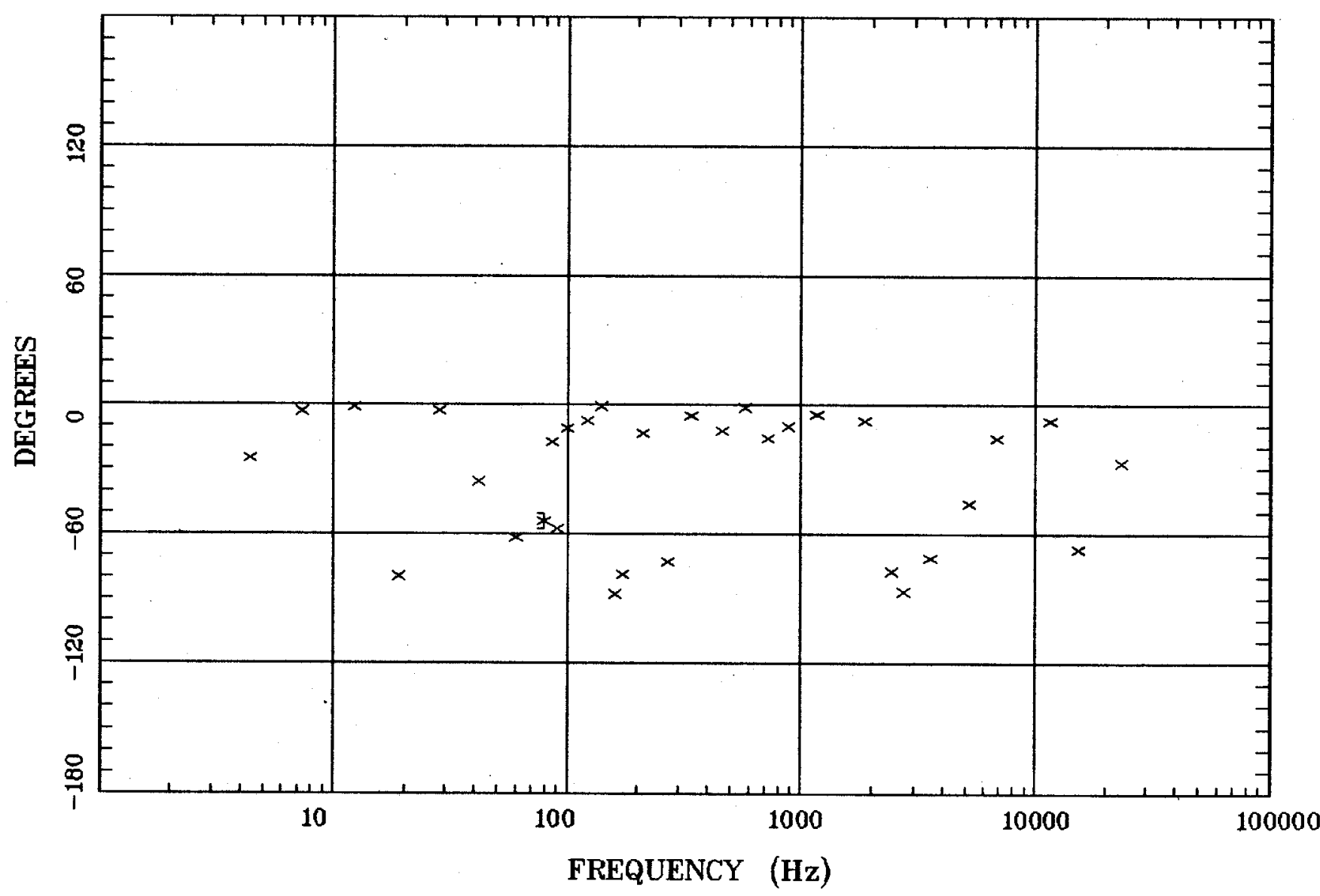

Client: City of Albuquerque

Remote: $\mathbf{n}$

Acquired: 09:2 May 07, 1998

Survey Co:USGS
Rotation:

Filename: rr10all.avg

Channels: Ch1 Ch2 Ch3 Ch4 Ch5 Ch8 Ch9

Plotted: 15:36 Mar 08, 2001

< EMI - ElectroMagnetic Instruments > 
Station 10

IMPEDANCE SKEW

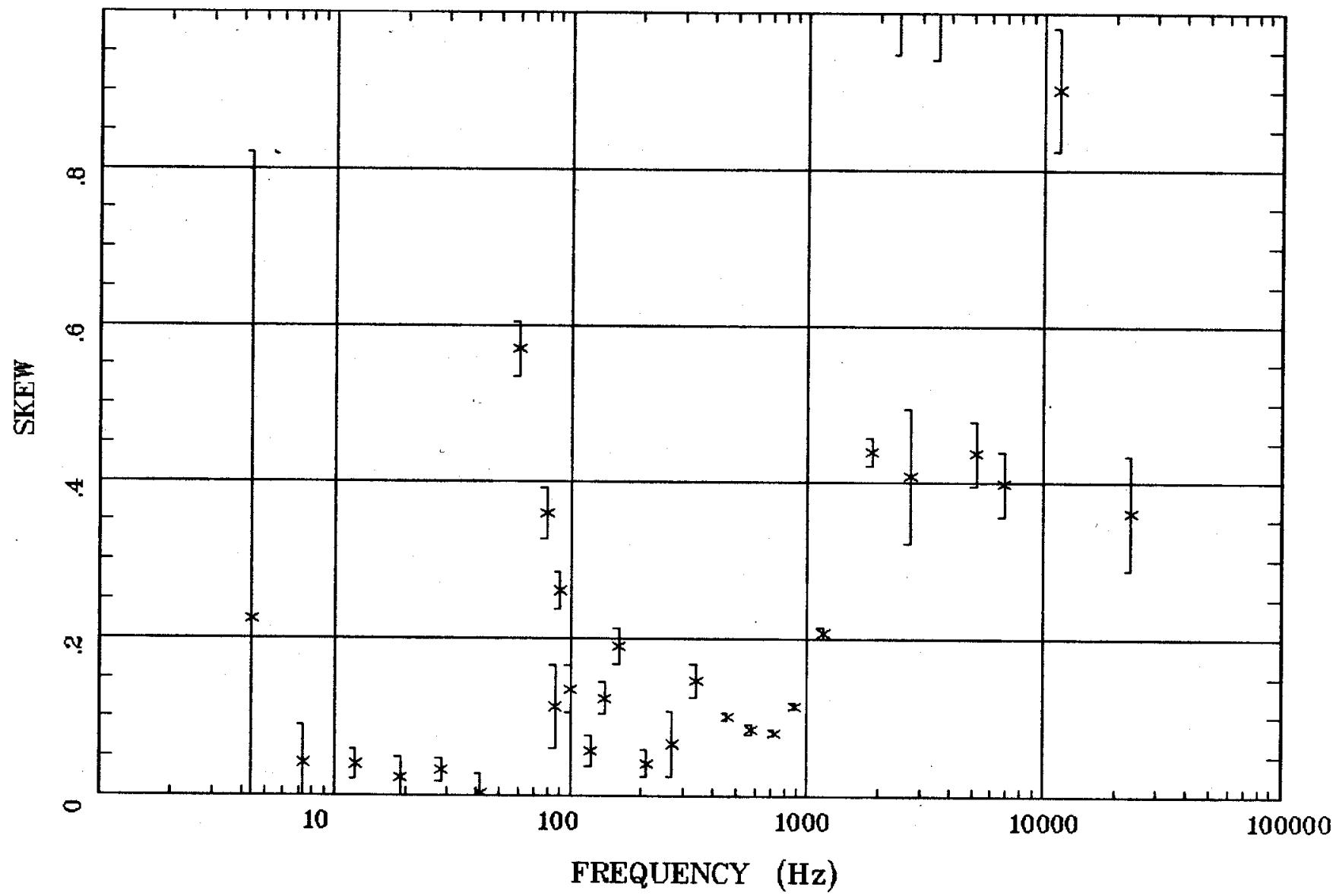

Client: City of Albuquerque Remote: $\mathbf{n}$

Acquired: 09:2 May 07, 1998 Survey Co:USGS
Rotation:

Filename: rr10all.avg

Channels: Ch1 Ch2 Ch3 Ch4 Ch5 Ch8 Ch9

Plotted: 15:36 Mar 08, 2001

$<$ EMI - ElectroMagnetic Instruments > 
E MULT Coh.

Albuquerque, NM

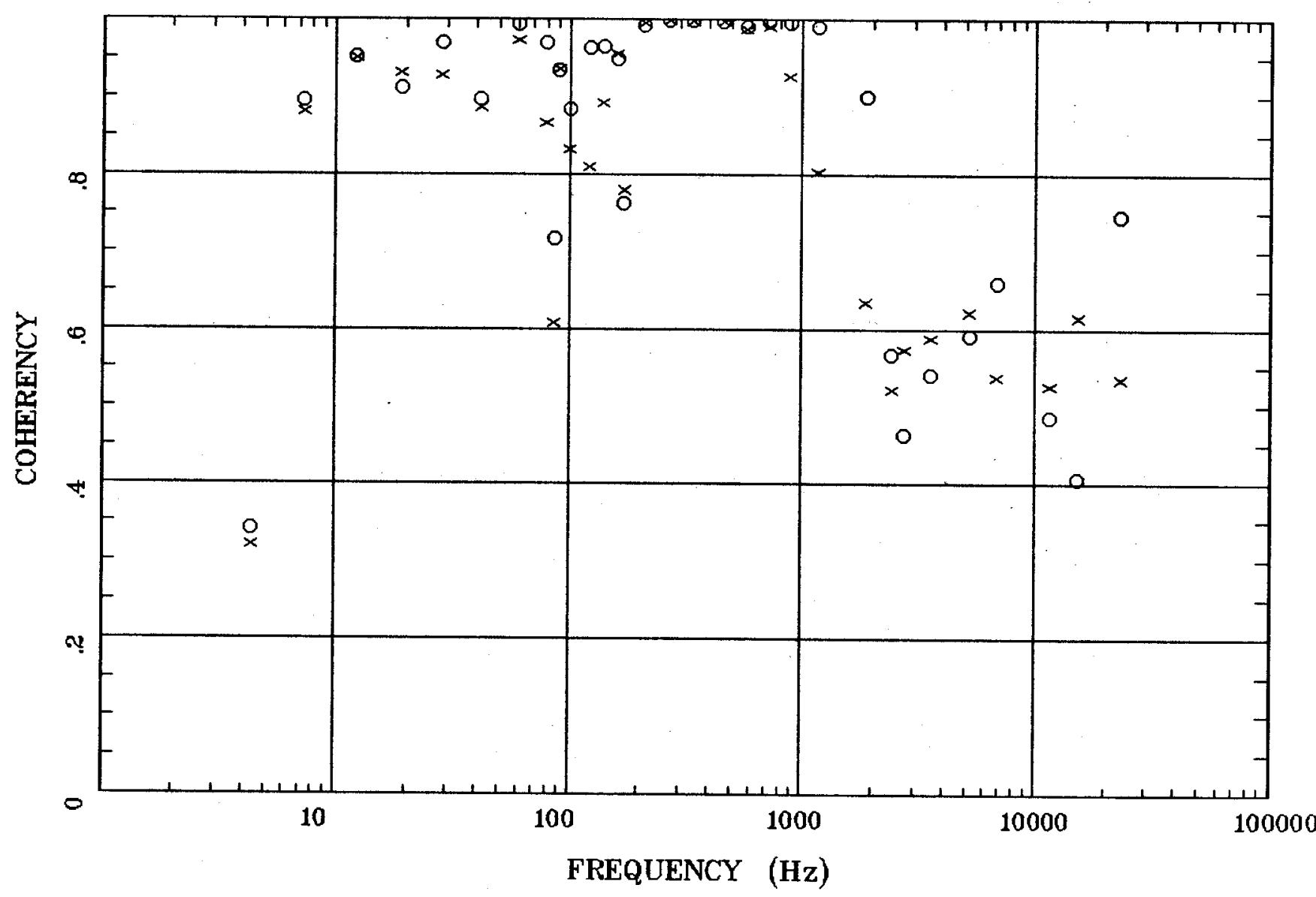

Client: City of Albuquerque

Remote: $\mathbf{n}$

Acquired: 09:2 May 07, 1998

Survey Co:USGS
Rotation:

Filename: rr10all.avg

Channels: Ch1 Ch2 Ch3 Ch4 Ch5 Ch8 Ch9

Plotted: 15:36 Mar 08, 2001

<EMI - ElectroMagnetic Instruments > 


\section{Station 10}

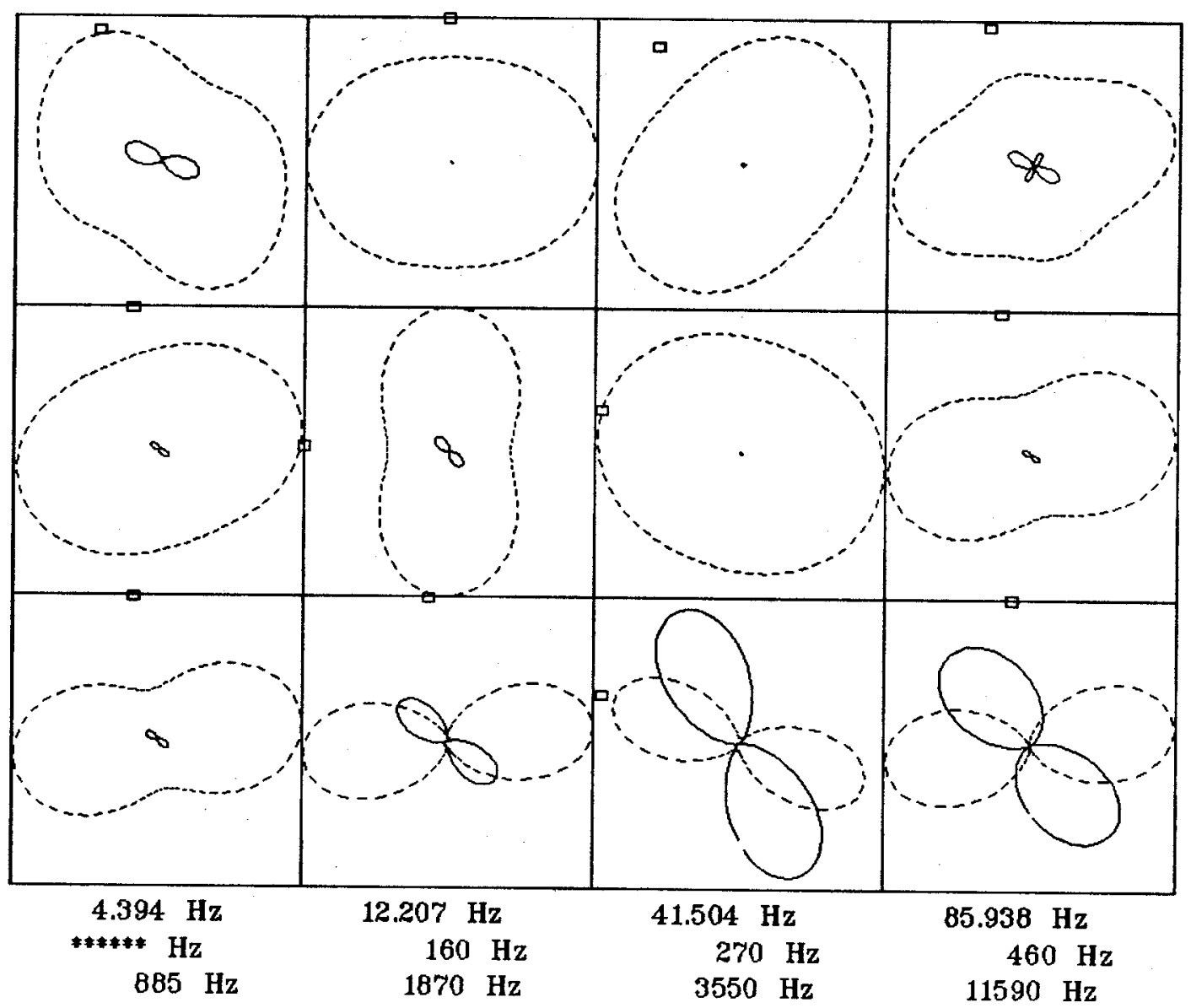

Client: City of Albuquerque

Rotation:

Remote: $n$

Filename: rr10all.avg

Channels: Ch1 Ch2 Ch3 Ch4 Ch5 Ch8 Ch9

Acquired: 09:2 May 07, 1998

Plotted: 15:36 Mar 08, 2001 
TIPPER MAGNITUDE

Albuquerque, NM

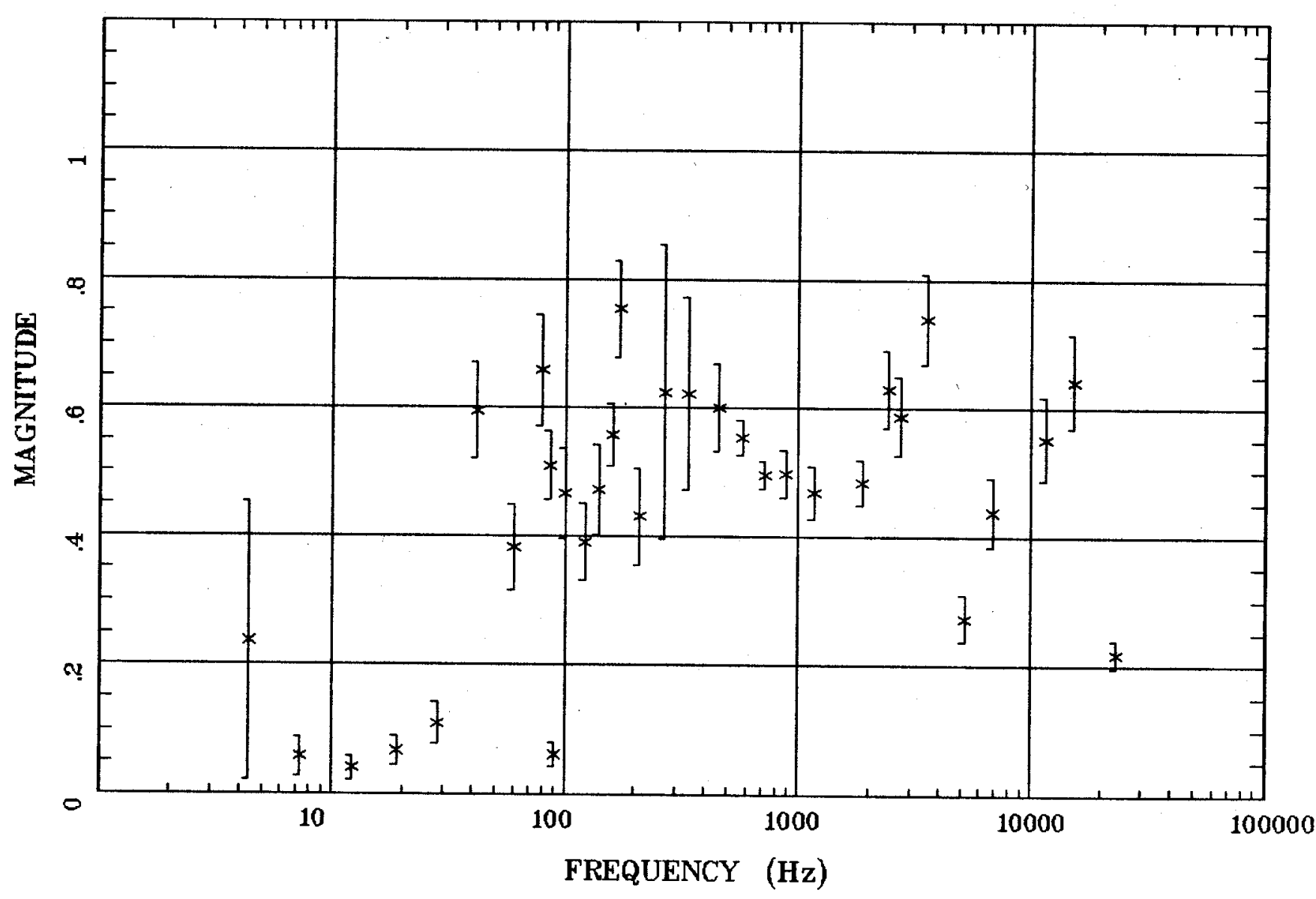

Client: City of Albuquerque Remote: $\mathbf{n}$

Acquired: 09:2 May 07, 1998 Survey Co:USGS
Rotation:

Filename: rr10all.avg

Channels: Ch1 Ch2 Ch3 Ch4 Ch5 Ch8 Ch9 Plotted: 15:36 Mar 08, 2001

< EMI - ElectroMagnetic Instruments > 
TIPPER STRIKE

Albuquerque, NM

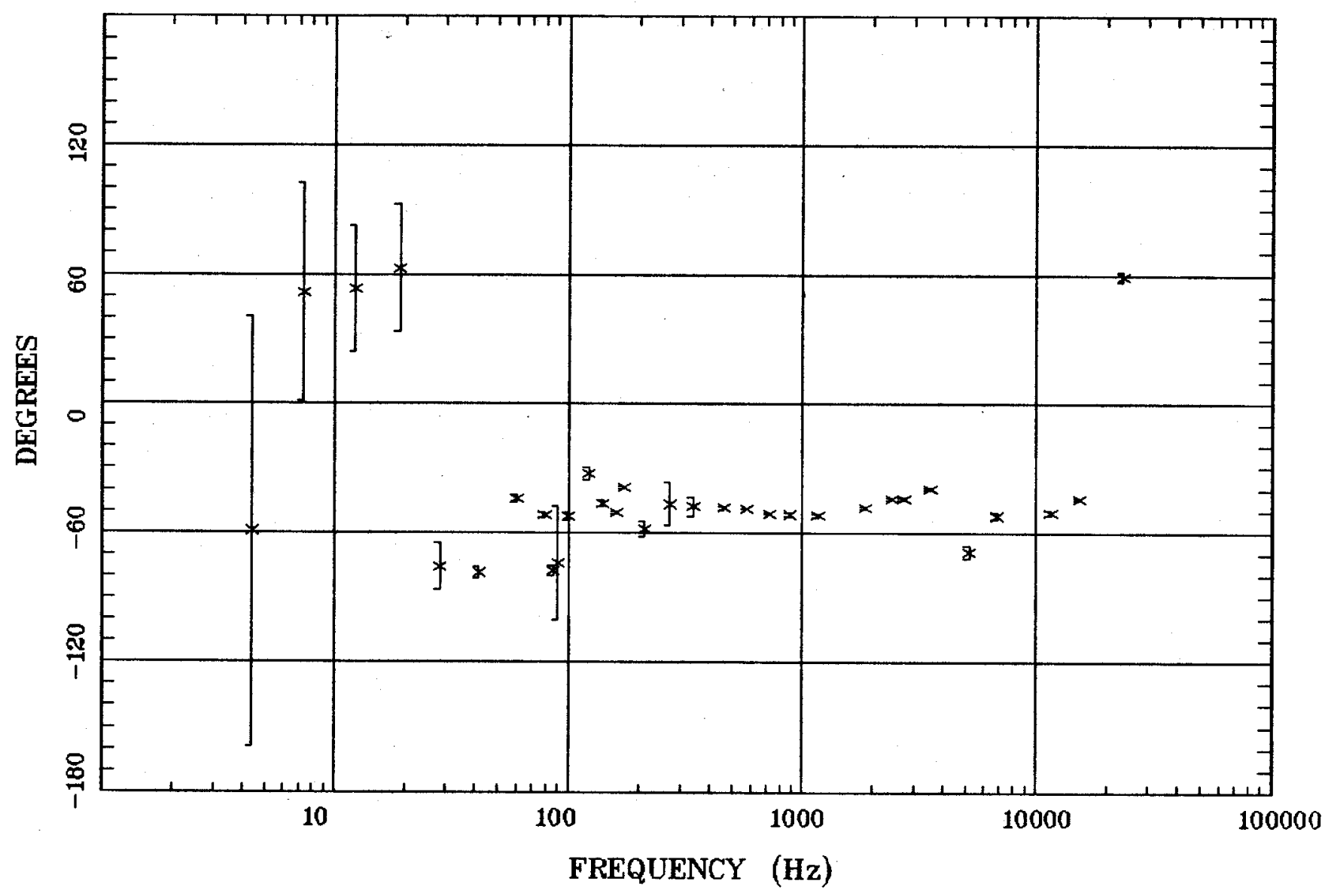

Client: City of Albuquerque Remote: $\mathbf{n}$

Acquired: 09:2 May 07, 1998 Survey Co:USGS
Rotation:

Filename: rr10all.avg

Channels: Ch1 Ch2 Ch3 Ch4 Ch5 Ch8 Ch9

Platted: 15:36 Mar 08, 2001

< EMI - ElectroMagnetic Instruments > 
HzHx.x Coh HzHy.o

Albuquerque, NM

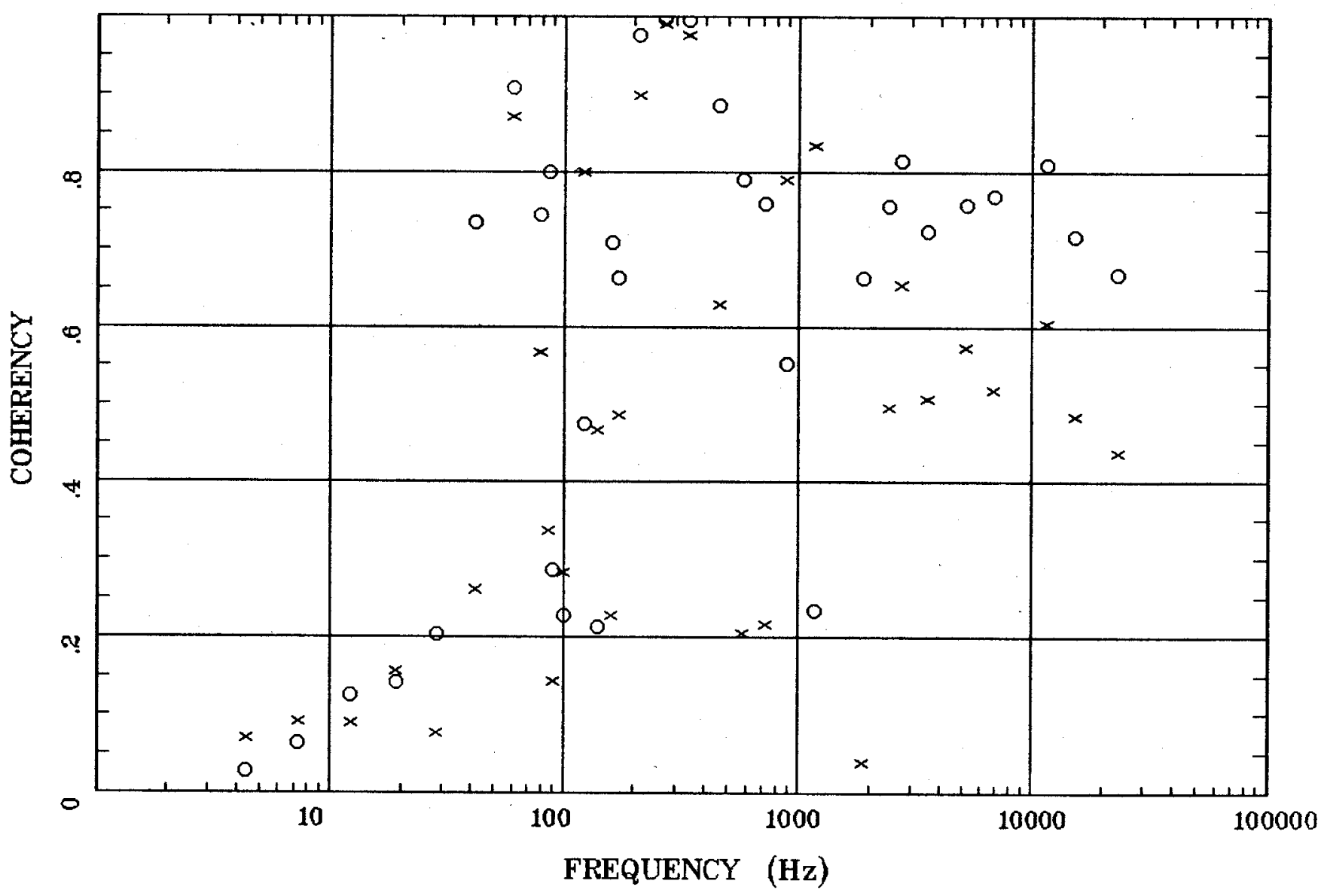

Client: City of Albuquerque Remote: $\mathbf{n}$

Acquired: 09:2 May 07, 1998 Survey Co:USGS
Rotation:

Filename: rr10all.avg

Channels: Ch1 Ch2 Ch3 Ch4 Ch5 Ch8 Ch9

Plotted: 15:36 Mar 08, 2001

< EMI - ElectroMagnetic Instruments > 


\section{Station 11}

APPARENT RESISTIVITY

Albuquerque, NM

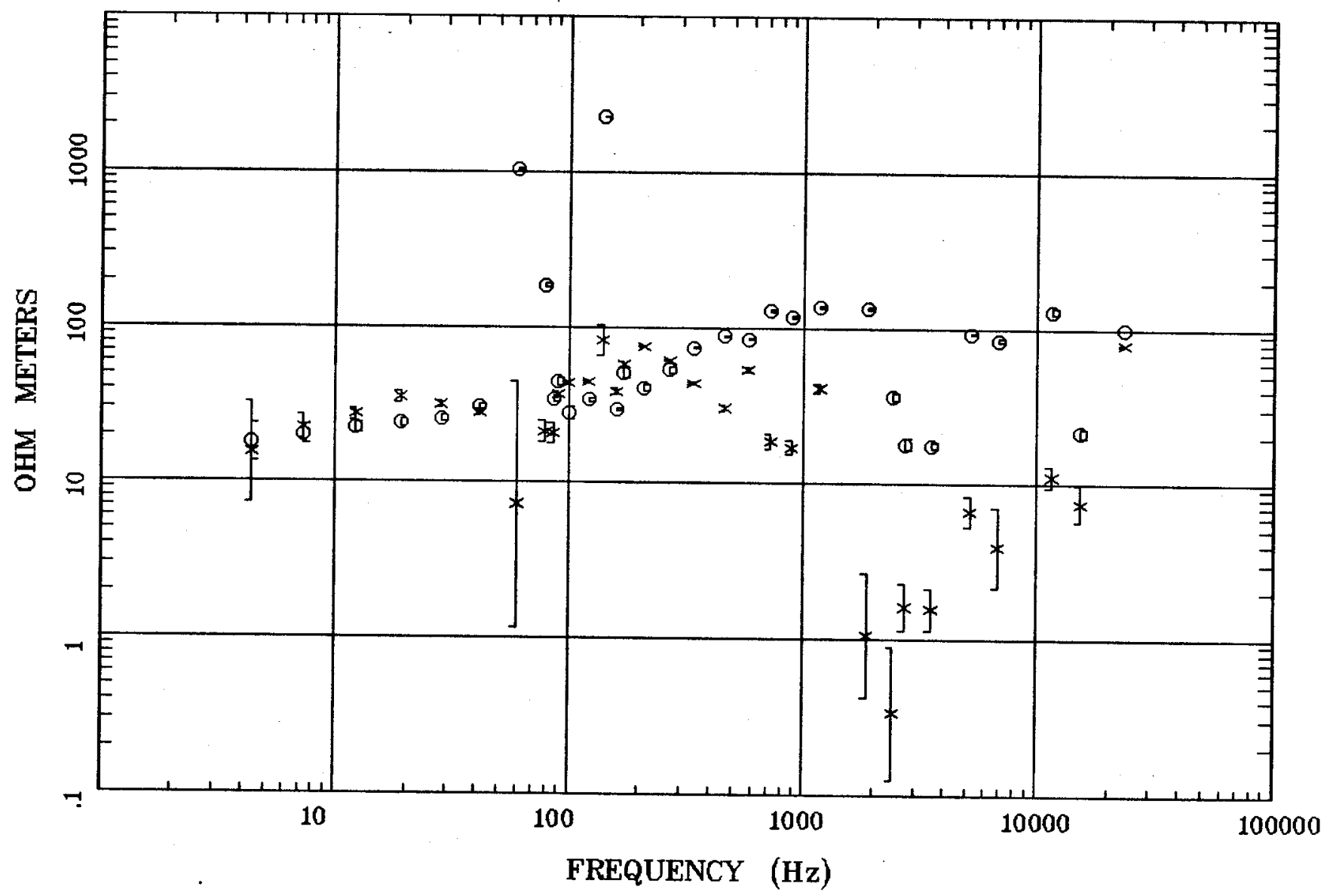

Client: City of Albuquerque Remote: $\mathbf{n}$

Acquired: 12:5 May 07, 1998 Survey Co:USGS
Rotation:

Filename: rr11all.avg

Channels: Ch1 Ch2 Ch3 Ch4 Ch5 Ch8 Ch9

Plotted: 15:37 Mar 08, 2001

$<$ EMI - ElectroMagnetic Instruments 
Station 11

IMPEDANCE PHASE

Albuquerque, NM

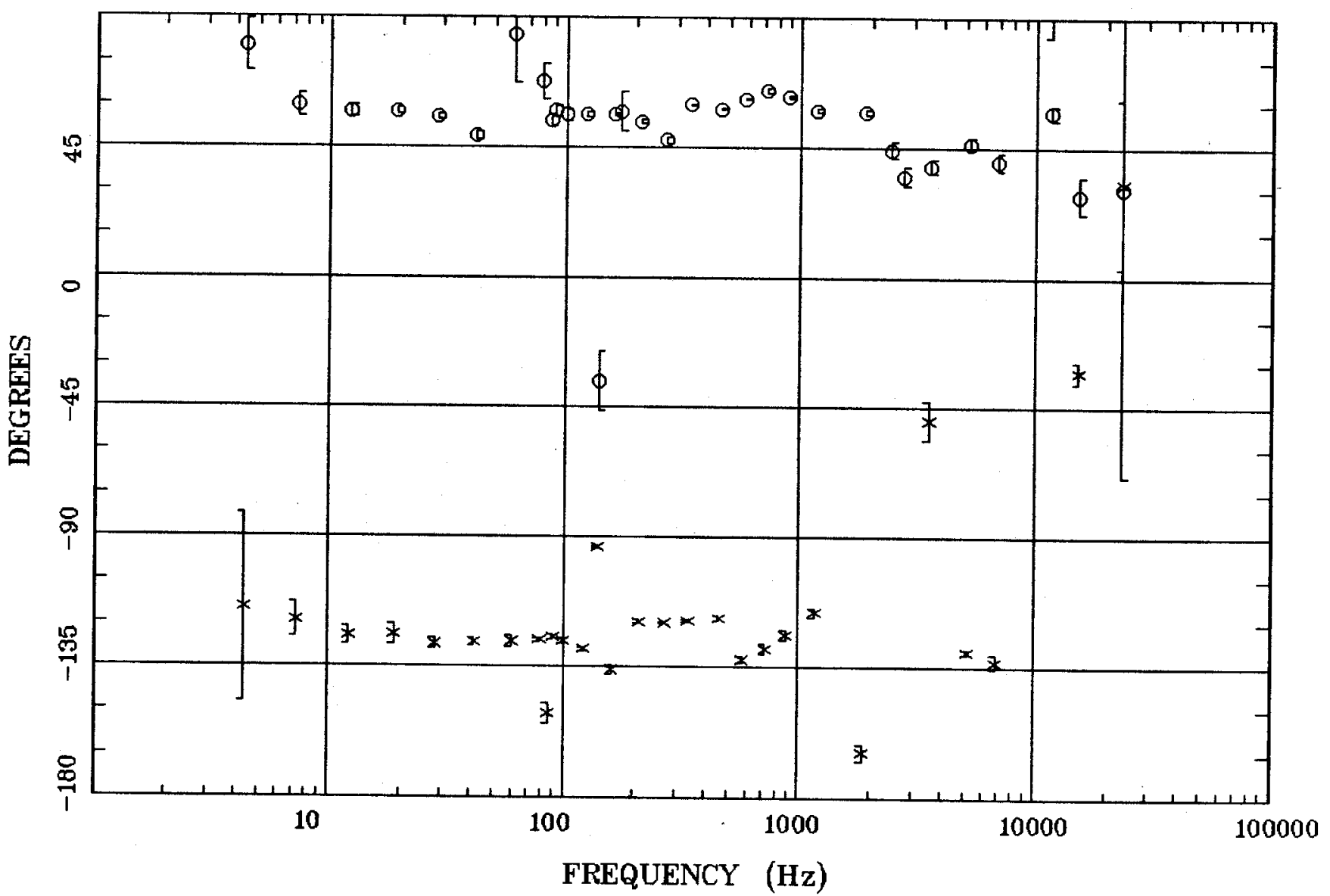

Client: City of Albuquerque Remote: $\mathbf{n}$

Acquired: 12:5 May 07, 1998 Survey Co:USGS
Rotation:

Filename: rrilall.avg

Channels: Ch1 Ch2 Ch3 Ch4 Ch5 Ch8 Ch9

Plotted: 15:37 Mar 08, 2001

$<$ EMI - ElectroMagnetic Instruments 


\section{Station 11}

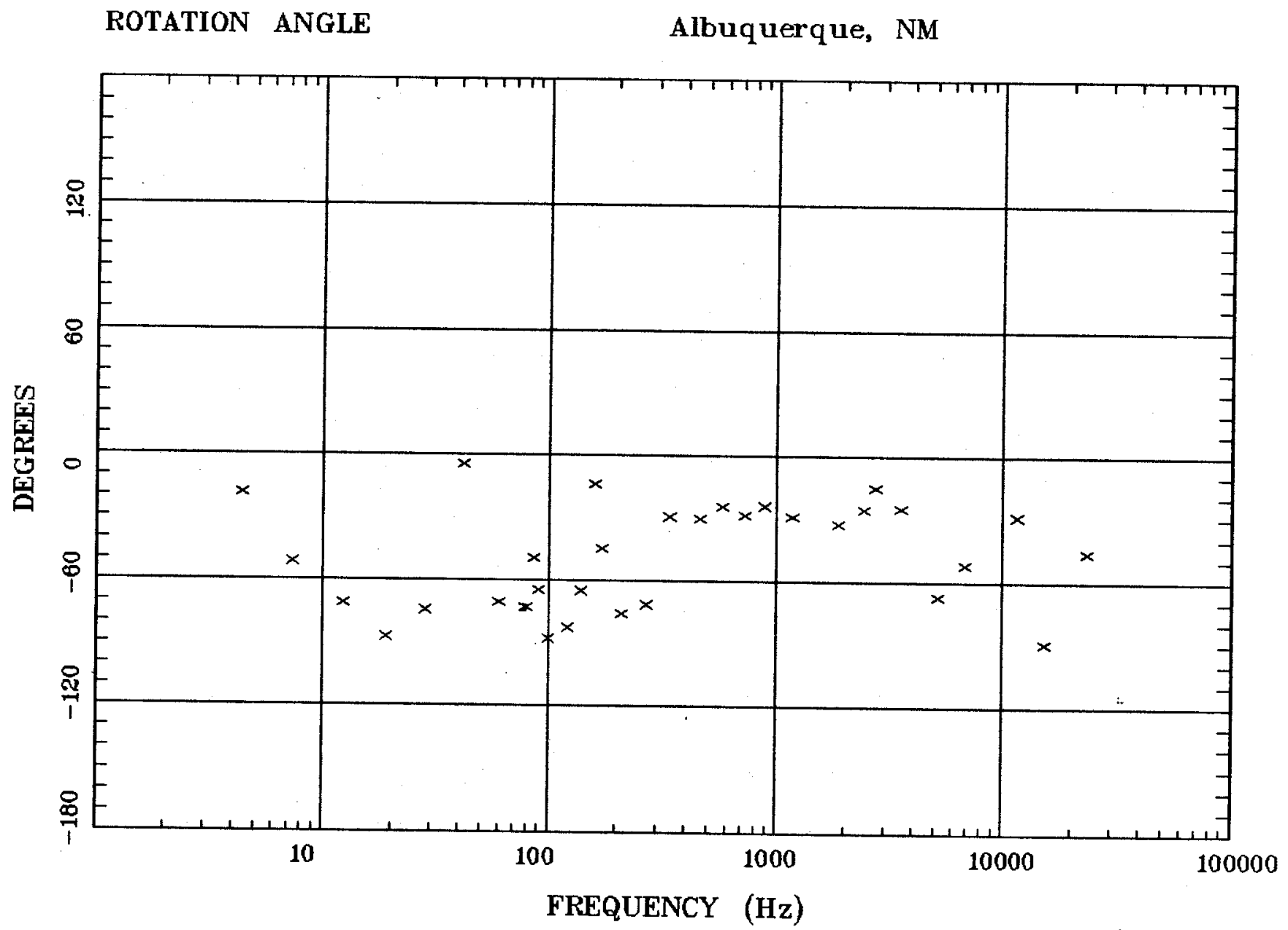

Client: City of Albuquerque Remote: $n$

Acquired: 12:5 May 07, 1998 Survey Co:USGS
Rotation:

Filename: rr11all.avg

Channels: Ch1 Ch2 Ch3 Ch4 Ch5 Ch8 Ch9

Plotted: 15:37 Mar 08, 2001

< EMI - ElectroMagnetic Instruments > 
Station 11

IMPEDANCE SKEW

Albuquerque, NM

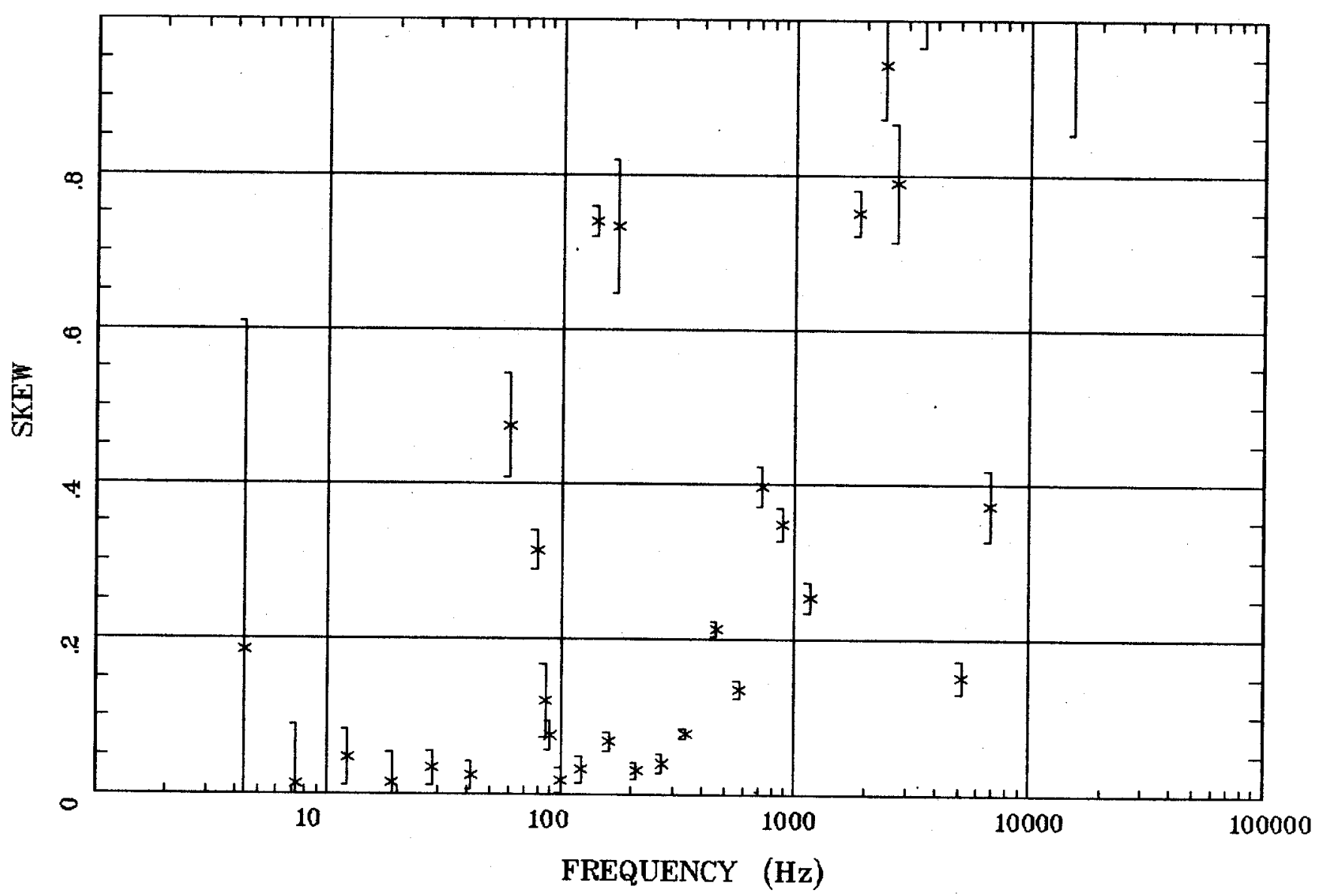

Client: City of Albuquerque Remote: $n$

Acquired: 12:5 May 07, 1998 Survey Co:USGS
Rotation:

Filename: rr11all.avg

Channels: Ch1 Ch2 Ch3 Ch4 Ch5 Ch8 Ch9

Plotted: 15:37 Mar 08, 2001

< EMI - ElectroMagnetic Instruments > 
Station 11
E MULT Coh.
Albuquerque, NM

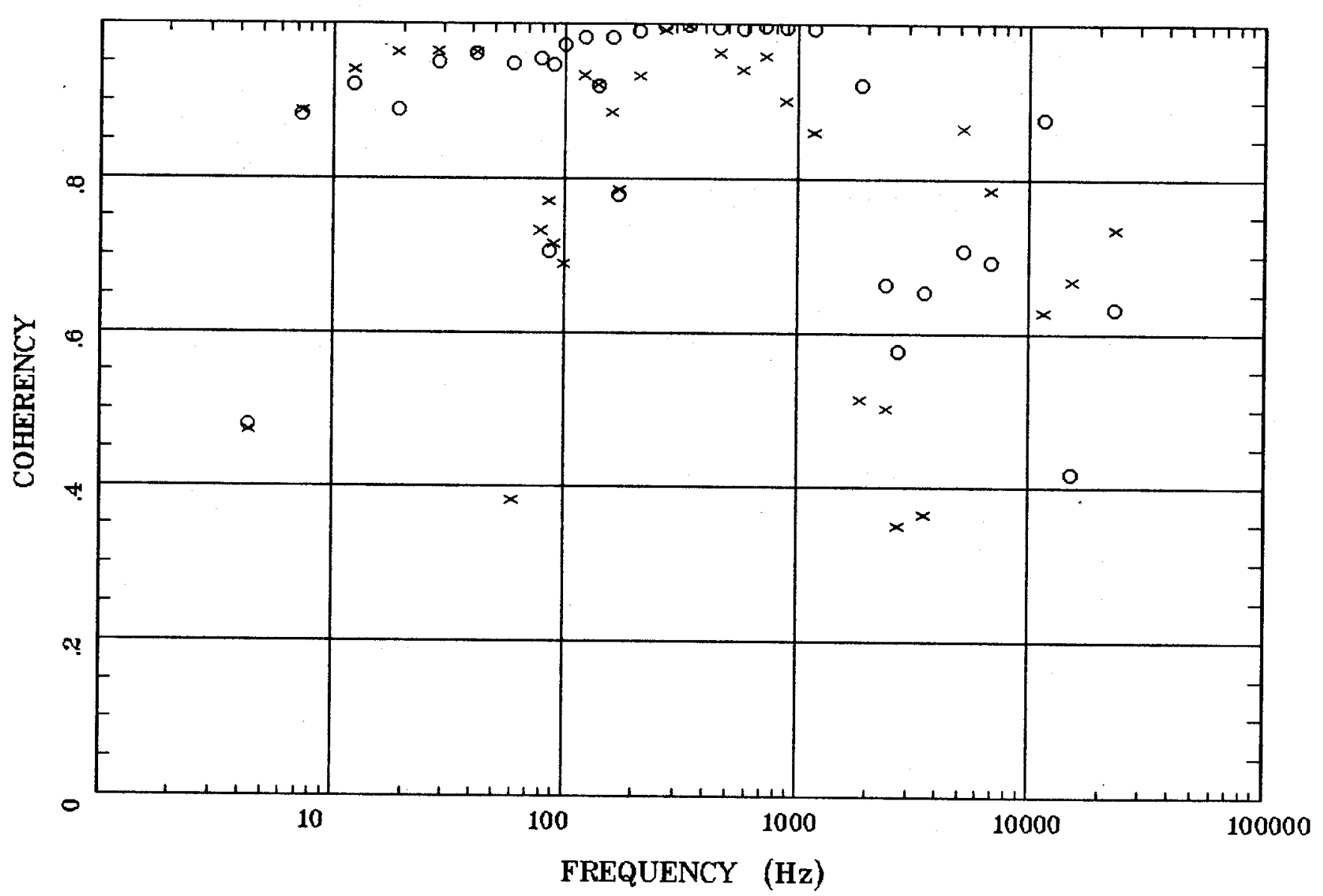

Client: City of Albuquerque

Rotation:

Remote: $\mathrm{n}$

Filename: rr11all.avg

Channels: Ch1 Ch2 Ch3 Ch4 Ch5 Ch8 Ch9

Acquired: 12:5 May 07, 1998

Plotted: 15:37 Mar 08, 2001

Survey Co:USGS

< EMI - ElectroMagnetic Instruments > 


\section{Station 11}

\section{POLAR PLOTS}

Albuquerque, NM

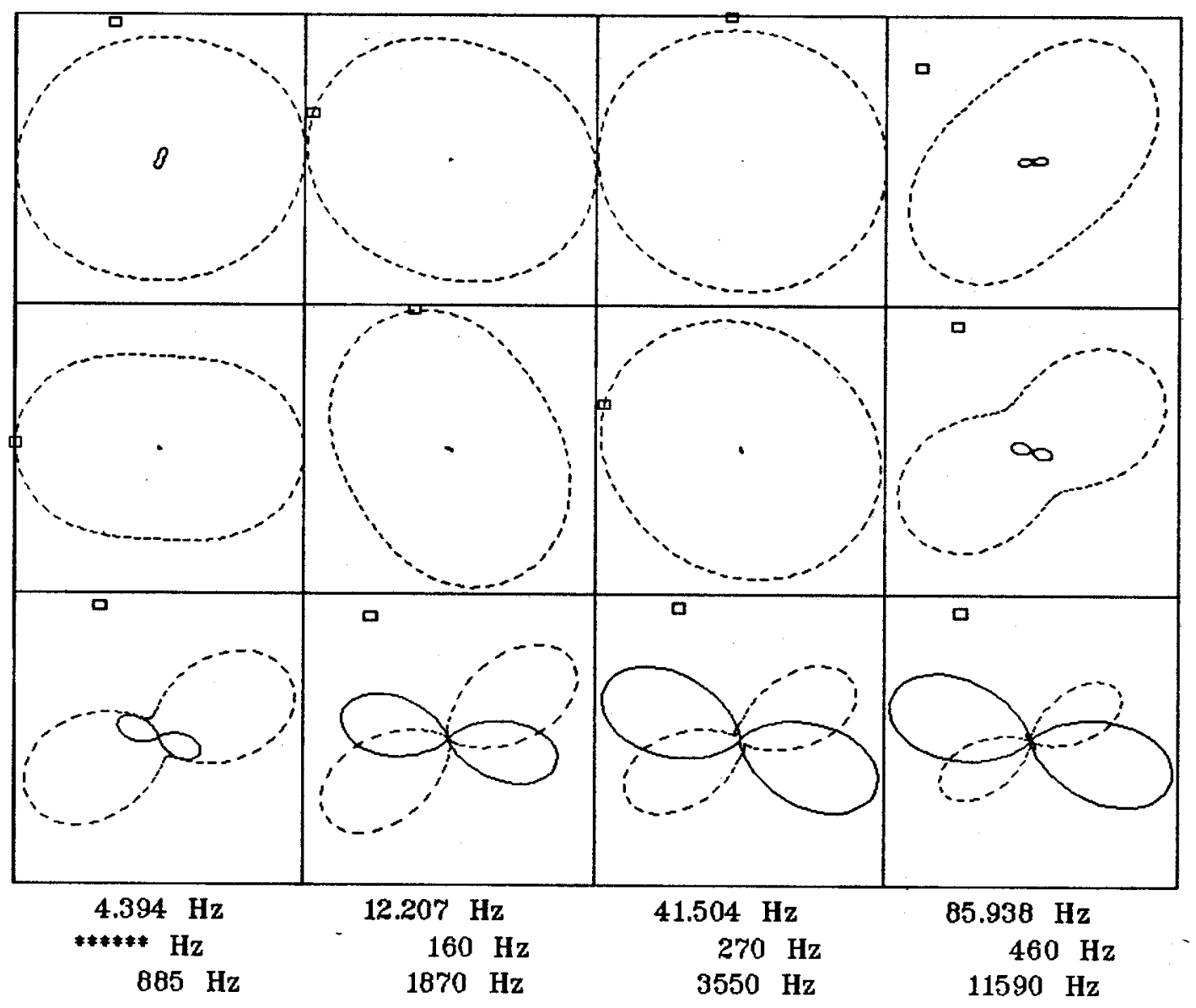

Client: City of Albuquerque Remote: $\mathbf{n}$

Acquired: 12:5 May 07, 1998

Rotation

Filename: rr11all.avg

Channels: Ch1 Ch2 Ch3 Ch4 Ch5 Ch8 Ch9

Plotted: 15:37 Mar 08, 2001

Survey Co:USGS

< EMI - ElectroMagnetic Instruments 


\section{Station 11}

TIPPER MAGNITUDE

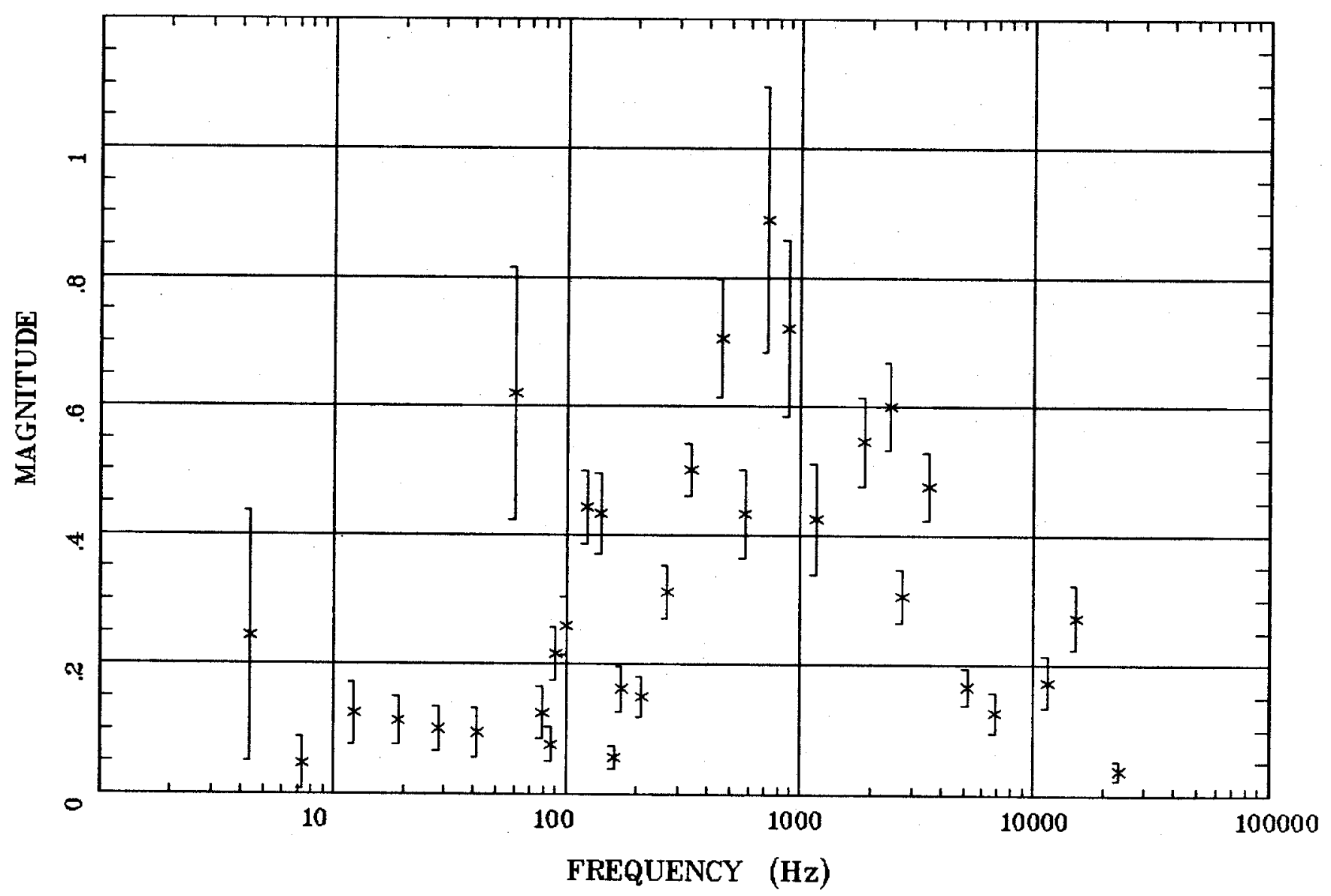

Client: City of Albuquerque Remote: $\mathbf{n}$

Acquired: 12:5 May 07, 1998 Survey Co:USGS
Rotation:

Filename: rr11all.avg

Channels: Ch1 Ch2 Ch3 Ch4 Ch5 Ch8 Ch9

Plotted: 15:37 Mar 08, 2001

$<$ EMI - ElectroMagnetic Instruments 


\section{Station 11}

TIPPER STRIKE

Albuquerque, NM

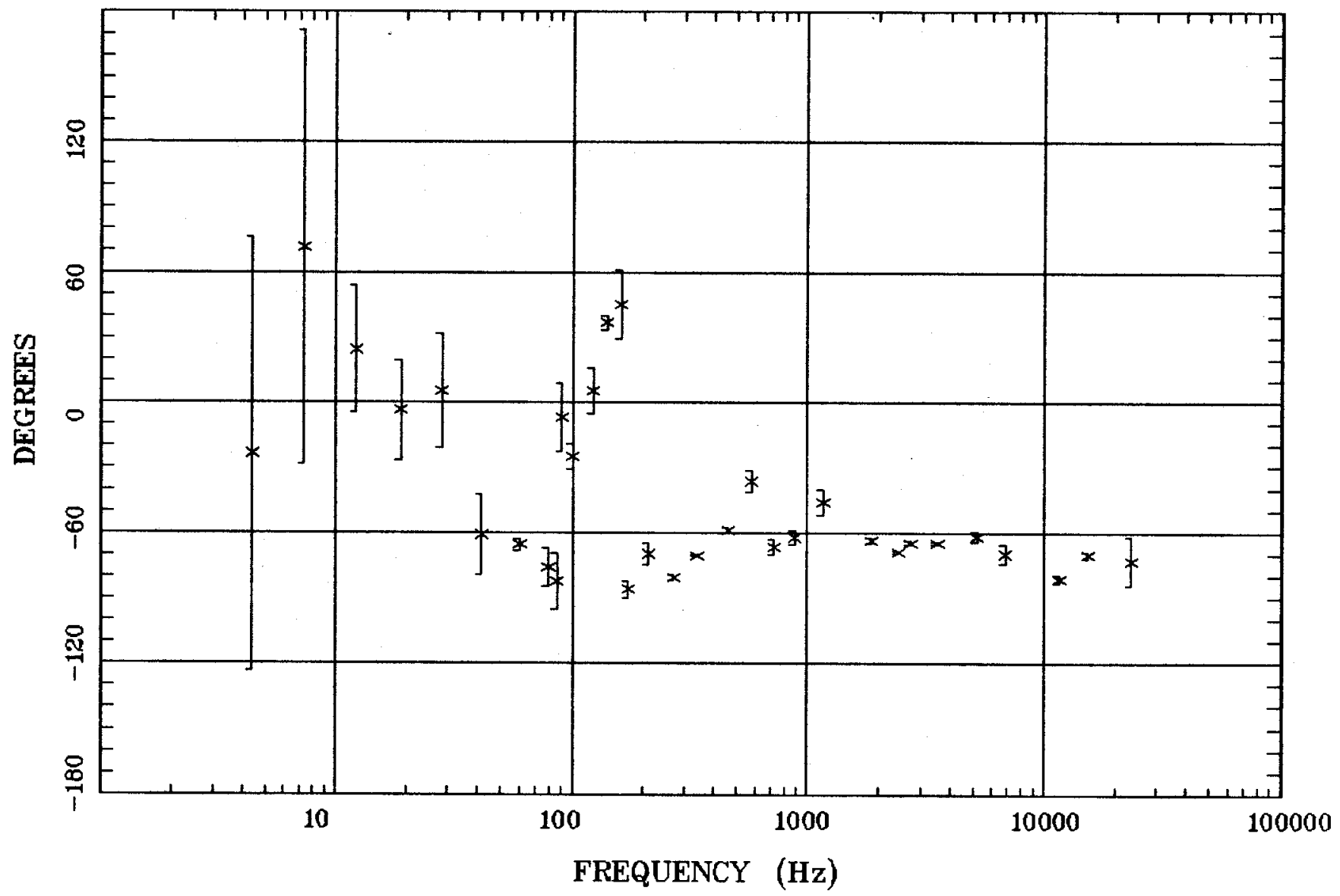

Client: City of Albuquerque Remote: $\mathbf{n}$

Acquired: 12:5 May 07, 1998 Survey Co:USGS
Rotation:

Filename: rr11all.avg

Channels: Ch1 Ch2 Ch3 Ch4 Ch5 Ch8 Ch9

Plotted: 15:37 Mar 08, 2001

< EMI - ElectroMagnetic Instruments 
Station 11

HzHx.x Coh HzHy.o

Albuquerque, NM

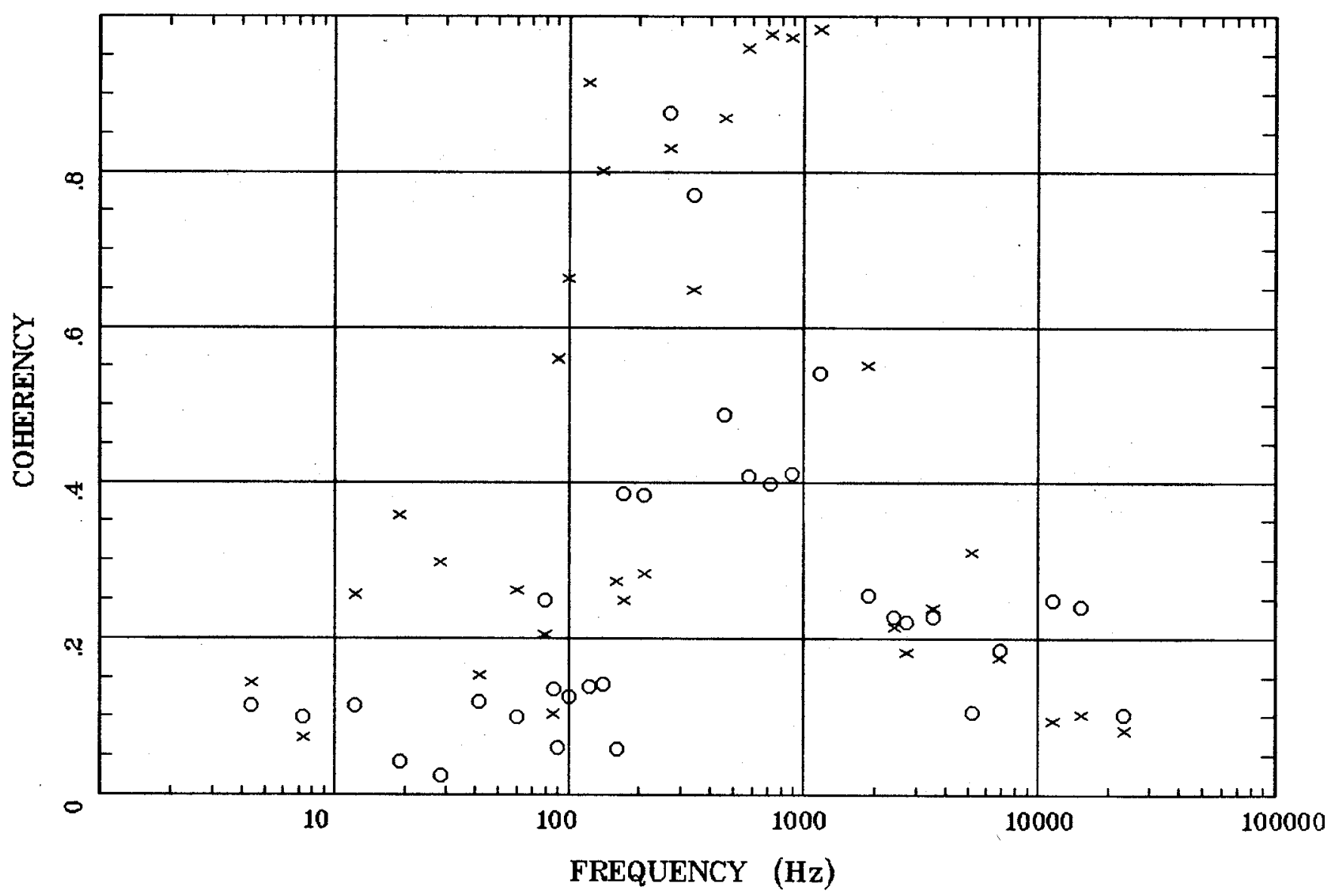

Client: City of Albuquerque Remote: $n$

Acquired: 12:5 May 07, 1998

Survey Co:USGS
Rotation:

Filename: rr11all.avg

Channels: Ch1 Ch2 Ch3 Ch4 Ch5 Ch8 Ch9

Plotted: 15:37 Mar 08, 2001

$<$ EMI - ElectroMagnetic Instruments $>$ 


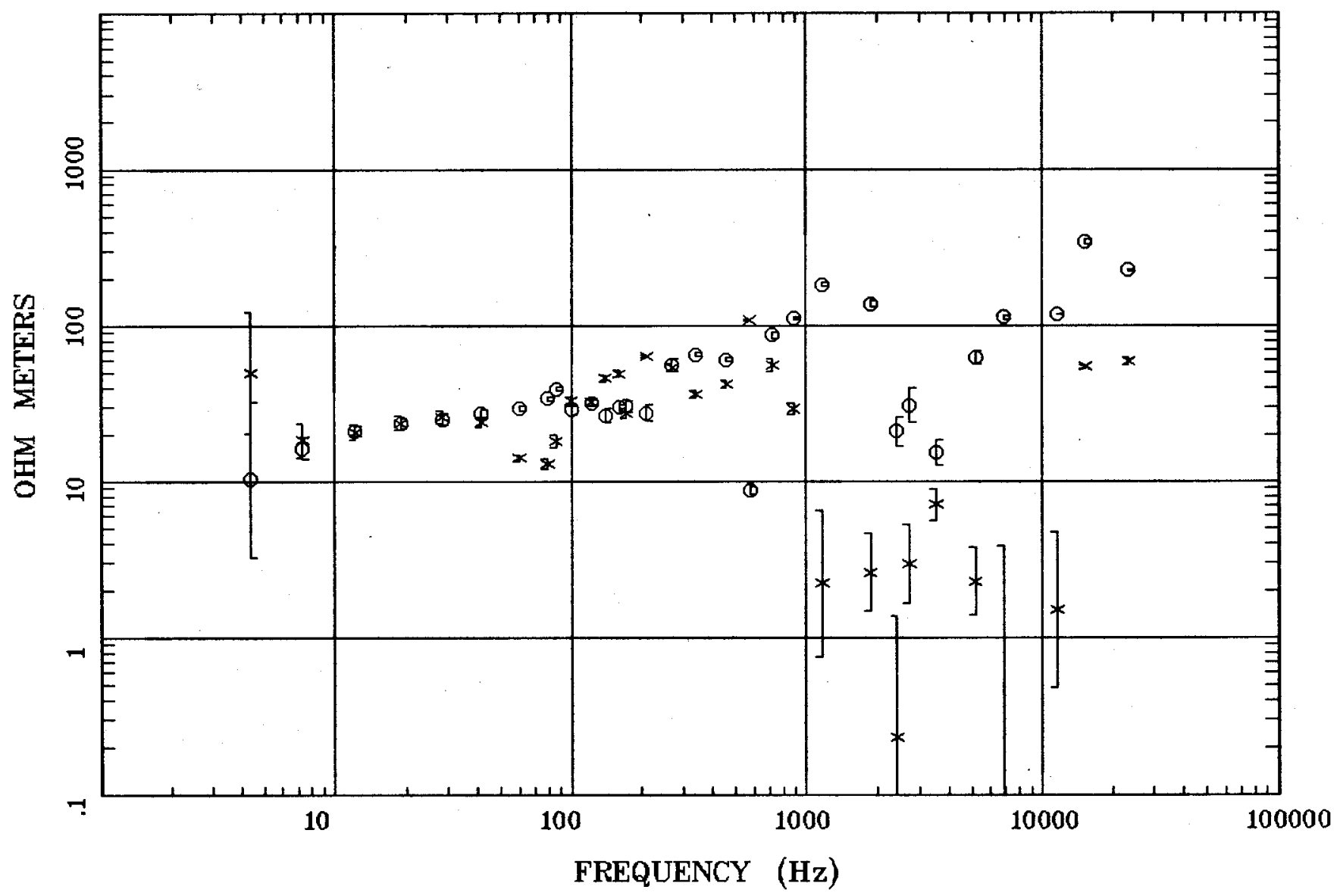

Client: City of Albuquerque Remote: $\mathrm{n}$ Acquired: 08:0 May 09, 1998 Survey Co:USGS
Rotation:

Filename: rr13a2.avg

Channels: Ch1 Ch2 Ch3 Ch4 Ch5 Ch8 Ch9 Plotted: 15:38 Mar 08, 2001

< EMI - ElectroMagnetic Instruments 
Station 13

IMPEDANCE PHASE

Albuquerque, NM

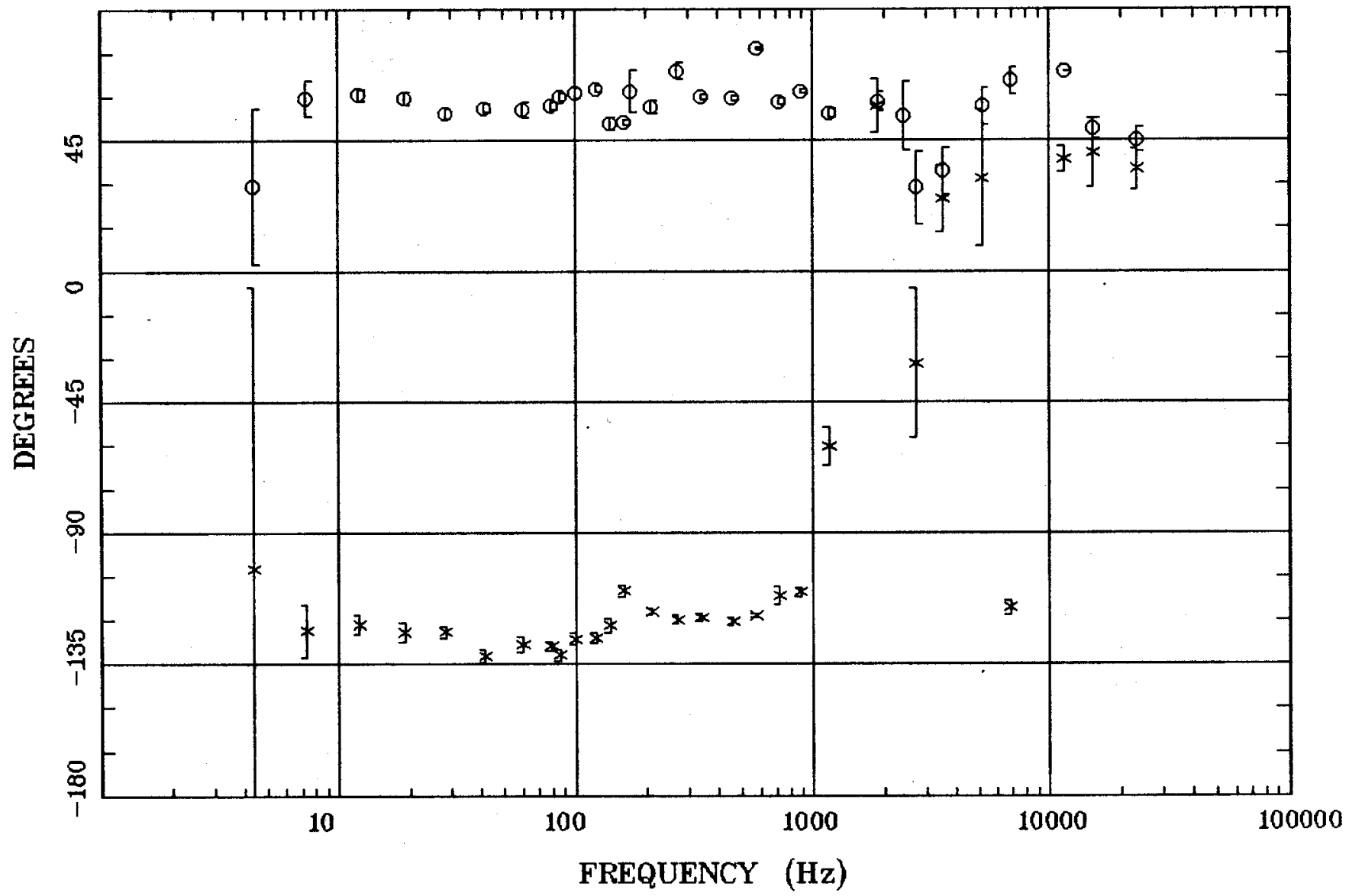

Client: City of Albuquerque Remote: $\mathbf{n}$

Acquired: 08:0 May 09, 1998 Survey Co:USGS
Rotation:

Filename: rr13a2.avg

Channels: Ch1 Ch2 Ch3 Ch4 Ch5 Ch8 Ch9

Plotted: 15:38 Mar 08, 2001

< EMI - ElectroMagnetic Instruments 


\section{Station 13}

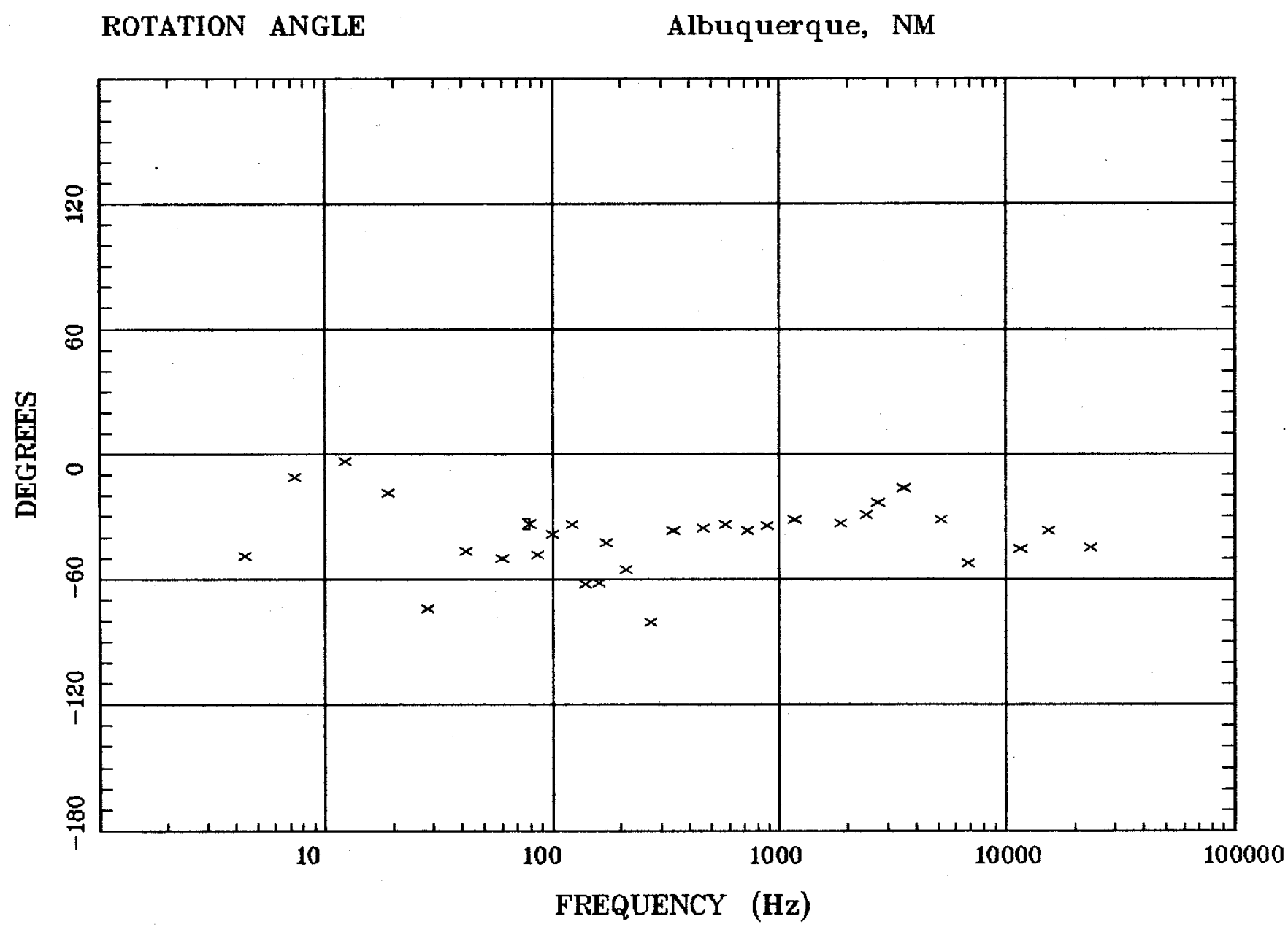

Client: City of Albuquerque Remote: $\mathbf{n}$

Rotation:

Filename: rr13a2.avg

Channels: Ch1 Ch2 Ch3 Ch4 Ch5 Ch8 Ch9

Acquired: 08:0 May 09, 1998

Plotted: 15:38 Mar 08, 2001

Survey Co:USGS

< EMI - ElectroMagnetic Instruments > 


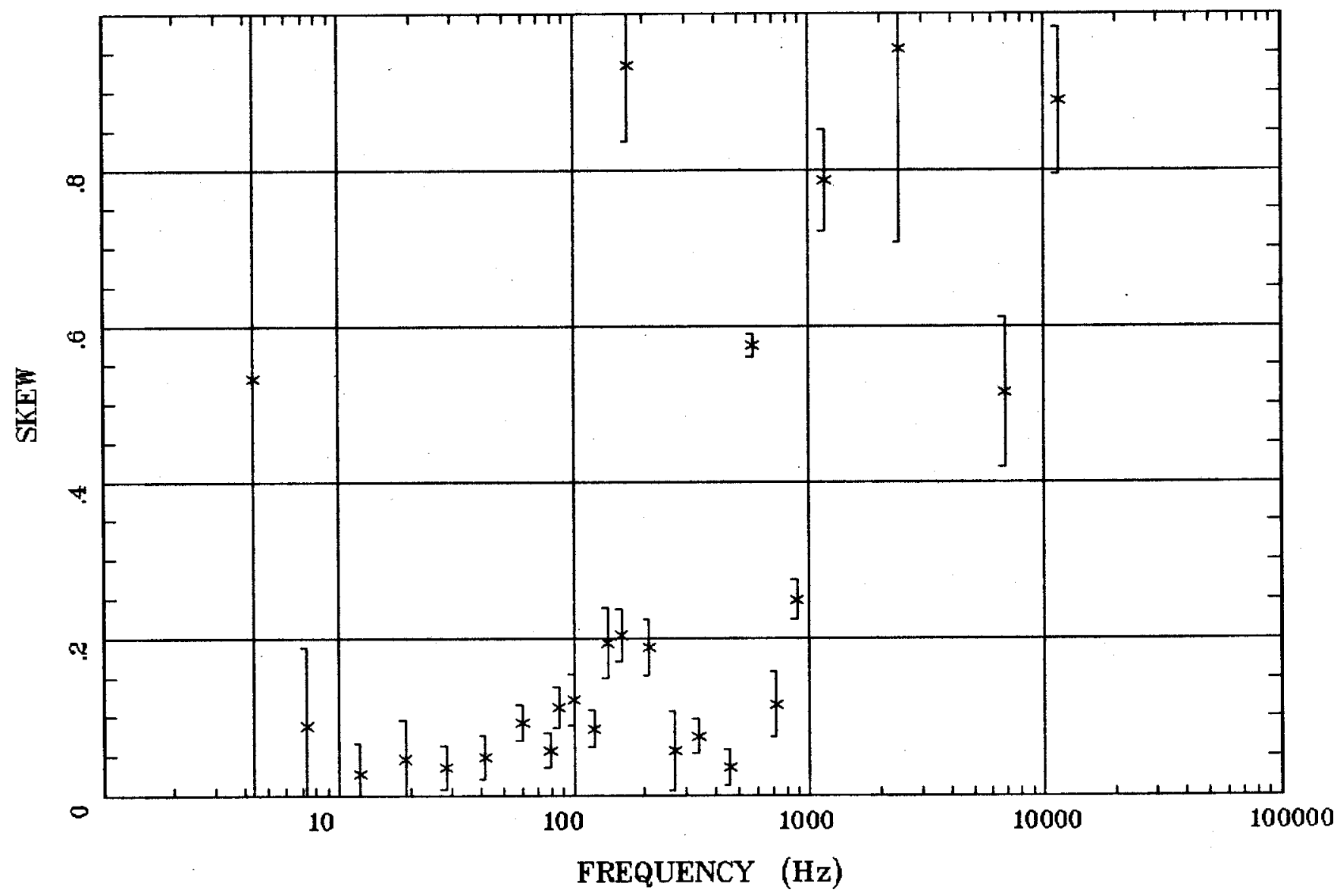

Client: City of Albuquerque Remote: $\mathbf{n}$ Acquired: 08:0 May 09, 1998 Survey Co:USGS
Rotation:

Filename: rr13a2.avg

Channels: Ch1 Ch2 Ch3 Ch4 Ch5 Ch8 Ch9 Plotted: 15:38 Mar 08, 2001

< EMI - ElectroMagnetic Instruments > 
Station 13

E MULT Coh.

Albuquerque, NM

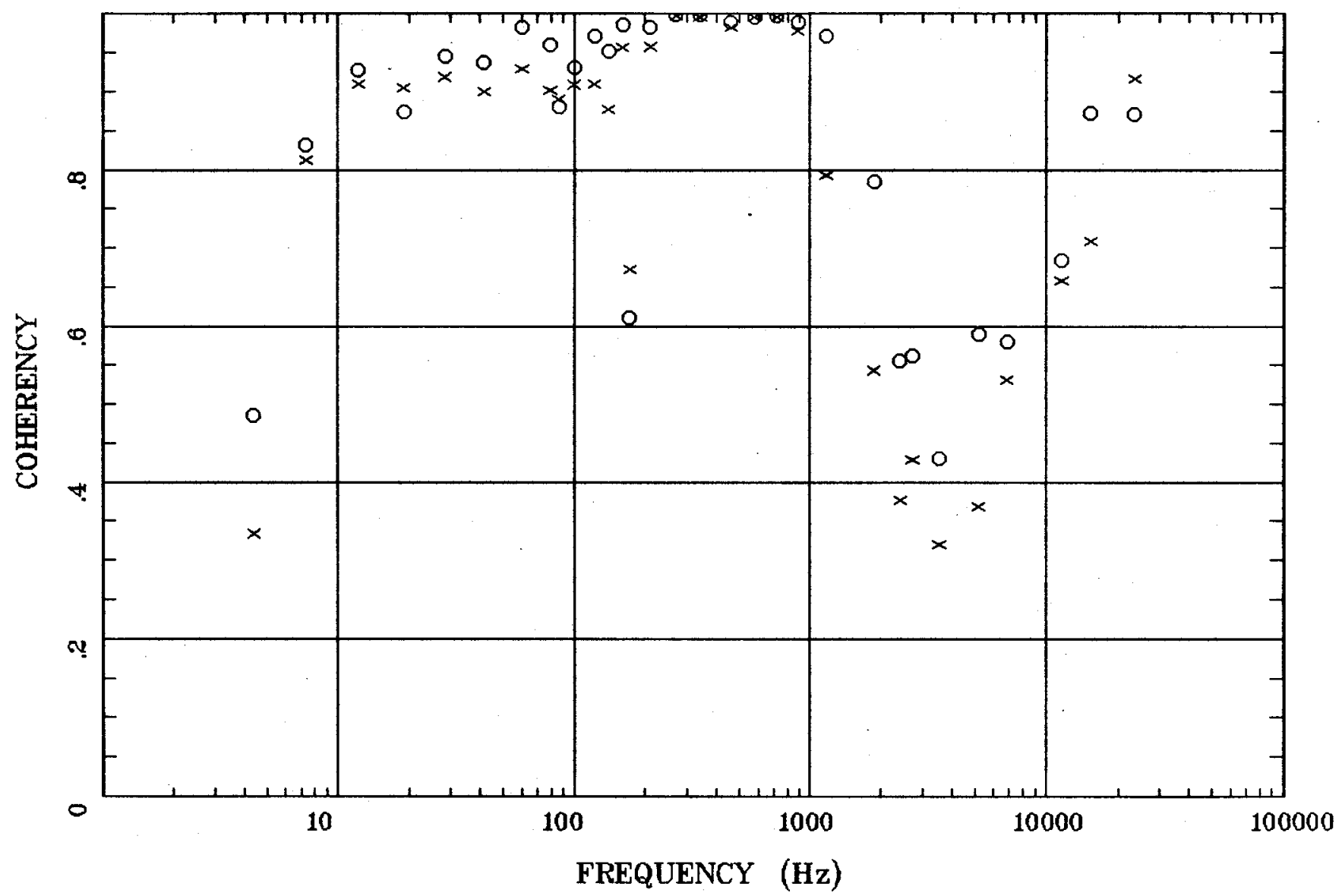

Client: City of Albuquerque Remote: $\mathrm{n}$

Acquired: 08:0 May 09, 1998 Survey Co:USGS
Rotation:

Filename: rr13a2.avg

Channels: Ch1 Ch2 Ch3 Ch4 Ch5 Ch8 Ch9 Plotted: 15:36 Mar 08, 2001

< EMI - ElectroMagnetic Instruments 
Station 13

POLAR PLOTS

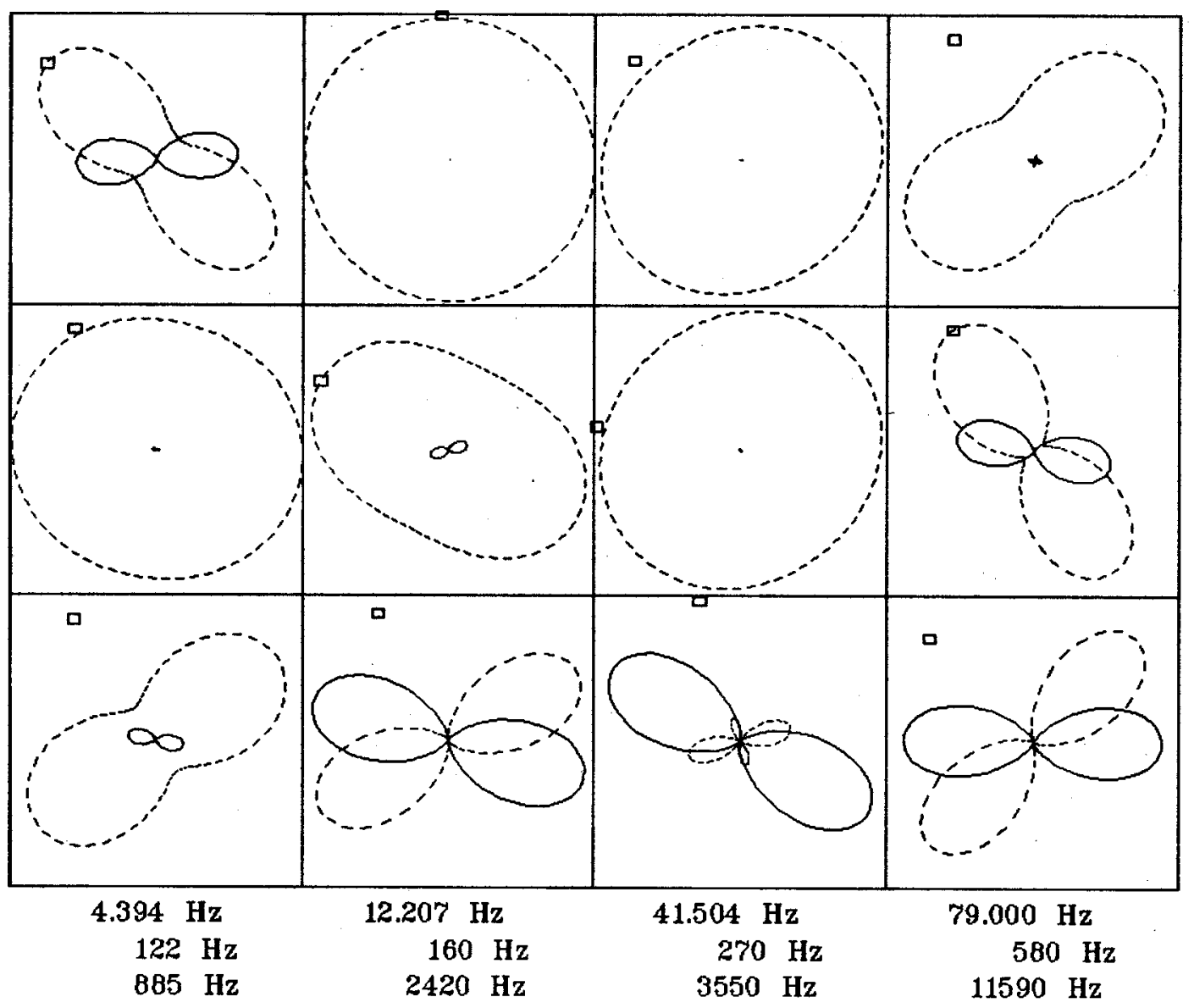

Client: City of Albuquerque

Remote: $\mathbf{n}$

Acquired: 08:0 May 09, 1998 Survey Co:USGS
Albuquerque, NM

Rotation:

Filename: rr13a2.avg

Channels: Ch1 Ch2 Ch3 Ch4 Ch5 Ch8 Ch9

Plotted: 15:38 Mar 08, 2001

< EMI - ElectroMagnetic Instruments 


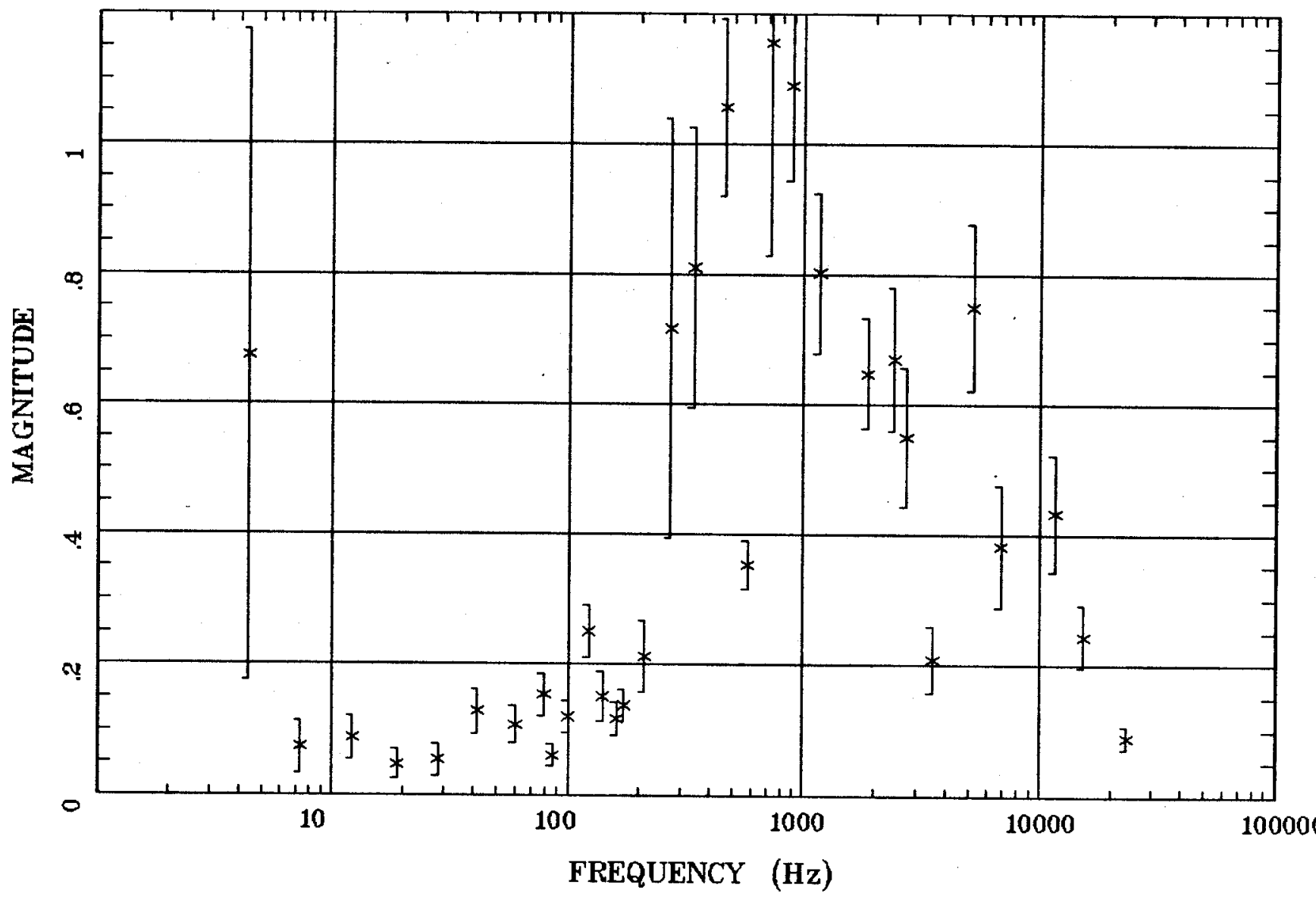

Client: City of Albuquerque Remote: $\mathbf{n}$

Acquired: 08:0 May 09, 1998 Survey Co:USGS
Rotation:

Filename: rr13a2.avg

Channels: Ch1 Ch2 Ch3 Ch4 Ch5 Ch8 Ch9

Plotted: 15:38 Mar 08, 2001

< EMI - ElectroMagnetic Instruments > 


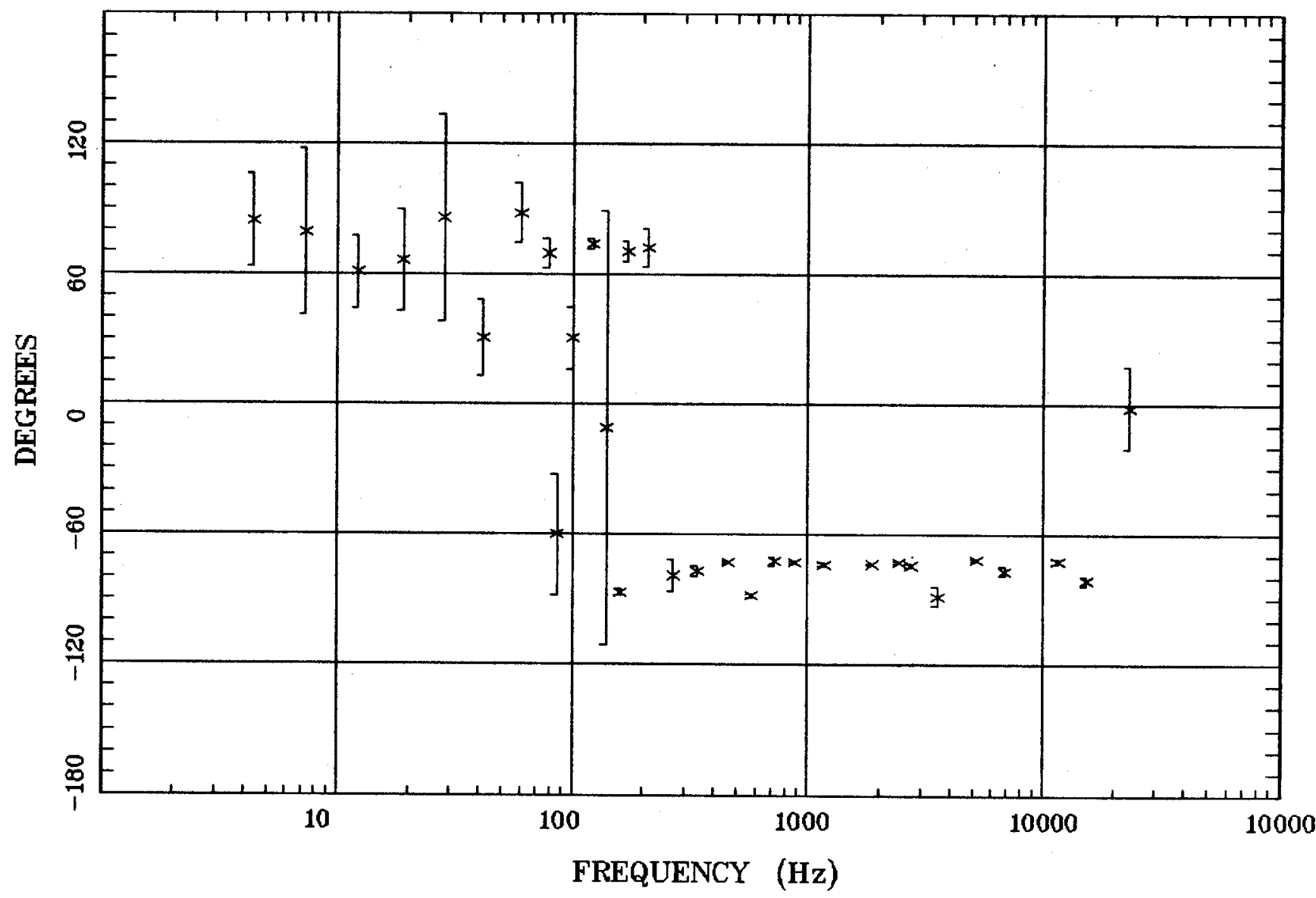

Client: City of Albuquerque Remote: $n$

Acquired: 08:0 May 09, 1998 Survey Co:USGS
Rotation:

Filename: $\operatorname{rr} 13 \mathrm{a} 2 . \mathrm{avg}$

Channels: Ch1 Ch2 Ch3 Ch4 Ch5 Ch8 Ch9 Plotted: 15:38 Mar 08, 2001

< EMI - ElectroMagnetic Instruments 
HzHx.x Coh HzHy.o

Albuquerque, NM

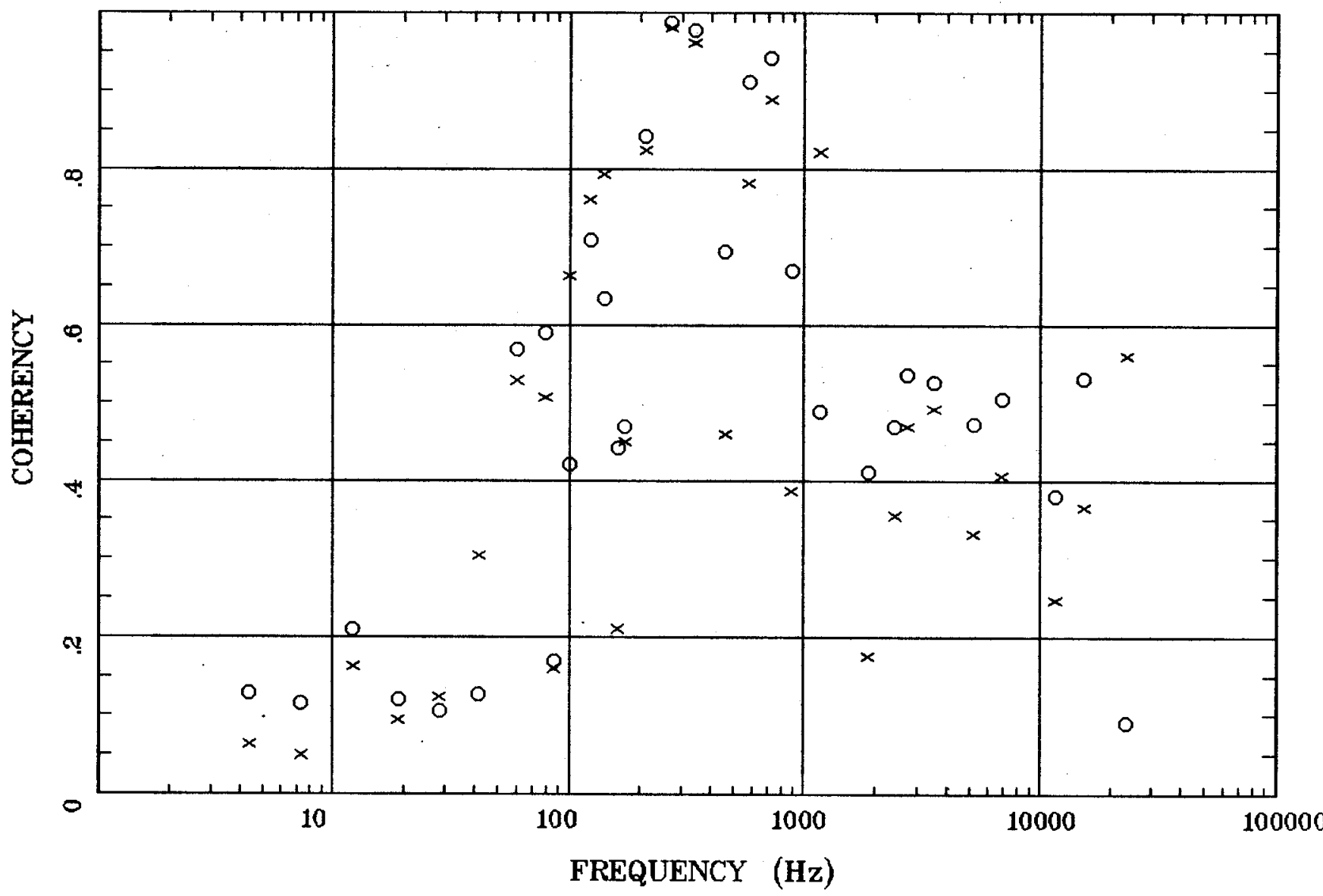

Client: City of Albuquerque Remote: $\mathrm{n}$

Acquired: 08:0 May 09, 1998 Survey Co:USGS
Rotation:

Filename: rr13a2.avg

Channels: Ch1 Ch2 Ch3 Ch4 Ch5 Ch8 Ch9

Plotted: 15:38 Mar 08, 2001

< EMI - ElectroMagnetic Instruments > 


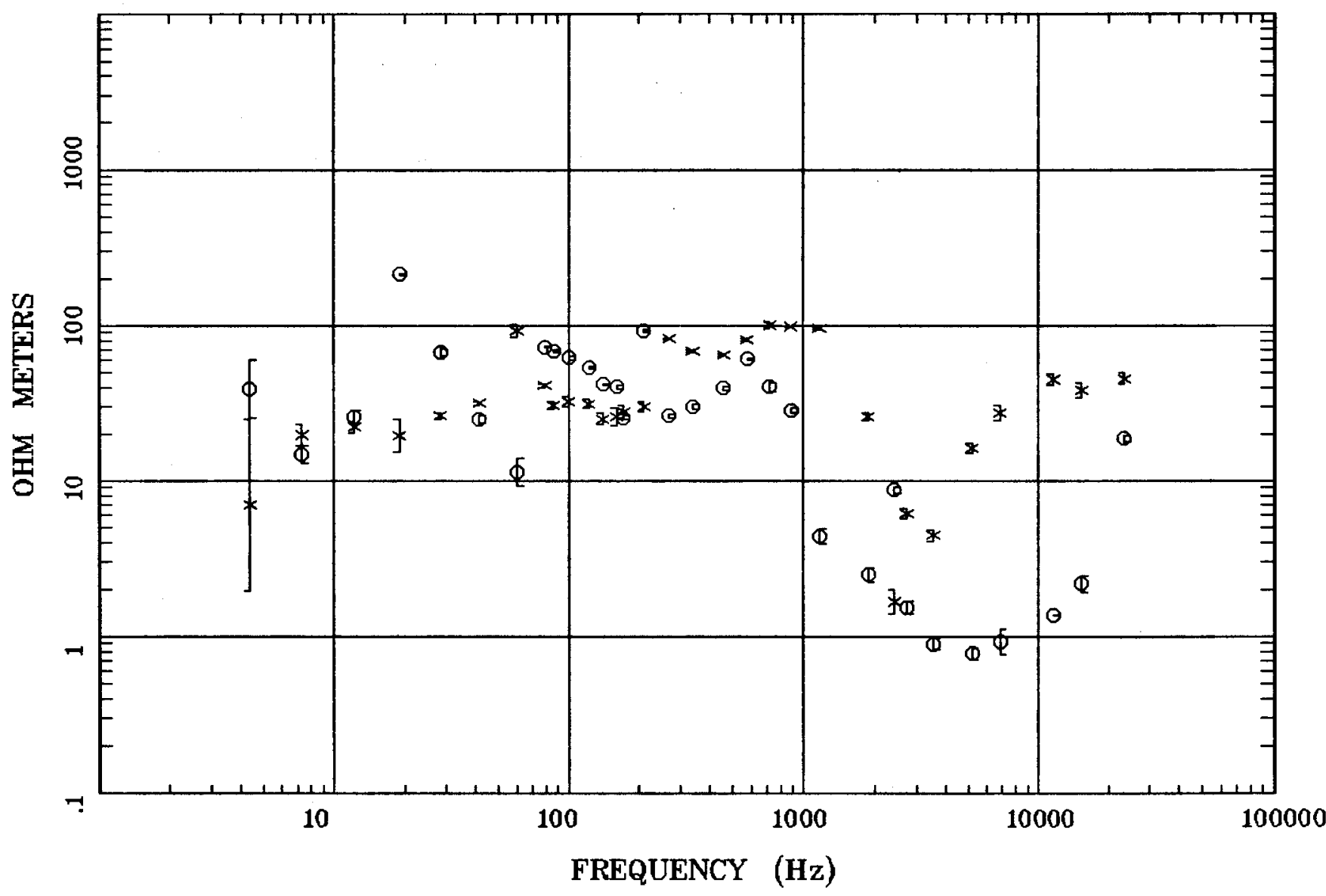

Client: City of Albuquerque Remote: $\mathbf{n}$ Acquired: 11:0 May 09, 1998 Survey Co:USGS
Rotation:

Filename: rr14a2.avg

Channels: Ch1 Ch2 Ch3 Ch4 Ch5 Ch8 Ch9 Platted: 15:38 Mar 08, 2001

< EMI - ElectroMagnetic Instruments > 


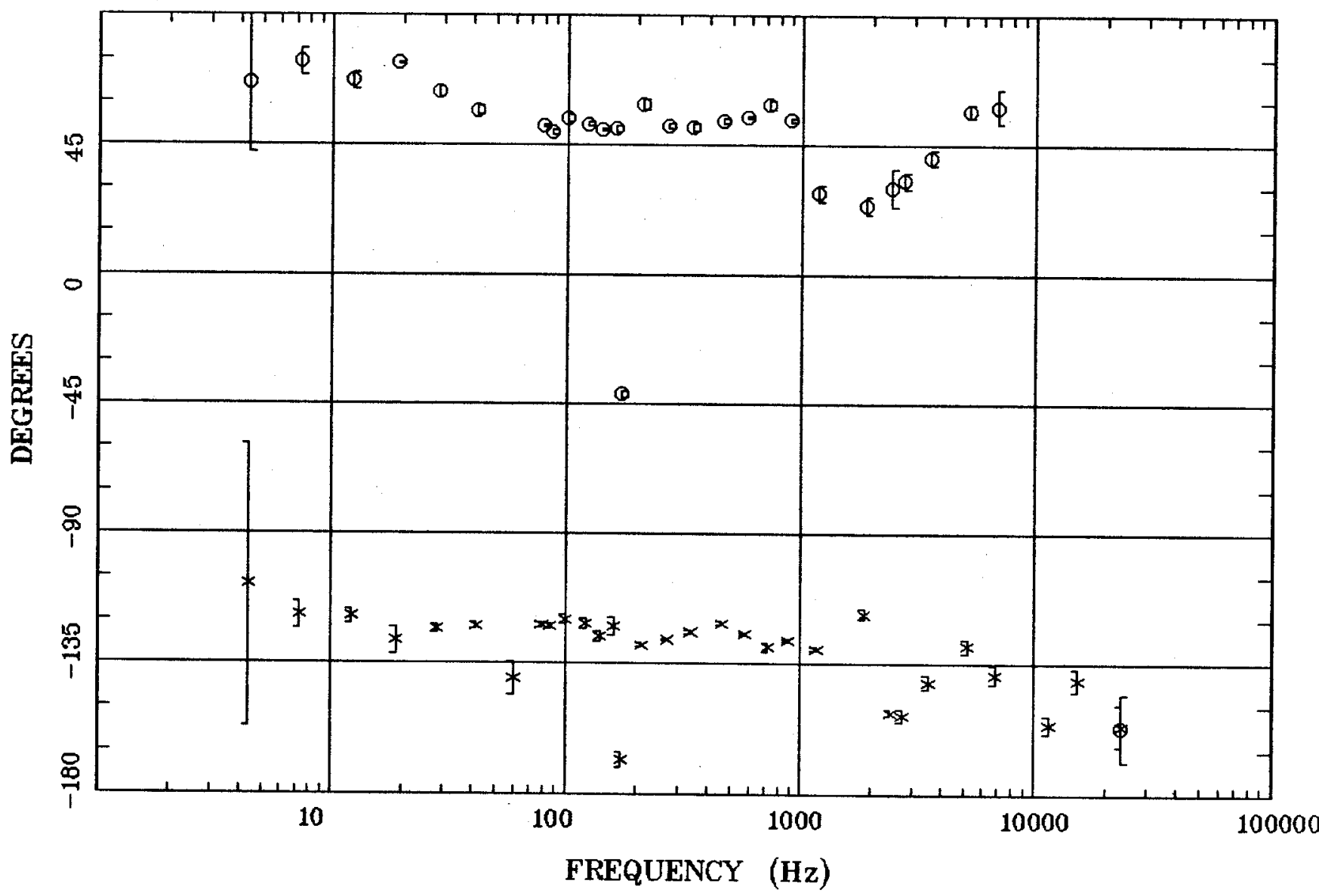

Client: City of Albuquerque Remote: $\mathbf{n}$ Acquired: 11:0 May 09, 1998 Survey Co:USGS
Rotation:

Filename: rr14a2.avg

Channels: Ch1 Ch2 Ch3 Ch4 Ch5 Ch8 Ch9 Plotted: 15:38 Mar 08, 2001

< EMI - ElectroMagnetic Instruments > 


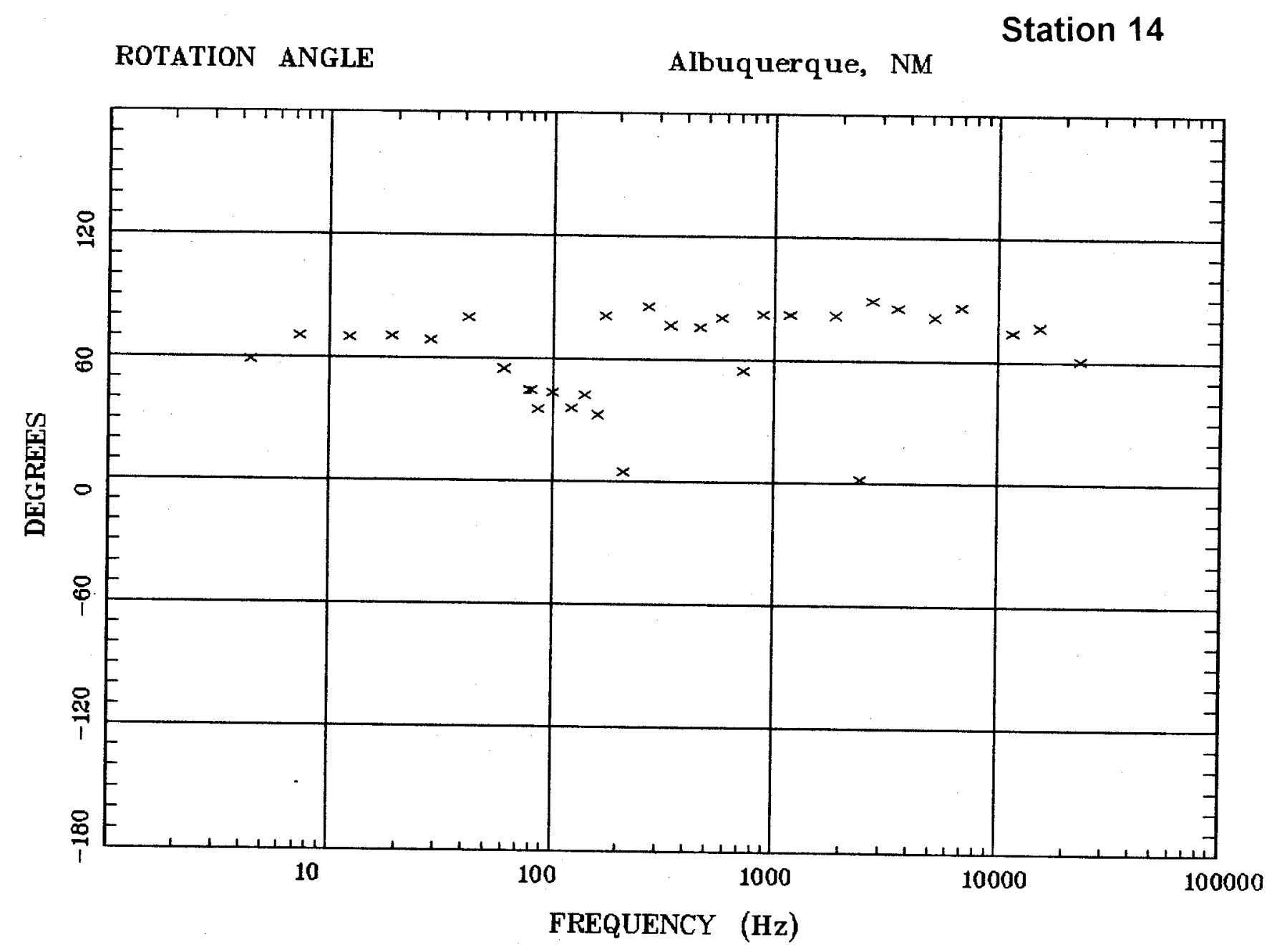

Client: City of Albuquerque

Rotation:

Remote: $\mathrm{n}$

Filename: rr14az.avg

Acquired: 11:0 May 09, 1998

Channels: Ch1 Ch2 Ch3 Ch4 Ch5 Ch8 Ch9

Survey Co:USGS

Plotted: 15:38 Mar 08, 2001

< EMI - ElectroMagnetic Instruments 


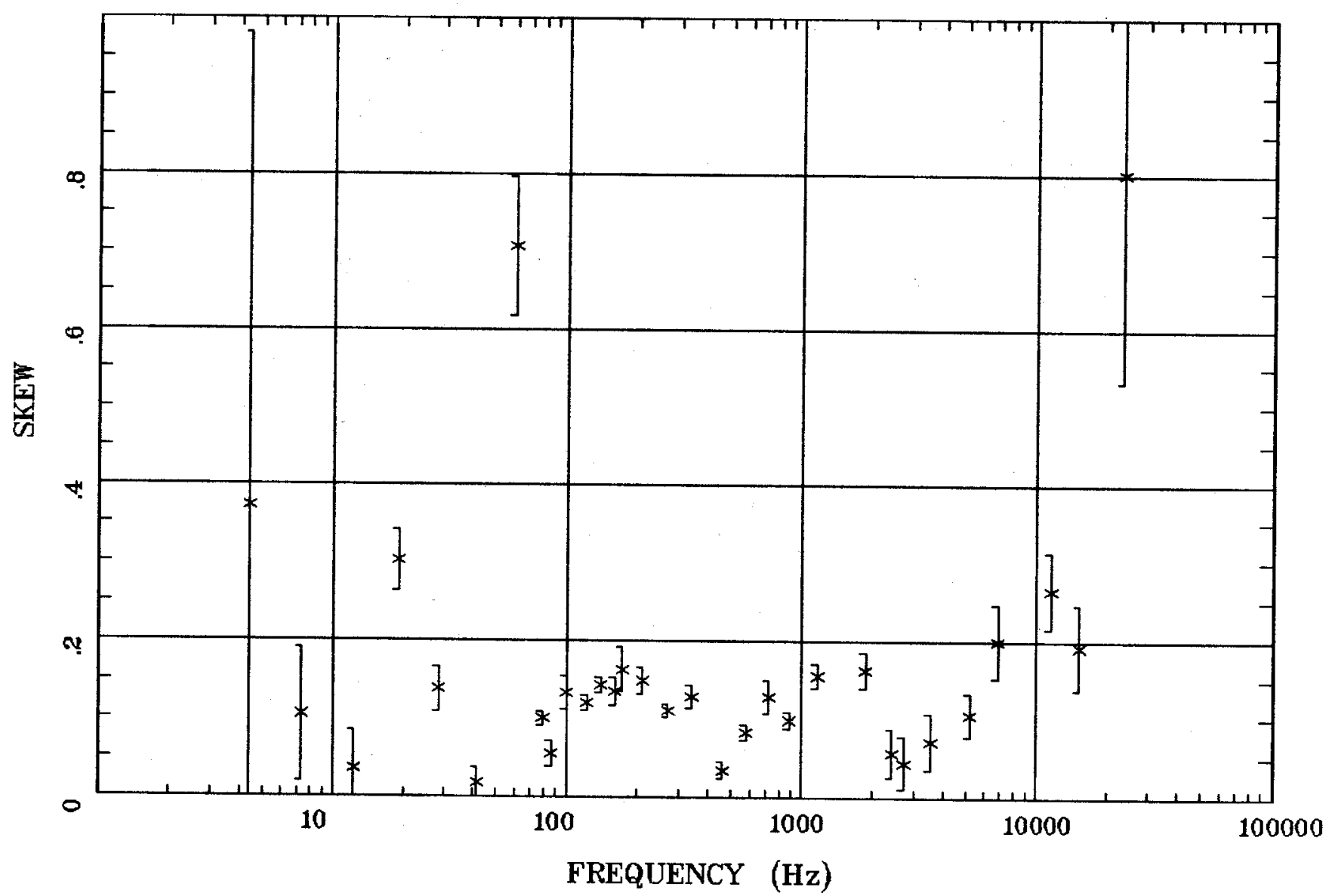

Client: City of Albuquerque Remote: $\mathbf{n}$

Acquired: 11:0 May 09, 1998 Survey Co:USGS
Rotation:

Filename: rr14a2.avg

Channels: Ch1 Ch2 Ch3 Ch4 Ch5 Ch8 Ch9

Plotted: 15:38 Mar 08, 2001

$<$ EMI - ElectroMagnetic Instruments 


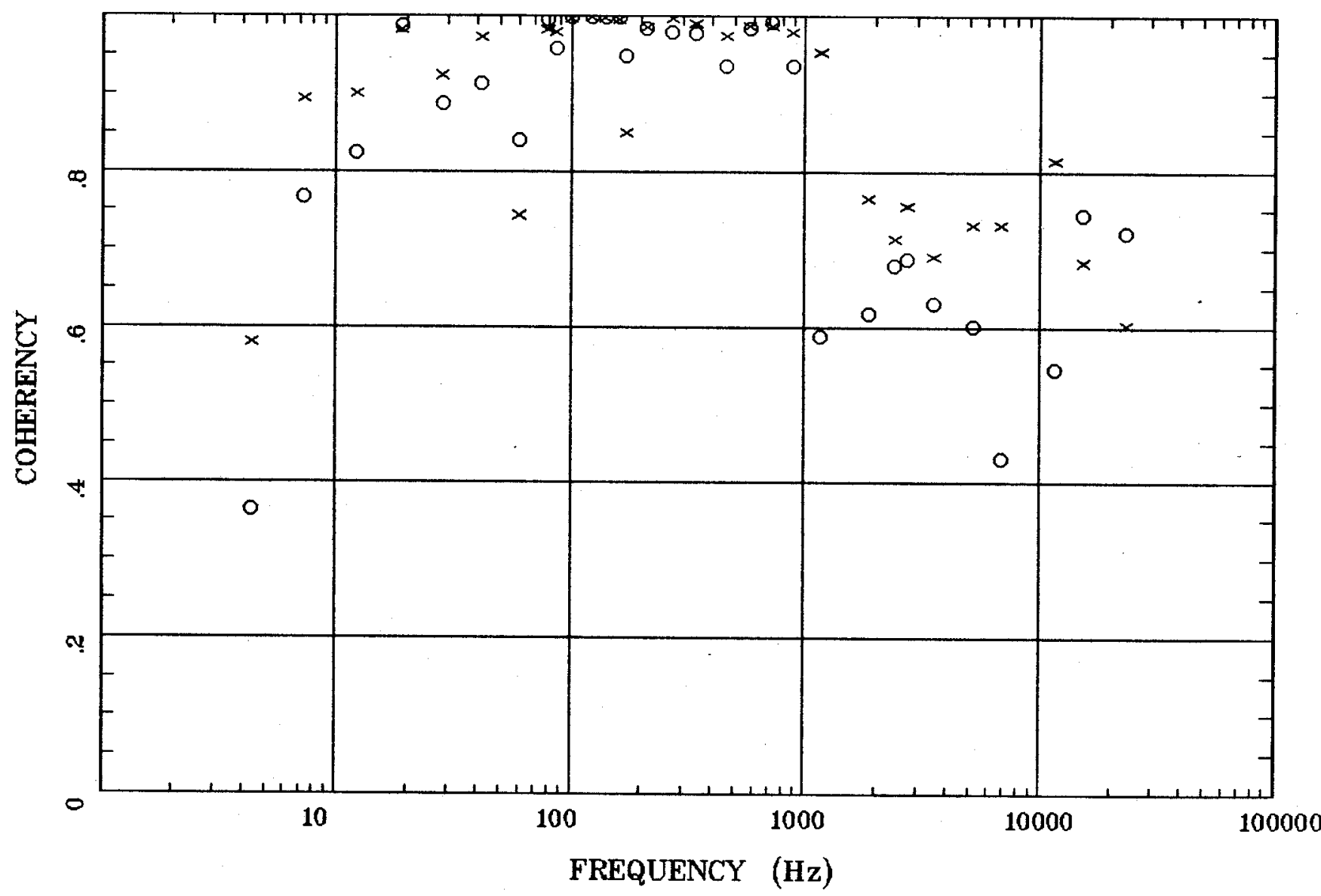

Client: City of Albuquerque Remote: $\mathrm{n}$

Acquired: 11:0 May 09, 1998 Survey Co:USGS
Rotation:

Filename: rr14a2.avg

Channels: Ch1 Ch2 Ch3 Ch4 Ch5 Ch8 Ch9

Plotted: 15:38 Mar 08, 2001

< EMI - ElectroMagnetic Instruments > 


\section{POLAR PLOTS}

Albuquerque, NM

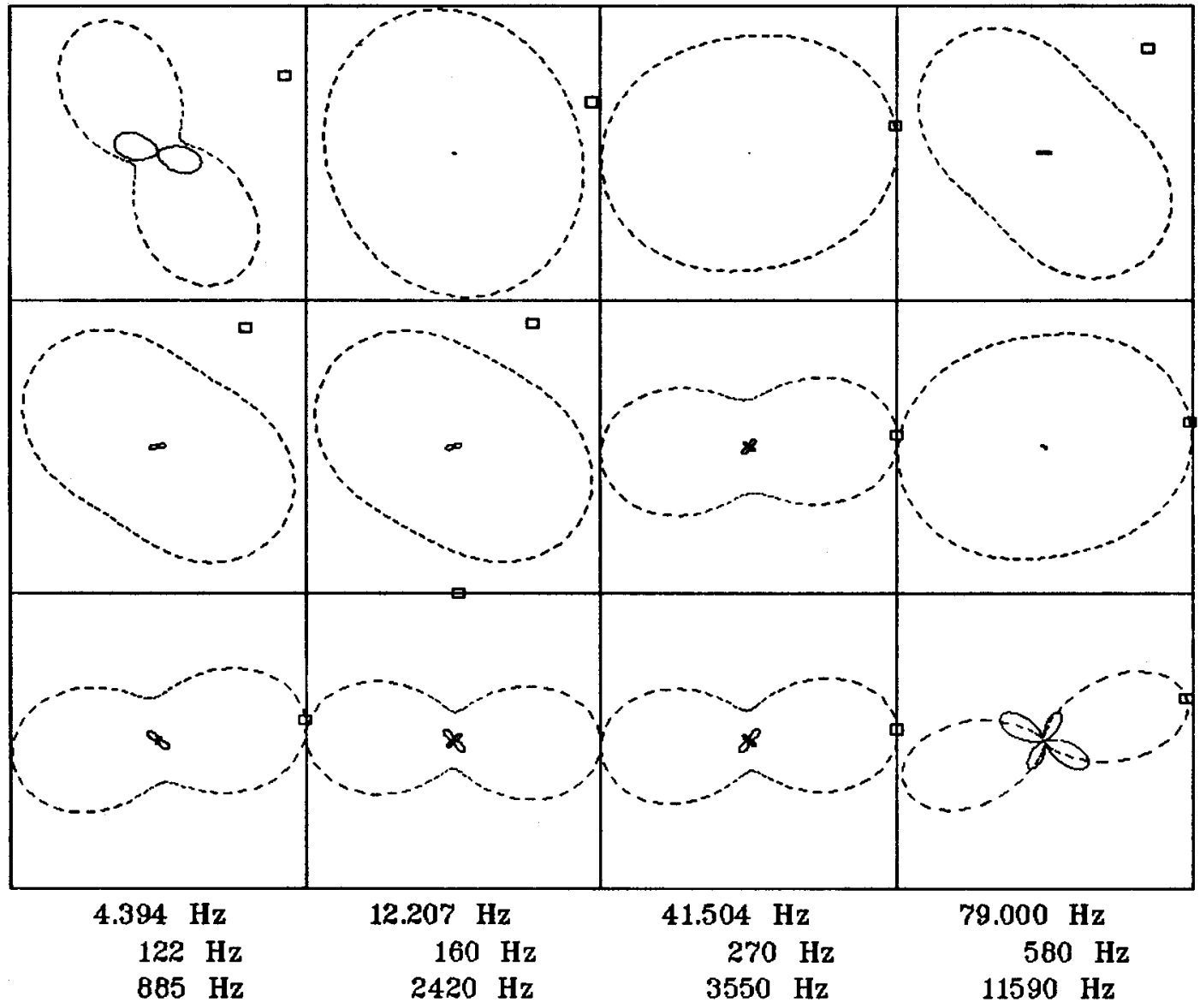

Client: City of Albuquerque Remote: $\mathbf{n}$

Acquired: 11:0 May 09, 1998 Survey Co:USGS
Rotation:

Filename: rr14a2.avg

Channels: Ch1 Ch2 Ch3 Ch4 Ch5 Ch8 Ch9 Plotted: 15:38 Mar 08, 2001

< EMI - ElectroMagnetic Instruments > 
Albuquerque, NM

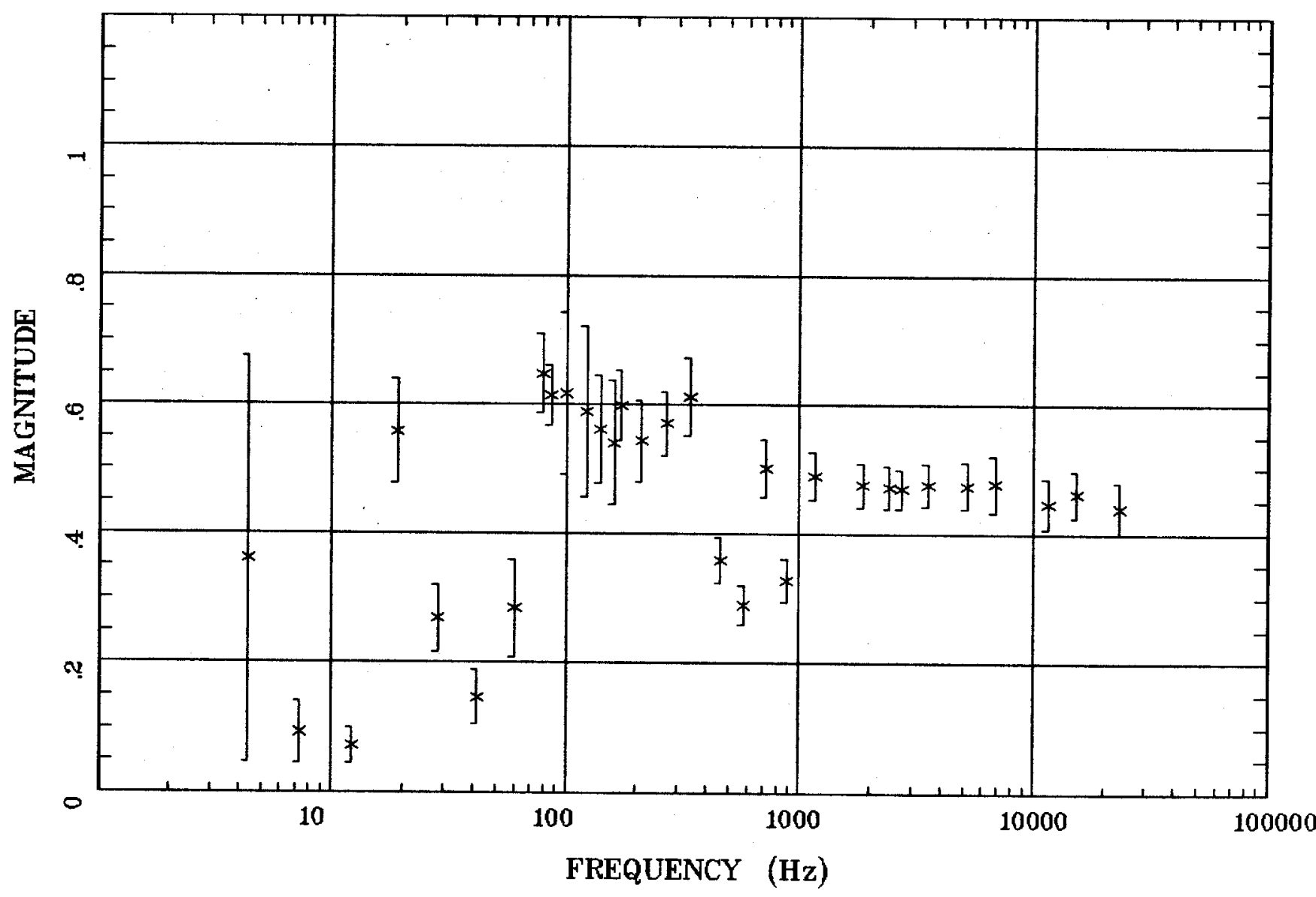

Client: City of Albuquerque Remote: $\mathbf{n}$ Acquired: 11:0 May 09, 1998 Survey Co:USGS

\section{Rotation:}

Filename: rr14a2.avg

Channels: Ch1 Ch2 Ch3 Ch4 Ch5 Ch8 Ch9 Plotted: 15:38 Mar 08, 2001

< EMI - ElectroMagnetic Instruments > 
Albuquerque, NM

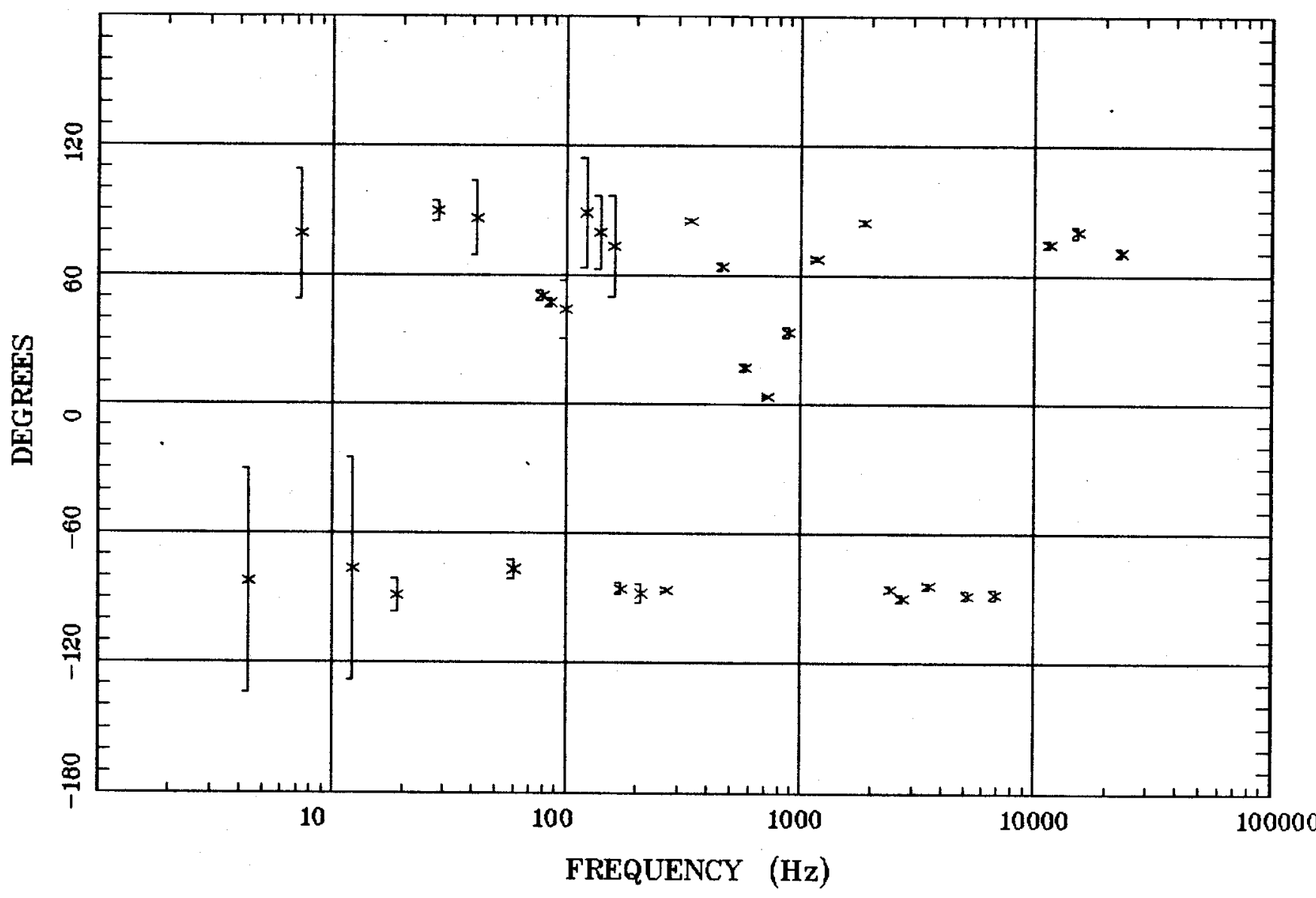

Client: City of Albuquerque Remote: $\mathbf{n}$

Acquired: 11:0 May 09, 1998 Survey Co:USGS
Rotation:

Filename: rr14a2.avg

Channels: Ch1 Ch2 Ch3 Ch4 Ch5 Ch8 Ch9 Plotted: 15:38 Mar 08, 2001

< EMI - ElectroMagnetic Instruments > 
HzHx.x Coh HzHy.o

Albuquerque, NM

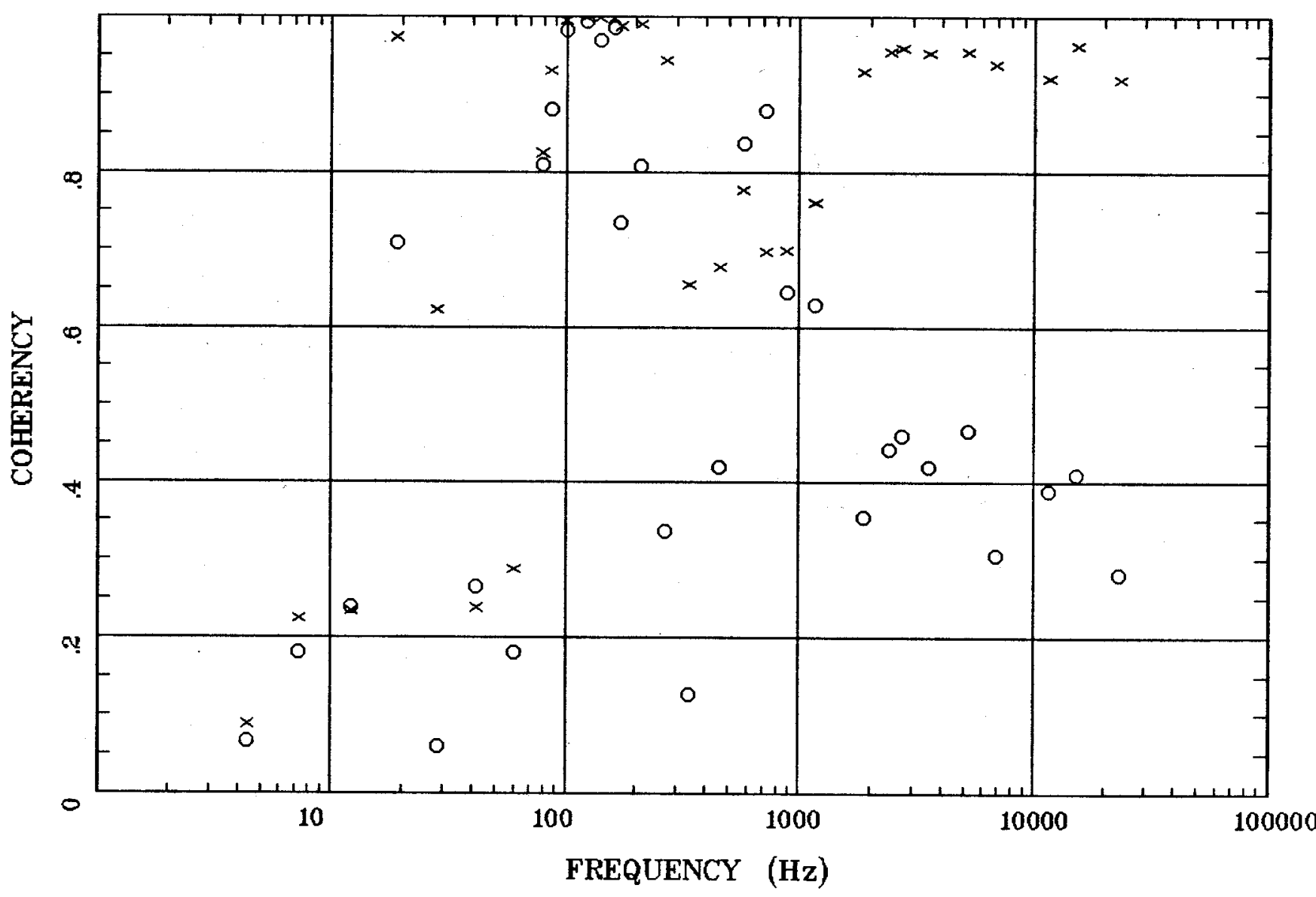

Client: City of Albuquerque Remote: $\mathbf{n}$

Acquired: 11:0 May 09, 1998 Survey Co:USGS
Rotation:

Filename: rr14az.avg

Channels: Ch1 Ch2 Ch3 Ch4 Ch5 Ch8 Ch9 Platted: 15:38 Mar 08, 2001

$<$ EMI - ElectroMagnetic Instruments > 


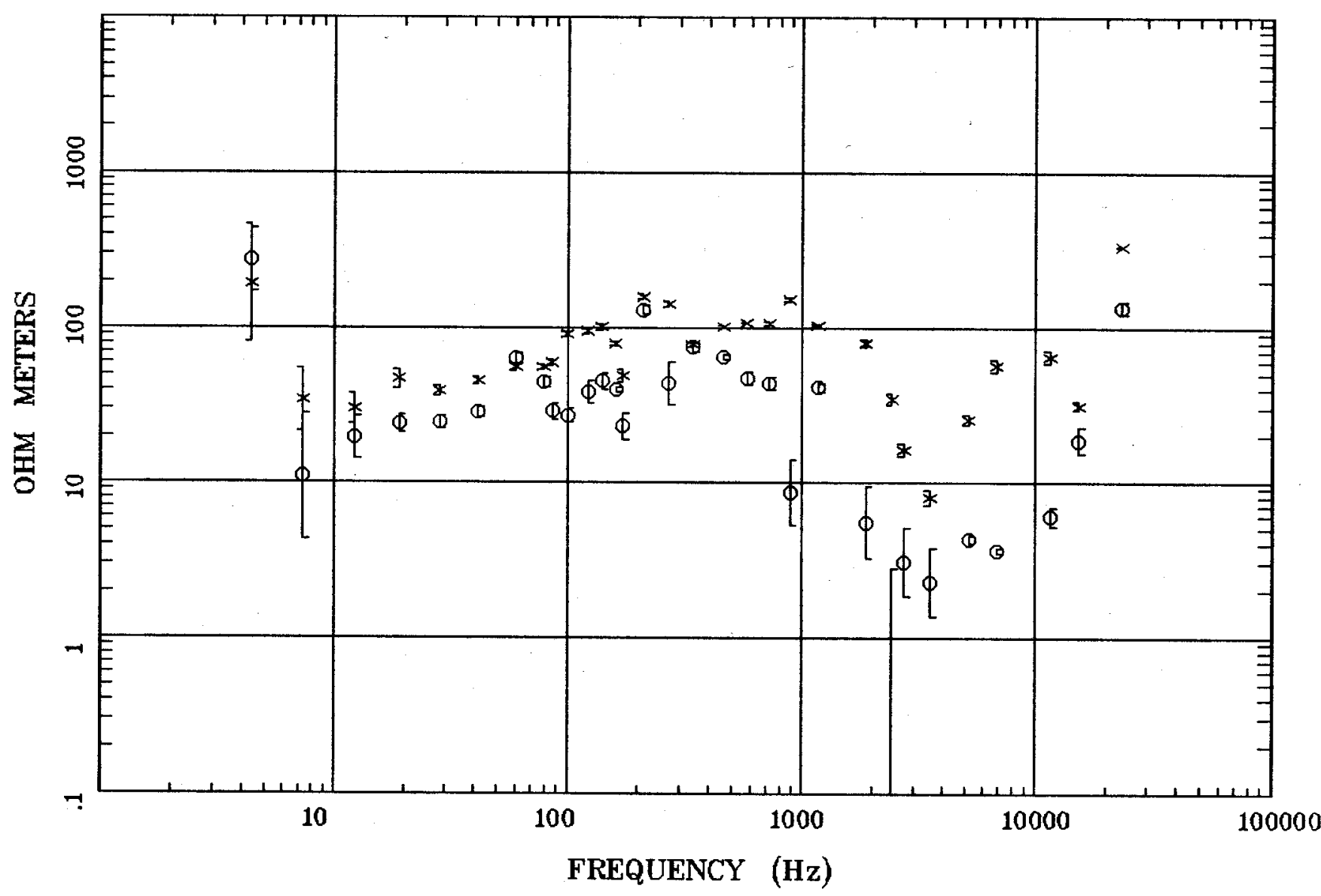

Client: City of Albuquerque Remote: $n$ Acquired: 13:5 May 10, 1998 Survey Co:USGS
Rotation:

Filename: rr16.avg

Channels: Ch1 Ch2 Ch3 Ch4 Ch5 Ch8 Ch9

Plotted: 15:34 Mar 08, 2001

<EMI - ElectroMagnetic Instruments > 
Station 16

IMPEDANCE PHASE

Albuquerque, NM

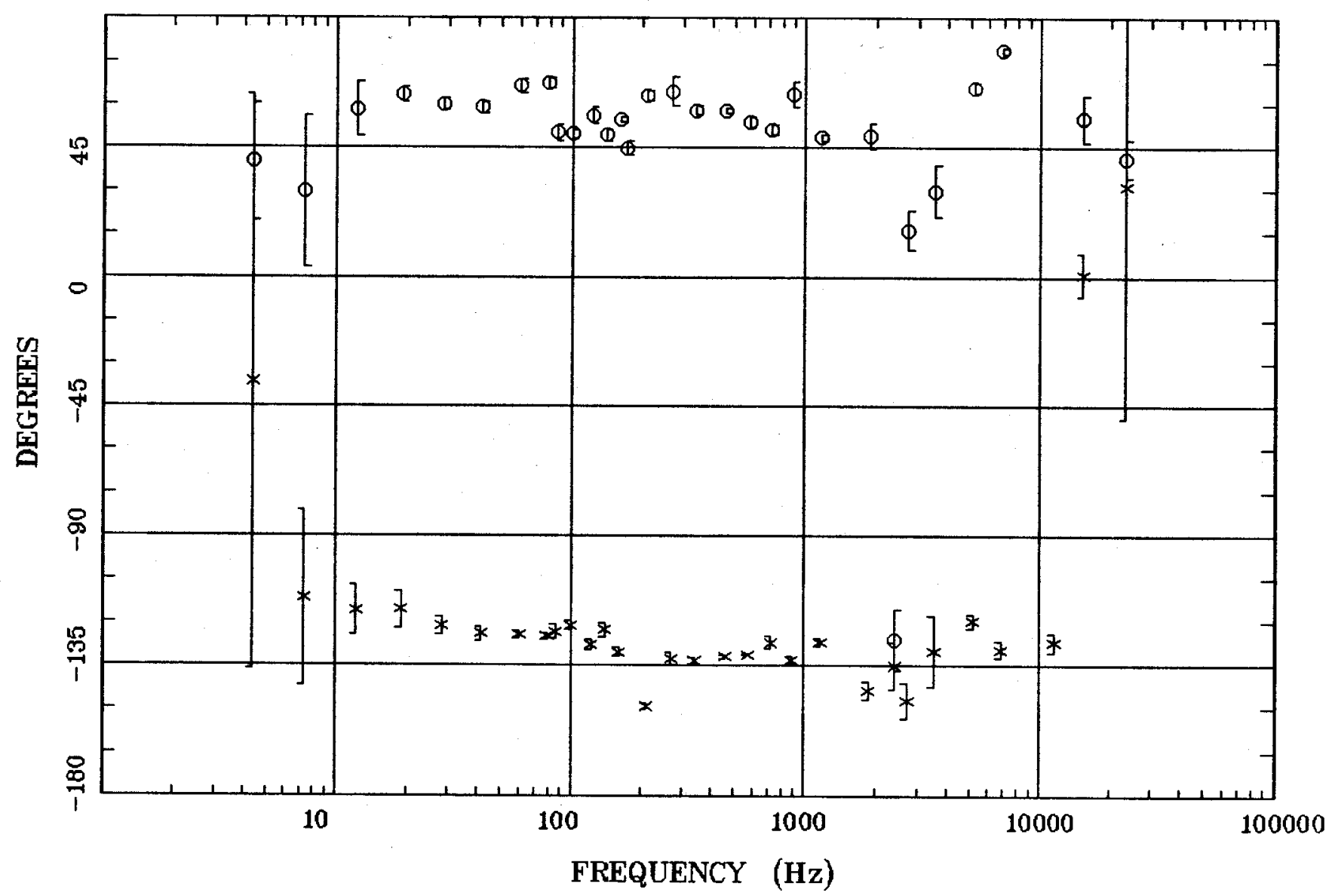

Client: City of Albuquerque Remote: $\mathbf{n}$

Rotation:

Acquired: 13:5 May 10, 1998

Filename: rr16.avg

Channels: Ch1 Ch2 Ch3 Ch4 Ch5 Ch8 Ch9 Survey Co:USGS

Platted: 15:34 Mar 08, 2001

< EMI - ElectroMagnetic Instruments > 


\section{Station 16}

ROTATION ANGLE

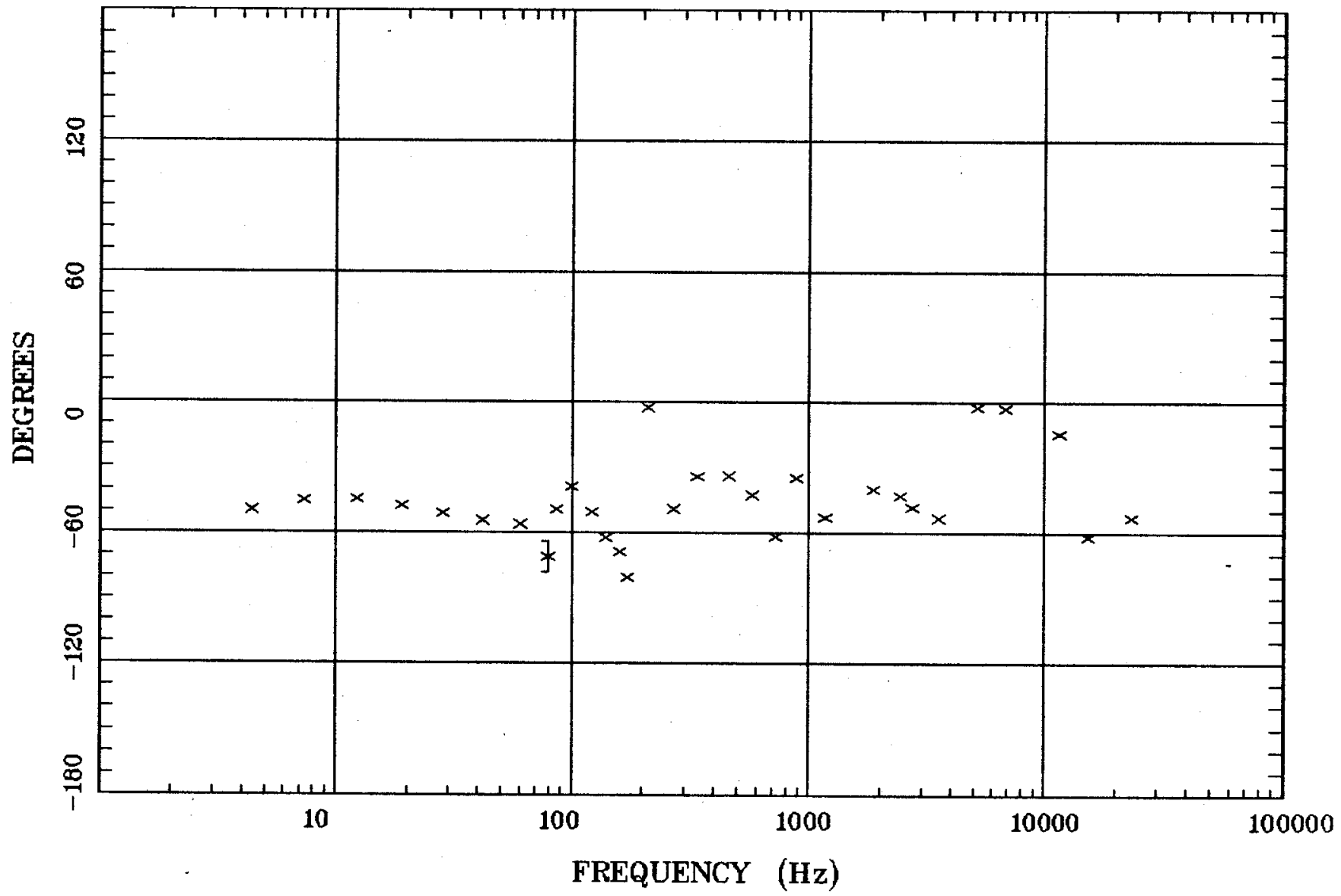

Client: City of Albuquerque Remote: $\mathbf{n}$

Acquired: 13:5 May 10, 1998 Survey Co:USGS
Albuquerque, NM

\section{Rotation:}

Filename: rr16.avg

Channels: Ch1 Ch2 Ch3 ch4 Ch5 Ch8 Ch9

Plotted: 15:34 Mar 08, 2001

$<$ EMI - ElectroMagnetic Instruments > 
Albuquerque, NM

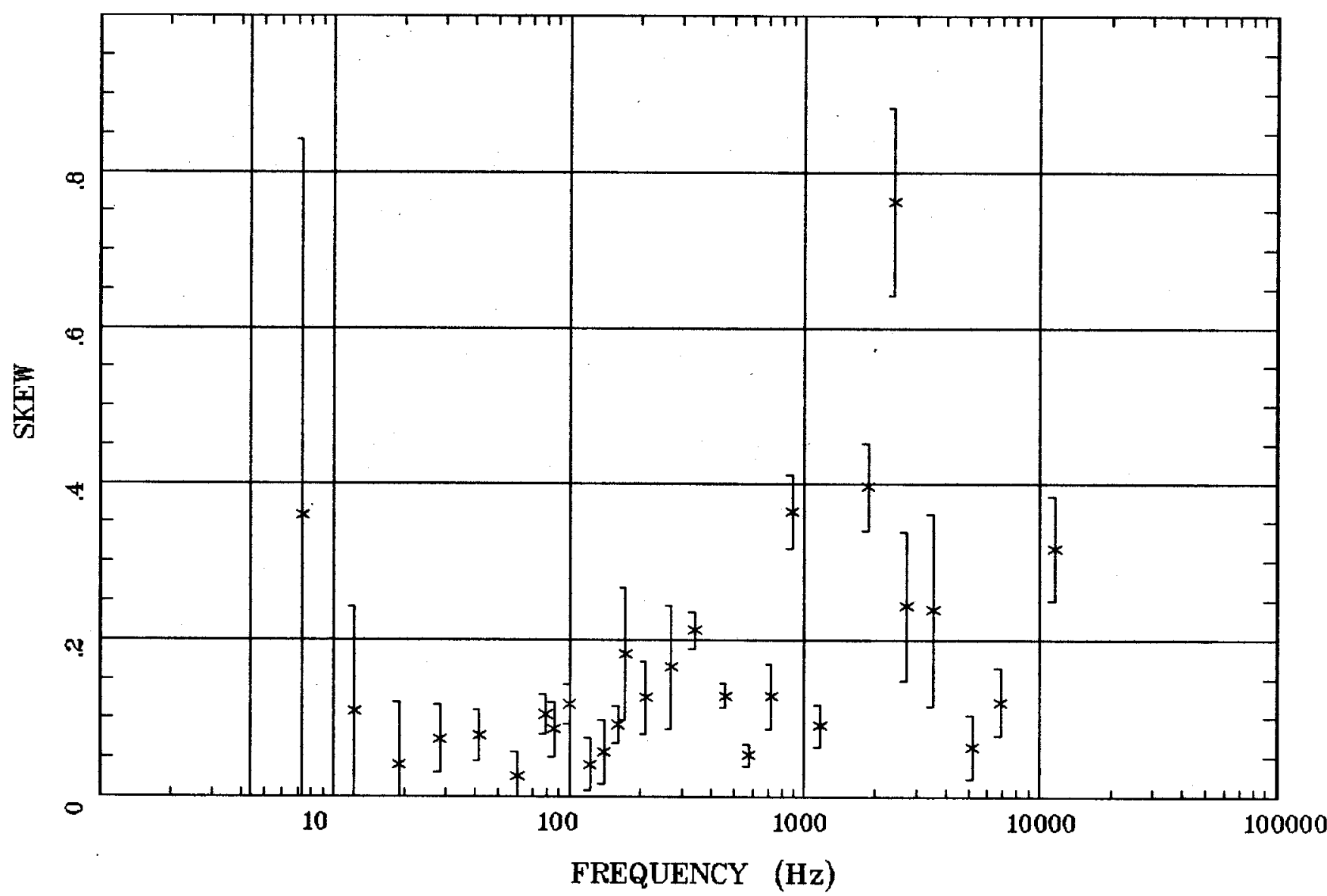

Client: City of Albuquerque Remote: $\mathbf{n}$

Acquired: 13:5 May 10, 1998 Survey Co:USGS
Rotation:

Filename: rr16.avg

Channels: Ch1 Ch2 Ch3 Ch4 Ch5 Ch8 Ch9 Plotted: 15:34 Mar 08, 2001

$<$ EMI - ElectroMagnetic Instruments 
E MULT Coh.

Albuquerque, NM

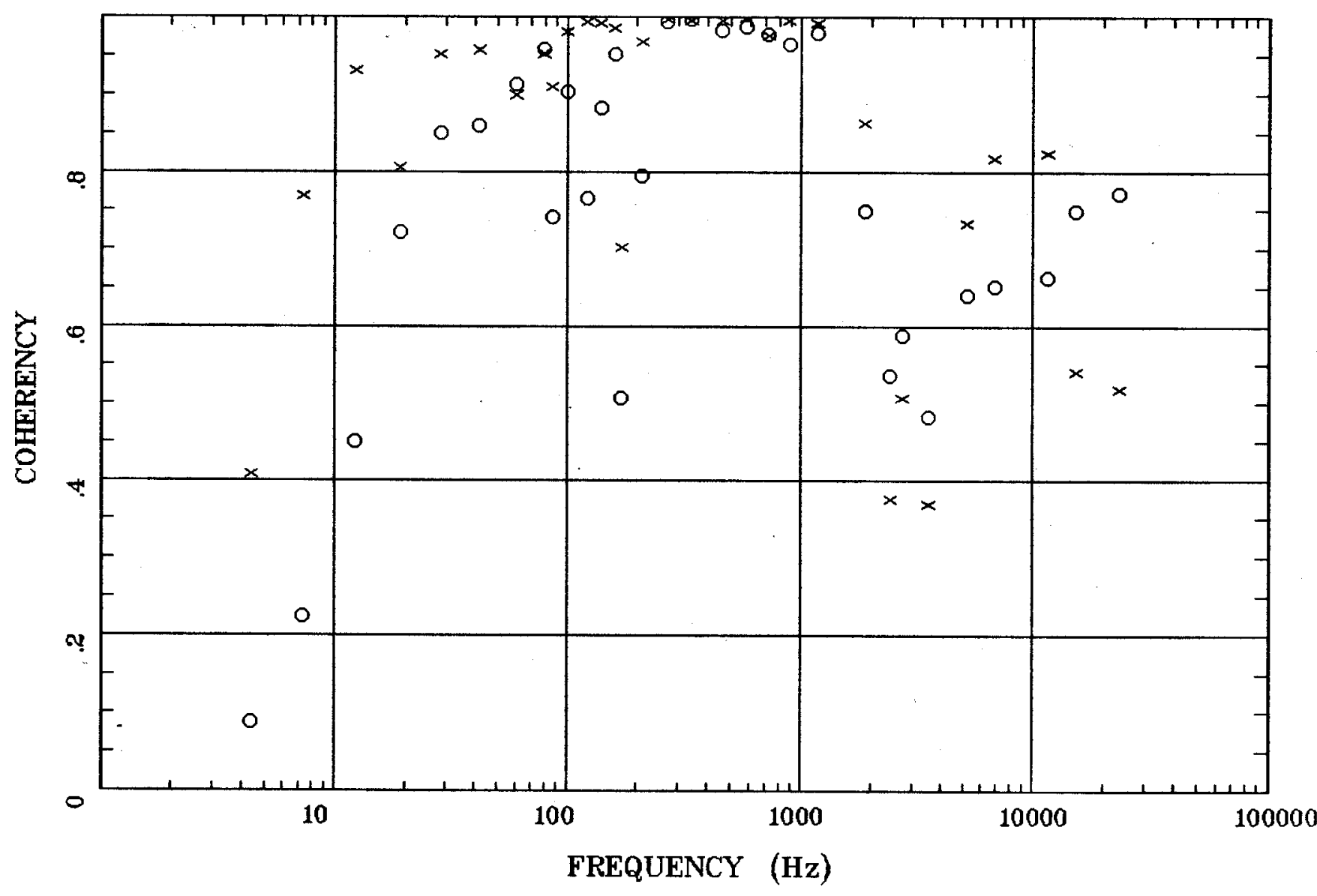

Client: City of Albuquerque Remote: $\mathbf{n}$

Acquired: 13:5 May 10, 1998 Survey Co:USGS
Rotation:

Filename: rr16.avg

Channels: Ch1 Ch2 Ch3 Ch4 Ch5 Ch8 Ch9

Plotted: 15:34 Mar 08, 2001

< EMI - ElectroMagnetic Instruments > 
Station 16

POLAR PLOTS

Albuquerque, NM

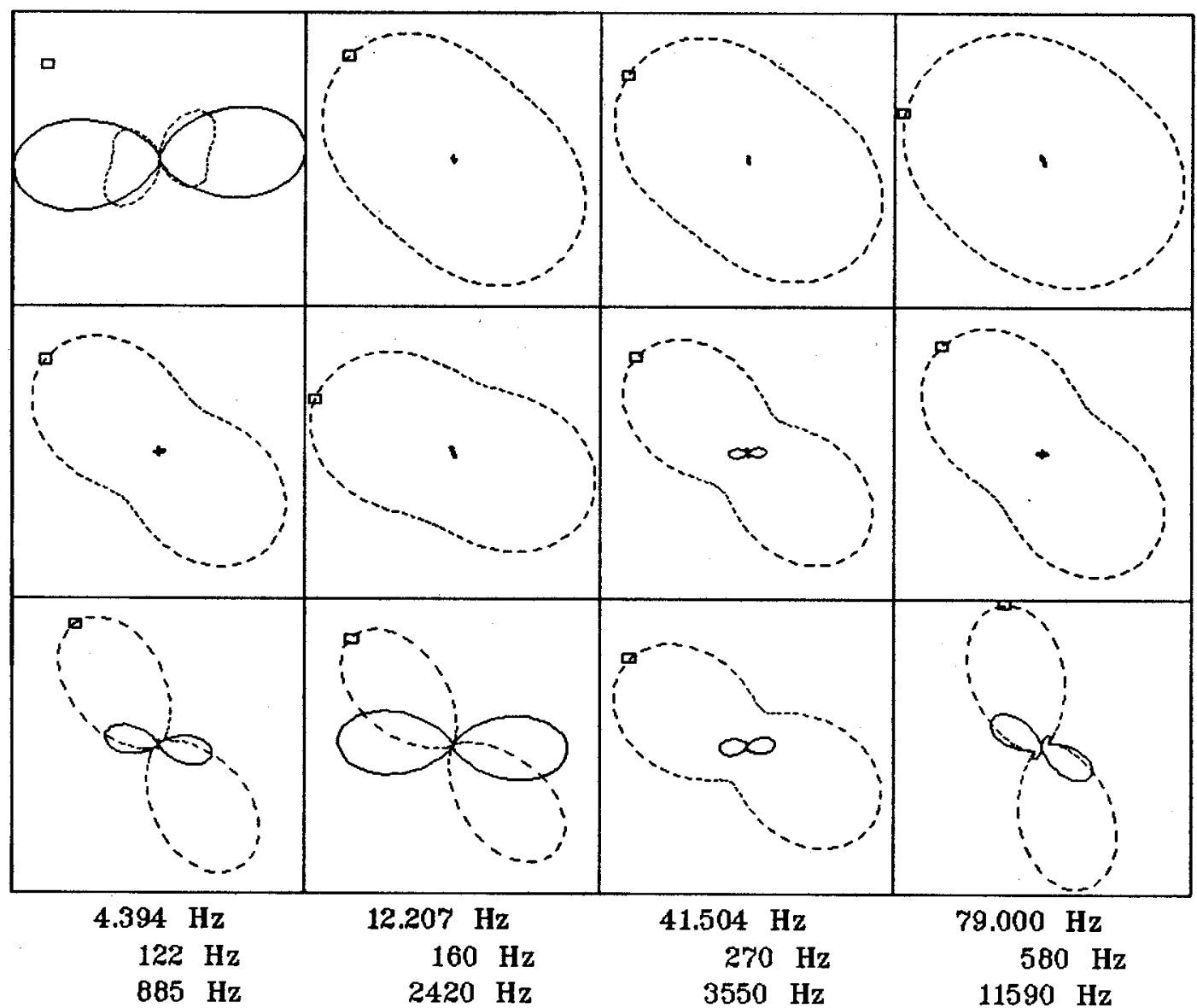

Client: City of Albuquerque

Rotation:

Remote: $\mathbf{n}$

Acquired: 13:5 May 10, 1998

Filename: rr16.avg

Channels: Ch1 Ch2 Ch3 Ch4 Ch5 Ch8 Ch9

Plotted: 15:34 Mar 08, 2001

Survey Co:USGS

< EMI - ElectroMagnetic Instruments > 


\section{Station 16}

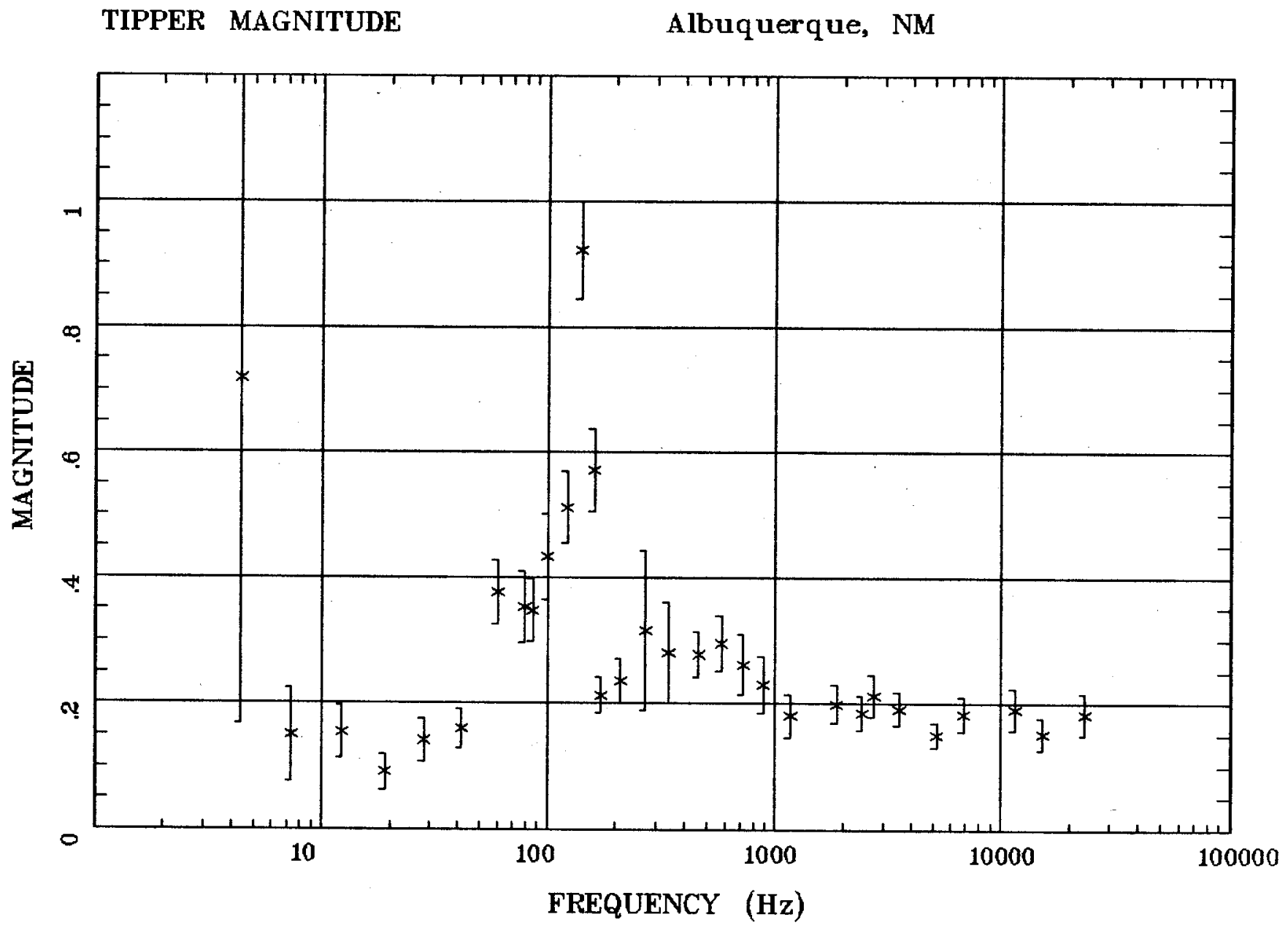

Client: City of Albuquerque Remote: $\mathbf{n}$

Acquired: 13:5 May 10, 1998 Survey Co:USGS
Rotation:

Filename: rr16.avg

Channels: Ch1 Ch2 Ch3 Ch4 Ch5 Ch8 Ch9

Plotted: 15:34 Mar 08, 2001

$<$ EMI - ElectroMagnetic Instruments 
TIPPER STRIKE

Albuquerque, NM

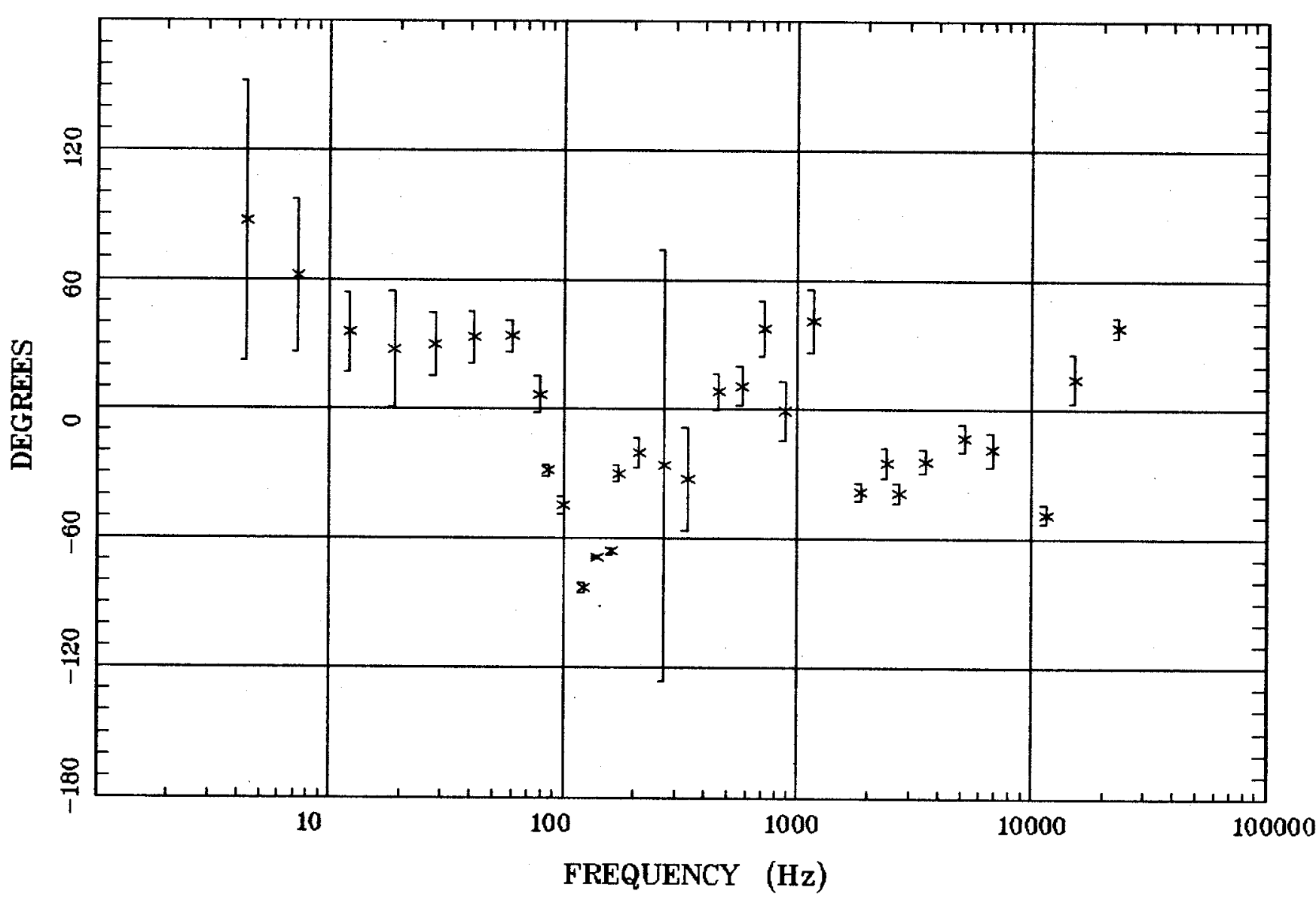

Client: City of Albuquerque Remote: $\mathbf{n}$

Acquired: 13:5 May 10, 1998 Survey Co:USGS

\section{Rotation:}

Filename: rr16.avg

Channels: Ch1 Ch2 Ch3 Ch4 Ch5 Ch8 Ch9

Plotted: 15:34 Mar 08, 2001

< EMI - ElectroMagnetic Instruments > 


\section{Station 16}

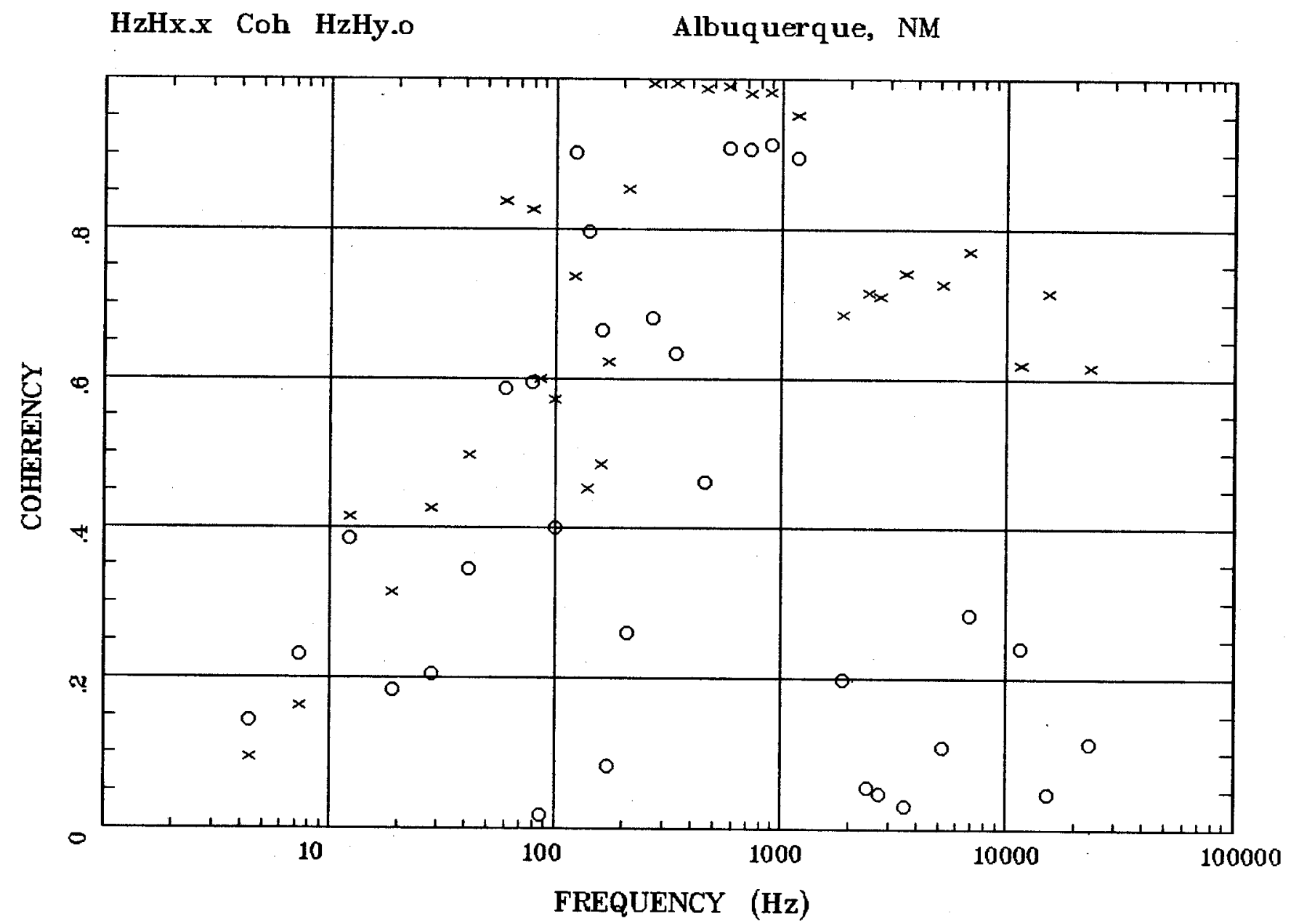

Client: City of Albuquerque

Remote: $\mathbf{n}$

Acquired: 13:5 May 10, 1998 Survey Co:USGS
Rotation:

Filename: rr16.avg

Channels: Ch1 Ch2 Ch3 Ch4 Ch5 Ch8 Ch9

Plotted: 15:34 Mar 08, 2001

$<$ EMI - ElectroMagnetic Instruments 


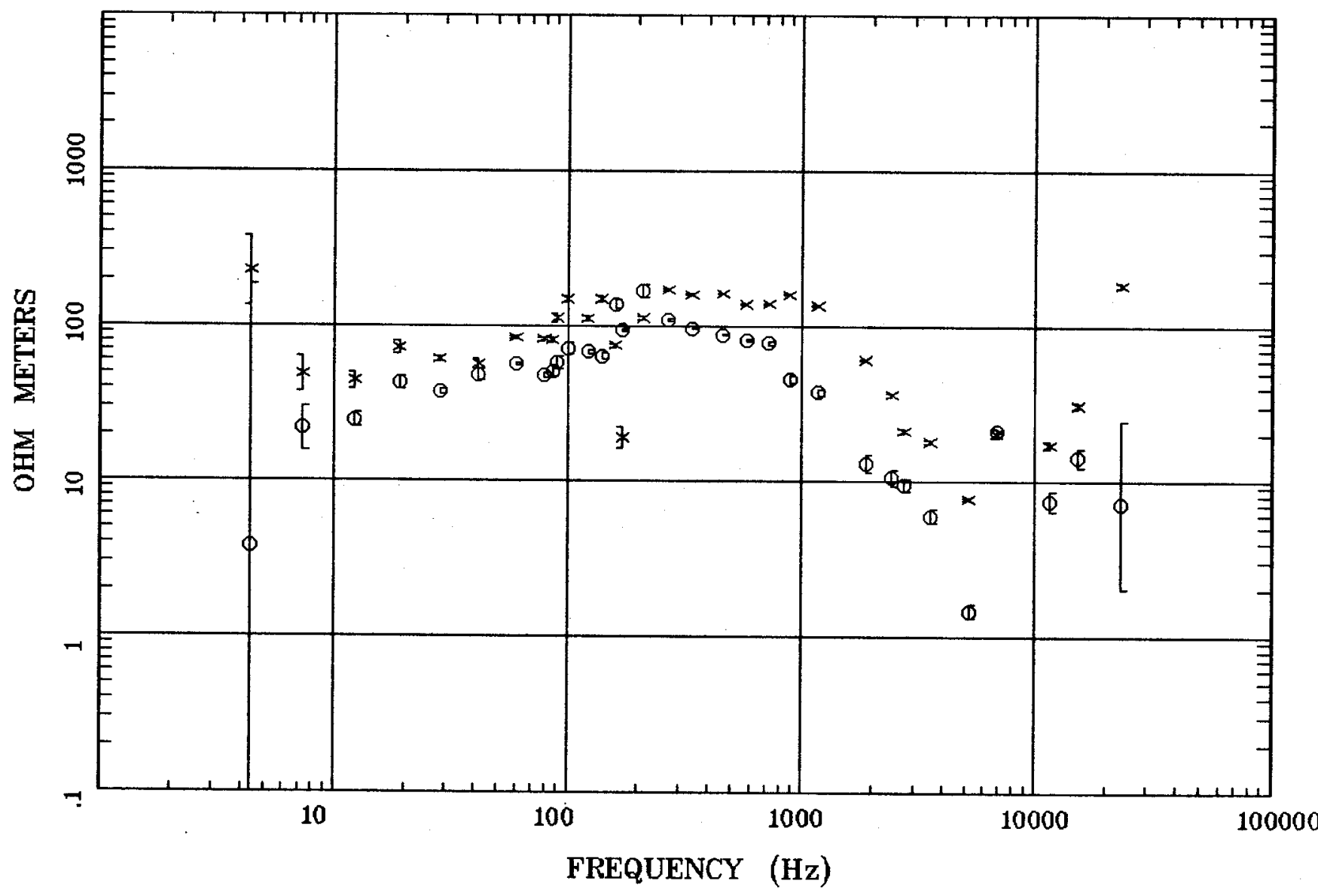

Client: City of Albuquerque Remote: $\mathbf{n}$

Acquired: 10:2 May 10, 1998 Survey Co:USGS
Rotation:

Filename: rr15all avg

Channels: Ch1 Ch2 Ch3 Ch4 Ch5 Ch8 Ch9 Plotted: 15:35 Mar 08, 2001

< EMI - ElectroMagnetic Instruments > 
Station 15

IMPEDANCE PHASE

Albuquerque, NM

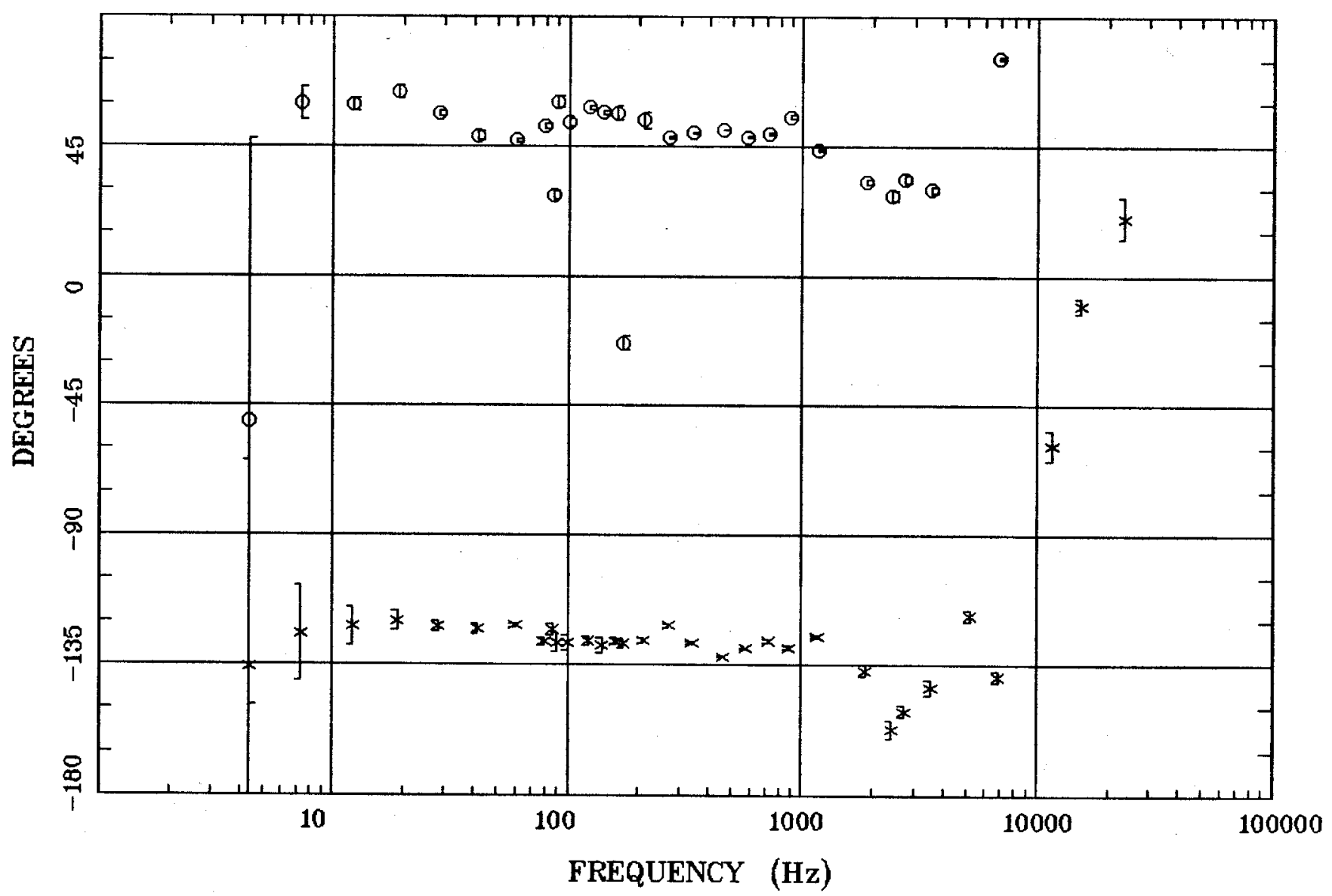

Client: City of Albuquerque Remote: $\mathbf{n}$

Acquired: 10:2 May 10, 1998

Survey Co:USGS
Rotation:

Filename: rr15all.avg

Channels: Ch1 Ch2 Ch3 Ch4 Ch5 Ch8 Ch9

Platted: 15:35 Mar 08, 2001

< EMI - ElectroMagnetic Instruments 
ROTATION ANGLE

Albuquerque, NM

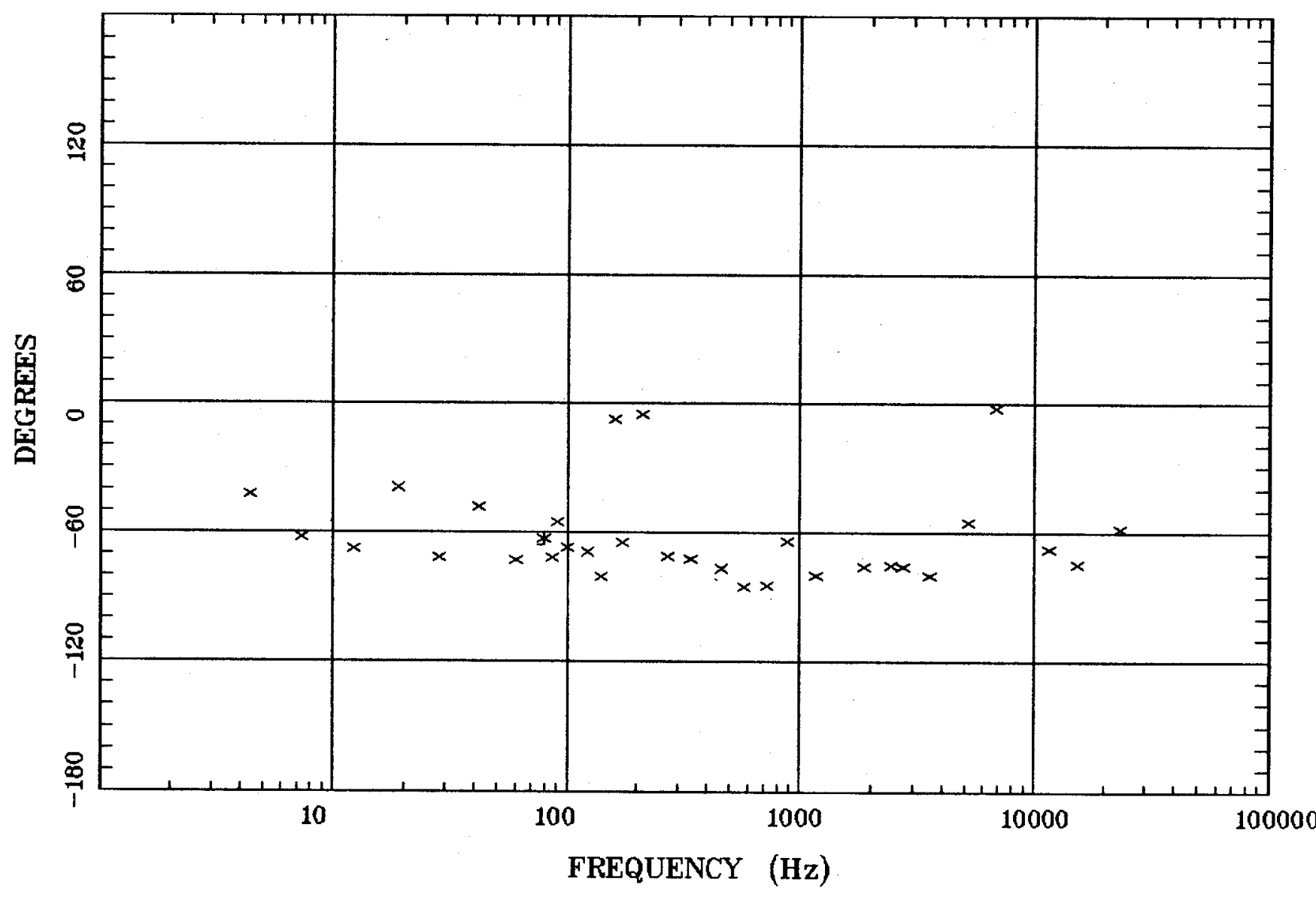

Client: City of Albuquerque Remote: $\mathrm{n}$

Acquired: 10:2 May 10, 1998 Survey Co:USGS
Rotation:

Filename: rr15all.avg

Channels: Ch1 Ch2 Ch3 Ch4 Ch5 Ch8 Ch9

Plotted: 15:35 Mar 08, 2001

<EMI - ElectroMagnetic Instruments > 
Albuquerque, NM

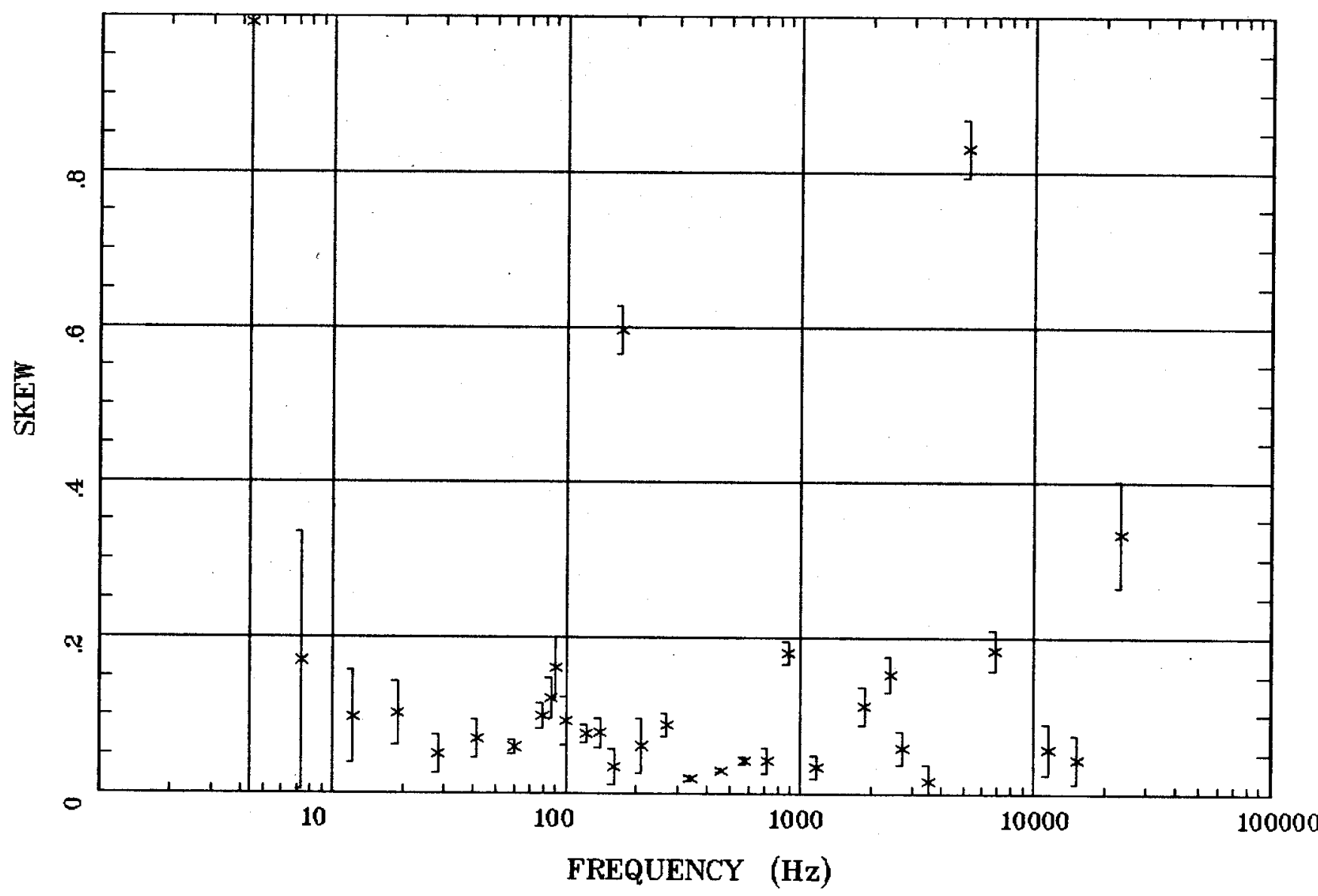

Client: City of Albuquerque Remote: $\mathbf{n}$

Acquired: 10:2 May 10, 1998 Survey Co:USGS
Rotation:

Filename: rr15all.avg

Channels: Ch1 Ch2 Ch3 Ch4 Ch5 Ch8 Ch9

Plotted: 15:35 Mar 08, 2001

$<$ EMI - ElectroMagnetic Instruments > 
E MULT Coh.

Albuquerque, NM

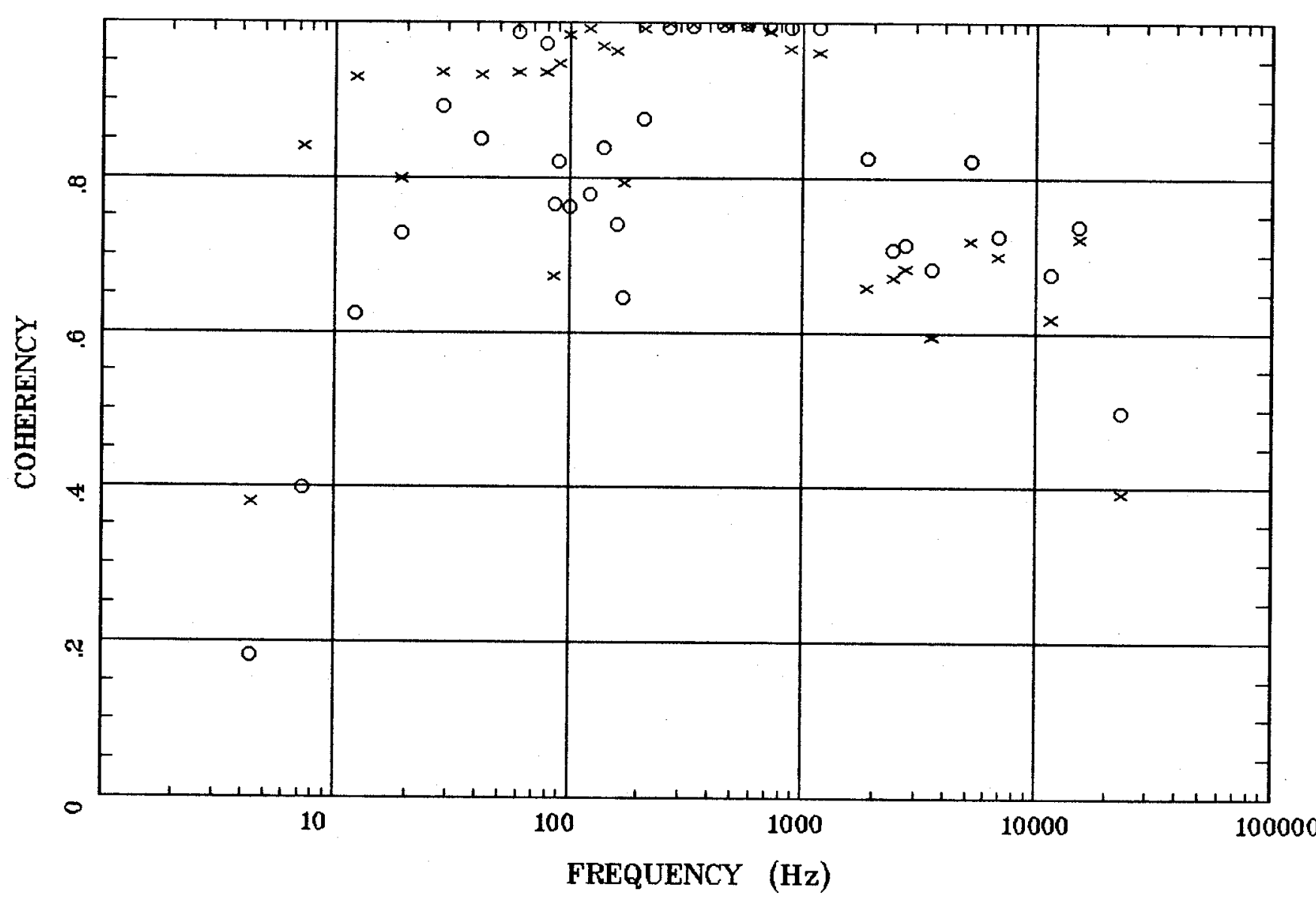

Client: City of Albuquerque Remote: $\mathbf{n}$

Acquired: 10:2 May 10, 1998 Survey Co:USGS
Rotation:

Filename: rr15all.avg

Channels: Ch1 Ch2 Ch3 Ch4 Ch5 Ch8 Ch9 Plotted: 15:35 Mar 08, 2001

$<$ EMI - ElectroMagnetic Instruments > 
Station 15

POLAR PLOTS

Albuquerque, NM

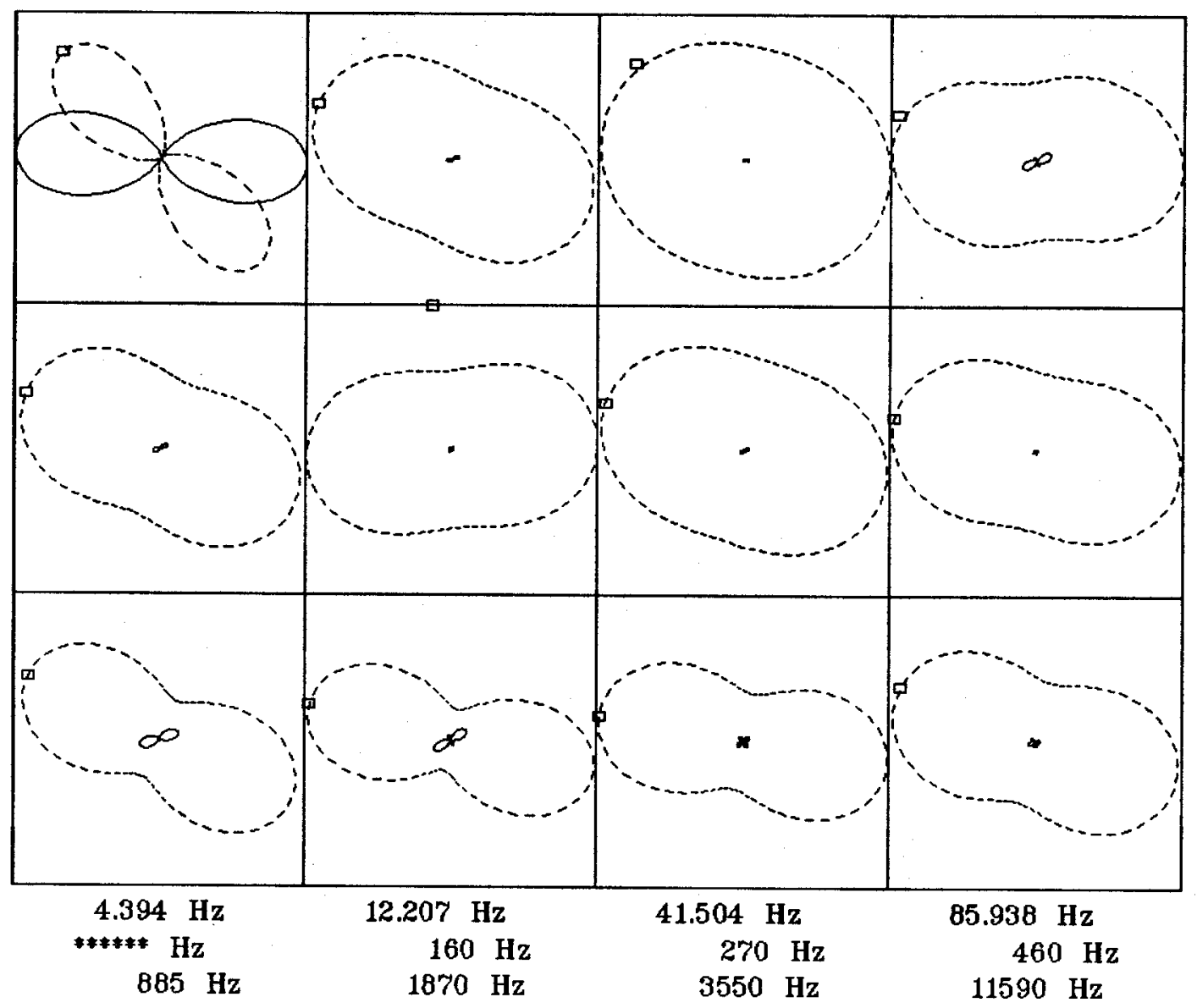

Client: City of Albuquerque

Rotation:

Filename: rr15all.avg

Channels: Ch1 Ch2 Ch3 Ch4 Ch5 Ch8 Ch9

Platted: 15:35 Mar 08, 2001

Acquired: 10:2 May 10, 1998

$<$ EMI - ElectroMagnetic Instruments 


\section{Station 15}

TIPPER MAGNITUDE

Albuquerque, NM

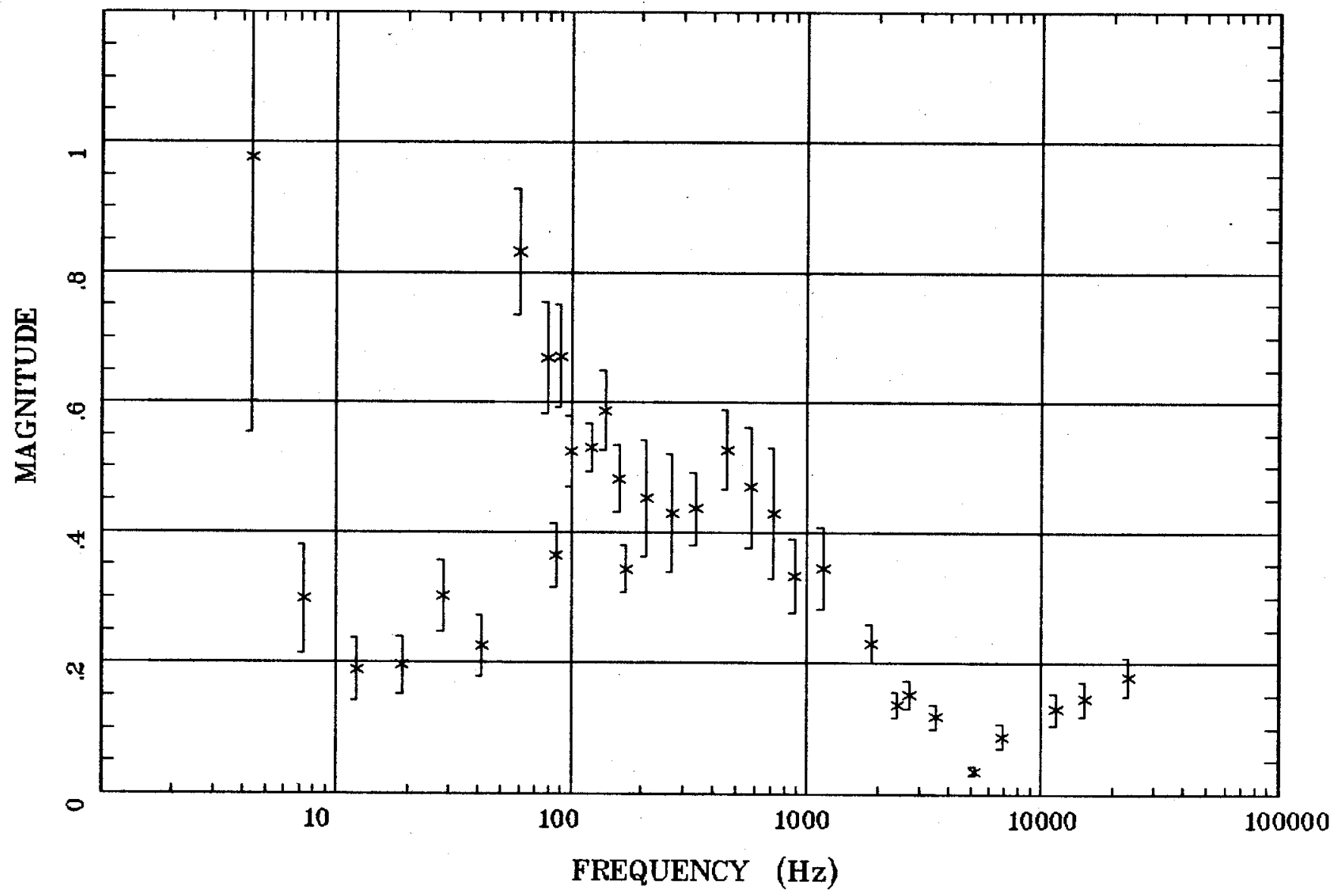

Client: City of Albuquerque

Remote: $\mathbf{n}$

Acquired: 10:2 May 10, 1998

Survey Co:USGS
Rotation:

Filename: rr15all.avg

Channels: Ch1 Ch2 Ch3 Ch4 Ch5 Ch8 Ch9

Plotted: 15:35 Mar 08, 2001

< EMI - ElectroMagnetic Instruments 


\section{Station 15}

TIPPER STRIKE

Albuquerque, NM

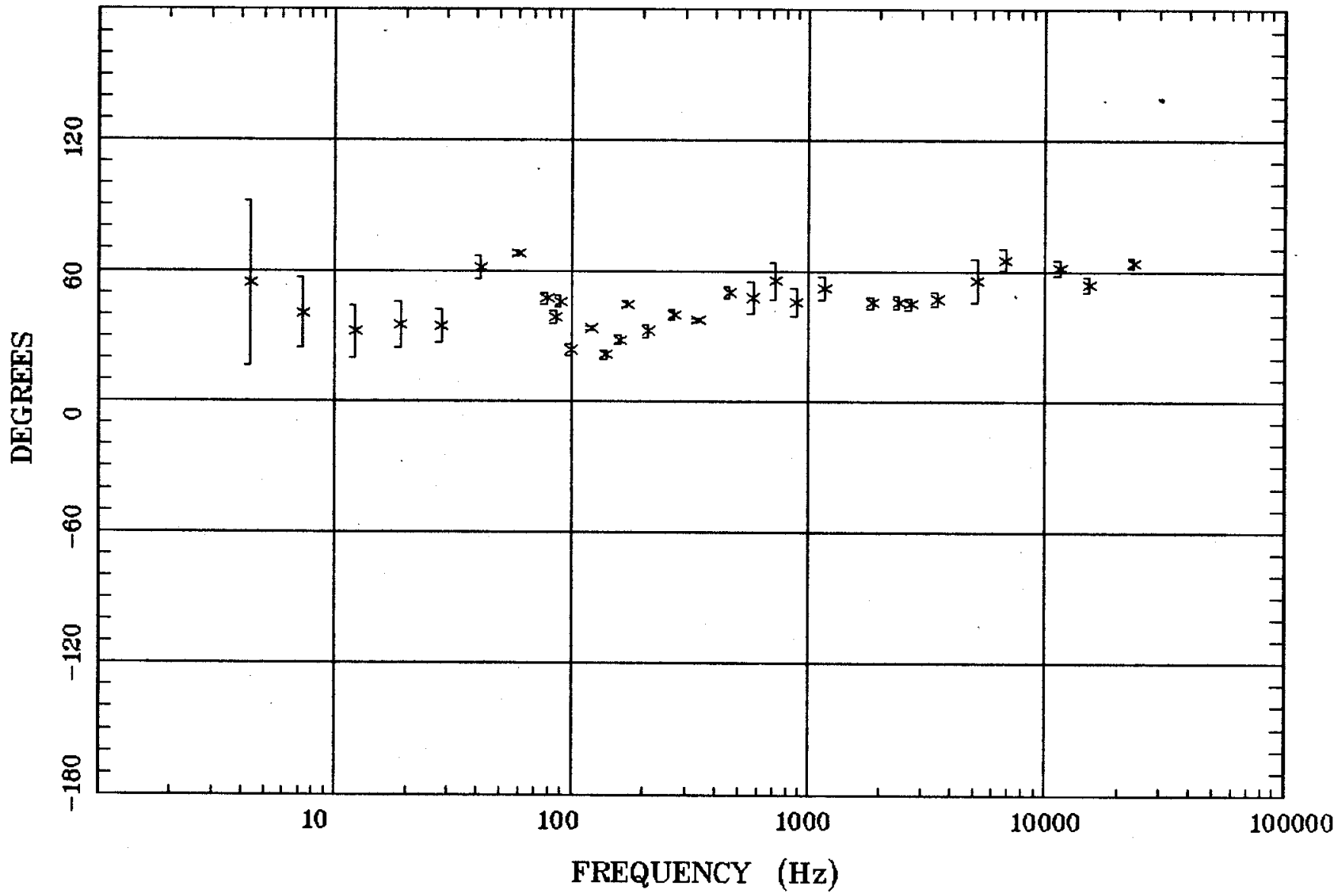

Client: City of Albuquerque Remote: $\mathrm{n}$

Acquired: 10:2 May 10, 1998

Survey Co:USGS
Rotation:

Filename: rr15all.avg

Channels: Ch1 Ch2 Ch3 Ch4 Ch5 Ch8 Ch9

Plotted: 15:35 Mar 08, 2001

$<$ EMI - ElectroMagnetic Instruments 


\section{Station 15}

HzHx.x Coh HzHy.o Albuquerque, NM

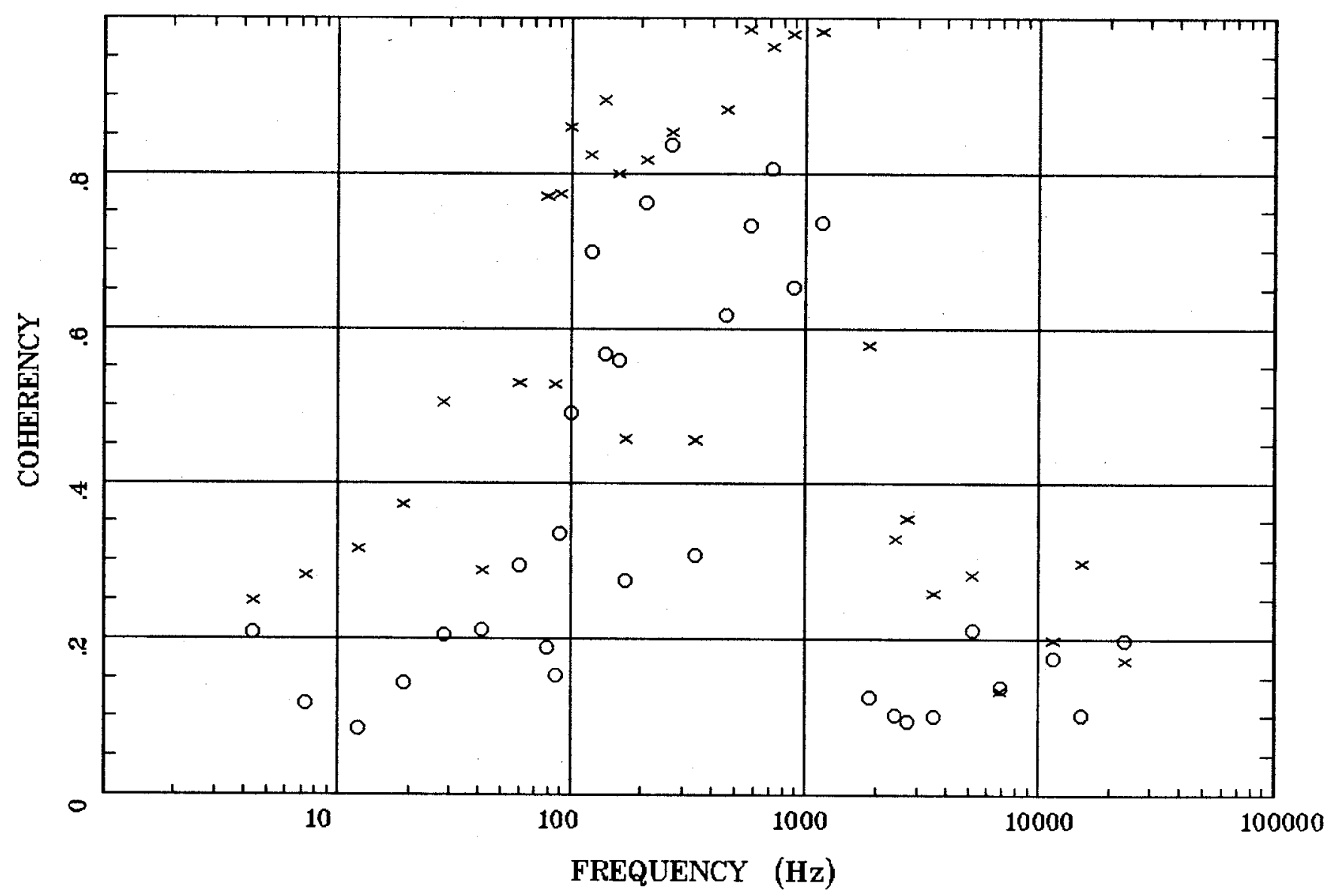

Client: City of Albuquerque Remote: $\mathrm{n}$

Acquired: 10:2 May 10, 1998 Survey Co:USGS
Rotation:

Filename: rr15all.avg

Channels: Ch1 Ch2 Ch3 Ch4 Ch5 Ch8 Ch9

Plotted: 15:35 Mar 08, 2001

$<$ EMI - ElectroMagnetic Instruments 


\section{Station 5}

APPARENT RESISTIVITY

NM, Albuquerque, Volcanoes

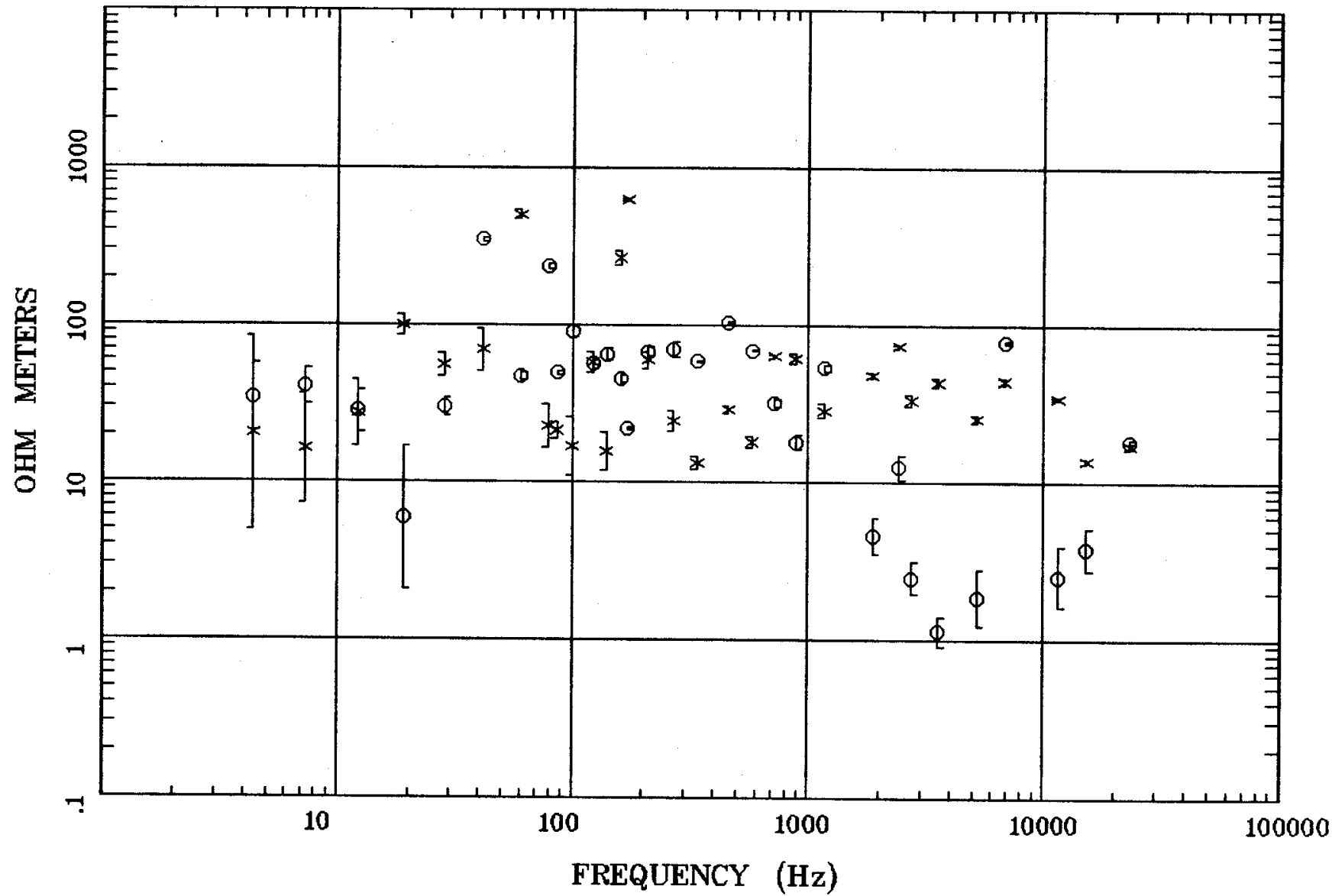

Client:

Remote: Local B

Acquired: 14:1 Aug 23, 1997

Survey Co:USGS GD-MRP Denver
Rotation:

Filename: al05.ave

Channels: Ch1 Ch2 Ch3 Ch4 Ch5 Ch8 Ch9

Plotted: 15:29 Mar 08, 2001

< EMI - ElectroMagnetic Instruments > 
IMPEDANCE PHASE

NM, Albuquerque, Volcanoes

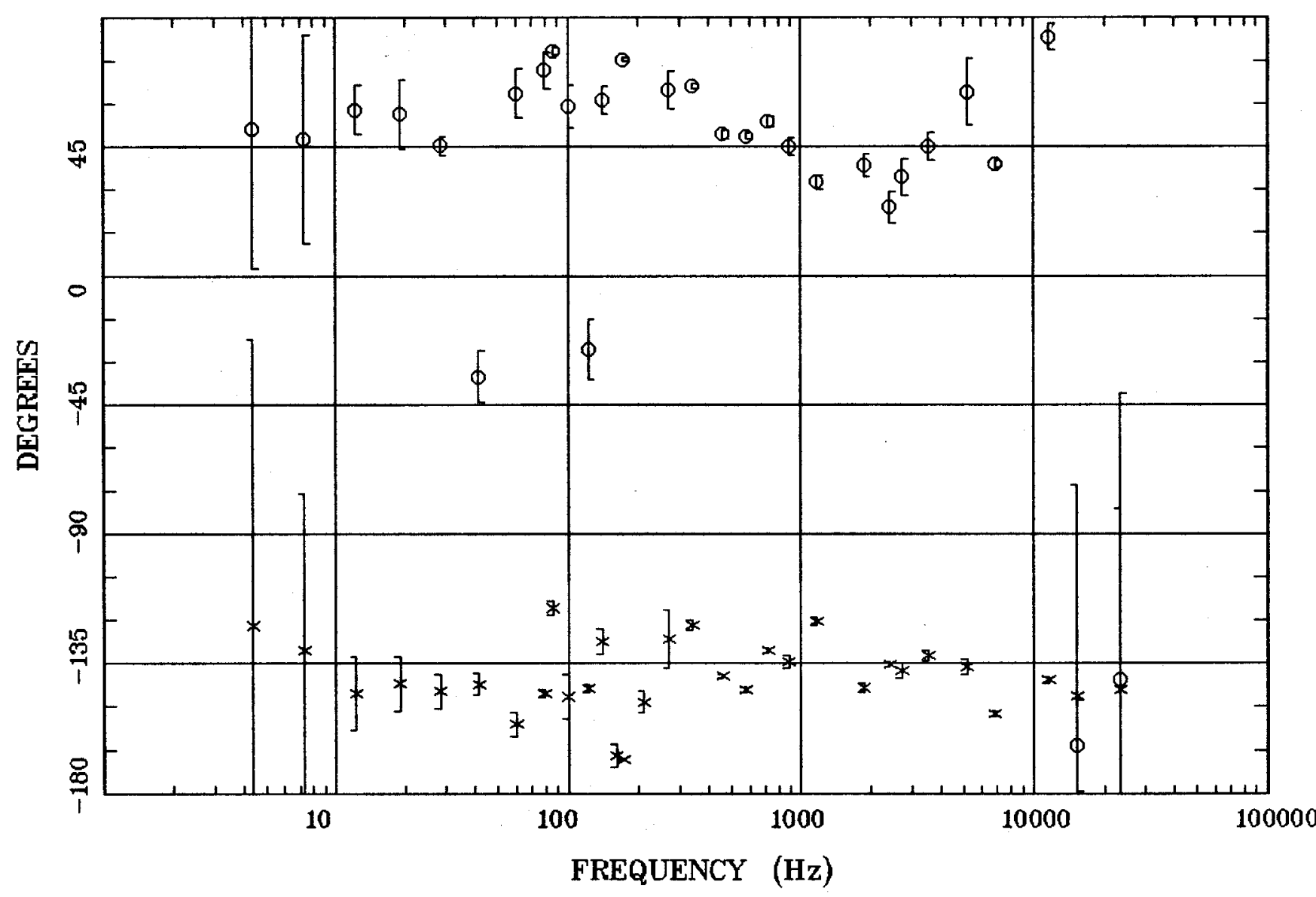

Client:

Remote: Local B

Acquired: 14:1 Aug 23, 1997

Survey Co:USGS GD-MRP Denver
Rotation:

Filename: al05.ave

Channels: Ch1 Ch2 Ch3 Ch4 Ch5 Ch8 Ch9

Plotted: 15:29 Mar 08, 2001

<EMI - ElectroMagnetic Instruments > 


\section{Station 5}

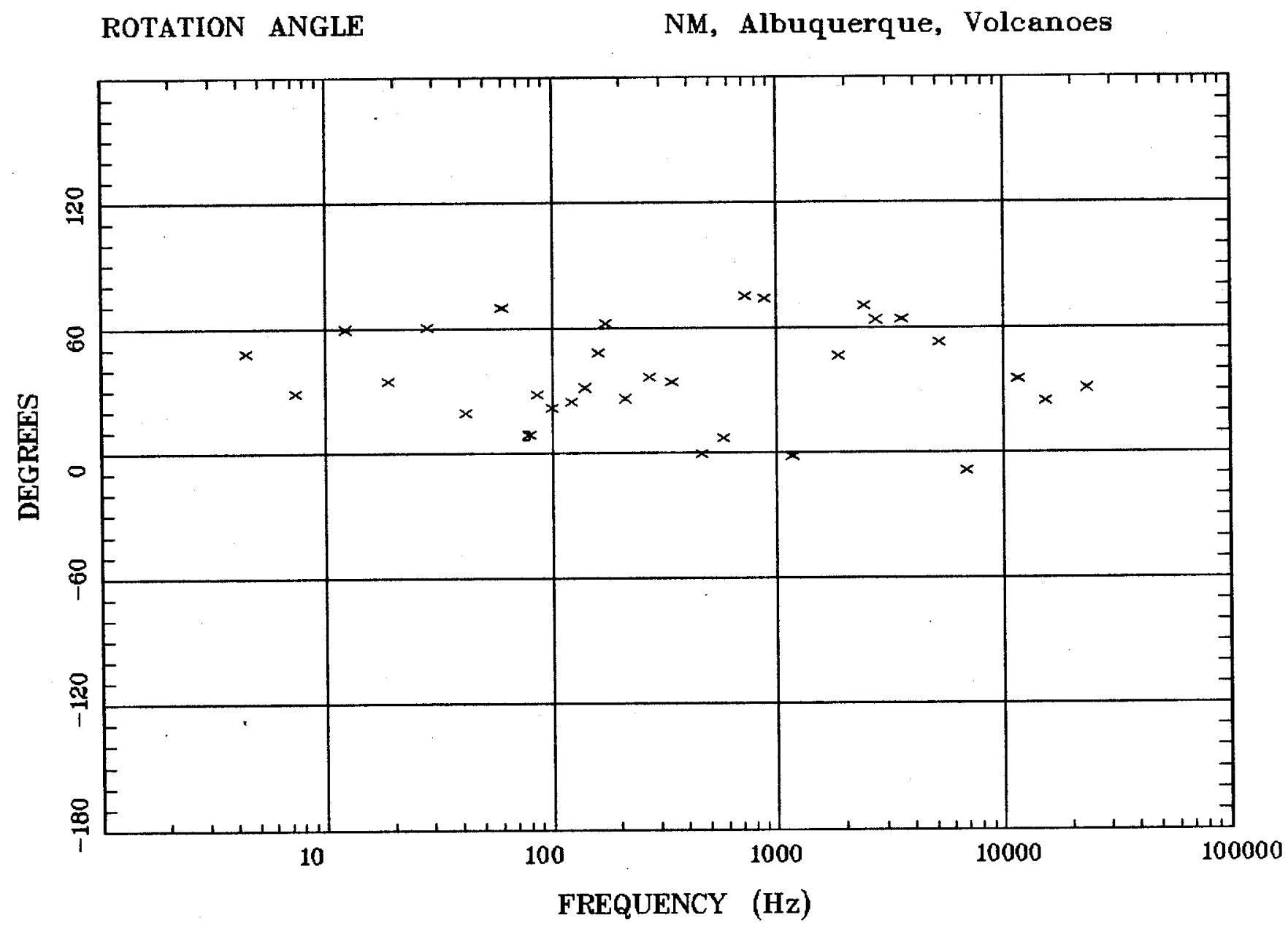

Client:

Remote: Local B

Acquired: 14:1 Aug 23, 1997 Survey Co:USGS GD-MRP Denver
Rotation:

Filename: al05.ave

Channels: Ch1 Ch2 Ch3 Ch4 Ch5 Ch8 Ch9

Plotted: 15:29 Mar 08, 2001

< EMI - ElectroMagnetic Instruments > 


\section{Station 5}

IMPEDANCE SKEW

NM, Albuquerque, Volcanoes

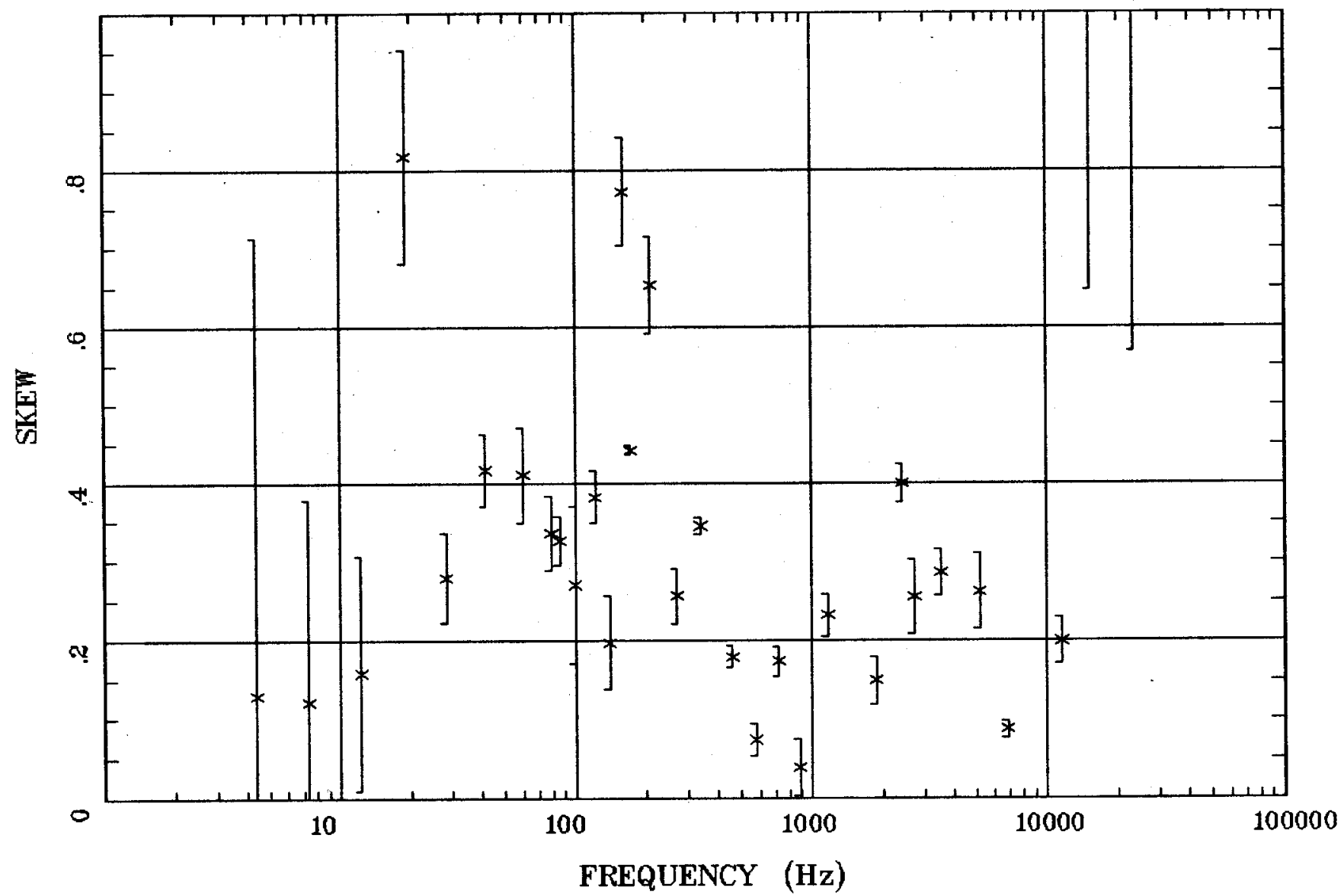

Client:

Remote: Local $B$

Acquired: 14:1 Aug 23, 1997

Survey Co:USGS GD-MRP Denver
Rotation:

Filename: al05.ave

Channels: Ch1 Ch2 Ch3 Ch4 Ch5 Ch8 Ch9

Plotted: 15:29 Mar 08, 2001

< EMI - ElectroMagnetic Instruments 


\section{Station 5}

E MULT Coh.

NM, Albuquerque, Volcanoes

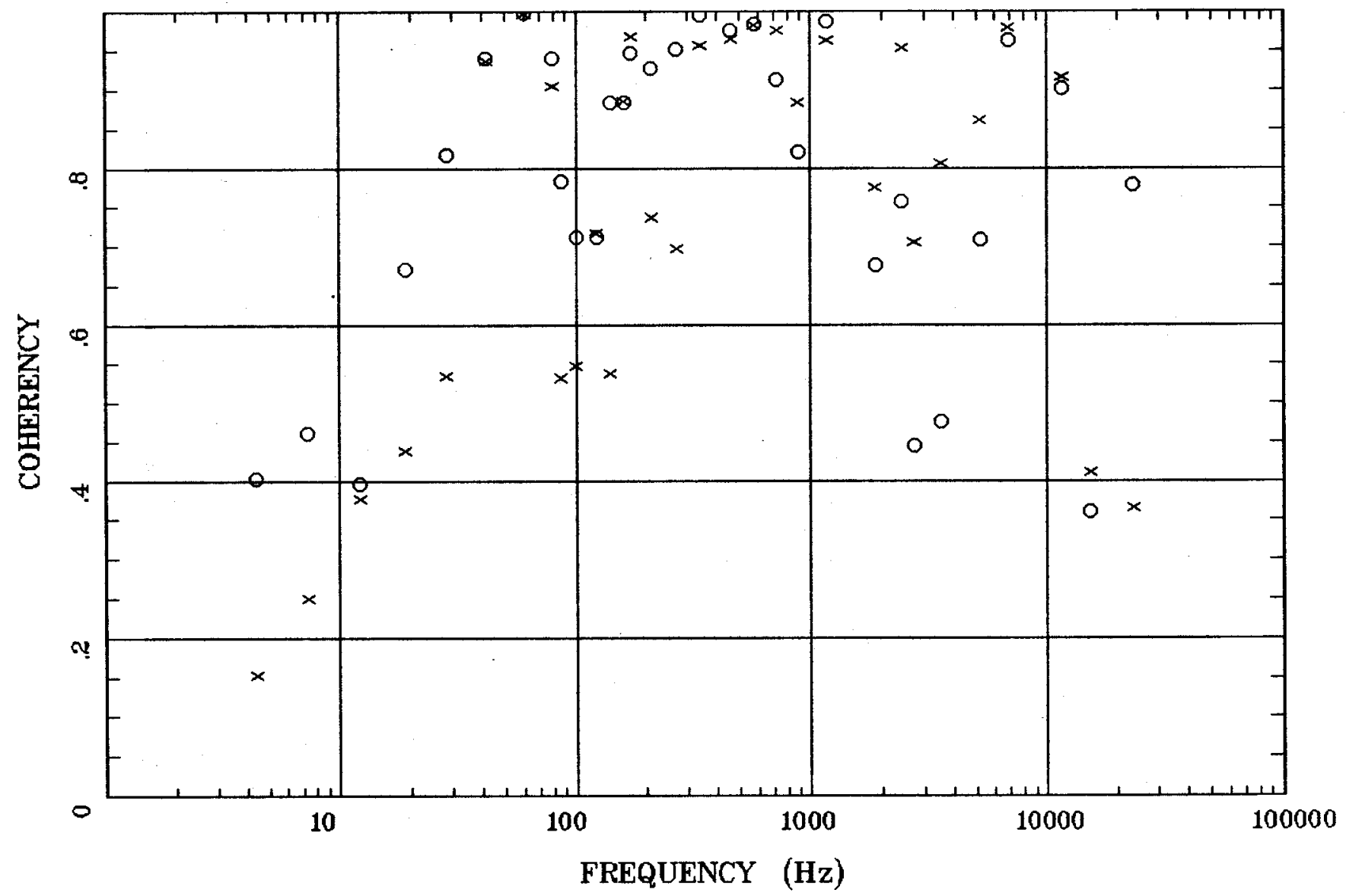

Client:

Remote: Local B

Acquired: 14:1 Aug 23, 1997

Survey Co:USGS GD-MRP Denver
Rotation:

Filename: al05.ave

Channels: Ch1 Ch2 Ch3 Ch4 Ch5 Ch8 Ch9

Plotted: 15:29 Mar 08, 2001

< EMI - ElectroMagnetic Instruments > 


\section{Station 5}

POLAR PLOTS

NM, Albuquerque, Volcanoes

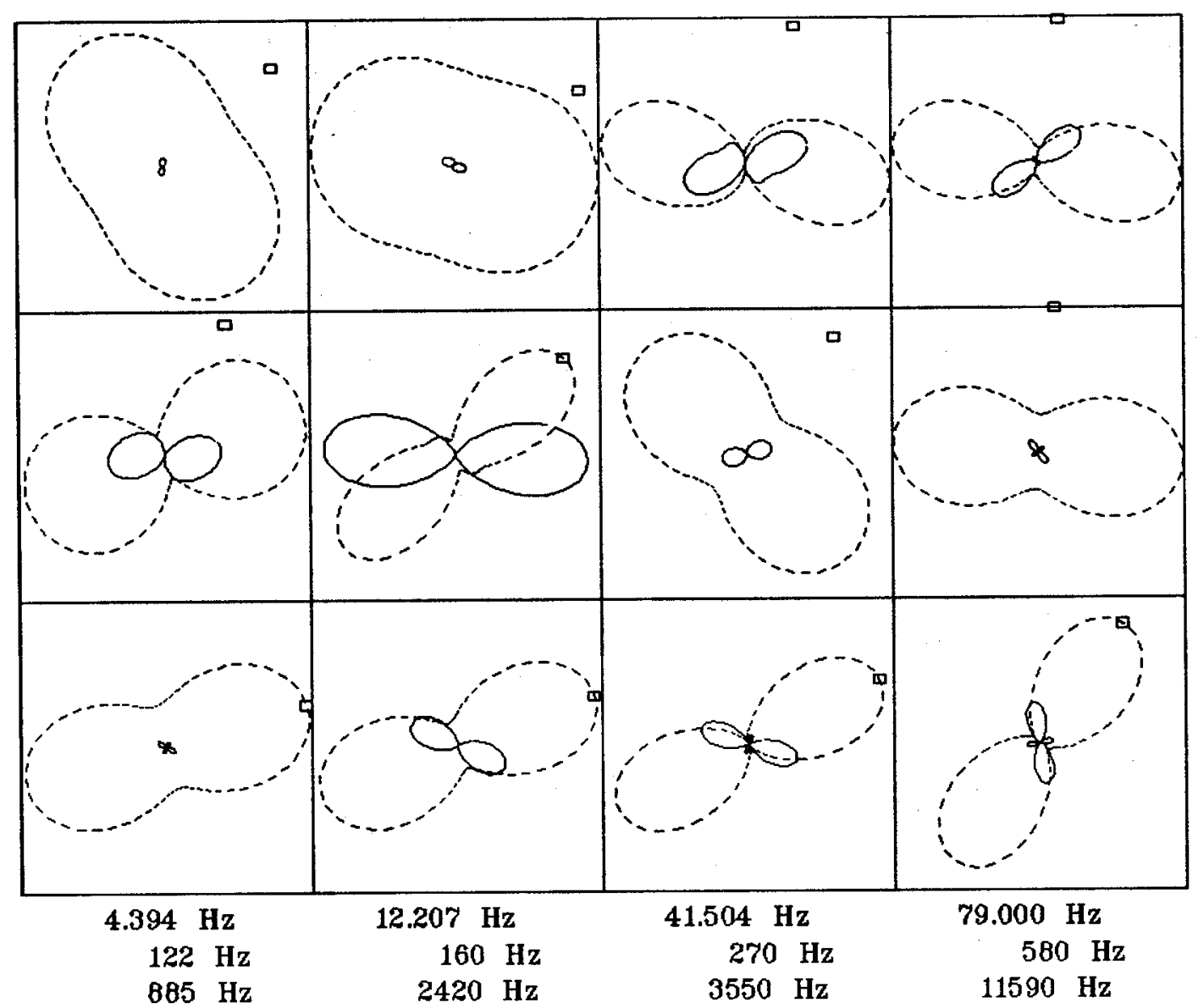

Client:

Remote: Local B

Acquired: 14:1 Aug 23, 1997

Survey Co:USGS GD-MRP Denver

Rotation:

Filename: al05.ave

Channels: Ch1 Ch2 Ch3 Ch4 Ch5 Ch8 Ch9

Plotted: 15:29 Mar 08, 2001

< EMI - ElectroMagnetic Instruments 


\section{Station 5}

TIPPER MAGNITUDE

NM, Albuquerque, Volcanoes

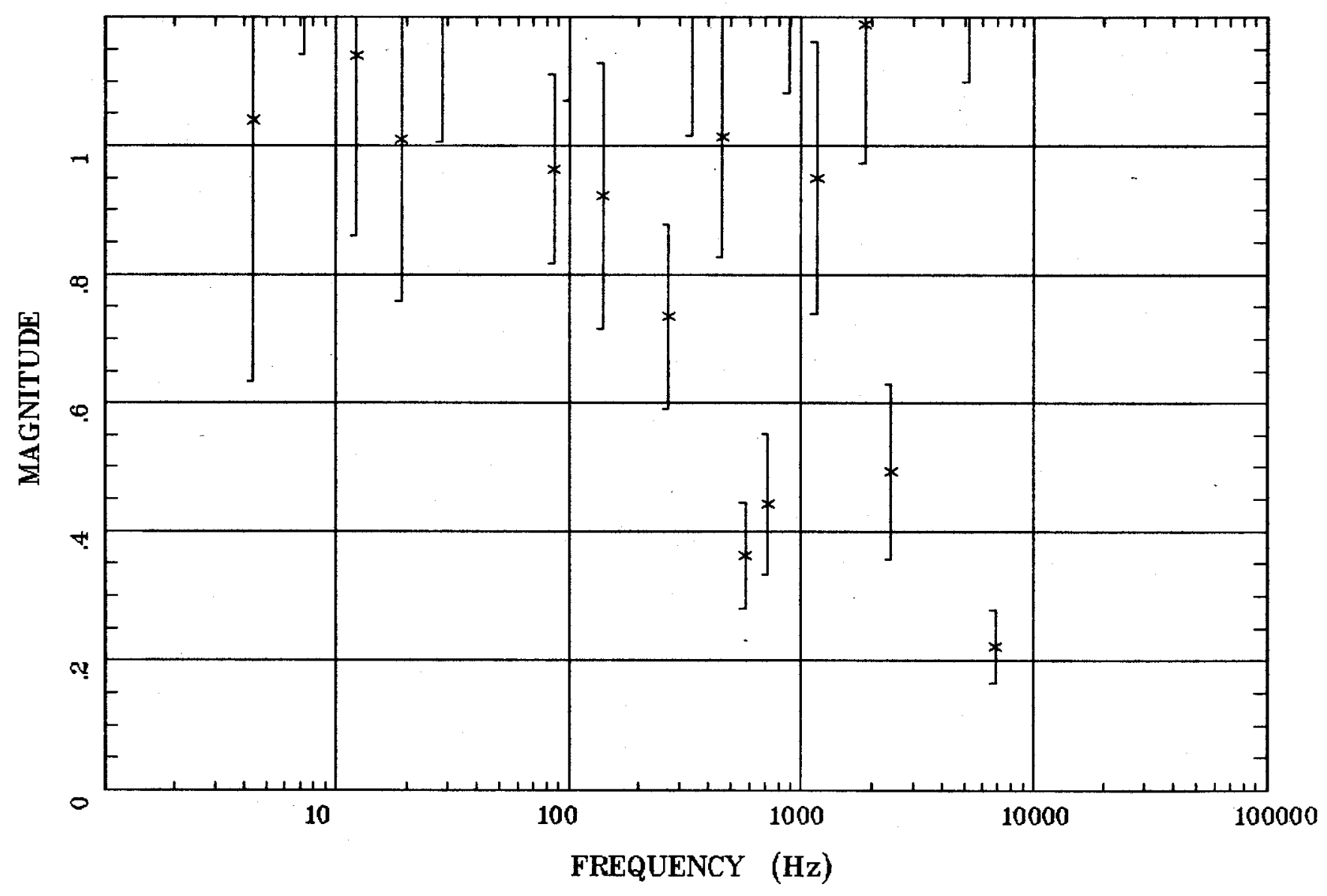

Client:

Remote: Lacal B

Acquired: 14:1 Aug 23, 1997

Survey Co:USGS GD-MRP Denver
Rotation:

Filename: al05.ave

Channels: Ch1 Ch2 Ch3 Ch4 Ch5 Ch8 Ch9

Plotted: 15:29 Mar 08, 2001

$<$ EMI - ElectroMagnetic Instruments 


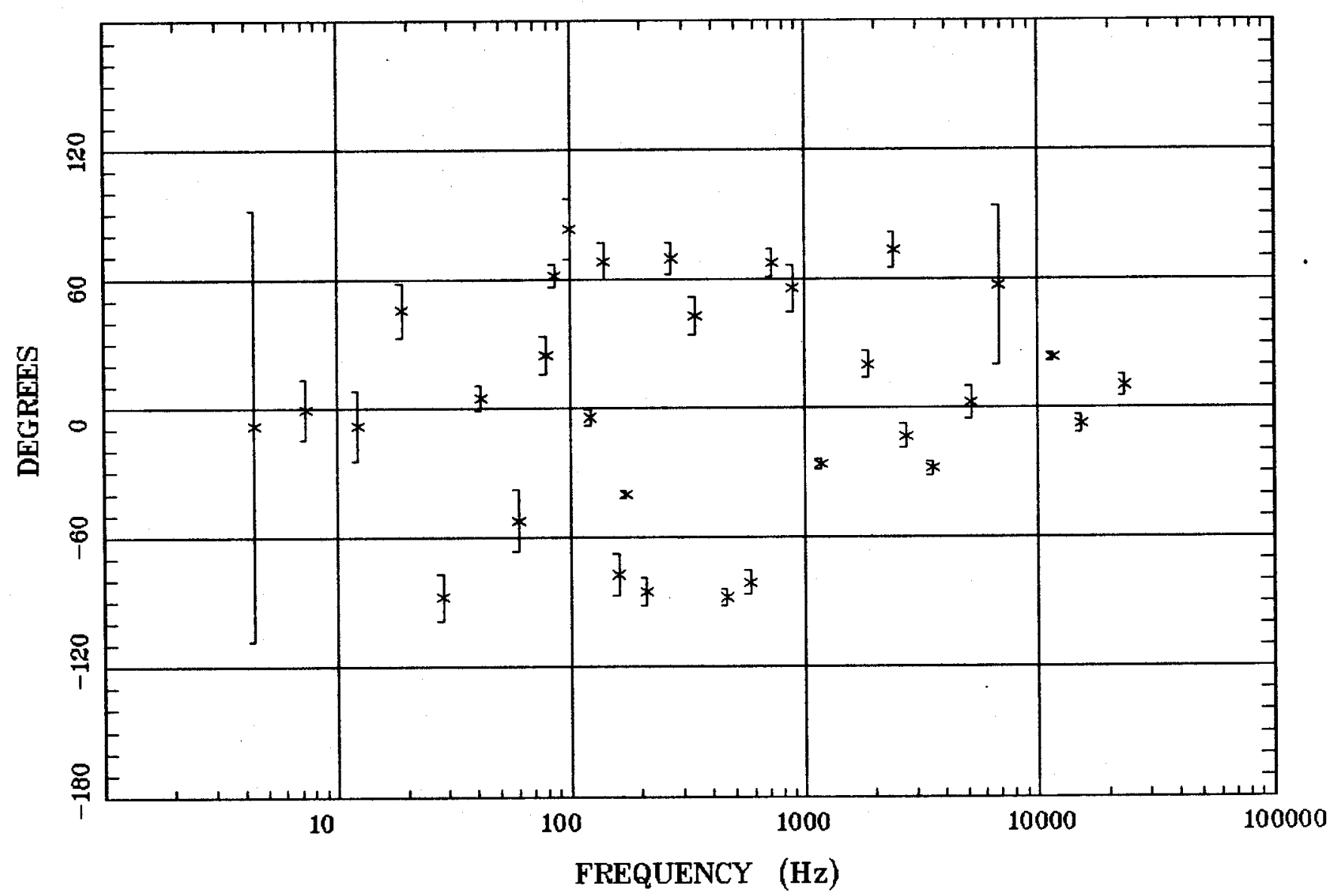

Client:

Remote: Local B Acquired: 14:1 Aug 23, 1997 Survey Co:USGS GD-MRP Denver
Rotation:

Filename: al05.ave

Channels: Ch1 Ch2 Ch3 Ch4 Ch5 Ch8 Ch9 Plotted: 15:29 Mar 08, 2001

< EMI - ElectroMagnetic Instruments > 
HzHx.x Coh HzHy.O

NM, Albuquerque, Volcanoes

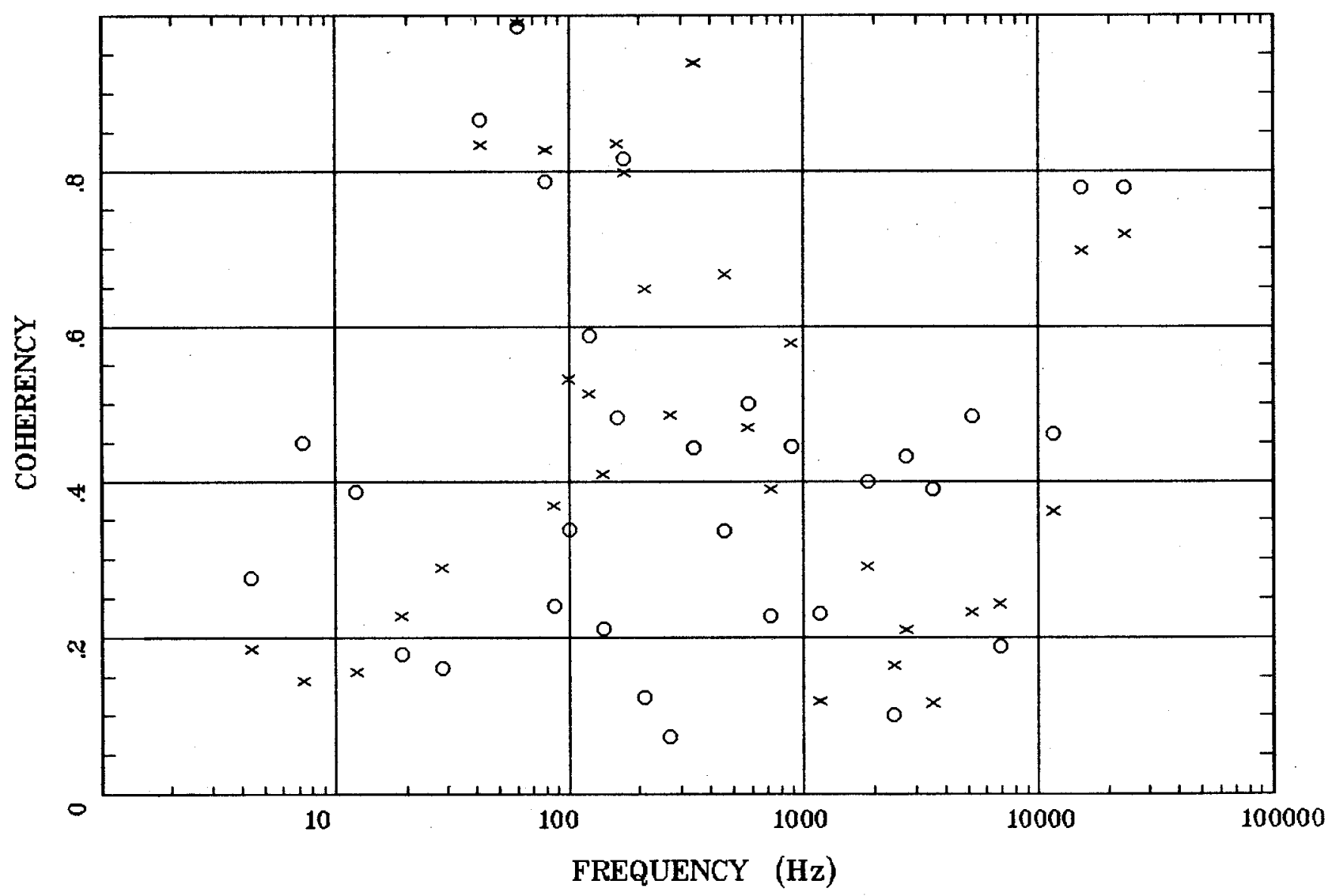

Client:

Remote: Local B

Acquired: 14:1 Aug 23, 1997

Survey Co:USGS GD-MRP Denver
Rotation:

Filename: al05.ave

Channels: Ch1 Ch2 Ch3 Ch4 Ch5 Ch8 Ch9

Plotted: 15:29 Mar 08, 2001

< EMI - ElectroMagnetic Instruments > 


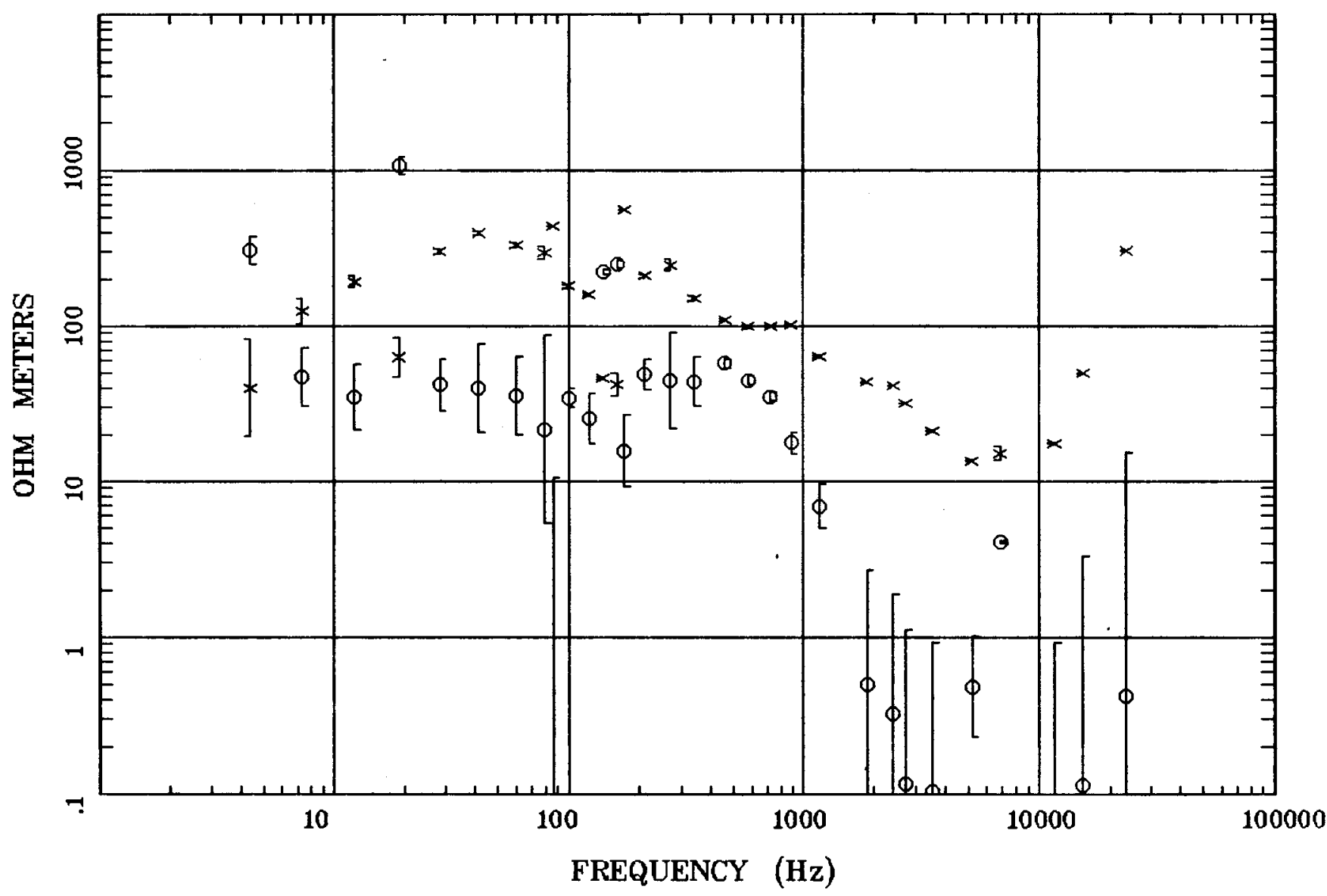

Client:

Remote: Local $B$

Acquired: 10:3 May 24, 1997

Survey Co:USGS GD-MRP Denver

Rotation:

Filename: rro7a1.all

Channels: Ch1 Ch2 Ch3 Ch4 Ch5 Ch8 Ch9

Plotted: 15:19 Mar 08, 2001

< EMI - ElectroMagnetic Instruments > 
Station 7

IMPEDANCE PHASE

Rio Rancho - Albuquerque, NM

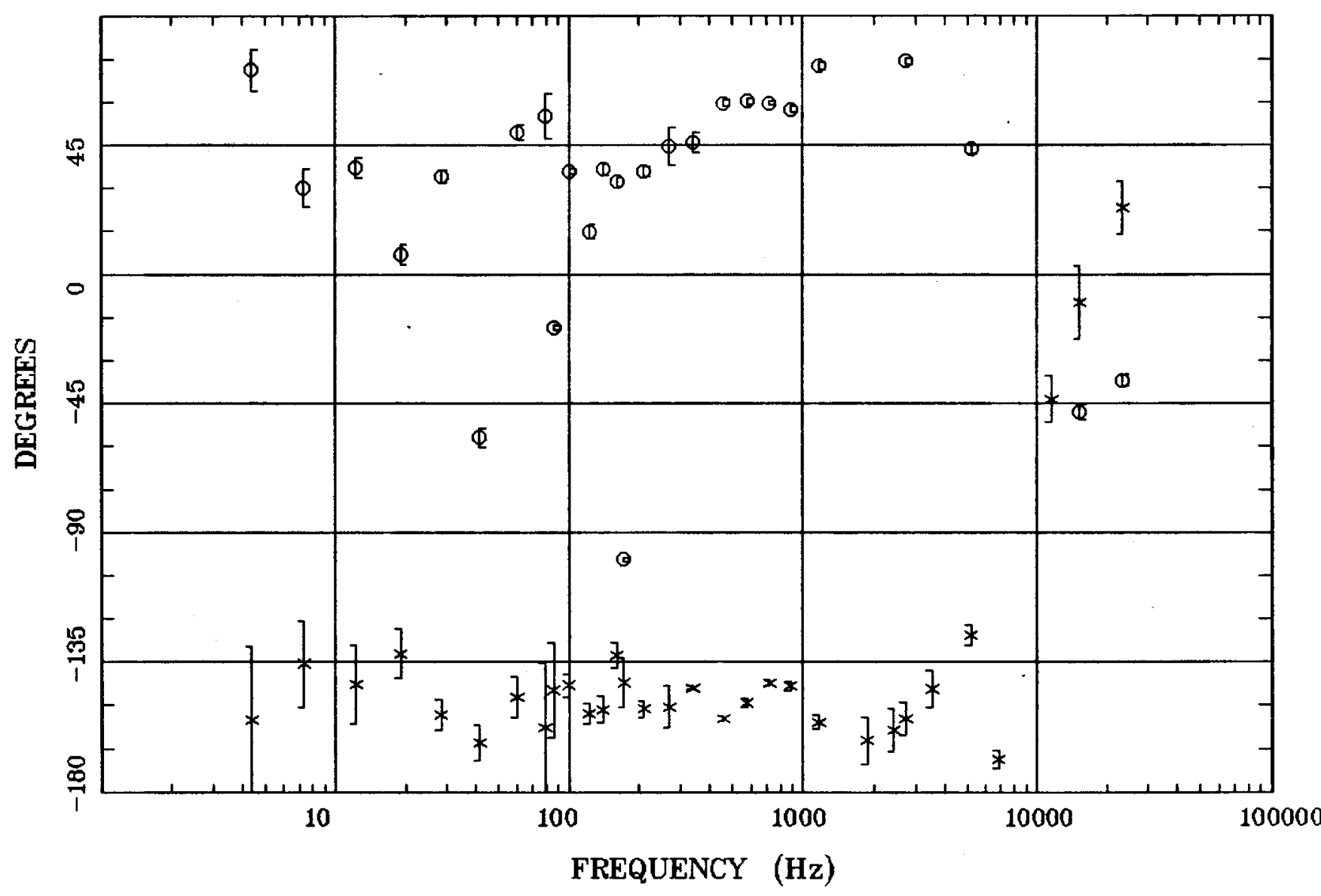

Client:

Remote: Local B

Acquired: 10:3 May 24, 1997 Survey Co:USGS GD-MRP Denver
Rotation:

Filename: rro7a1.all

Channels: Ch1 Ch2 Ch3 Ch4 Ch5 Ch8 Ch9

Plotted: 15:19 Mar 08, 2001

$<$ EMI - ElectroMagnetic Instruments 


\section{Station 7}

ROTATION ANGLE

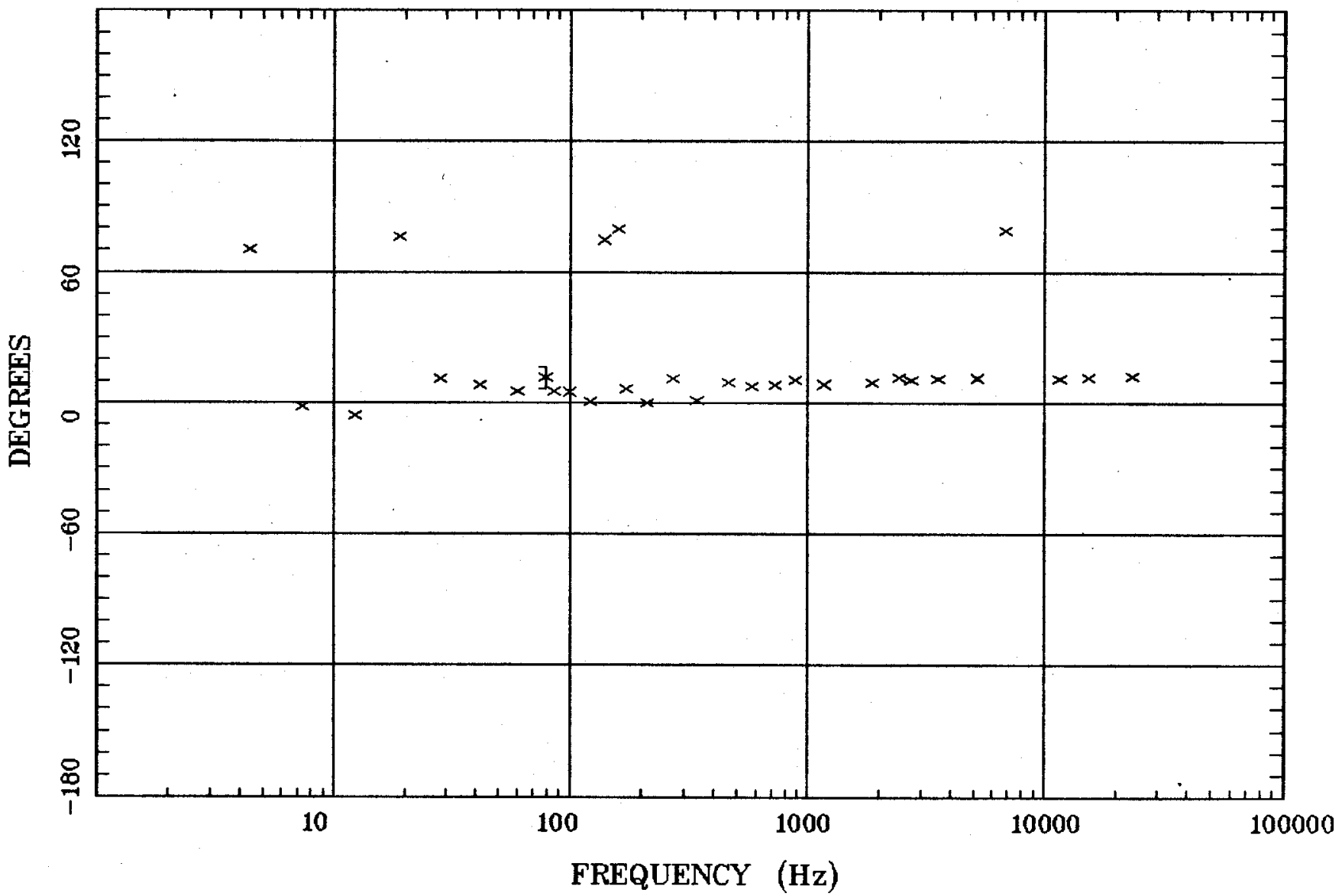

Client:

Remote: Local B

Acquired: $\quad 10: 3$ May 24, 1997

Survey Co:USGS GD-MRP Denver
Rotation:

Filename: rro7a1.all

Channels: Ch1 Ch2 Ch3 Ch4 Ch5 Ch8 Ch9

Plotted: 15:19 Mar 08, 2001

< EMI - ElectroMagnetic Instruments > 


\section{Station 7}

IMPEDANCE SKEW

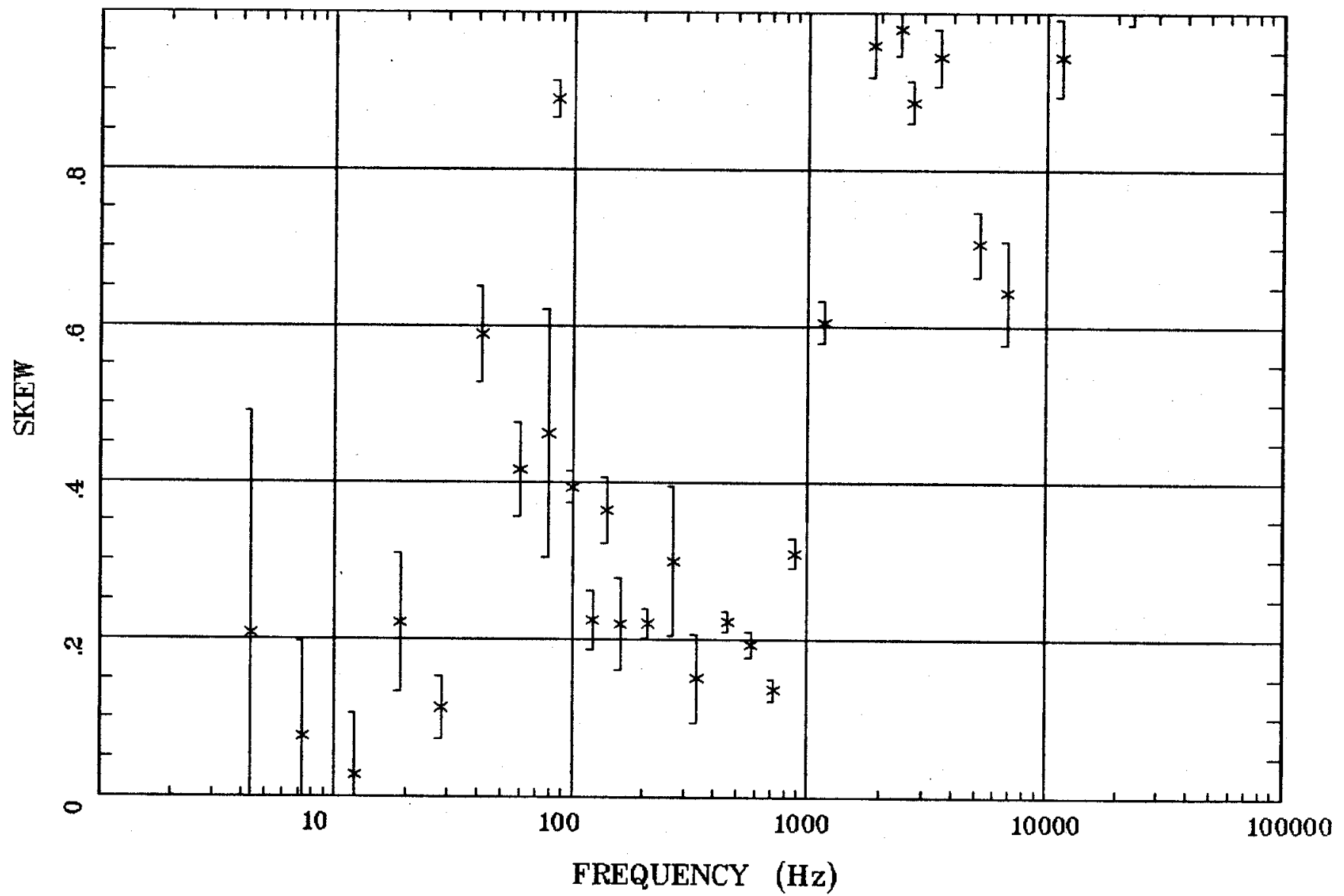

Rio Rancho - Albuquerque, NM

Rotation:

Filename: rro7a1.all

Channels: Ch1 Ch2 Ch3 Ch4 Ch5 Ch8 Ch9

Plotted: 15:19 Mar 08, 2001

< EMI - ElectroMagnetic Instruments >
Client:

Remote: Local B Acquired: 10:3 May 24, 1997 Survey Co:USGS GD-MRP Denver 
Station 7

E MULT Coh.

Rio Rancho - Albuquerque, NM

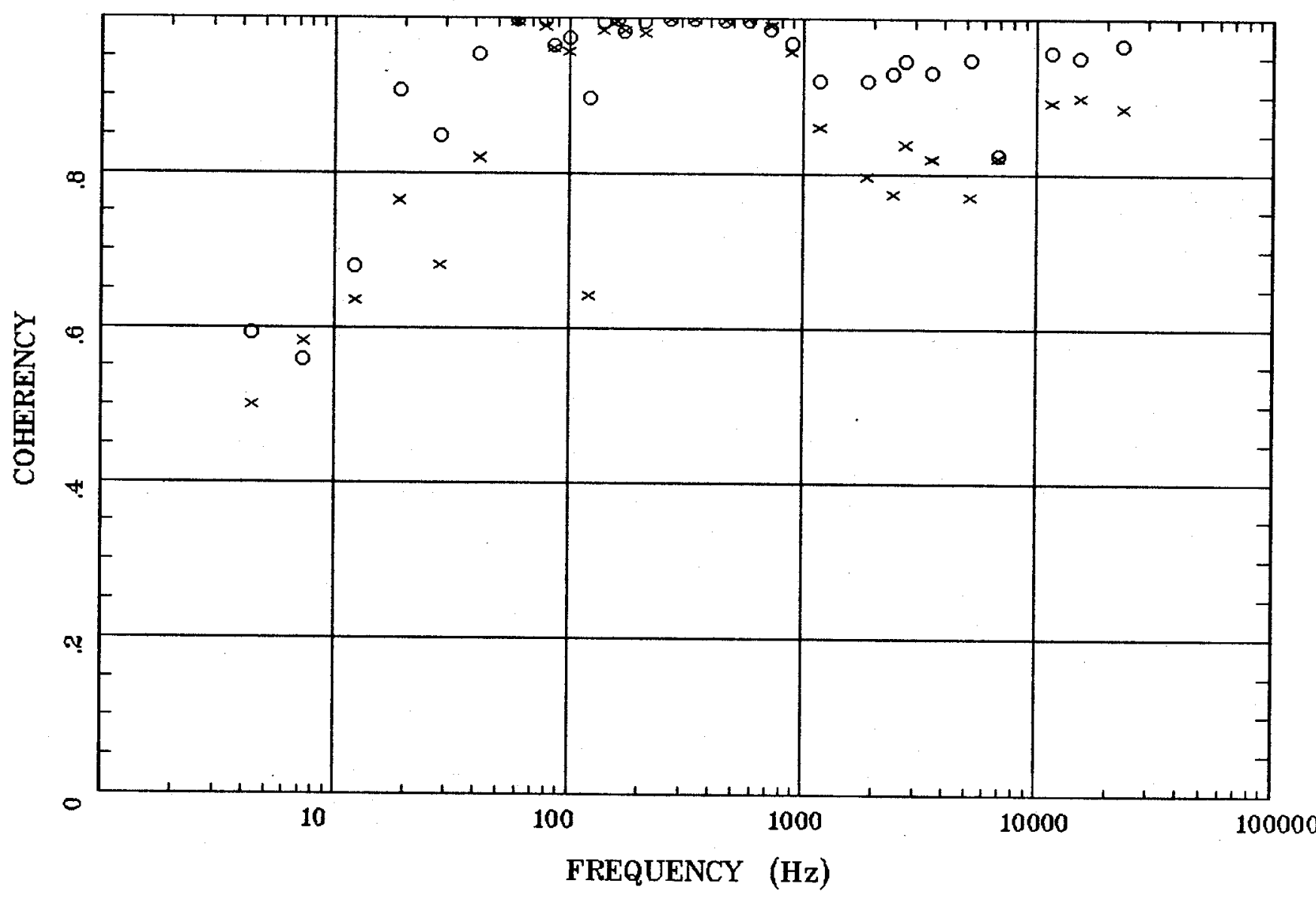

Client:

Remote: Local $B$

Acquired: 10:3 May 24, 1997

Survey Co:USGS GD-MRP Denver
Rotation:

Filename: rra7a1.all

Channels: Ch1 Ch2 Ch3 Ch4 Ch5 Ch8 Ch9 Plotted: 15:19 Mer 08, 2001

$<$ EMI - ElectroMagnetic Instruments 


\section{Station 7}

POLAR PLOTS

Rio Rancho - Albuquerque, NM

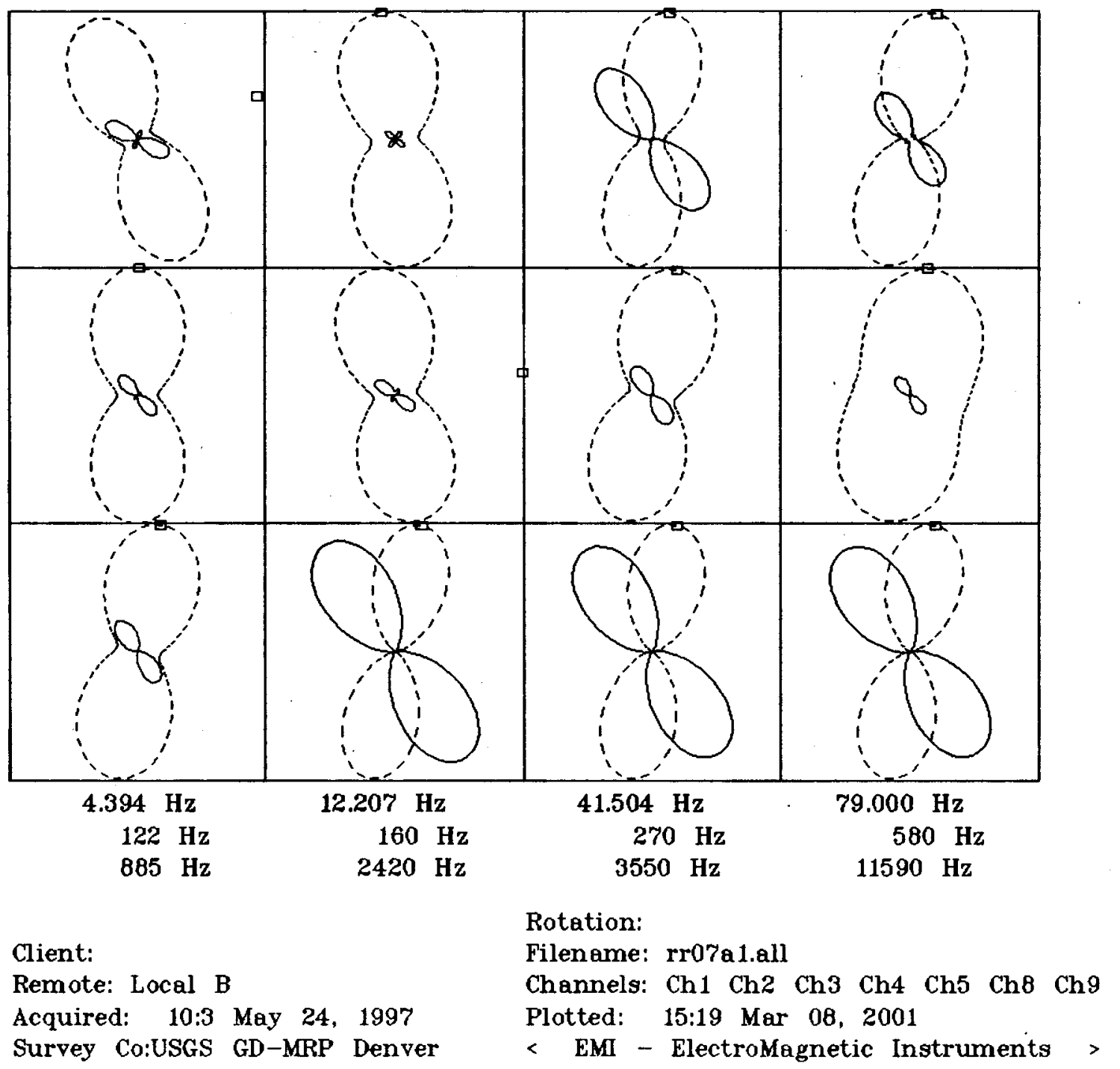


Station 7

TIPPER MAGNITUDE

Rio Rancho - Albuquerque, NM

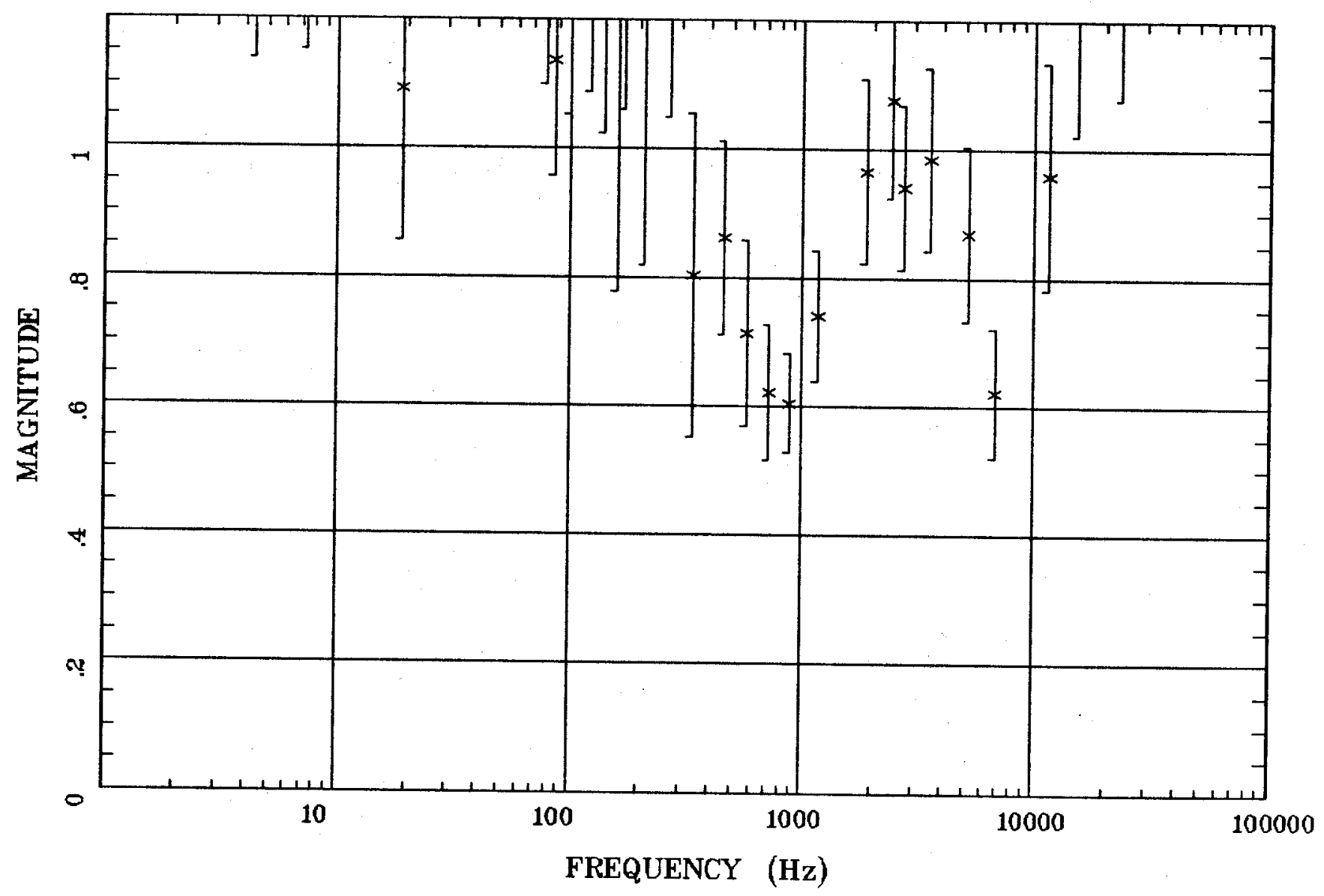

Client:

Remote: Local B

Acquired: 10:3 May 24, 1997

Survey Co:USGS GD-MRP Denver

Rotation:

Filename: rro7a1.all

Channels: Ch1 Ch2 Ch3 Ch4 Ch5 Ch8 Ch9

Plotted: 15:19 Mar 08, 2001

< EMI - ElectroMagnetic Instruments 
Station 7

TIPPER STRIKE

Rio Rancho - Albuquerque, NM

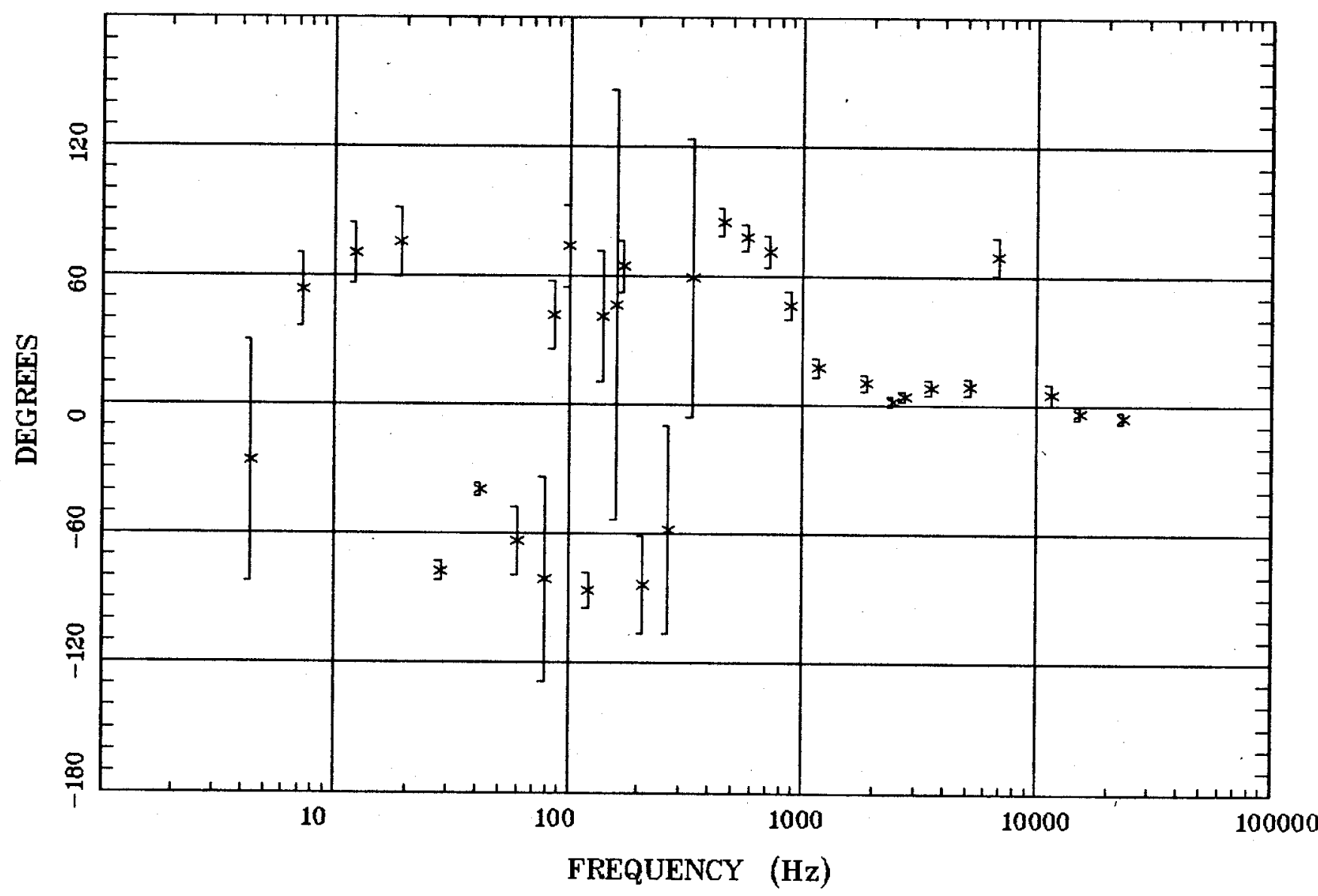

Client:

Remote: Local $B$

Acquired: 10:3 May 24, 1997

Survey Co:USGS GD-MRP Denver
Rotation:

Filename: rr07a1.all

Channels: Ch1 Ch2 Ch3 Ch4 Ch5 Ch8 Ch9

Plotted: 15:19 Mar 08, 2001

< EMI - ElectroMagnetic Instruments > 


\section{Station 7}

HzHx.x Coh HzHy.o

Rio Rancho - Albuquerque, NM

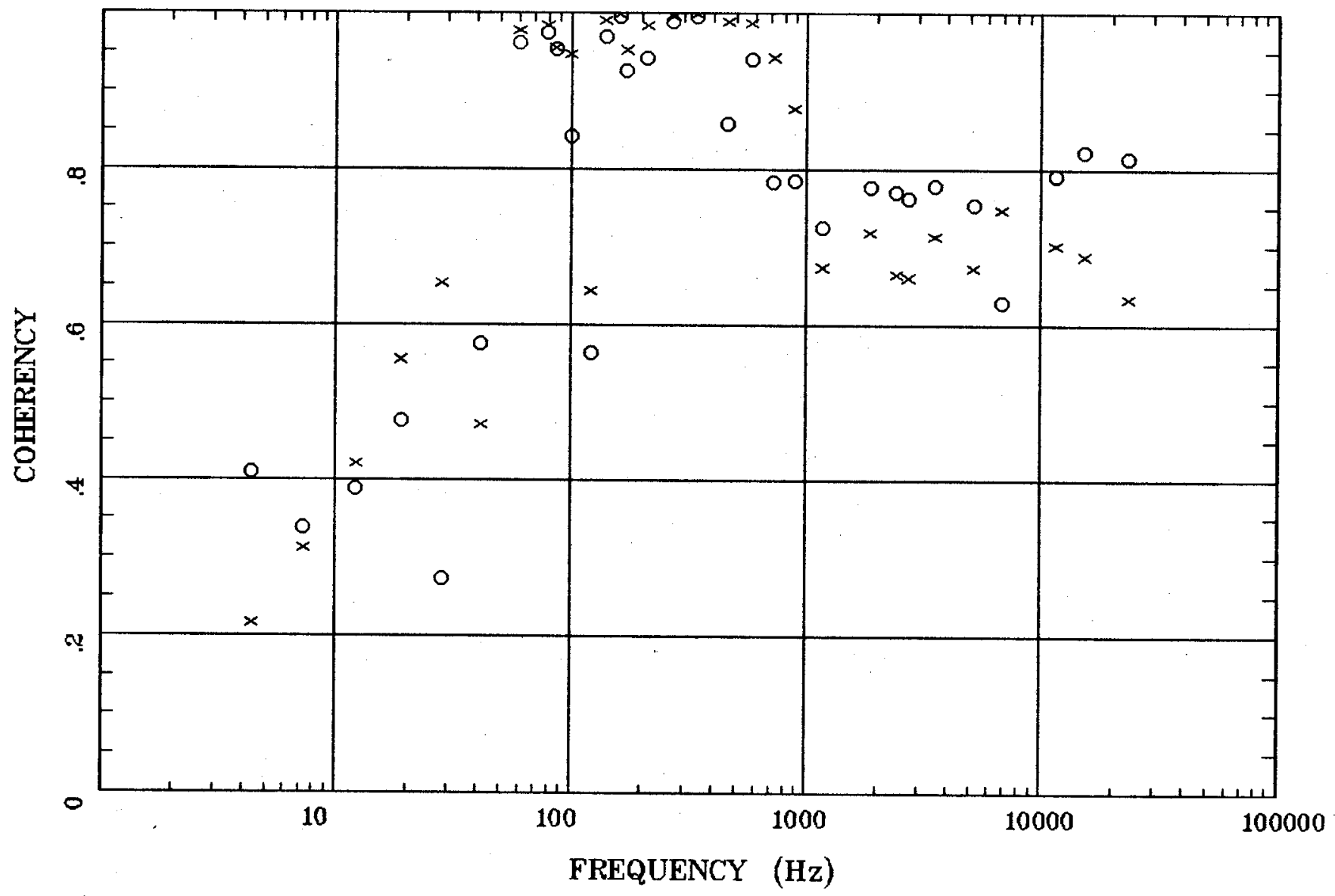

Client:

Remote: Local B

Acquired: 10:3 May 24, 1997

Survey Co:USGS GD-MRP Denver
Rotation:

Filename: rro7a1.all

Channels: Ch1 Ch2 Ch3 Ch4 Ch5 Ch8 Ch9

Plotted: 15:19 Mar 08, 2001

< EMI - ElectroMagnetic Instruments > 